\title{
Nuclear transport of the DNA fragmentation factor via the classical importin $\alpha / \beta$-pathway
}

\author{
Dissertation \\ zur Erlangung des Doktorgrades \\ der Mathematisch-Naturwissenschaftlichen Fakultäten \\ der Georg-August-Universität zu Göttingen
}

vorgelegt von

Sonja Neimanis

aus Hannover

Göttingen 2007 
D7

Referent:

Prof. Dr. D. Doenecke

Korreferent:

Prof. Dr. R. Hardeland

Tag der mündlichen Prüfung:

04.05.2007 
Die vorliegende Arbeit wurde in der Zeit von August 2003 bis März 2007 in der Abteilung Molekularbiologie unter Anleitung von Prof. Dr. D. Doenecke am Institut für Biochemie und Molekulare Zellbiologie der Georg-August-Universität zu Göttingen angefertigt.

I used the term "We" instead of "I" because during a $\mathrm{PhD}$ thesis one is usually supported by other persons, in particular the supervisors and lab members. However, the experiments described in this thesis and the writing were essentially done by myself. 


\section{Contents}

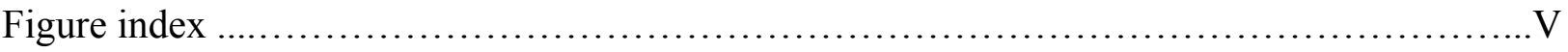

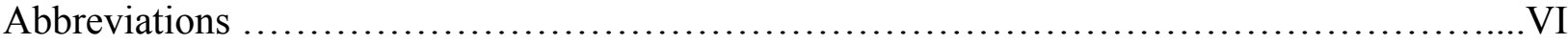

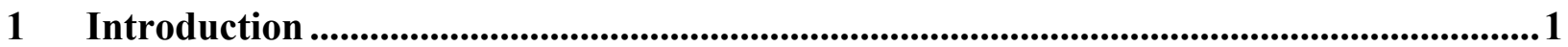

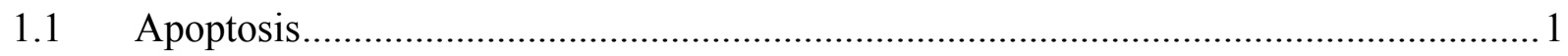

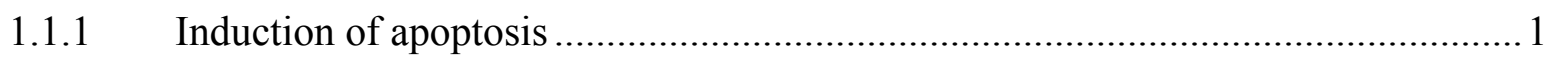

1.1.2 Caspases - central executioners of apoptosis ............................................... 3

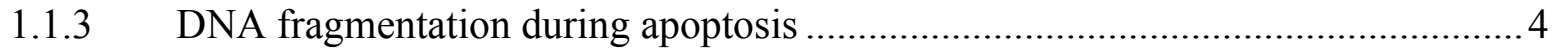

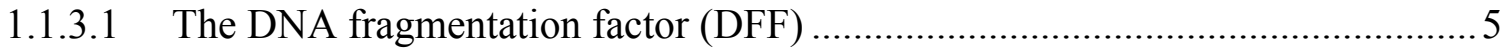

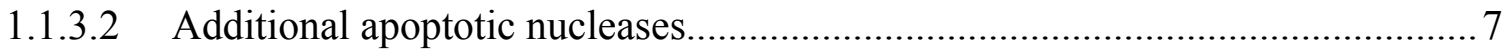

1.1.3.3 Functional relevance of apoptotic DNA fragmentation ................................... 8

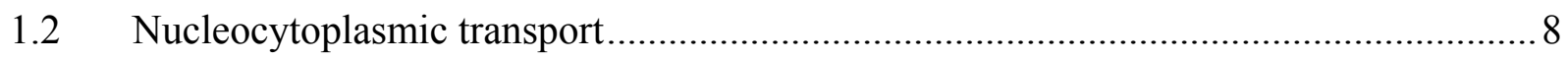

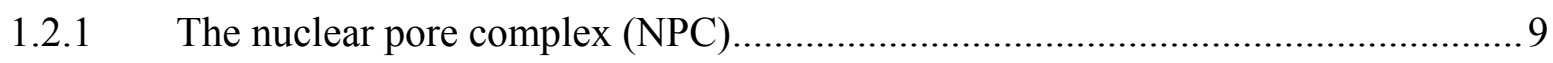

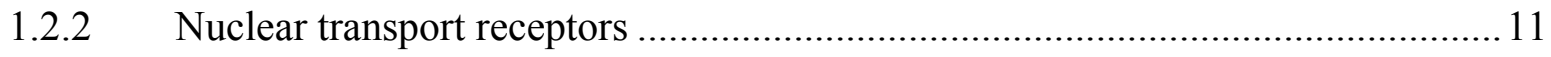

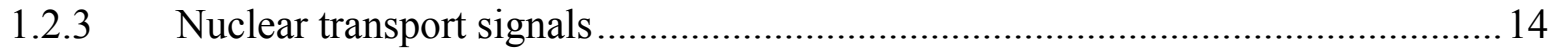

1.2.4 The small GTPase Ran and its function in nuclear transport .............................. 16

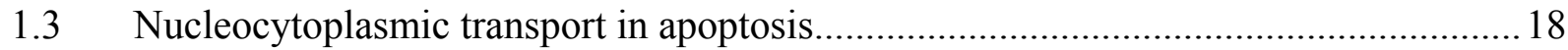

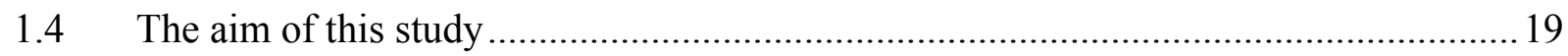

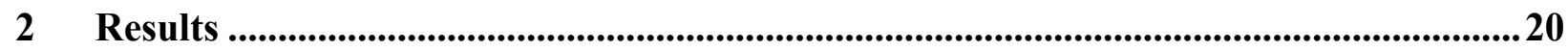

2.1 Nuclear import of the DFF complex is energy-dependent and requires access to

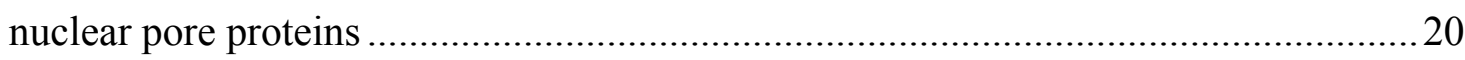

2.2 The importin $\alpha / \beta$ heterodimer specifically binds to the DFF complex ......................22

2.3 Specific binding of different importin $\alpha$ isoforms to the DFF complex .....................25

2.4 The importin $\alpha / \beta$ heterodimer is responsible for nuclear transport of the DFF complex

2.5 Nuclear import of the DFF complex requires the C-terminal regions of both subunits 28

2.6 Basic amino acids in both subunits are essential for nuclear import of DFF ............... 31

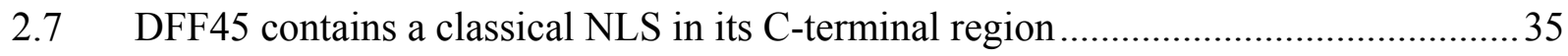

2.8 The C-terminal tail of DFF40 interacts with importins and also functions as NLS......38

2.9 Basic clusters in the C-terminal tails of DFF40 and DFF45 together presumably form a bipartite NLS 


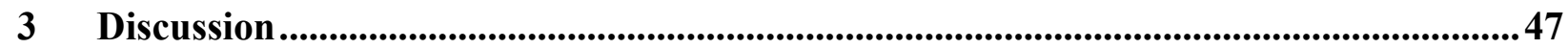

3.1 The DFF complex enters the nucleus via the classical importin $\alpha / \beta$-pathway............. 47

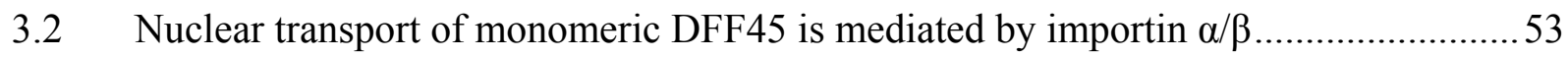

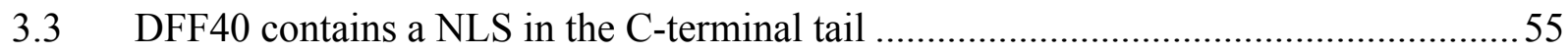

3.4 Binding models for the interaction between the DFF complex and importin $\alpha / \beta \ldots \ldots . .58$

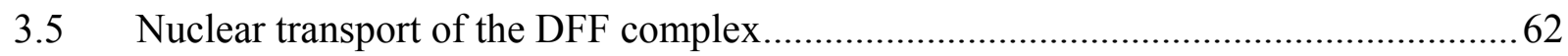

3.6 Activation of DFF40 in the nucleus requires nuclear entry of caspase-3 ...................6 64

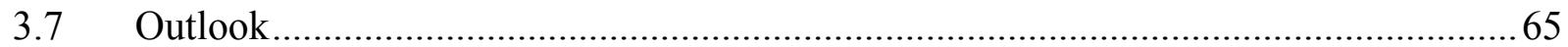

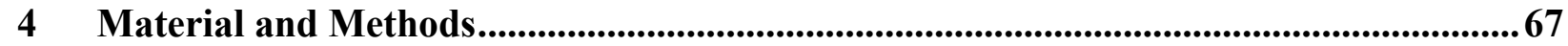

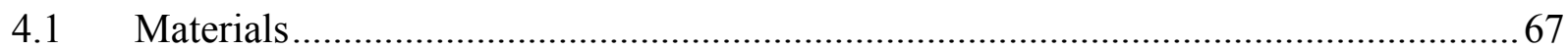

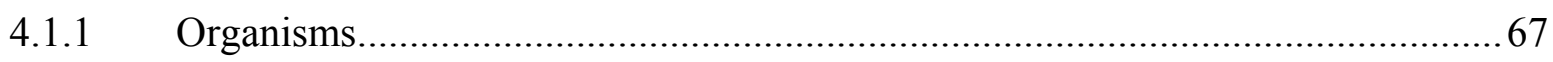

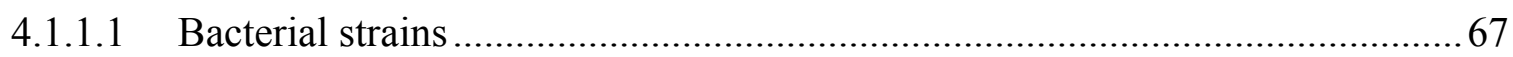

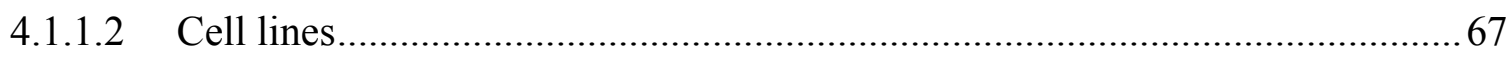

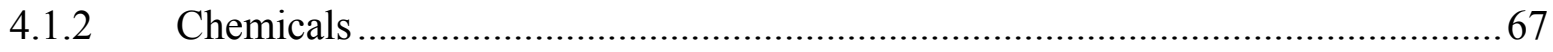

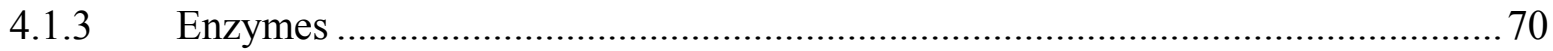

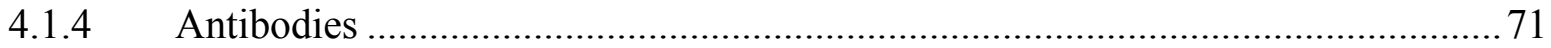

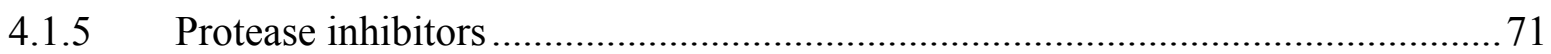

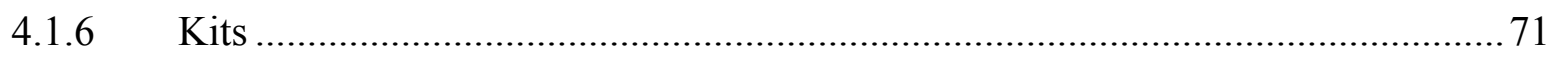

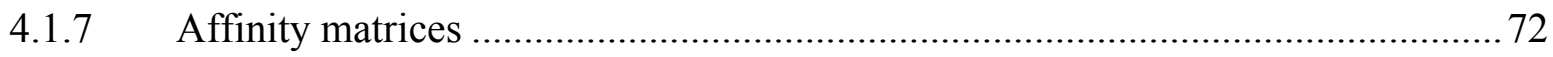

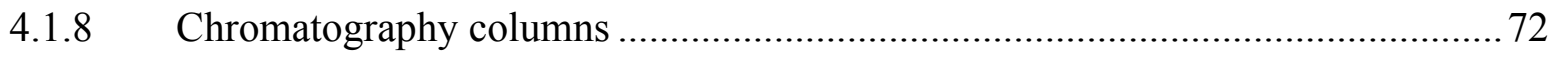

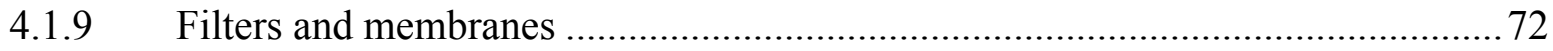

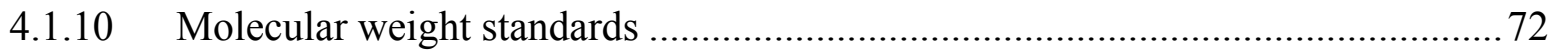

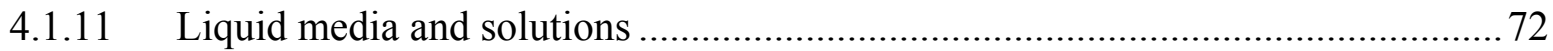

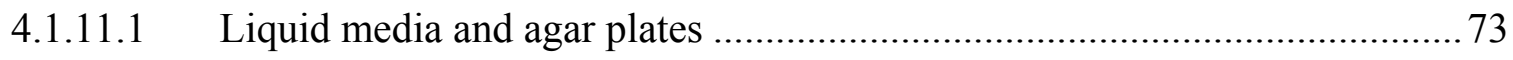

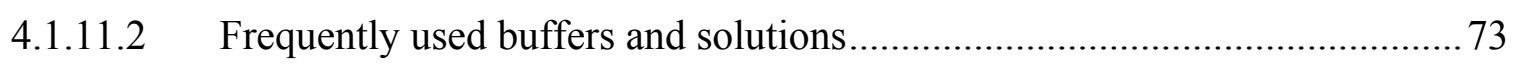

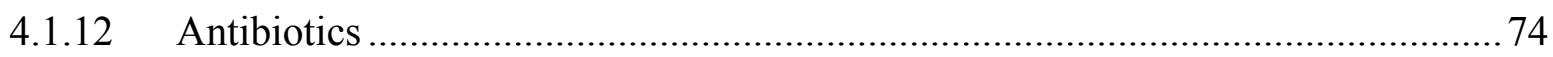

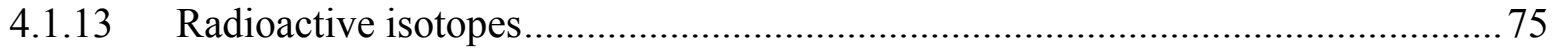

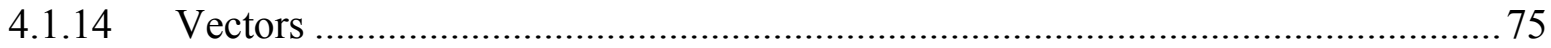

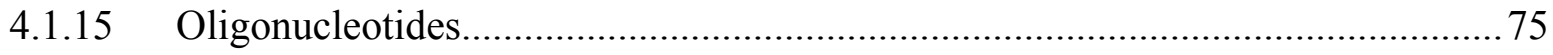

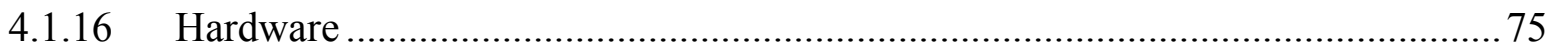

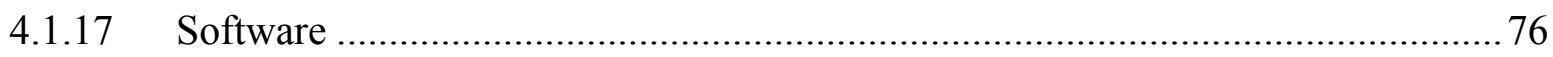

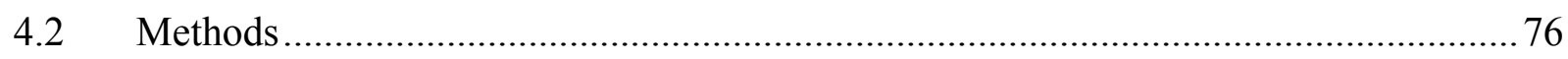

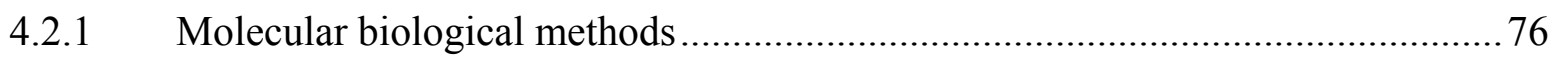

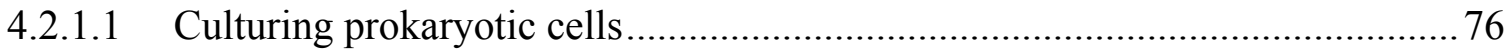




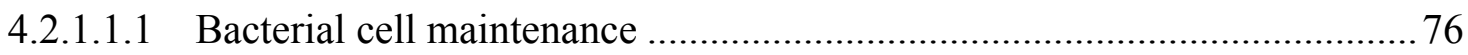

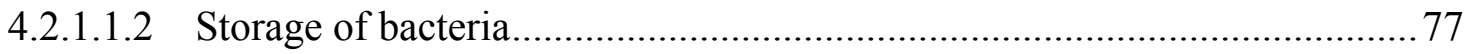

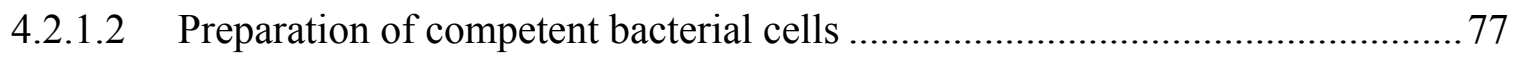

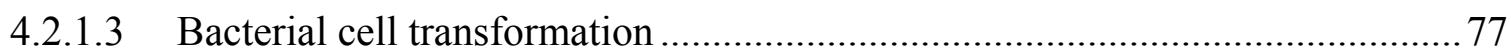

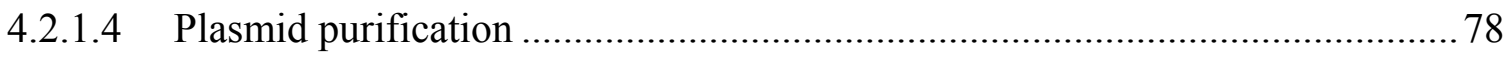

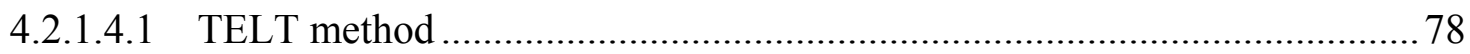

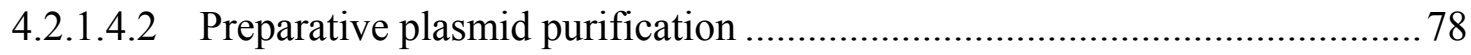

4.2.1.5 Concentration of DNA by ethanol/isopropanol precipitation .......................... 78

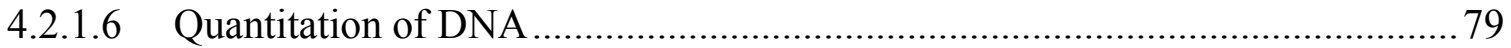

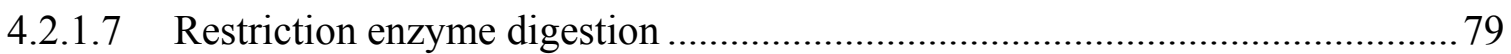

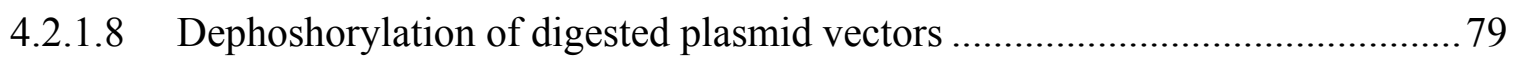

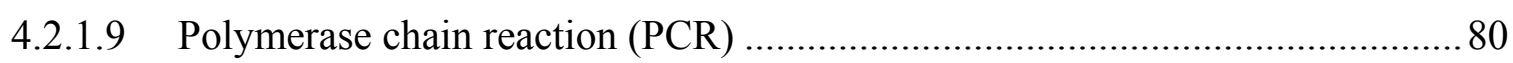

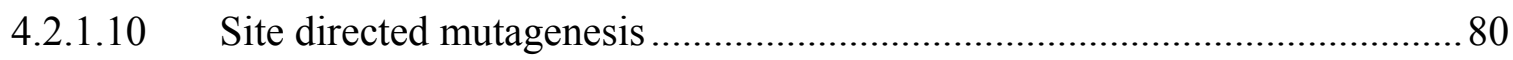

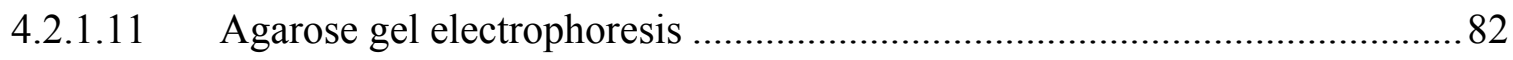

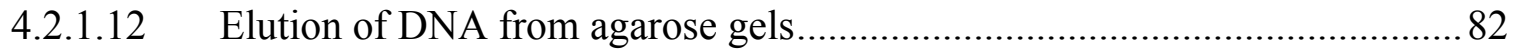

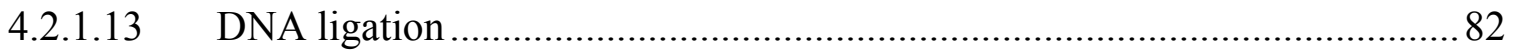

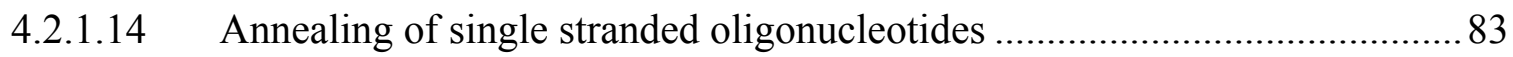

4.2.1.15 End-labeling of DNA with T4 polynucleotide kinase (T4 PNK).................. 83

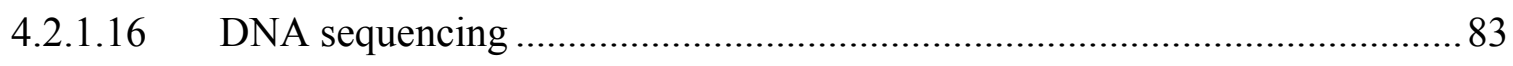

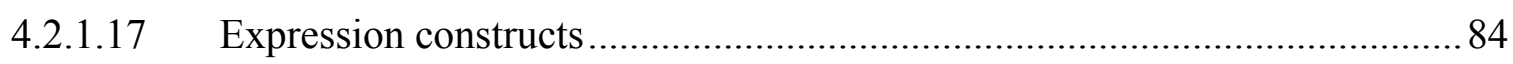

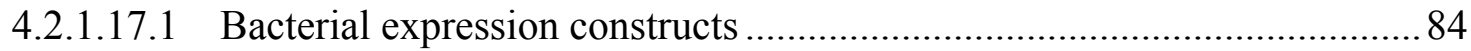

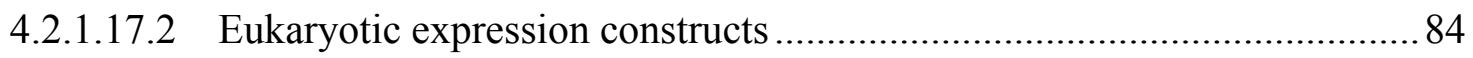

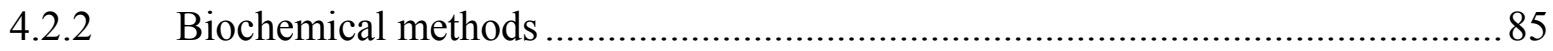

4.2.2.1 SDS-polyacrylamide gel electrophoresis (SDS-PAGE) ................................. 85

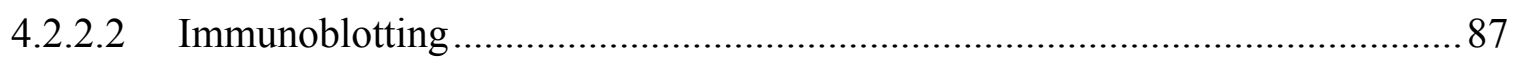

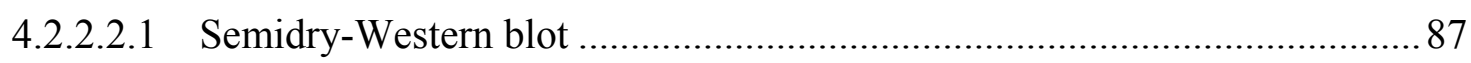

4.2.2.2.2 Detection of immobilized proteins using antibodies................................. 87

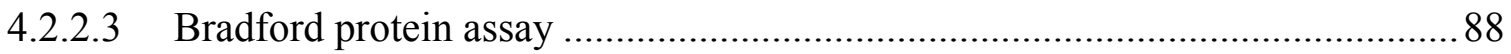

4.2.2.4 Recombinant protein expression and purification.......................................... 88

4.2.2.5 Expression and purification of nuclear transport cargoes ............................... 89

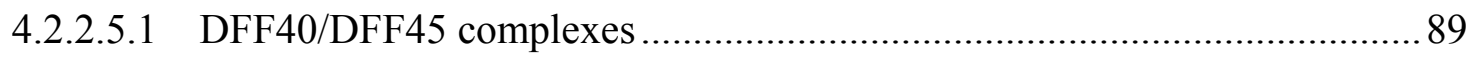

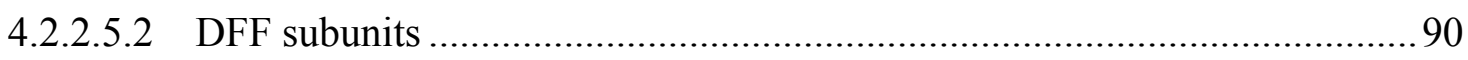

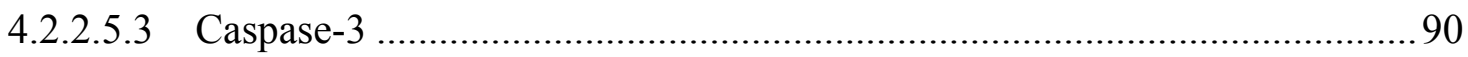

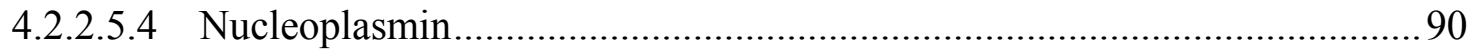


4.2.2.5.5 Expression and purification of import receptors and supplementary factors 90

4.2.2.5.5.1 Ran and NTF2 91

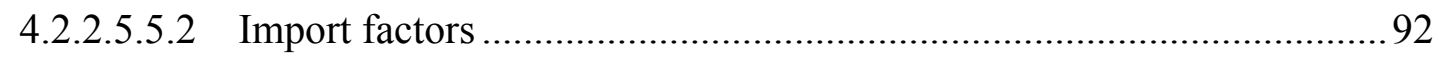

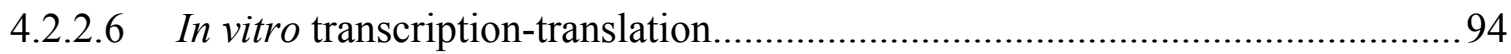

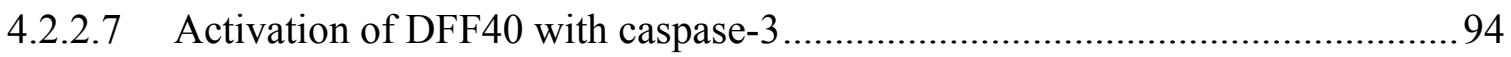

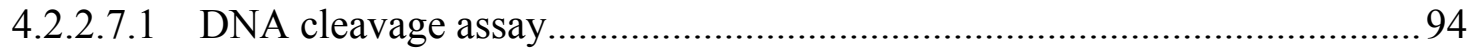

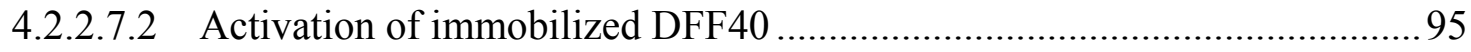

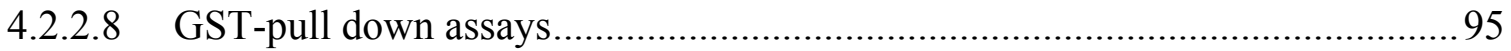

4.2.2.8.1 In vitro binding studies with bacterially expressed proteins ......................95

4.2.2.8.2 GST-pull down assays after overexpression of DFF subunits in HeLa

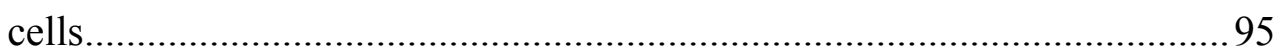

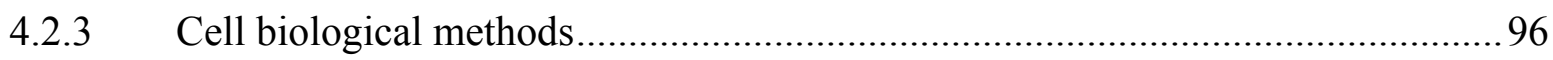

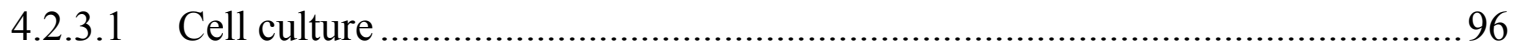

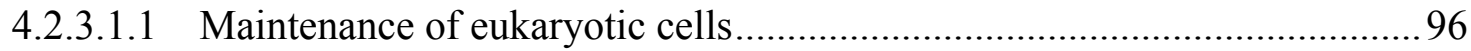

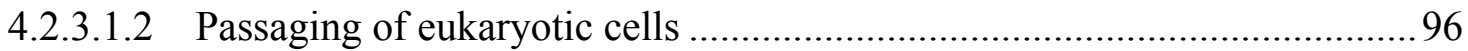

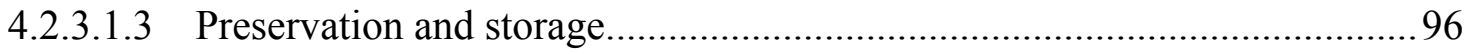

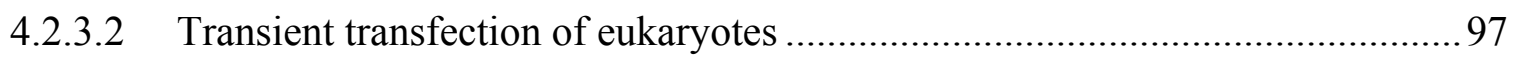

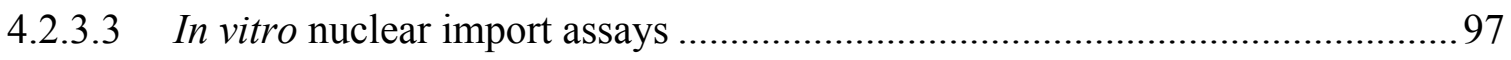

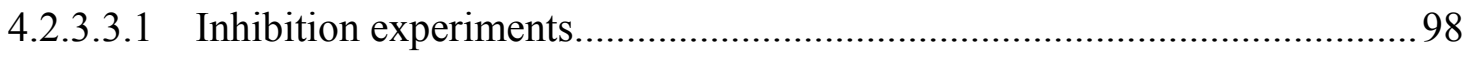

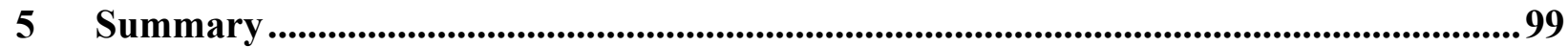

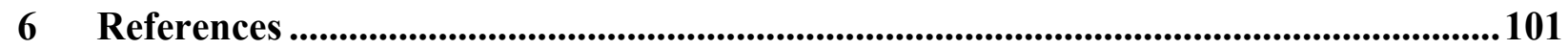

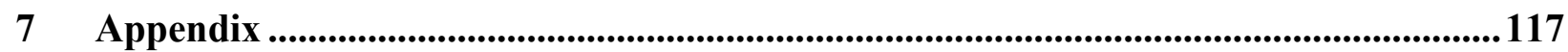




\section{Figure index}

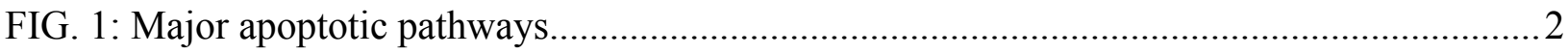

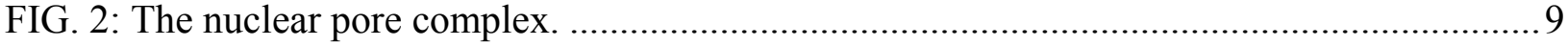

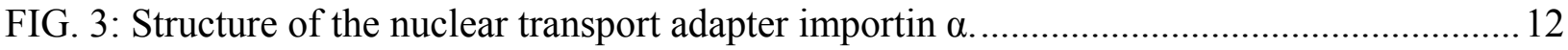

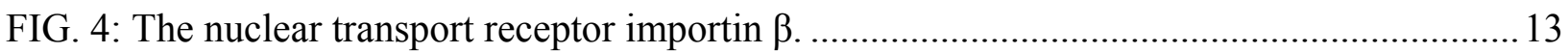

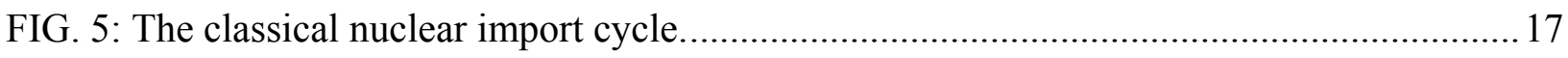

FIG. 6: The DFF complex is actively imported into the nucleus......................................... 21

FIG. 7: The DFF40/DFF45 complex specifically interacts with the importin $\alpha / \beta$ heterodimer...23

FIG. 8: Specific binding of different importin $\alpha$ isoforms to the DFF complex.........................26

FIG. 9: Nuclear transport of DFF is mediated by the importin $\alpha / \beta$ heterodimer. .......................2 27

FIG. 10: The C-terminal regions of DFF40 and DFF45 are required for nuclear import of the DFF complex.

FIG. 11: Basic amino acids in the C-terminal regions of DFF40 and DFF45 are essential for

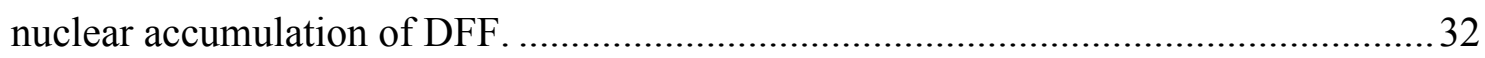

FIG. 12: Interaction of fluorescently tagged mutant DFF subunits. ....................................... 34

FIG. 13: The C-terminal tail of DFF45 exhibits a classical NLS that is necessary and sufficient

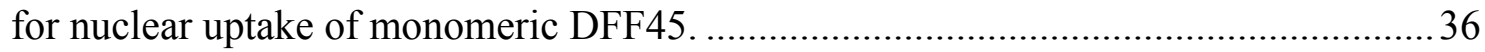

FIG. 14: The C-terminus of DFF45 is necessary for the interaction between the DFF complex and importin $\alpha / \beta$.

FIG. 15: The C-terminal tail of DFF40 contributes to the interaction between the DFF complex and importin $\alpha / \beta$.

FIG. 16: The C-terminal tail of DFF40 contains a functional NLS

FIG. 17: The amino-terminal region of importin $\beta$ does not participate in the binding of importin $\alpha / \beta$ to the DFF complex

FIG. 18: The minor binding site of importin $\alpha$ is required for efficient binding of importin $\alpha / \beta$ to the DFF complex

FIG. 19: Nuclear accumulation of the DFF complex does not require lysine 308 and 309 of DFF40

FIG. 20: Lysines 308 and 309 in DFF40 are not required for importin $\alpha / \beta$ binding

FIG. 21: Sequence alignment of DFF45.

FIG. 22: Sequence alignment of DFF40.

FIG. 23: Binding of importin $\alpha / \beta$ to a NLS unique for the DFF complex. 60

FIG. 24: Model for the nuclear import of human DFF complex and monomeric DFF45. 


\section{Abbreviations}

$\begin{array}{ll}\text { A } & \text { adenine } \\ \text { aa } & \text { amino acid } \\ \text { ad } & \text { add up to }\end{array}$

AIF apoptosis inducing factor

AKAP 95 A-Kinase-Anchoring Protein 95

amp ampicilline

Apaf Apoptotic protease activating factor

APS ammonium peroxodisulfate

ARM armadillo

ATP adenosine triphosphate

Bax Bcl-2 accociated $X$ protein

Bcl-2 protein isolated from a B cell lymphoma

Bid Bcl-2 interacting domain

BME $\quad \beta$-mercaptoethanol

bp base pairs

BSA bovine serum albumin

C cytosine

CAD caspase-activated DNase

CARD caspase recruitment domain

CIDE cell death-inducing DFF45-like effector

CIP Calf intestinal alkaline phosphatase

CPAN caspase-activated nuclease

CRM chromosome region maintenance

d distilled

Da Dalton

DAPI 4'-6-Diamino-2-phenylindol-dihydrochlorid

DED death effector domain

ddNTP dideoxy nucleoside triphosphate (ddATP, ddCTP, ddGTP, ddTTP)

DFF35 DNA fragmentation factor, $35 \mathrm{kDa}$ subunit

DFF40 DNA fragmentation factor, $40 \mathrm{kDa}$ subunit

DFF45 DNA fragmentation factor, $45 \mathrm{kDa}$ subunit

DISC death-inducing signalling complex 


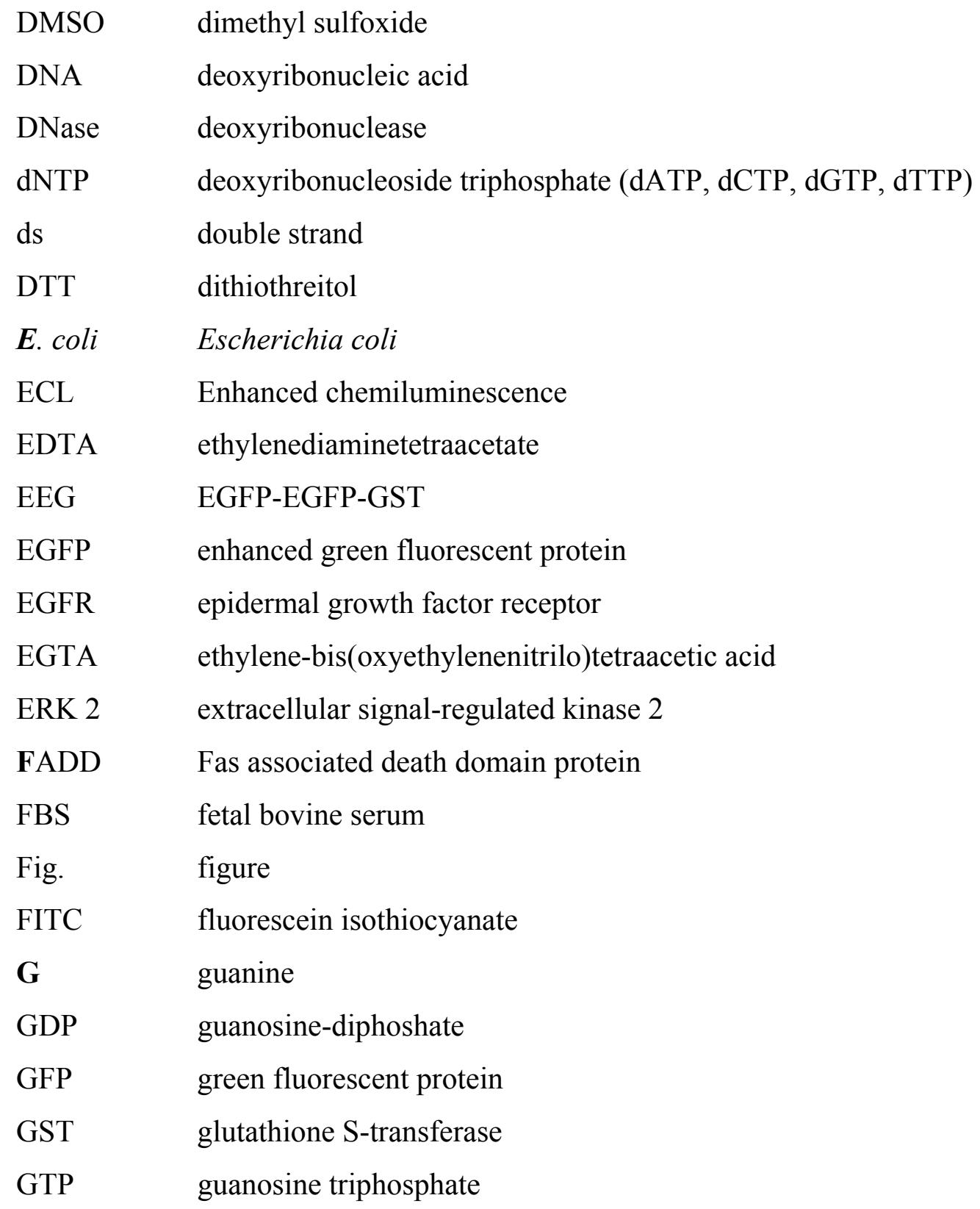

HA hemagglutinin

HEAT (Huntingtin, Elongation factor 3, "A" subunit of protein phosphatase A, and TOR1 lipid kinase)

HEPES $\quad N$-2-hydroxyethylpiperazine- $N$ '-2-ethanesulfonic acid

HIF hypoxia inducible factor

HIV human immunodeficiency virus

HMG high mobility group

HMW high molecular weight

hnRNP heterogenous nuclear ribonucleoprotein

HRP horseradish peroxidase

IBB importin $\beta$ binding 


\begin{tabular}{|c|c|}
\hline ICAD & inhibitor of CAD \\
\hline ICAD-S & short form of ICAD \\
\hline IgG & immunoglobulin $\mathrm{G}$ \\
\hline $\operatorname{imp}$ & importin \\
\hline IPTG & isopropylthio- $\beta$-D-galactoside \\
\hline $\mathbf{k b p}$ & kilobase pairs \\
\hline $\mathrm{kDa}$ & kilodalton \\
\hline $\mathbf{L B}$ & Luria Bertani \\
\hline MCS & multiple cloning site \\
\hline mRNA & messenger RNA \\
\hline $\mathrm{mt}$ & myc-tag \\
\hline MW & molecular weight \\
\hline $\mathbf{N E}$ & nuclear envelope \\
\hline NES & nuclear export signal \\
\hline NLS & nuclear localization signal \\
\hline NPC & nuclear pore complex \\
\hline NPI-1 & nucleoprotein interactor 1 \\
\hline NTF2 & nuclear transport factor 2 \\
\hline OD & optical density \\
\hline $\mathbf{p}$ & plasmid \\
\hline p.a. & pro analysi \\
\hline PAGE & polyacrylamide gelelectrophoresis \\
\hline PBS & phosphate buffered saline \\
\hline PCR & polymerase chain reaction \\
\hline PLSCR1 & Phospholipid scramblase 1 \\
\hline PTHrP & parathyroid hormone-related protein \\
\hline Ran & Ras related nuclear protein \\
\hline RanBP & Ran binding protein \\
\hline RanGAP & Ran GTPase activating protein \\
\hline RanGEF & Ran guanine nucleotide exchange factor \\
\hline $\mathrm{RCC} 1$ & regulator of chromosome condensation 1 \\
\hline Rch1 & RAG cohort protein 1 \\
\hline RFP & red fluorescent protein \\
\hline retic & reticulocyte lysate \\
\hline
\end{tabular}




\begin{tabular}{|c|c|}
\hline RNA & ribonucleic acid \\
\hline RNase & ribonuclease \\
\hline RNP & ribonucleoprotein \\
\hline rpm & rounds per minute \\
\hline rRNA & ribosomal RNA \\
\hline SCD & Synthetic complete medium with glucose \\
\hline SCGL & Synthetic complete medium with glycerole and lactic acid \\
\hline S. cerevisiae & Saccharomyces cerevisiae \\
\hline SDS & sodium dodecyl sulfate \\
\hline ss & single strand \\
\hline STAT & signal transducer and activator of transcription \\
\hline SUMO & small ubiquitin-related modifier/modification \\
\hline SV40 & simian virus 40 \\
\hline $\mathbf{T}$ & tymine \\
\hline TBE & Tris-boric acid-EDTA \\
\hline TBS & Tris buffered saline \\
\hline TBST & Tris buffered saline containin Tween 20 \\
\hline $\mathrm{TE}$ & Tris-EDTA buffer \\
\hline TELT & Tris-EDTA-LiCl-Triton \\
\hline TEMED & tetramethylethylenediamine \\
\hline TNF-R1 & tumor necrosis factor receptor type 1 \\
\hline TRIS & tris(hydroxymethyl)aminomethane \\
\hline $\operatorname{trn}$ & transportin \\
\hline UV & ultraviolet \\
\hline WGA & wheat germ agglutinin \\
\hline wt & wild type \\
\hline YEPD & yeast-extract-peptone-glucose medium \\
\hline YPGLA & yeast-extract-peptone-glucose-lactic acid medium \\
\hline
\end{tabular}


$\underline{\text { One letter code for amino acids: }}$

$\begin{array}{ll}\text { A } & \text { alanine } \\ \text { C } & \text { cysteine } \\ \text { D } & \text { aspartic acid } \\ \text { E } & \text { glutamic acid } \\ \text { F } & \text { phenylalanine } \\ \text { G } & \text { glycine } \\ \text { H } & \text { histidine } \\ \text { I } & \text { isoleucine } \\ \mathrm{K} & \text { lysine } \\ \text { L } & \text { leucine } \\ \mathrm{M} & \text { methionine } \\ \mathrm{N} & \text { asparagine } \\ \mathrm{P} & \text { proline } \\ \mathrm{Q} & \text { glutamine } \\ \mathrm{R} & \text { arginine } \\ \mathrm{S} & \text { serine } \\ \mathrm{T} & \text { threonine } \\ \mathrm{V} & \text { valine } \\ \mathrm{W} & \text { tryptophan } \\ \mathrm{Y} & \text { tyrosine } \\ \mathrm{X} & \text { any amino acid }\end{array}$




\section{Introduction}

\subsection{Apoptosis}

For the development and life of multicellular organisms the controlled removal of cells that are in excess or abnormal is indispensable. Programmed cell death called apoptosis is the evolutionarily conserved process that enables organisms to eliminate cells in a highly regulated manner. Apoptosis plays an important role in tissue homeostasis, development, immune response and defense against pathogens (Jacobson et al., 1997; Rathmell and Thompson, 2002). Dysregulation of this process causes a number of human diseases such as cancer, neurodegenerative disorders and autoimmune diseases (Green and Evan, 2002; Hanahan and Weinberg, 2000; Thompson, 1995; Vaux and Flavell, 2000).

The controlled removal of a cell is accompanied by various morphological changes including cell shrinkage, membrane blebbing, chromatin condensation and nuclear breakdown (reviewed in Hacker, 2000). Finally, the cell disintegrates into apoptotic bodies which are engulfed by macrophages and surrounding cells (reviewed in Savill and Fadok, 2000). These morphological changes were the only indications for cell death in the first studies on apoptosis (Kerr et al., 1972) and are still the basis for its detection today.

Be aware, that the processes described below represent just an overview of apoptosis. The mechanisms involved in apoptosis induction and signal transduction are much more complex and highly regulated, e.g. in addition to activation processes certain safe-guarding mechanisms exist which inhibit apoptosis in healthy cells.

\subsubsection{Induction of apoptosis}

In mammalian cells apoptosis can be induced by two different pathways (Fig. 1), the extrinsic (death-receptor) pathway and the intrinsic (mitochondrial) pathway (reviewed in Boatright and Salvesen, 2003; Fuentes-Prior and Salvesen, 2004). The extrinsic pathway is responsible for apoptosis in development and immune response and is triggered by transmembrane deathreceptors of the TNF-R1 (tumor necrosis factor receptor type 1) superfamily such as CD95 (Apo-1/Fas) (Ashkenazi and Dixit, 1998). Ligand binding to CD95 leads to receptor clustering and the formation of a death-inducing signaling complex (DISC). The adapter protein FADD (Fas associated death domain protein) interacts with the cytoplasmic receptor domain and recruits other proteins (e.g. procaspases, see below) to the DISC (Algeciras-Schimnich et al., 2002). In this way, the apoptotic signal is transduced. 


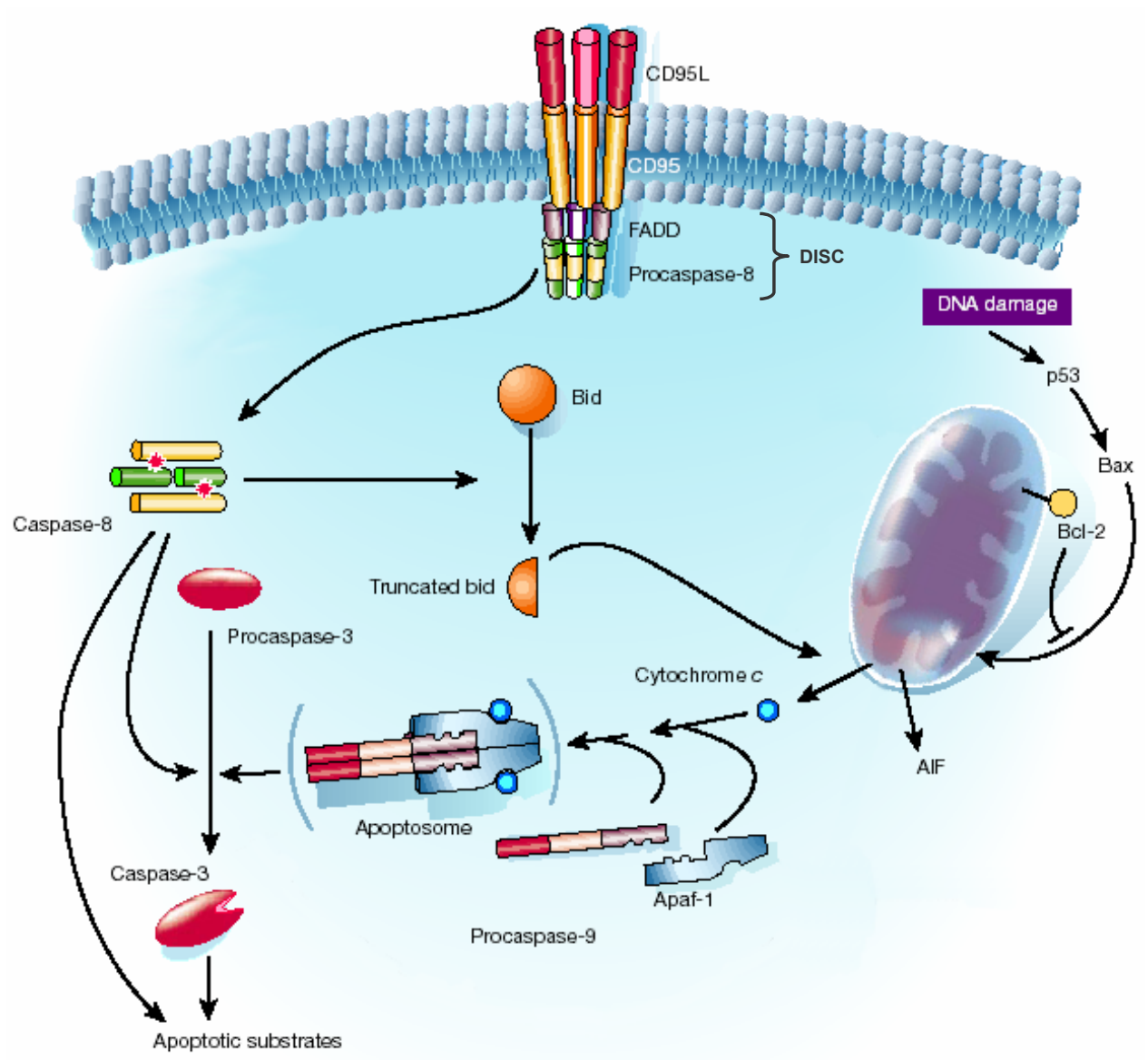

FIG. 1: Major apoptotic pathways. The extrinsic pathway of apoptosis induction is triggered by deathreceptors like CD95. Ligand (CD95L) binding induces receptor clustering and assembly of the DISC (deathinducing signalling complex). Upon interaction with the cytoplasmic receptor domain, the adapter protein FADD (Fas associated death domain protein) recruits multiple procaspase-8 molecules to the DISC. The high local concentration of procaspase- 8 leads to its dimerization-induced activation. Cellular stress like DNA-damage induces the intrinsic pathway and leads to $\mathrm{p} 53$-mediated transcriptional activation of pro-apoptotic Bcl-2 family members such as Bax. These proteins cause the release of other pro-apoptotic proteins like cytochrome $\mathrm{c}$ and AIF (apoptosisinducing factor) from the mitochondrial intermembrane space. Upon association with cytochrome c, Apaf-1 (apoptotic protease activation factor 1) oligomerizes and interacts with procaspase-9 to form the apoptosome representing the active form of caspase-9. Although the apoptosome is depicted here as a dimer it is a wheel-like complex with seven-fold symmetry (Acehan et al., 2002). Active caspase- 8 and -9 proteolytically activate the executioner caspases. Among these, caspase-3 cleaves various apoptotic substrates leading to regulated destruction of the cell. Extrinsic and intrinsic pathway are connected by the pro-apoptotic factor Bid. Caspase-8-mediated cleavage of Bid leads to translocation of truncated Bid to mitochondria where it induces cytochrome c release. (Please note that this figure represents a simplified overview of major apoptotic pathways and was modified according to Hengartner et al., 2000.)

Cellular stress like DNA damage intrinsically induces apoptosis by p53-mediated transcriptional activation of pro-apoptotic members of the Bcl-2 protein family such as Bax (reviewed in 
Hengartner, 2000; Rich et al., 2000). These proteins mediate the release of other pro-apoptotic molecules from the mitochondrial intermembrane compartment. Among them is cytochrome c which is responsible for the further transduction of the apoptotic signal.

Initiation of both pathways, extrinsic and intrinsic, leads to the activation of a proteolytic cascade which culminates in the disintegration of the whole cell. Members of the caspase protease family are the main executioners of these degradation processes.

\subsubsection{Caspases - central executioners of apoptosis}

Caspases constitute a family of cysteine proteases that are highly conserved through evolution. The family members share a conserved pentapeptide active-site-motif QACXG (where X can be R, Q or D) and specifically cleave their substrates C-terminal to an aspartate (Cohen, 1997). The substrate specificity of distinct caspases is conferred by the four amino acid residues N-terminal of the cleavage site (Thornberry et al., 1997). So far, 11 family members have been identified in humans. Among them, seven are involved in apoptosis, three are known for pro-inflammatory cytokine activation and one is involved in keratinocyte differentiation (Fuentes-Prior and Salvesen, 2004). Caspases are expressed as inactive zymogens (procaspases) which consist of an $\mathrm{N}$-terminal prodomain followed by a large (17-20 kDa) and a small domain (10-12 kDa) (Salvesen and Dixit, 1997). Active caspases are composed of two catalytic domains and each of them is derived from a single procaspase molecule and contains the large and the small subdomain (Fuentes-Prior and Salvesen, 2004).

The apoptotic caspases are grouped into initiator (upstream) and executioner (downstream) caspases depending on their position in signal transduction. These two groups differ in their prodomains and their activation mechanism. The initiator caspases 2, 8, 9 and 10 have a long prodomain containing either homotypic death effector domains (DED; caspase-8, -10) or caspase-recruitment domains (CARD; caspase-2, -9) (reviewed in Fuentes-Prior and Salvesen, 2004). These domains target procaspases to multiprotein complexes where their activation takes place. Procaspase- 8 is mainly activated by the extrinsic pathway (Fig. 1). Interaction of its DED with FADD leads to the recruitment of multiple procaspase- 8 molecules to the DISC where they are activated (Muzio et al., 1996; Vincenz and Dixit, 1997). Initially it was suggested that the high local concentration of procaspase- 8 in the DISC leads to proteolytic transactivation (Muzio et al., 1998; Salvesen and Dixit, 1999). However, more recent data point to an activation through procaspase- 8 dimerization induced at the DISC (Boatright et al., 2003; Donepudi et al., 2003). Autocatalytic cleavage then probably stabilizes the dimer and leads to the release of active caspase-8 into the cytoplasm (Chang et al., 2003). 
Intrinsic induction of apoptosis leads mainly to activation of procaspase-9 (Fig. 1). As mentioned above cellular stress causes the release of cytochrome $\mathrm{c}$ from mitochondria. The cytosolic apoptotic protease activation factor 1 (Apaf-1) interacts with cytochrome $\mathrm{c}$ and oligomerizes upon additional binding of ATP. Apaf-1 harbors a N-terminal CARD which interacts with the CARD in the prodomain of procaspase-9 (Acehan et al., 2002; Kothakota et al., 1997; Mashima et al., 1999). Thereby, a large protein complex named apoptosome is formed and represents the active caspase- 9 holoenzyme.

Once the initiator caspases are activated they proteolytically activate the executioner procaspase-3, -6 and -7 . Procaspase- 3 and -7 already form dimers before apoptosis induction and crystal structures of procaspase-7 and active caspase-7 suggest the following activation mechanism for executioner caspases (Chai et al., 2001; Riedl et al., 2001; Wei et al., 2000). Cleavage in the linker region between the small and large subunit leads to a rearrangement of catalytic residues and substrate binding residues creating the active catalytic site. Previously, Han et al. (1997) demonstrated that procaspase-3 is activated through two consecutive proteolytic steps in a cell free system. Procaspase-3 is cleaved in the linker region between the large p17 and smaller p12 subunit first and then the prodomain is removed. According to these studies, the second cleavage step is either driven autocatalytically or requires a caspase-3-like activity (Han et al., 1997).

Caspases are thought to be the executioners of apoptosis because they are responsible for most of the morphological changes observed in apoptotic cells (reviewed in Fischer et al., 2003; Hengartner, 2000). For example, cytoskeleton and structural proteins such as gelsolin (Geng et al., 1998; Kothakota et al., 1997), vimentin (Byun et al., 2001), fodrin (Cryns et al., 1996; Martin et al., 1995; Vanags et al., 1996) and paxillin (Shim et al., 2001) count to the caspase substrates and cleavage of these proteins is believed to play a role in cell shrinkage, cell detachment and membrane blebbing (Fischer et al., 2003). In addition, caspases cleave a number of nuclear proteins such as lamins (Rao et al., 1996), emerin (Columbaro et al., 2001) and nuclear scaffold attachment factors (Gohring and Fackelmayer, 1997). Degradation of these proteins is implicated in the disassembly of the nuclear lamina and nuclear envelope breakdown. Another nuclear event is the apoptotic DNA fragmentation, resembling one of the biochemical hallmarks of apoptosis.

\subsubsection{DNA fragmentation during apoptosis}

Two different stages of DNA fragmentation are observed in most apoptotic cells (Cohen and Duke, 1984; Wyllie, 1980). First the chromosomal DNA is cleaved into high molecular weight (HMW) fragments with a size of $50-300 \mathrm{kbp}$. In the second step they are further cut into 
fragments of mononucleosomal and oligonucleosomal size. Oligonucleosomal DNA fragmentation was initially reported in 1980 as the consequence of a glucocorticoid treatment of mouse thymocytes (Wyllie, 1980). During this treatment DNA complexes containing multimers of about $180 \mathrm{bp}$ of double-stranded DNA were released from the nucleus. These results suggested that upon induction of apoptosis a double-strand specific endonuclease is activated that mediates internucleosomal DNA cleavage. Several nucleases such as DNase I, DNase II, DNase $\gamma$ and cyclophilins were proposed to be involved in the internucleosomal DNA fragmentation. However, today it is generally accepted that this cleavage is mainly caused by the DNA fragmentation factor (DFF) 40 (reviewed in Nagata et al., 2003; Zhivotovsky et al., 1994).

\subsubsection{The DNA fragmentation factor (DFF)}

The $40 \mathrm{kDa}$ endonuclease DFF40 was initially isolated from HeLa cells, mouse lymphoma cells and human Jurkat cells by different groups, and it was also designated as caspase-activated DNase (CAD) and caspase-activated nuclease (CPAN) (Enari et al., 1998; Halenbeck et al., 1998; Liu et al., 1997). Additionally, DFF40 was identified in rat, chicken, zebrafish and Drosophila (reviewed in Nagata et al., 2003). In proliferating cells DFF40 is expressed in the presence of the $45 \mathrm{kDa}$ protein DFF45 (also named inhibitor of CAD, ICAD) which has a dual role as chaperone and inhibitor of DFF40 (Enari et al., 1998; Halenbeck et al., 1998; Sakahira et al., 2000). Besides DFF45 the general chaperones Hsp70 and Hsp40 are additionally involved in the correct folding of DFF40 (Sakahira and Nagata, 2002).

A structural feature of both DFF40 and DFF45 is the N-terminal CIDE (cell death-inducing DFF45-like effector) domain which is homologous to the N-terminus of the members of the proapoptotic CIDE protein family (Inohara et al., 1999). The CIDE domains of DFF40 and DFF45 are involved in their protein-protein interaction and directly bind to each other (Inohara et al., 1999; Otomo et al., 2000; Zhou et al., 2001). However, other domains of DFF45 are required for its effective chaperone function ( $\mathrm{Gu}$ et al., 1999; Sakahira et al., 1999). Although DFF was initially thought to be a heterodimer (Liu et al., 1997; Widlak et al., 2003), recently it was shown that the complex probably contains two heterodimers each composed of a DFF40 and a DFF45 subunit (Lechardeur et al., 2005). In addition, this study demonstrated that the CIDE domain of DFF40 is not necessary for the DFF40/DFF45 dimerization but that it is required for the formation of the tetramer. This suggests that the interaction of two DFF40/DFF45 dimers is mediated, at least in part, by the CIDE domains of DFF40 (Lechardeur et al., 2005).

In human, mouse, and rat the DFF45 mRNA is alternatively spliced leading to an additional shorter (35 kDa) isoform which is called DFF35 or ICAD-S (Enari et al., 1998; Gu et al., 1999). 
Both DFF45 and DFF35 inhibit the nuclease activity of DFF40, however, only DFF45 fulfills the function as chaperone of DFF40. Therefore, DFF35 is not included in DFF complexes in proliferating cells (Gu et al., 1999; Sakahira et al., 1999).

During apoptosis caspase-3 and caspase-7 cleave DFF45 at two sites, aspartate 117 and 224, thereby releasing DFF40 (Liu et al., 1999; Sakahira et al., 1998). In addition, the protease granzyme B which plays an important role in cytotoxic $\mathrm{T}$ lymphocyte mediated apoptosis was shown to cleave DFF45 directly leading to caspase-independent DNA fragmentation (SharifAskari et al., 2001; Thomas et al., 2000). Structural and biochemical data suggest that activated DFF40 molecules dimerize (Korn et al., 2005; Lechardeur et al., 2005). This dimerization additionally requires the presence of $\mathrm{Zn}^{2+}$, however, a non-physiological excess of $\mathrm{Zn}^{2+}$ inhibits the nuclease (Woo et al., 2004). Dimerization presumably creates a pair of molecular scissors with a deep active site crevice (Woo et al., 2004). A model for the DNA binding to the DFF40 dimer shows that the DNA has to approach the bottom of the crevice to reach the active site. This picture easily explains why DFF40 cleaves DNA in the internucleosomal linker regions; simply because histone associated DNA packed in a nucleosome is not flexible enough to reach the catalytic residues (Woo et al., 2004). These include several essential histidine, lysine and tyrosine residues in the C-terminal domain of DFF40 that are conserved among different species (Korn et al., 2002; Meiss et al., 2001; Sakahira et al., 2001). The enzymatic properties of DFF40 were mainly characterized for human and mouse nucleases. These cleave double-stranded DNA but not single-stranded DNA or RNA in the presence of $\mathrm{Mg}^{2+}$ at neutral $\mathrm{pH}$ (Widlak et al., 2000). A/T-rich DNA regions are preferred and mainly DNA fragments with blunt ends and a 5'-phosphate and 3'-hydroxyl group are generated (Widlak et al., 2000). In vitro studies demonstrated that direct binding of certain proteins like histone $\mathrm{H} 1$, high mobility group protein (HMG)-1, HMG-2 and topoisomerase II $\alpha$ to active DFF40 further enhance its activity (Durrieu et al., 2000; Liu et al., 1998; Liu et al., 1999; Widlak et al., 2005; Widlak et al., 2000). However, the relevance of these interactions has still to be elucidated in vivo.

Although the intracellular function of DFF40 is clearly nuclear the DFF complex was initially identified in the cytoplasm of mammalian cells (Enari et al., 1998; Liu et al., 1997). These findings together with a sequence analysis based prediction of a nuclear localization signal (NLS) at the very C-terminus of mouse DFF40 led to the following activation model (Enari et al., 1998): The DFF complex is retained in the cytoplasm of healthy cells because DFF45 masks the putative DFF40 NLS. During apoptosis DFF40 is released from cleaved DFF45 leading to exposition of the NLS and to nuclear transport of activated DFF40. In contrast to this model, other groups observed a dominant nuclear localization of endogenous mouse DFF45 as well as 
overexpressed DFF subunits in healthy cells (Lechardeur et al., 2000; Liu et al., 1998; Samejima and Earnshaw, 1998). Therefore, it is meanwhile accepted that the DFF complex is translocated into the nucleus in healthy cells where DFF40 has to be activated once the cell undergoes apoptosis. Besides the putative NLS in the C-terminus of DFF40 an additional NLS was predicted in the C-terminal sequences of human and mouse DFF45 (Lechardeur et al., 2000). Nuclear localization studies showed that the C-terminal regions of both DFF40 and DFF45 are involved in the nuclear accumulation of the DFF complex (Lechardeur et al., 2000; Samejima and Earnshaw, 2000). However, the nuclear transport mechanism has not been characterized so far.

\subsubsection{Additional apoptotic nucleases}

Apoptotic DNA fragmentation was not only observed in cells undergoing apoptosis but DNA is additionally degraded in lysosomes of macrophages and surrounding cells that engulf apoptotic bodies (McIlroy et al., 2000; Odaka and Mizuochi, 2002). This lysosomal DNA degradation was observed in transgenic mice defective in DFF40-mediated DNA fragmentation, and the responsible nuclease is probably DNase II (McIlroy et al., 2000).

Knock-out mice deficient in DFF40 or DFF45 (which as a chaperone is indispensable for the expression of functional DFF40) are viable and do not exhibit adverse phenotypes (Nagata et al., 2003). Most of the cells derived from these knock-out mice do not show apoptotic DNA fragmentation (Zhang et al., 1998; Zhang et al., 1999). However, residual and delayed DNA fragmentation could be observed in these animals suggesting that additional apoptotic endonucleases exist ( $\mathrm{Li}$ et al., 2001; Yakovlev et al., 2001). As a result endonuclease G was identified which is located in the mitochondrial intermembrane space and is released upon induction of apoptosis ( $\mathrm{Li}$ et al., 2001; van Loo et al., 2001). Endonuclease G is G/C-specific (Ruiz-Carrillo and Renaud, 1987) and induces preferentially single-stranded nicks not only in internucleosomal regions but also within nucleosomes (Widlak et al., 2001). Since its enzymatic properties differ from DFF40, endonuclease G alone can not be responsible for the observed apoptotic DNA fragmentation (Widlak et al., 2001).

As mentioned earlier, internucleosomal DNA cleavage is preceded by the generation of HMW DNA fragments (Lagarkova et al., 1995; Oberhammer et al., 1993). Because DFF45-deficient cells lack both fragmentation stages it was proposed that DFF40 is also responsible for the HMW degradation (Boulares et al., 2001; Zhang et al., 2000). However, in some cell lines only HMW DNA fragmentation was observed rather indicating that the two fragmentation steps are carried out by different nucleases (Oberhammer et al., 1993; Samejima et al., 2001). Additionally, the 
apoptosis-inducing factor (AIF) was considered to be involved in the first stage of DNA fragmentation (Susin et al., 1999) but a nuclease activity could not be demonstrated so far. Therefore, the role of AIF with regard to DNA fragmentation remains unclear.

\subsubsection{Functional relevance of apoptotic DNA fragmentation}

DFF40-mediated DNA fragmentation may be considered as a means to prevent gene transfer of potentially harmful DNA derived from tumor or virally-infected cells (reviewed in Widlak and Garrard, 2005; Zhang and Xu, 2002). Surprisingly, DFF40 and DFF45 knock-out mice are viable and the lack of DNA fragmentation does not affect the overall progression of apoptosis (Nagata et al., 2003). Therefore, apoptotic DNA fragmentation seems not to be essential for apoptosis and animal development. Contrary to DFF40-deficient mice, DNase II knock-out mice die at a fetal stage because of the failure of erythropoiesis and defects in thymus development. Furthermore, upon apoptosis in certain tissues undigested DNA accumulates in the macrophages of these embryos (Kawane et al., 2001; Krieser et al., 2002). In comparison, DNase II-mediated DNA degradation might be more important than internucleosomal DNA fragmentation (Nagata et al., 2003).

However, recently a connection between DNA fragmentation and tumor development was suggested because tumor-specific DFF45 gene mutations were described in human germ cell tumors and neuroblastoma tumors (Abel et al., 2002; Abel et al., 2004). This assumption was further supported by a report from Yan and colleagues demonstrating that DFF40-mediated DNA fragmentation is required for the maintenance of genomic stability and prevention of tumorigenesis (Yan et al., 2006a; Yan et al., 2006b).

\subsection{Nucleocytoplasmic transport}

The organization of eukaryotic cells includes different compartments. This compartmentalization spatially restricts cellular processes, but provides additional facilities for regulation. However, the fundamental process of gene expression (transcription in the nucleus and translation in the cytoplasm) for instance requires the exchange of macromolecules between compartments. Nucleus and cytoplasm are connected through the nuclear pore complexes (NPCs) that are embedded in the nuclear membrane. The NPC allows for passive diffusion of small molecules like ions but restricts the passage of macromolecules larger than 40-60 $\mathrm{kDa}$ which have to be actively transported. However, also molecules with a size below the diffusion limit often traverse the NPC in the active mode because this allows for highly efficient and regulated transport (reviewed in Conti and Izaurralde, 2001; Fried and Kutay, 2003; Görlich and Kutay, 1999). 
Soluble transport receptors often mediate the translocation processes. They specifically recognize transport signals within their cargo proteins and facilitate the passage through the NPC. Active transport allows for accumulation of molecules against their concentration gradient and therefore requires the input of energy.

\subsubsection{The nuclear pore complex (NPC)}

The NPC represents one of the largest macromolecular structures in eukaryotic cells with a mass of about $50 \mathrm{MDa}$ in yeast and $125 \mathrm{MDa}$ in vertebrates (Fahrenkrog et al., 2001; Rout and Aitchison, 2001; Weis, 2003). Although NPCs largely differ in mass the overall structure seems to be well conserved between species (Fahrenkrog et al., 1998). Electron microscopy of NPCs derived from yeast (Yang et al., 1998) and Xenopus (Akey and Radermacher, 1993; Hinshaw et al., 1992; Stoffler et al., 2003) revealed their three-dimensional structure (Fig. 2).

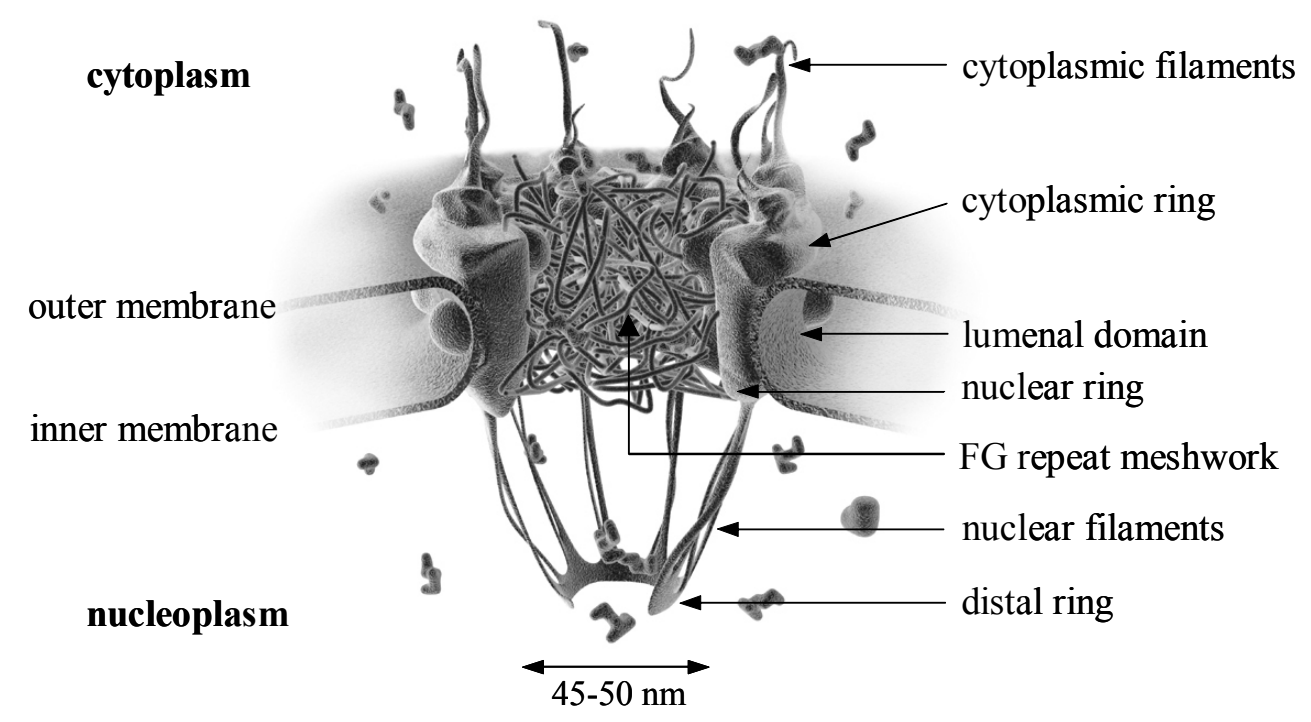

FIG. 2: The nuclear pore complex. Electron microscopy studies gave rise to a three-dimensional model of the NPC exhibiting octagonal symmetry. The main part of the pore forms a cylindrical structure embedded in the nuclear envelope which is sandwiched between a cytoplasmic and a nuclear ring. These ring structures are anchoring sites for cytoplasmic and nuclear filaments. The latter are not flexible but connected by a distal ring, therby forming the nuclear basket. The central channel of the pore is filled with FG repeats which form a meshwork through hydrophobic interactions (Frey et al., 2006) thereby creating the permeability barrier of NPCs. According to the selective-phase model (Ribbeck and Görlich, 2001), transport receptors transiently dissolve the meshwork through interactions with the FG repeats. (The NPC picture used in this figure was created by Samir S. Patel, http://sspatel.googlepages.com/nuclearporecomplex2). 
According to these studies the NPC exhibits an eight-fold symmetry and consists of a cylindrical structure composed of spoke-ring complexes. This central framework is sandwiched between cytoplasmic and nuclear ring structures which are the anchoring sites for eight cytoplasmic and eight nuclear filaments, respectively. While the cytoplasmic fibrils are flexible the nuclear filaments are connected by a distal ring, thereby the nuclear basket is formed. The central pore encircled by the spoke complexes comprises $45-50 \mathrm{~nm}$ at its narrowest position (Fahrenkrog and Aebi, 2003; Stoffler et al., 2003) and allows the passage of macromolecules with a diameter of up to $35-40 \mathrm{~nm}$ (or about $50 \mathrm{MDa}$ ) (Pante and Kann, 2002). Despite its large size the NPC consists of only about 30 different proteins in yeast and mammals, named nucleoporins (Cronshaw et al., 2002; Rout et al., 2000). Reflecting the eight-fold symmetry of the NPC each nucleoporin is present in a copy number of eight or multiples of eight. Approximately one third of the core nucleoporins are enriched in phenylalanine-glycine (FG) repeats (Cronshaw et al., 2002; Rout et al., 2000). These distinct FG domains have up to 50 repeat units (Rout and Wente, 1994) and provide important binding sites for transport receptors (Bayliss et al., 2000b; Fribourg et al., 2001).

Based on the interaction between transport receptor and nucleoporin different models for facilitated NPC passage were proposed. According to the "virtual gating" model (Rout et al., 2000) the NPC channel consists of a narrow central tube and the interaction with peripheral FGrich nucleoporins facilitates the entry of cargo-transport receptor complexes. The translocation through the NPC occurs by Brownian motion. The "selective phase" model (Ribbeck and Görlich, 2001) was initially based on kinetic data and proposes the existence of a hydrophobic meshwork in the central channel of the NPC formed by weakly interacting FG repeats. Upon interactions between transport receptors and FG repeats the sieve-like structure of the permeability barrier is transiently dissolved thereby allowing translocation of the transport receptors and their cargoes. The "oily spaghetti" model (Macara, 2001) also suggests that the central channel of the NPC is filled with FG repeats which repel molecules that should be excluded from passage. But in contrast to the "selective phase" model the clusters of hydrophobic amino acids within the repeats do not interact with each other. Recent data clearly showed that FG-rich repeats indeed form a three-dimensional meshwork with hydrogel-like properties strongly supporting the "selective phase" model (Frey et al., 2006). Accordingly, the size limit of the molecular sieve is overcome by disruption of adjacent inter-repeat cross-links (Frey et al., 2006). 


\subsubsection{Nuclear transport receptors}

Soluble transport receptors are among the key components of the process of nucleocytoplasmic transport because they mediate most nuclear transport events. The largest class of nuclear transport receptors is the superfamily of importin $\beta$-like proteins also named karyopherins. Depending on their function in nuclear import or export they are referred to as importins or exportins (reviewed in Weis, 2003, and see references therein). In higher eukaryotes the importin $\beta$ family comprises more than 20 members that are characterized by an $\mathrm{N}$-terminal RanGTP binding domain, a high proportin of hydrophobic residues, an acidic isoelectric point from 4.6 to 5.9, and a large molecular weight of 90 to $145 \mathrm{kDa}$ (Fried and Kutay, 2003; Görlich et al., 1997). So far, ten of these transport receptors were identified as importins (importin $\beta$, transportin, transportin 2, transportin SR, importin 4, importin 5, importin 7, importin, 8, importin 9, importin 11), seven facilitate nuclear export (Crm1/exportin1, CAS, exportin-t, exportin 4, exportin 5, exportin 6, exportin 7) and exportin 13 is able to mediate bi-directional transport (reviewed in Fried and Kutay, 2003; Pemberton and Paschal, 2005). Regarding the large number of proteins that have to be transported into and out of the nucleus the number of transport factors is very low; therefore, most of them probably recognize many different cargoes. The substrate spectrum is additionally enlarged since some importins and exportins not only bind their cargoes directly but also use adapter proteins for cargo recognition (Fried and Kutay, 2003; Weis, 2003). Among them, the importin $\beta$ adapter molecule importin $\alpha$ is best characterized. In humans there are six importin $\alpha$ variants which show over $60 \%$ sequence similarity and can be assigned to three subclasses. Importin $\alpha 1$ (NPI-1), importin $\alpha 6$ and importin $\alpha 7$ are members of the NPI-1 class; importin $\alpha 2$ (Rch-1) represents the class of Rch-1 receptors; and importin $\alpha 3$ and importin $\alpha 4$ (Qip1) belong to the Qip1 class (Kohler et al., 1997; Miyamoto et al., 1997). The importin $\alpha$ isoforms have distinct binding properties (Jans et al., 2000). For instance the transcription factors STAT (signal transducers and activators of transcription) 1 and 2 interact with importin $\alpha 1$ but not with importin $\alpha 2$. Cargo binding to importin $\alpha 3$ requires additional amino acid residues flanking the basic NLS (Jans et al., 2000; Miyamoto et al., 1997). All importin $\alpha$ subtypes share a large nuclear localization signal (NLS) binding domain and a flexible N-terminal importin $\beta$-binding (IBB) domain (Görlich et al., 1996a; Weis et al., 1996b). The NLS binding domain comprises ten tandem armadillo (ARM) repeats which consist of about 40 amino acid residues folded into three $\alpha$ helices (Peifer et al., 1994) (Fig.3). The ARM repeats are arranged in an almost parallel fashion forming an elongated helical surface groove which contains an array of binding pockets (Conti et al., 1998; Fontes et al., 2000; Kobe, 1999). Each of these binding pockets is created by a pair of conserved tryptophan (Trp) and asparagine (Asn) 


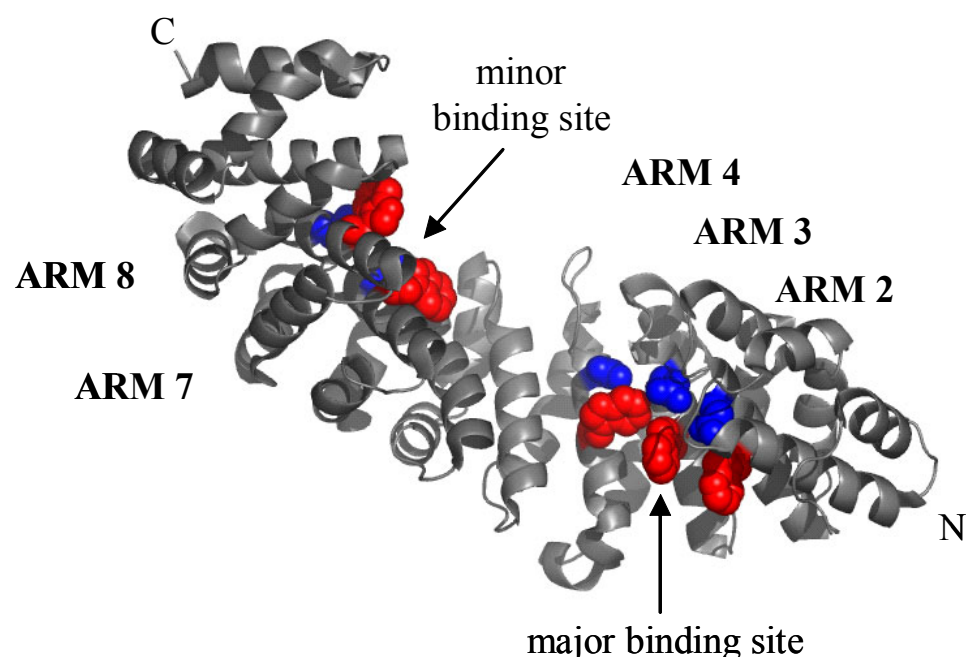

FIG. 3: Structure of the nuclear transport adapter importin $\alpha$. Ribbon representation of the ARM domain of mouse importin $\alpha$ (accession number 1EJL, RCSB protein data bank) generated using the program PYMOL. The ARM domain comprises ten armadillo repeats each of them consisting of three $\alpha$-helices. Conserved tryptophan (red) and asparagine (blue) pairs in ARM repeats 2-4, 7 and 8 are indicated. Each tryptophan-asparagine pair creates a binding pocket. The ladder of binding pockets is interrupted in ARM repeats 5 and 6 , thereby forming minor binding site (ARM 7-8) and major binding site (ARM 2-4) for classical NLSs.

residues located in helix 3 of ARM repeats 2-4, 7 and 8. The ladder of Trp-Asn pairs is interrupted in ARM repeats 5 and 6, thereby ARM repeats 2-4 create a larger N-terminal major binding site while ARM repeats 7 and 8 form a smaller C-terminal minor binding site (reviewed in Conti, 2002). The very N-terminal IBB domain comprises the first 44 amino acid residues and is enriched in arginines and lysines (Görlich et al., 1996a; Weis et al., 1996b). In the absence of importin $\beta$ a small part of this domain functions as autoinhibitory segment through interaction with the larger NLS binding site of importin $\alpha$ (Kobe, 1999). Binding of importin $\beta$ to the IBB domain abolishes the autoinhibition and promotes the interaction between importin $\alpha$ and cargo proteins. The binding of the IBB domain to importin $\beta$ resembles the direct importin $\beta$-cargo interaction in the absence of an adapter molecule (Henderson and Percipalle, 1997; Truant and Cullen, 1999).

Studies regarding the structures of $\beta$-family members mainly addressed importin $\beta$ (reviewed in Madrid and Weis, 2006) and revealed a superhelical conformation with topological similarity to importin $\alpha$ (Conti, 2002). Importin $\beta$ consists of 19 HEAT repeats (Fig. 4A) that are named after the proteins in which the motif was first discovered, namely Huntingtin, Elongation factor 3, 'A' subunit of protein phosphatase A, and TOR1 lipid kinase (Andrade and Bork, 1995). Each HEAT repeat is composed of two $\alpha$-helices (A and B) connected by a short loop. ARM and 
HEAT repeats are very similar as indicated by superposition of the individual structures suggesting that proteins containing these motifs belong to the same protein superfamily (Cingolani et al., 1999; Malik et al., 1997). The convex outer surface of importin $\beta$ is formed by the A helices while the B helices form the concave inner face (Cingolani et al., 1999) (Fig. 4B).
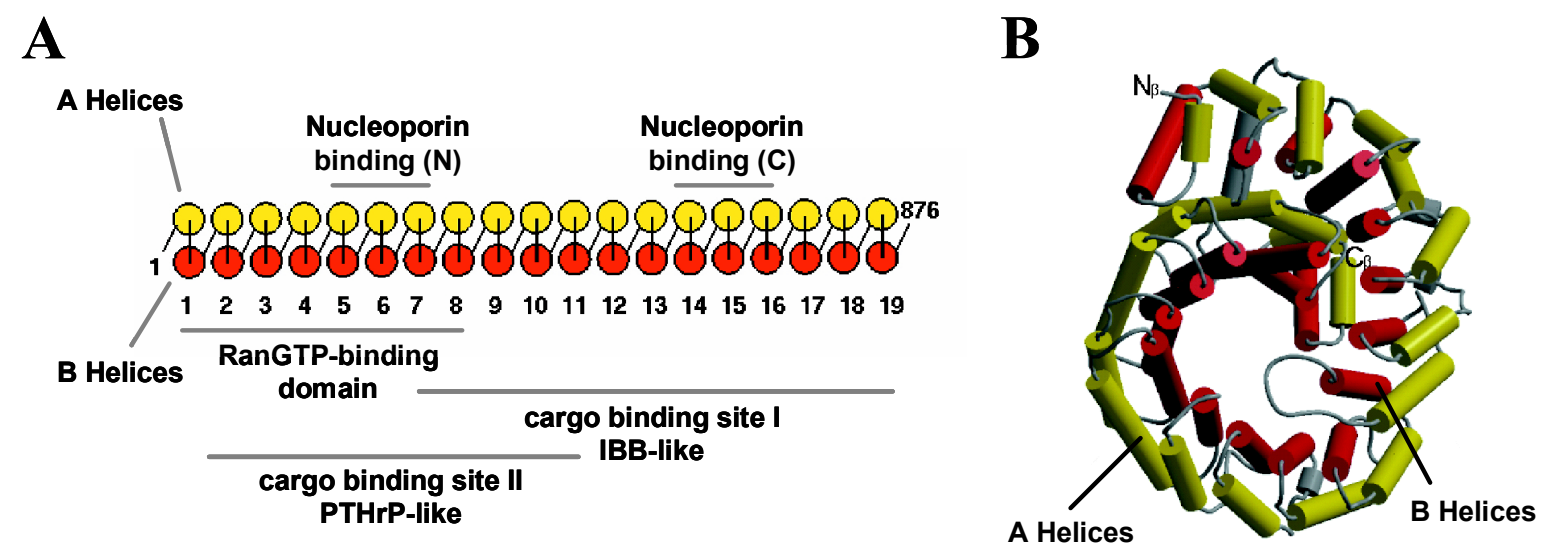

FIG. 4: The nuclear transport receptor importin $\boldsymbol{\beta}$. $A$, domain organization. Importin $\beta$ contains 19 HEAT repeats each consisting of two $\alpha$-helices, A (yellow) and B (red), connected by a short loop. Binding sites for nucleoporins, RanGTP and cargoes are indicated (for more details, see text). $B$, three-dimensional structure of importin $\beta$. The composition of HEAT repeats results in a superhelical spring-like structure which confers a high flexibility and enables importin $\beta$ to recognize various cargo proteins. A helices (yellow rods) form the convex outer surface of importin $\beta$ while B helices generate the concave inner face. [This figure was partially adopted from a figure generated by Bednenko et al. (2003).]

FG repeats of nucleoporins interact with the outer surface (Bayliss et al., 2000b) whereas RanGTP (HEAT 1-10) (Vetter et al., 1999), the importin $\alpha$ adapter, and cargo bind to the inner site. The IBB domain of importin $\alpha$ associates with HEAT repeats 7-19 (cargo binding site I), a region that is also directly recognized by cargo proteins (Cingolani et al., 1999). In addition, Cingolani et al. (2002) identified a second binding site (cargo binding site II) that is located to HEAT repeats 2-11.

Additional structural studies suggest that all members of the importin $\beta$ family are composed of 18-20 HEAT repeats forming superhelical molecules (Chook and Blobel, 1999; Matsuura and Stewart, 2004; Petosa et al., 2004). These spring-like structures confer a high flexibility that enables the nuclear transport receptors to recognize a large variety of cargo proteins (Conti et al., 2006; Stewart, 2003). 


\subsubsection{Nuclear transport signals}

There are two types of transport signals recognized by nuclear transport receptors. Proteins carrying a nuclear localization signal (NLS) are recognized by importins while proteins containing a nuclear export signal (NES) are labeled for nuclear export (Fried and Kutay, 2003). NLSs can be generally categorized in classical (cNLS) and non-classical (ncNLS) signals depending on the cargo-receptor interaction mode. The nuclear transport of proteins containing a cNLS is exclusively mediated by importin $\beta$; however, its binding requires the importin $\alpha$ adapter protein (Görlich et al., 1995a; Mattaj and Englmeier, 1998). In contrast, proteins harboring ncNLSs are directly recognized by importin $\beta$-family receptors (Strom and Weis, 2001).

Classical NLS are generally characterized as short stretches enriched in positively charged amino acids (Dingwall and Laskey, 1991; Robbins et al., 1991) and can be classified as monopartite and bipartite cNLSs. Monopartite cNLS contain one cluster of positively charged amino acids as in the case of the SV40 large T antigen (Kalderon et al., 1984a). Bipartite cNLS like that of nucleoplasmin are composed of two basic regions usually connected by a 10-12 amino acid spacer (Dingwall et al., 1988; Robbins et al., 1991). Several crystal structures of importin $\alpha$ bound to cNLS confirmed that monopartite cNLSs and the larger downstream basic cluster of bipartite cNLSs bind to the major binding site of importin $\alpha$ (reviewed in Conti, 2002; Madrid and Weis, 2006). The smaller upstream basic cluster of bipartite cNLSs binds to the minor binding site of importin $\alpha$ (Conti, 2002; Madrid and Weis, 2006). The conserved Trp-Asn pairs represent a recognition motif for the lysine $(\mathrm{K})$ and arginine $(\mathrm{R})$ residues of cNLSs and the binding is mediated by polar, hydrophobic and electrostatic interactions (Conti, 2002). Additionally, the structural data lead to a modification of the originally defined consensus sequences for cNLS. Accordingly, the current view of a monopartite cNLS follows the consensus K-K/R-X-K/R (Chelsky et al., 1989). Amino acid residues that are compatible with sharp turns like proline or glycine upstream of the consensus motif and a small hydrophobic amino acid followed by an acidic residue downstream of the consensus sequence are preferred (Conti, 2002). In contrast to the monopartite cNLS, the sequence requirements for bipartite cNLS are less stringent because of multiple interactions between NLS and importin $\alpha$ surface groove (Conti and Kuriyan, 2000; Dingwall and Laskey, 1991). Bipartite cNLSs follow the loose

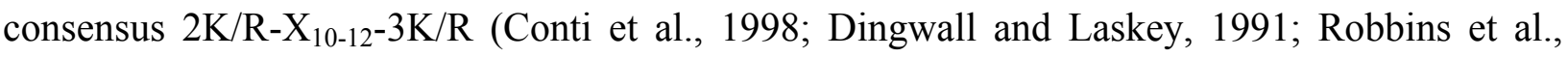
1991). However, exceptions of this consensus motif regarding the spacer length were reported. Makkerh et al. (1996) demonstrated already that the nucleoplasmin NLS is still able to mediate nuclear import when the spacer is mutated and its length is increased to 20 alanine residues. 
Additionally, in certain cases such as for the heterogenous nuclear ribonucleoprotein (hnRNP) type 1 the spacer might reach a length of up to 32 amino acids (Romanelli and Morandi, 2002). In contrast, shortening of the spacer will prevent the interaction with importin $\alpha$ because the NLS is not longer able to span the physical separation between major and minor binding site of importin $\alpha$ (Conti, 2002). Moreover, recently a bipartite cNLS was described that is not composed of two basic stretches but is scattered over a region of 91 amino acid residues (Rodriguez et al., 2006). In the three-dimensional structure the basic amino acids are clustered and resemble the topological disposition of a bipartite cNLS.

Non-classical NLS are less well defined than cNLS but were mapped for some proteins (reviewed in Pemberton and Paschal, 2005). In the case of core histones and ribosomal proteins ncNLSs are highly enriched in basic amino acids (Baake et al., 2001b; Jakel and Görlich, 1998; Mosammaparast et al., 2002; Mosammaparast et al., 2001; Muhlhausser et al., 2001). In contrast, the M9 domain of hnRNP A1 for instance is relatively large and contains glycine rather than positively charged amino acids (Pollard et al., 1996). The analysis of non-classical nuclear transport pathways often revealed a significant redundancy. In other words, the nuclear transport of certain cargoes can be mediated by different import receptors (Baake et al., 2001a; Jakel and Görlich, 1998; Mosammaparast et al., 2002; Mosammaparast et al., 2001; Muhlhausser et al., 2001).

The interaction between export receptors and their cargo proteins is mediated by NESs. Among these export signals the hydrophobic NES is best characterized. This signal consists of a short leucine-rich sequence following a loose consensus (Bogerd et al., 1996; Kutay and Guttinger, 2005). This kind of signal was first identified in the protein kinase A inhibitor (Wen et al., 1995) and HIV Rev (Fischer et al., 1995). Leucine-rich NESs are recognized by the most versatile export receptor Crm1/exportin 1 (Fornerod et al., 1997; Stade et al., 1997) that mediates nuclear export of RNA binding proteins, translation factors, and various shuttling proteins such as cell cycle regulators and transcription factors (reviewed in Fornerod and Ohno, 2002; Fried and Kutay, 2003). However, Crm1 can also recognize proteins that do not exhibit the short hydrophobic NES. An example is the importin $\beta$ adapter snurportin that interacts with Crm1 via a large domain that contains only a few dispersed leucines (Paraskeva et al., 1999). Besides Crm1 other nuclear export receptors exist (compare chapter 1.2.2) that do not recognize leucinerich NESs. For the majority of these exportins only a small number of substrates have been identified. Nevertheless, exportin 7 also appears to be a nuclear export factor with a broad substrate specificity (Mingot et al., 2004). NESs recognized by exportin 7 largely differ from Crm1 specific NESs since they include folded motifs and positively charged amino acid residues 
(Mingot et al., 2004). Additionally, exportin CAS is very important because it facilitates the export of importin $\alpha$ and therefore ensures the classical protein import cycle (Kutay et al., 1997a).

\subsubsection{The small GTPase Ran and its function in nuclear transport}

Since receptor mediated nucleocytoplasmic transport occurs against concentration gradients it is an energy-dependent process (reviewed in Fried and Kutay, 2003; Weis, 2003). However, the energy which derives from GTP hydrolysis is not necessary for the translocation of cargoreceptor complexes through the nuclear pore, but determines its direction. GTP hydrolysis is mediated by Ran, a member of the Ras-related GTPase superfamily that cycles between a GTPand a GDP-bound state. RanGTP is highly concentrated in the nuclear compartment and very low concentrated in the cytoplasm. This asymmetric distribution is responsible for the association and dissociation of cargo-receptor complexes and therefore confers the directionality of nucleocytoplasmic transport (Görlich et al., 1996b; Izaurralde et al., 1997). The RanGTP gradient across the nuclear membrane is maintained by additional factors. The exclusively cytoplasmic GTPase-activating protein (RanGAP) stimulates the GTPase activity of Ran together with Ran binding protein (RanBP) 1 and 2 (Bischoff et al., 1994; Bischoff et al., 2002). RanGAP is restricted to the cytoplasm through (i) interaction with the cytoplasmic nucleoporin RanBP2 (Mahajan et al., 1997; Matunis et al., 1996) and (ii) nuclear export by Crm1 (Mingot et al., 2004, and references therein). In contrast, Ran loading with GTP solely occurs in the nucleus and is mediated by the chromatin-bound guanine nucleotide exchange factor RanGEF (RCC1) (Klebe et al., 1995). The role of RanGTP in terms of regulating nuclear transport processes is exemplified by the classical nuclear import pathway (Fig. 5) (reviewed in Fried and Kutay, 2003): In the absence of RanGTP the trimeric complex consisting of cargo, importin $\alpha$ and importin $\beta$ assembles in the cytoplasm (Weis et al., 1996a) and traverses the nuclear membrane through interactions between importin $\beta$ and nucleoporins (Bayliss et al., 2000a; Görlich et al., 1996b; Rexach and Blobel, 1995). In the nucleus, RanGTP binds to the N-terminal domain of importin $\beta$ leading to conformational changes of importin $\beta$ and therefore to the disassembly of the trimeric import complex (Lee et al., 2000). Importin $\beta$ bound to RanGTP reenters the cytoplasm through NPCs while importin $\alpha$ has to be actively exported from the nucleus. In contrast to import receptors, exportins bind their cargoes only in the presence of RanGTP (Görlich et al., 1996b; Izaurralde et al., 1997). Therefore, the importin $\alpha$ specific exportin CAS recognizes its target only in the RanGTP bound state (Kutay et al., 1997a). Once the trimeric export complex composed of CAS, RanGTP, and importin $\alpha$ is formed, CAS facilitates exit from 


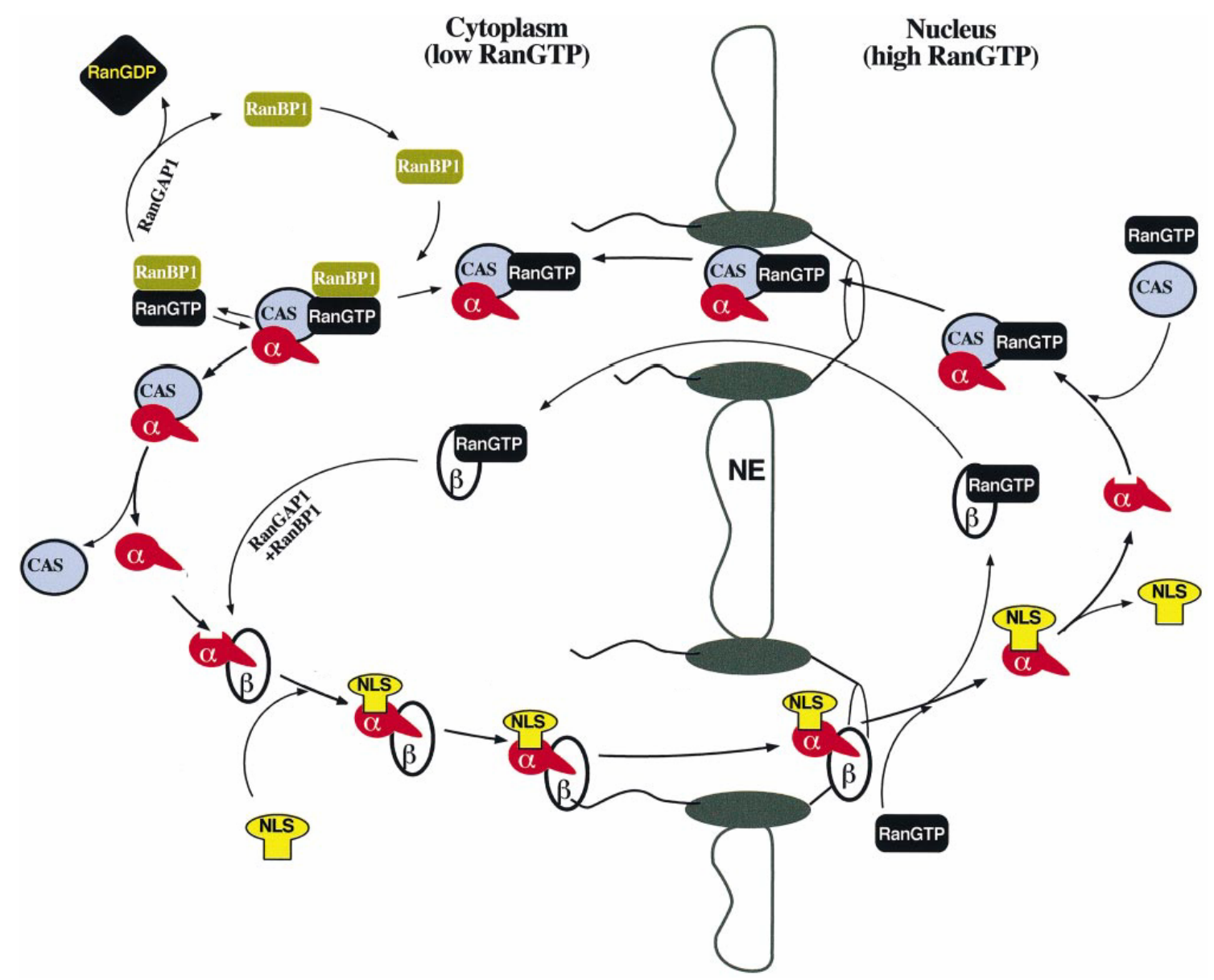

FIG. 5: The classical nuclear import cycle. Model for importin $\alpha / \beta$-mediated nuclear transport. The directionality of nucleocytoplasmic transport is determined by assymetric distribution of RanGTP with a high concentration in the nucleus and a low concentration in the cytoplasm. In the absence of RanGTP importin $\alpha$ associates with importin $\beta$ and binds to its cargo protein (depicted as NLS). Translocation of this trimeric complex through the NPC is mediated by interactions between importin $\beta$ and FG repeats of nucleoporins. In the nucleus RanGTP binds to importin $\beta$ leading to disassembly of the import complex. CAS binds to released importin $\alpha$ in the presence of RanGTP and promotes its export from the nucleus. In contrast, importin $\beta$ bound to RanGTP traverses the NPC on its own. In the cytoplasm RanGAP together with RanBP1/2 stimulates the GTPase activity of Ran. Upon GTP hydrolysis importin $\beta$ dissociates from Ran and the importin $\alpha$-CAS-Ran complex disassembles. Released importin $\alpha$ and importin $\beta$ are available for another transport cycle. [This figure was taken from Görlich (1998)]

the nucleus. Upon GTP hydrolysis in the cytoplasm importin $\beta$ dissociates from RanGDP while importin $\alpha$ is released from CAS in the presence of RanGDP. Both molecules, importin $\alpha$ and importin $\beta$, can now enter another import cycle. RanGDP is imported into the nucleus by its specific import factor NTF2 that is not related to the $\beta$-family receptors (Ribbeck et al., 1998; Smith et al., 1998). In the nucleus RanGEF induces the dissociation of this cargo complex and reloads Ran with GTP thereby maintaining the RanGTP gradient across the nuclear membrane. 


\subsection{Nucleocytoplasmic transport in apoptosis}

During apoptosis various nuclear components are cleaved which ultimately lead to the nuclear breakdown. Hence, apoptotic factors such as executioner caspases have to enter the nucleus. On the other hand, DNA damage triggers apoptosis in the nucleus and this apoptotic signal has to be transduced into other cellular compartments to ensure the coordinated execution of cell death. In conclusion, nucleocytoplasmic transport of signal transducers and execution factors is probably crucial for apoptosis. Indeed, apoptosis was prevented or at least significantly delayed when active nuclear import was inhibited (Shi and Skeath, 2004; Yasuhara et al., 1997). In addition, the number of proteins that undergo regulated nucleocytoplasmic transport during apoptosis is steadily growing (reviewed in Fahrenkrog, 2006; Ferrando-May, 2005). However, the transport machinery is also affected during apoptosis. For example, a nucleocytoplasmic redistribution of importin $\alpha$ and importin $\beta$ and the collapse of the RanGTP gradient was observed after induction of apoptosis (Ferrando-May et al., 2001; Miyamoto et al., 2004). These events precede caspase activation and are accompanied by an increase in NPC permeability (Ferrando-May et al., 2001; Kodiha et al., 2004). In the final stage of apoptosis, also called execution phase, a subset of nucleoporins is cleaved by caspases which probably impairs the permeability barrier of the NPC (Fahrenkrog, 2006; Ferrando-May, 2005; Patre et al., 2006). Whether caspases themselves are actively transported into the nucleus is still under discussion (Ferrando-May, 2005). Subcellular fractionation after induction of apoptosis led to the identification of active caspase-2, $-3,-6$ and -8 in the nuclear fraction (Benchoua et al., 2002; Mandal et al., 1999; Zhivotovsky et al., 1999). In another study, the subcellular localization of the caspase zymogens was analyzed through transfection of cells with GFP-fused procaspases (Shikama et al., 2001). Among them procaspase-1, -3, -6, -7 and -9 showed mainly cytoplasmic localization with different levels of nuclear localization dependent on the cell type. In contrast, procaspase-2 was exclusively nuclear while procaspase- 8 and -10 were restricted to the cytoplasm. In a more recent report, BesnaultMascard et al. (2005) demonstrated that procaspase- 8 can be sumoylated which leads to a nuclear localization. Unsumoylated procaspase-8, however, remains cytoplasmic. Until now, a NLS was only identified in the prodomain of procaspase-2 (Colussi et al., 1998) and the nuclear localization of this procaspase is widely accepted. With regard to other caspases, the necessity of active nuclear transport is still questioned (Ferrando-May, 2005). Since the increase in NPC permeability precedes caspase activation they might alternatively enter the nucleus by passive diffusion. In addition, nucleoporins belong to the substrates of executioner caspases, therefore, these downstream caspases simply might "cleave their way" into the nucleus. 


\subsection{The aim of this study}

The function of the apoptotic nuclease DFF40 is clearly nuclear and it was suggested that DFF40 is transported into the nucleus complexed with its inhibitor DFF45 (Lechardeur et al., 2000; Liu et al., 1998; Samejima and Earnshaw, 1998). However, despite reports on the identification of NLSs within the C-termini of both DFF subunits (Lechardeur et al., 2000; Samejima and Earnshaw, 2000) the exact mechanism of nuclear transport of DFF has not been elucidated so far. Therefore, the primary goal of this study was to determine the nuclear transport pathway of the DFF complex. More detailed, by in vivo transfection experiments with fluorescently tagged DFF subunits amino acid residues essential for nuclear accumulation should be identified. In vitro binding studies with bacterially expressed DFF complexes were applied to identify potential nuclear import receptors. Subsequently, the functional relevance of the transport factor-DFF interaction should be confirmed by in vitro nuclear import assays with digitioninpermeabilized cells. These different approaches were also used to examine whether monomeric DFF subunits are independently recognized and translocated by importins. 


\section{Results}

Apoptosis is often accompanied by digestion of genomic DNA. The major cellular cleavage tool is the heterotetrameric DNA fragmentation factor (DFF) that consists of an inhibitory subunit (DFF45) and a catalytic subunit (DFF40). Although the intracellular function of DFF40 is clearly nuclear, the subcellular localization of the DFF complex has been a matter of debate for a long time. Initially, the DFF complex was isolated from cytosolic fractions of mouse lymphoma cells (Enari et al., 1998; Sakahira et al., 1998). These results led to the assumption that active DFF40 is translocated into the nucleus after caspase-3 mediated cleavage of DFF45 in the cytoplasm. In contrast, localization studies of other groups clearly showed that the DFF subunits are localized in the nuclear compartment in healthy cells (Lechardeur et al., 2000; Liu et al., 1998; Samejima and Earnshaw, 1998). Thus, the current view is that the DFF complex is transported into the nucleus where DFF40 is activated after induction of apoptosis. Putative classical NLSs (cNLS) were predicted from the amino acid sequences of the C-terminal regions of DFF40 and DFF45 and it was shown that both C-termini are involved in the nuclear translocation of DFF (Enari et al., 1998; Lechardeur et al., 2000; Samejima and Earnshaw, 2000). However, the nuclear transport mechanism has not been characterized so far. To analyze the processes underlying the nuclear import of the DFF complex, we first asked whether the DFF complex traverses the nuclear membrane in a transport receptor-mediated manner.

\subsection{Nuclear import of the DFF complex is energy-dependent and requires access to nuclear pore proteins}

The nuclear uptake of the DFF40/DFF45 complex was examined using in vitro nuclear import assays (Adam et al., 1990). This method allows the reconstitution of nuclear import processes in the absence of endogenous cytoplasmic components. For that purpose, HeLa cells were grown on glass coverslips and the plasma membranes were selectively permeabilized with digitonin. Nuclear import of the cargo of interest can be reconstituted using either rabbit reticulocyte lysate as a source of import receptors or purified recombinant import receptors and further components of the transport system. To visualize the nuclear import of the DFF complex directly, we generated recombinant fluorescently labeled DFF by fusing EGFP to the N-terminus of DFF40. For purification of the complex DFF45 was N-terminally His-tagged. Since DFF45 needs to be present for correct folding and activity of DFF40 both subunits were coexpressed in E. coli and subsequently the complex was affinity purified on Ni-NTA-agarose. The EGFP- and His-tag were fused in close proximity to the CIDE domains of DFF40 and DFF45, respectively, which are involved in the dimerization of the subunits. As a functional control, a plasmid DNA 
cleavage assay was performed to examine whether the tags interfere with proper folding of the subunits and complex formation (Fig. 6A). In this cleavage assay purified DFF40/DFF45 complex was incubated with recombinant active caspase-3 and the activation of DFF40 was monitored by the cleavage of plasmid DNA. The plasmid DNA was efficiently cleaved upon incubation with EGFP-DFF40/His-DFF45 and purified caspase-3 (Fig. 6A, lanes 3-7). This demonstrates that the EGFP-DFF40/His-DFF45 complex can be activated by caspase-3 leading to the degradation of DFF45 and release of nucleolytically active EGFP-DFF40. In contrast, the plasmid DNA was not cleaved in the presence of caspase-3 or epitope-tagged DFF alone (Fig. $6 A$, lanes 2 and 8). For technical reasons, we had to clone His-tagged DFF45 lacking the first seven amino acid residues. The DNA cleavage assay showed that the deletion of amino acids 1-7 in DFF45 did not interfere with its dual function as chaperone and inhibitor of DFF40.

A

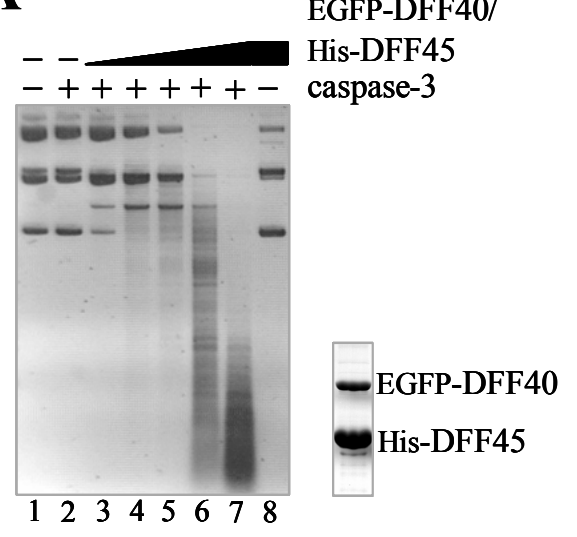

B

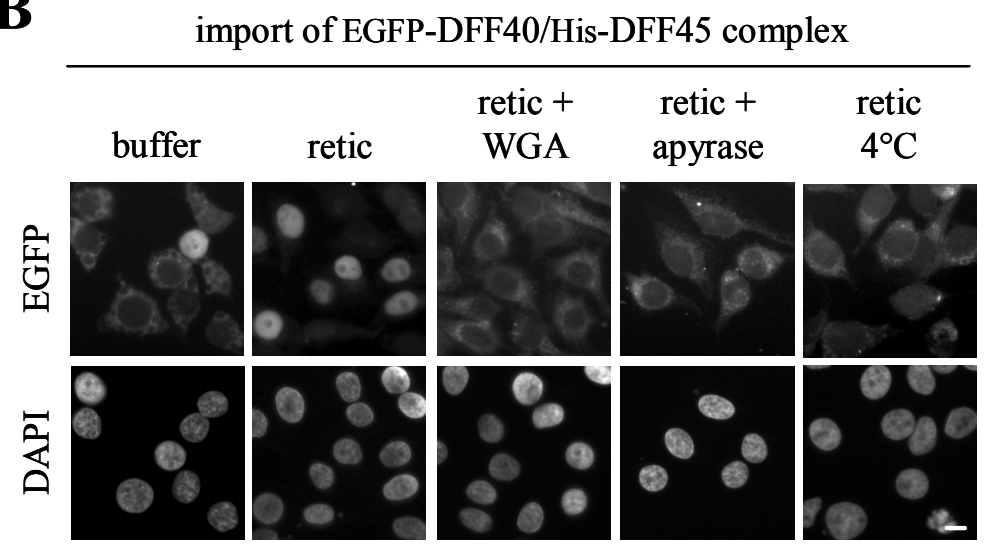

FIG. 6: The DFF complex is actively imported into the nucleus. $A$, plasmid assay for analyzing DFF40 activity. The EGFP-DFF40/His-DFF45 complex can be activated by caspase-3 leading to the release of nucleolytically active EGFP-DFF40. Plasmid DNA was incubated with increasing amounts of EGFP-DFF40/HisDFF45 (lane 3: $18 \mathrm{nM}$, lane 4: $45 \mathrm{nM}$, lane 5: $90 \mathrm{nM}$, lane 6: $180 \mathrm{nM}$, lane 7 and 8: $360 \mathrm{nM}$ ) in the presence or absence of caspase-3 for $2 \mathrm{~h}$ at $37^{\circ} \mathrm{C}$. Neither caspase-3 (lane 2) nor epitope-tagged DFF complex (lane 8) alone were able to cleave plasmid DNA. After phenol-chloroform extraction, the DNA was analyzed on a $1 \%$ agarose gel (left panel). The bands in lane 1 (plasmid control) represent the three topological forms of circular plasmid DNA. A Coomassie-stained SDS-PAGE gel of purified EGFP-DFF40/His-DFF45 complex used for the DNA cleavage assay is shown on the right of the agarose gel. $B$, nuclear transport of the EGFP-DFF40/His-DFF45 complex is energydependent and requires access to nucleoporins. Digitonin-permeabilized HeLa cells were incubated with $0.35 \mu \mathrm{M}$ substrate, rabbit reticulocyte lysate (retic) and an energy-regenerating system for $25 \mathrm{~min}$ at $37^{\circ} \mathrm{C}$ (for details see Materials and Methods). For a negative control, retic was replaced by transport buffer (buffer). Import reactions under inhibitory conditions were carried out either after pretreating the cells with wheat germ agglutinin (WGA), by replacing the energy-regenerating system with apyrase or by doing the assay at $4^{\circ} \mathrm{C}$ as indicated. Cells were fixed and studied by direct fluorescence (EGFP). The DNA was counterstained with DAPI. EGFP-DFF40/His-DFF45 tended to slightly diffuse into nuclei in the absence of import receptors (buffer). Scale bar represents $10 \mu \mathrm{m}$. 
The purified EGFP-DFF40/His-DFF45 complex was used in the in vitro nuclear import assay (Fig. 6B). The permeabilized HeLa cells were incubated with EGFP-DFF40/His-DFF45, reticulocyte lysate and an energy-regenerating system (for more details, see also Materials and Methods). Although EGFP-DFF40/His-DFF45 was mainly restricted to the cytoplasm in the absence of import receptors (buffer), nuclear accumulation could already be observed in some cells. However, the addition of reticulocyte lysate strongly enhanced the nuclear uptake leading to a complete nuclear staining of the EGFP-DFF40/His-DFF45 complex. This indicates that the nuclear transport of DFF is import receptor-dependent.

To further characterize the transport mechanism of the DFF complex, in vitro nuclear import assays were carried out under inhibitory conditions (Fig. 6B). On one hand the cells were pretreated with wheat germ agglutinin (WGA). On the other hand the energy-regenerating system was replaced with apyrase or the assay was performed at $4{ }^{\circ} \mathrm{C}$, respectively (Fig. $6 B$ ). Application of these different transport inhibitors strongly blocked the nuclear accumulation of the EGFP-DFF40/His-DFF45 complex. WGA binds to $\mathrm{N}$-acetylglucosylated nucleoporins, thereby inhibiting receptor-mediated transport processes through the nuclear pore complexes while passive diffusion remains unaffected (Finlay et al., 1987; Yoneda et al., 1987). Hence, nuclear import of DFF requires access to nucleoporins. In addition, the loss of nuclear uptake at low temperature $\left(4^{\circ} \mathrm{C}\right)$ and after ATP depletion by apyrase treatment underlines the energydependence of the nuclear transport and excludes passive diffusion. Taken together, these results demonstrate that DFF traverses the nuclear envelope in a receptor-mediated fashion as the majority of nuclear proteins.

\subsection{The importin $\alpha / \beta$ heterodimer specifically binds to the DFF complex}

To identify potential nuclear transport receptors that mediate the nuclear import of DFF, in vitro binding studies using GST-pull down assays were performed. GST-tagged DFF40 and Histagged DFF45 were coexpressed in E. coli and tandem affinity purified. More detailed, to yield a DFF complex with equimolar concentrations of both subunits, the GST-DFF40/His-DFF45 complex was first purified on Ni-NTA agarose and subsequently on glutathione-Sepharose. The proper folding of the subunits and complex formation was again analyzed in a plasmid assay (Fig. 7A). Upon activation of the purified GST-DFF40/His-DFF45 complex by caspase-3, GSTDFF40 was able to cleave plasmid DNA (Fig. 7A, lane 3 and 4), demonstrating that the GSTDFF40/His-DFF45 complex is functional. 

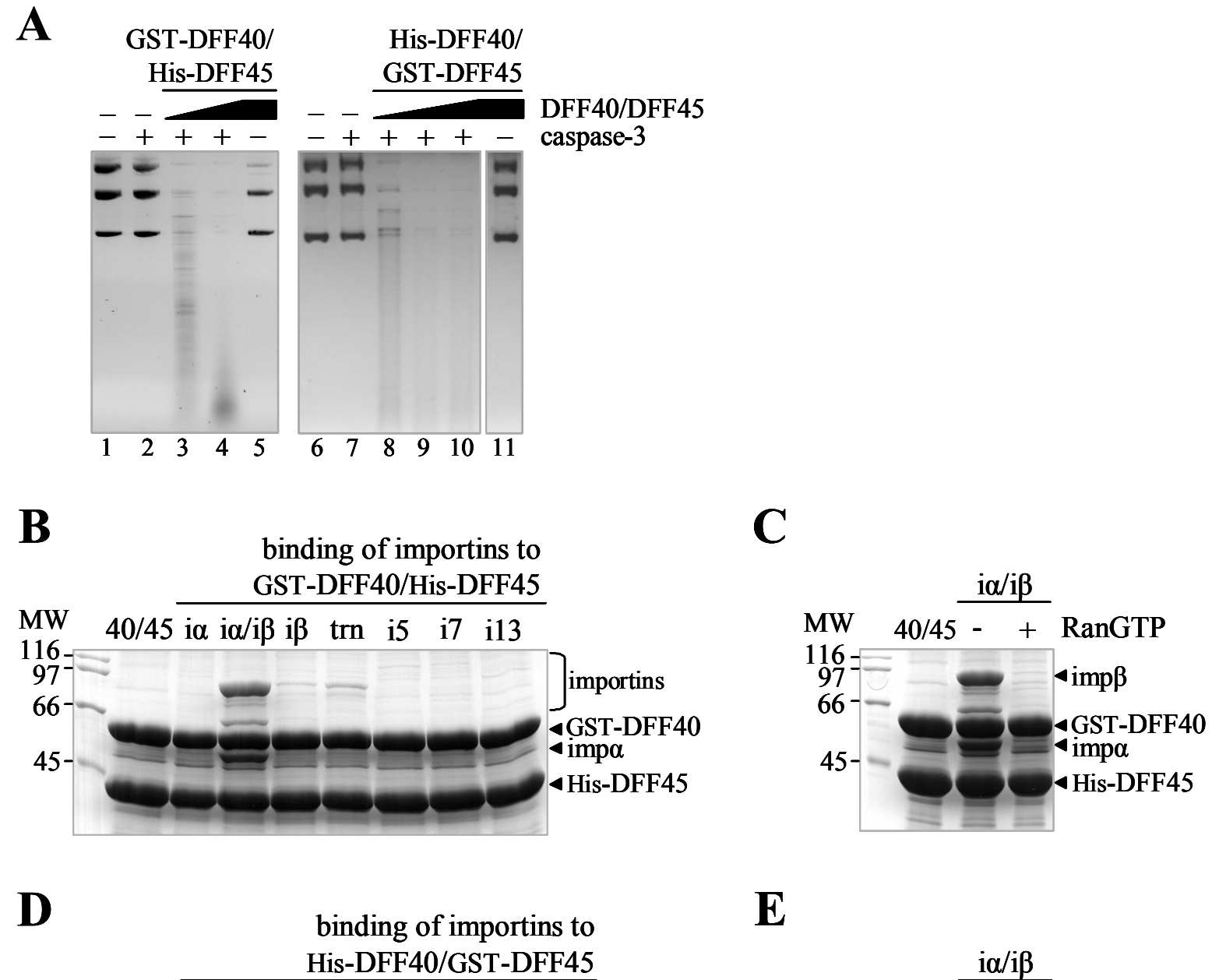

$\mathbf{E}$
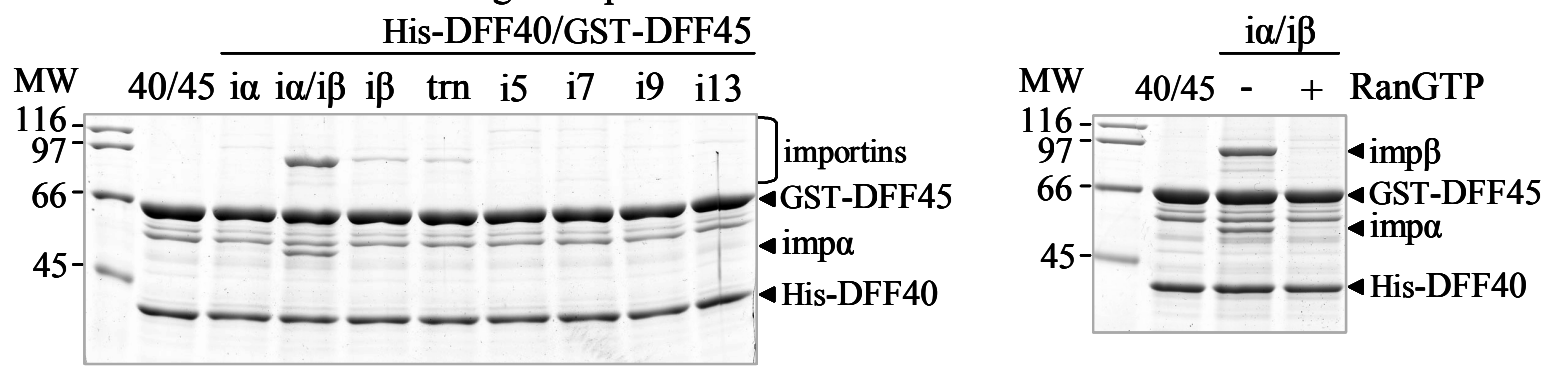

FIG. 7: The DFF40/DFF45 complex specifically interacts with the importin $\alpha / \boldsymbol{\beta}$ heterodimer. $A$, plasmid assay for analyzing DFF40 activity. The GST-DFF40/His-DFF45 complex and the His-DFF40/GST-DFF45 complex can be activated by caspase-3 leading to the release of nucleolytically active GST-DFF40 or His-DFF40, respectively. Plasmid DNA was incubated with increasing amounts of GST-DFF40/His-DFF45 (lane 3-5) or HisDFF40/GST-DFF45 (lane 8-11) in the presence or absence of caspase-3 for $2 \mathrm{~h}$ at $37^{\circ} \mathrm{C}$. Neither caspase-3 (lane 2 and 7) nor epitope-tagged DFF complex (lane 5 and 11) alone were able to cleave plasmid DNA. After phenolchloroform extraction, the DNA was analyzed on a 1\% agarose gel. The bands in lane 1 and 3 (plasmid control) represent the three topological forms of circular plasmid DNA. $B$ and $D$, interactions of DFF with different import receptors were analyzed in GST-pull down assays. Epitope-tagged DFF40 and DFF45 were coexpressed in E. coli and the DFF40/DFF45 complex (40/45) was immobilized on glutathione-Sepharose. The GST-DFF40/His-DFF45 complex $(B)$ or the His-DFF40/GST-DFF45 complex $(D)$ was incubated with importin $\alpha$, importin $\alpha / \beta$, importin $\beta$, transportin (trn), importin 5, importin 7, importin 9 or importin 13, all from bacterial lysates. (continued on page 24) 
For the in vitro binding studies GST-DFF40/His-DFF45 was immobilized on glutathioneSepharose and incubated with importin $\alpha$, importin $\alpha / \beta$ heterodimer, importin $\beta$, transportin, importin 5, importin 7 and importin 13, all from bacterial lysates (Fig. 7B). After washing the beads, bound proteins were analyzed by SDS-PAGE followed by Coomassie staining. Only the importin $\alpha / \beta$ heterodimer was bound efficiently to the DFF comples. The binding of importin $\beta$ and transportin was very weak and none of the other import receptors was bound (Fig. 7B).

To examine whether the binding of importin $\alpha / \beta$ to the GST-DFF40/His-DFF45 complex is specific, GST-pull down assays were carried out in the presence of RanGTP (Fig. 7C). While import receptors bind their cargoes in the cytoplasm in the absence of RanGTP, direct binding of RanGTP to $\beta$-family import receptors disintegrates the receptor-cargo interaction in the nucleus (Görlich et al., 1996b; Izaurralde et al., 1997). Thus, the addition of RanGTP in the GST-pull down assays should inhibit specific import receptor-cargo interactions. Immobilized GSTDFF40/His-DFF45 was incubated with importin $\alpha / \beta$ in the absence or presence of RanGTP and the bound proteins were analyzed by SDS-PAGE followed by Coomassie staining (Fig. 7C). Indeed, the binding of importin $\alpha / \beta$ to DFF was abolished in the presence of RanGTP suggesting a functional relevance.

As mentioned above, the DFF subunits were affinity-tagged for protein purification and in vitro interaction studies. To exclude that these tags interfere with import receptor binding or influence the interactions between import receptors and GST-DFF40/His-DFF45, a His-DFF40/GSTDFF45 complex was generated. In addition, His-DFF40-mediated cleavage of plasmid DNA upon incubation of His-DFF40/GST-DFF45 with purified caspase-3 revealed that this complex is functional as well (Fig. 7A, lane 8-10). Immobilized His-DFF40/GST-DFF45 was incubated with importin $\alpha$, importin $\alpha / \beta$, importin $\beta$, transportin, importin 5 , importin 7 , importin 9 and importin 13, all from bacterial lysates. Bound proteins were analyzed by SDS-PAGE followed by Coomassie staining. As it was the case for the GST-DFF40/His-DFF45 complex, only importin $\alpha / \beta$ was efficiently and specifically bound to His-DFF40/GST-DFF45 and this binding was abolished by RanGTP (Fig. $7 D$ and $E$ ). Importin $\beta$ and transportin showed a very weak binding and none of the other import receptors was bound (Fig. 7D).

\section{FIG. 7: continued}

Bound fractions were analyzed by SDS-PAGE followed by Coomassie staining. Only the importin $\alpha / \beta$ heterodimer bound efficiently to the DFF40/DFF45 complex, while the binding of importin $\beta$ and transportin was very low. $C$ and $E$, GST-pull down assays in the absence and presence of RanGTP were performed as described in $B$ and $D$. The binding of importin $\alpha / \beta$ to GST-DFF40/His-DFF45 (C) or His-DFF40/GST-DFF45 (E) was abolished by RanGTP $(2 \mu \mathrm{M})$, which was used to simulate nuclear conditions. MW, molecular weight in kilodalton; imp (i), importin. 


\subsection{Specific binding of different importin $\alpha$ isoforms to the DFF complex}

In contrast to the members of the $\beta$-family transport receptors multiple subtypes of the importin $\alpha$ adapter exist in eukaryotes. More specifically, six different importin $\alpha$ isoforms have been identified in humans (see also Introduction). Importin $\alpha$ proteins show a high degree of conservation between higher and lower eukaryotes which is reflected by the essentially identical structures of the NLS binding domains of yeast and mouse importin $\alpha$ (reviewed in Conti, 2002). In the in vitro interaction studies described in the previous section Xenopus leavis $(X$.) importin $\alpha 1$ was used instead of human importin $\alpha$ (not in hands). $X$. importin $\alpha 1$, a homologue of human importin $\alpha 2$ (Fig. $8 A$ ), was the importin $\alpha$ protein that was initially isolated (Görlich et al., 1994) and therefore, has been used in many studies on nuclear transport. $X$. importin $\alpha 1$ bound very efficiently to the DFF complex (Fig. $7 B$ ). To test whether the DFF complex is preferentially bound by $X$. importin $\alpha 1$ or if other $X$. importin $\alpha$ subtypes are even more functional regarding the interaction with DFF, GST-pull down assays with different Xenopus importin $\alpha$ isoforms were performed (Fig. 8). For that purpose, untagged and myc-tagged importin $\alpha$ subtypes were transcribed and translated in vitro with the $\mathrm{T}_{\mathrm{N}} \mathrm{T}$ coupled reticulocyte lysate system (for details, see Material and Methods). Immobilized GST-DFF40/His-DFF45 complex was preincubated with 10\% BSA to reduce unspecific binding of the import receptors. Subsequently, the DFF complex was incubated with six ${ }^{35}$ S-labeled Xenopus importin $\alpha$ isoforms together with recombinant purified importin $\beta$. An aliquot of the in vitro transcribed and translated $\left(\mathrm{T}_{\mathrm{N}} \mathrm{T}\right)$ Xenopus importin $\alpha$ subtypes (Fig. $8 \mathrm{C}$ ) and the bound fractions (Fig.8D) were analyzed by SDS-PAGE followed by phosphorimaging. The GST-DFF40/His-DFF45 complex was strongly bound by Xenopus importin $\alpha 1$ and $\alpha 2$ and moderately bound by Xenopus importin a5.1. The reduced binding in the presence of RanGTP points to a specific interaction (Fig. 8D). While Xenopus importin $\alpha 1$ and $\alpha 2$, both being homologues of human importin $\alpha 2$, belong to the Rch1 subclass, Xenopus importin $\alpha 5.1$ represents a NPI-1 subtype. Xenopus importin $\alpha 3, \alpha 4$ and a5.2 exhibited only weak binding to GST-DFF40/His-DFF45 and this binding was RanGTP insensitive. Among the variants that were specifically bound to the DFF complex Xenopus importin $\alpha 1$ showed the highest binding competence (37\%, Fig. 8D) and therefore was used in further studies. Generally, it can be concluded that the DFF complex preferably interacts with importin $\alpha$ adapters belonging to the Rch1 subclass. 


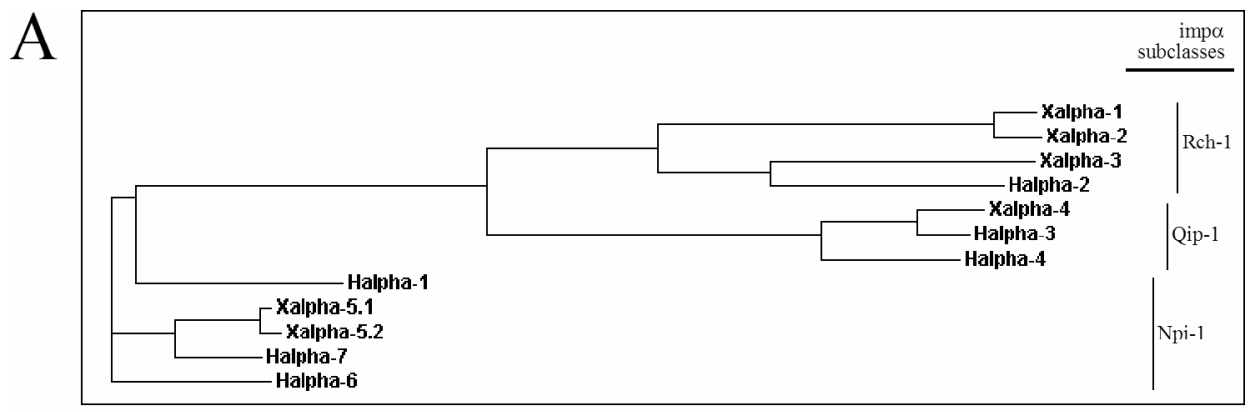

$\mathrm{B}$

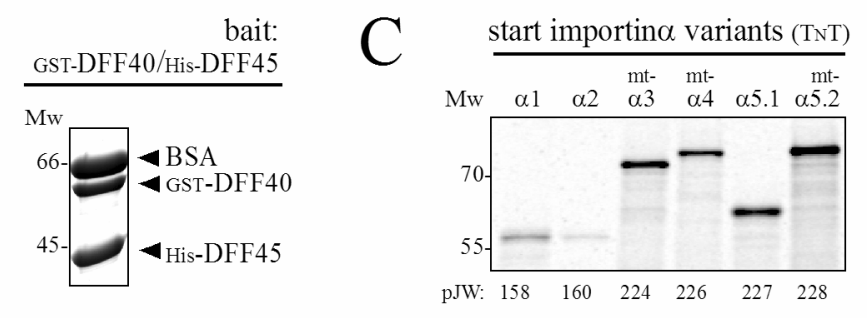

$\mathrm{D}$

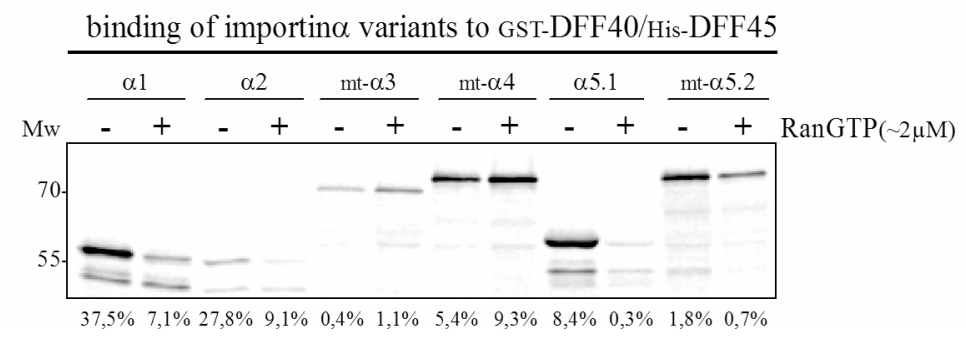

FIG. 8: Specific binding of different importin $\alpha$ isoforms to the DFF complex. $A$, phylogenetic tree of human $(\mathrm{H})$ and Xenopus laevis $(\mathrm{X})$ importin $\alpha$ isoforms is shown in panel $A$. The GenBank ${ }^{\mathrm{TM}}$ accession numbers are as follows: Halpha-1 (P52294), Halpha-2 (P52292), Halpha-3 (O00505), Halpha-4 (O00629), Halpha-6 (O15131), Halpha-7 (O60684), Xalpha-1 (P52170), Xalpha-2 (P52171), Xalpha-3 (Q7ZX00), Xalpha-4 (Q70PC6), Xalpha-5.1 (Q70PC5), and Xalpha-5.2 (Q70PC4). B, the two DFF-subunits, GST-DFF40 and His-DFF45, were expressed in $E$. coli and used as bait after immobilization on glutathione-Sepharose. $C$ and $D$, the immobilized GST-DFF40/HisDFF45-complex was incubated with six ${ }^{35} \mathrm{~S}$-labeled Xenopus laevis importin $\alpha$ isoforms, all from the $\mathrm{T}_{\mathrm{N}} \mathrm{T}$ coupled reticulocyte lysate, together with recombinant purified importin $\beta(0.2 \mu \mathrm{M})$. Starting material (20\% of the importin $\alpha$ subtypes that were used; see panel $C$ ) and bound fractions were analyzed by SDS-PAGE followed by phosphorimaging (Amersham). As shown in panel D, importin $\alpha 1$, importin $\alpha 2$, and importin $\alpha 5.1$ were bound specifically to the immobilized GST-DFF40/His-DFF45-complex and this binding was reduced by RanGTP $(2 \mu \mathrm{M})$. Among the importin $\alpha$ isoforms used for the importin $\alpha / \beta$ binding to the DFF-complex, importin $\alpha 1$ showed the highest binding competence (see relative binding in percent written below the gels, quantified with the program ImageQuant 5.2). MW, molecular weight in kilodalton; imp, importin; mt, myc-tagged. 


\subsection{The importin $\alpha / \beta$ heterodimer is responsible for nuclear transport of the DFF complex}

Out of the tested import receptors, only importin $\alpha / \beta$ was efficiently bound to the DFF complex (Fig. 7). To examine whether the importin $\alpha / \beta$ heterodimer is able to mediate nuclear transport of DFF, we reconstituted nuclear import in vitro by using purified recombinant importin $\alpha$ and importin $\beta$ (Fig. 9A). Permeabilized HeLa cells were incubated with EGFP-DFF40/His-DFF45, purified importin $\alpha$ and importin $\beta$, a Ran mix (for details, see Materials and Methods) and an energy-regenerating system. The EGFP-DFF40/His-DFF45 complex was carried into the nuclei of permeabilized HeLa cells in the presence of importin $\alpha / \beta$ although the nuclear uptake was not

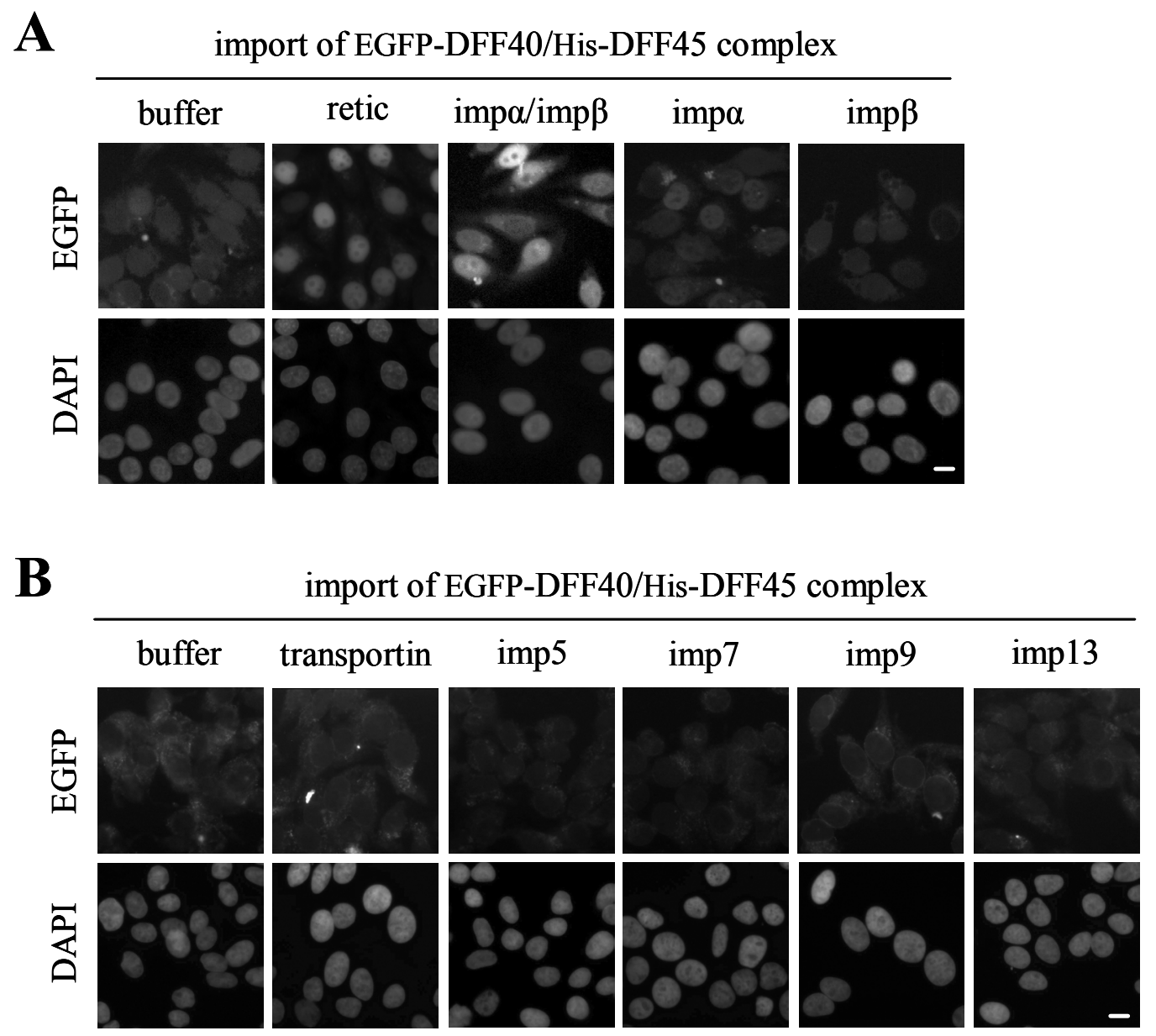

FIG. 9: Nuclear transport of DFF is mediated by the importin $\boldsymbol{\alpha} / \boldsymbol{\beta}$ heterodimer. $A$ and $B$, import assays were performed as described in the legend to Fig. 6. Permeabilized HeLa cells were incubated with reticulocyte lysate (retic) or the indicated import receptors $(0.3 \mu \mathrm{M})$, a Ran mix (see Materials and Methods) and an energyregenerating system for $25 \mathrm{~min}$ at $37^{\circ} \mathrm{C}$. EGFP-DFF40/His-DFF45 was imported into the nucleus only in the presence of reticulocyte lysate and importin $\alpha / \beta$. However, weak nuclear import of EGFP-DFF40/His-DFF45 could also be observed adding importin $\alpha$ alone. Scale bar represents $10 \mu \mathrm{m}$. imp, importin. 
as efficient as with reticulocyte lysate. Since DFF can be recognized by different importin $\alpha$ isoforms (see again Fig. $8 D$ ) the better nuclear uptake in the presence of reticulocyte lysate may be explained by the broader range of suitable importin $\alpha$ molecules. In addition, weak nuclear import of EGFP-DFF40/His-DFF45 was also observed when only importin $\alpha$ was added (Fig. $9 A$ ). That is probably due to the formation of an $\alpha / \beta$-heterodimer of the added importin $\alpha$ with not completely washed out endogenous importin $\beta$. To remove the soluble cytoplasmic components the permeabilized HeLa cells were extensively washed. However, because of its interaction with nucleoporins a low concentration of importin $\beta$ remained in the cells. Thus, basal nuclear import can be observed after the addition of importin $\alpha$ in the absence of exogenous importin $\beta$ (Görlich et al., 1995b).

In vitro nuclear import assays were also carried out with import receptors that did not efficiently bind to the DFF complex in the GST-pull down assays. Importin $\beta$ (Fig. 9A), transportin, importin 5, importin 7, importin 9 and importin 13 (Fig. 9B) were not able to translocate the EGFP-DFF40/His-DFF45 complex into the nuclei of digitonin-permeabilized HeLa cells, confirming the results of the in vitro binding assays.

In conclusion, nuclear transport of the DFF complex is mediated by the importin $\alpha / \beta$ heterodimer. Next, we wanted to examine which regions or sequence elements of DFF are involved in the interaction with importin $\alpha / \beta$. More precisely, we wanted to answer the question whether both subunits contain regions that are essential for the importin $\alpha / \beta$-mediated nuclear uptake or not.

\subsection{Nuclear import of the DFF complex requires the C-terminal regions of both subunits}

For the initial characterization of the sequence elements necessary for the nuclear accumulation of DFF the subcellular localization of coexpressed, fluorescently tagged DFF subunits was examined in HeLa P4 cells. Since EGFP- or RFP-tagged DFF40 and DFF45 tend to diffuse into the nucleus (data not shown) one subunit was expressed as EGFP-GST fusion in order to further increase the size of the protein. Doing so, passive diffusion of the DFF complex was excluded.

To start, C-terminal deletions of both DFF subunits were coexpressed with the corresponding wild type subunit (Fig. 10) to test whether the two C-termini contribute to nuclear targeting of DFF. Previous studies by Lechardeur et al. (2000) indicated that. As in the case of bacterially expressed DFF complexes, we first analyzed whether the N-terminal tags interfere with DFF complex formation. For this purpose, EGFP-GST-DFF40 constructs were cotransfected with RFP-DFF45 constructs and vice versa. $24 \mathrm{~h}$ post-transfection the cells were lysed and the EGFPGST-fused proteins were precipitated with glutathione-Sepharose. Coprecipitated proteins were 
A

\begin{tabular}{|c|c|c|c|c|c|c|c|}
\hline- & & & & & & & EG-DFF40 wt \\
\hline- & & & & & & & EG-DFF40 $\Delta 324-338$ \\
\hline+ & & & & & & & R-DFF45 wt \\
\hline- & & & & & & & R-DFF45 $\Delta 306-331$ \\
\hline \begin{tabular}{l|l}
$\mathrm{i}$ & $\mathrm{b}$ \\
\end{tabular} & $\bar{i}$ & $\mathrm{~b}$ & $\mathrm{i}$ & $\mathrm{b}$ & $\mathrm{i}$ & & \\
\hline
\end{tabular}

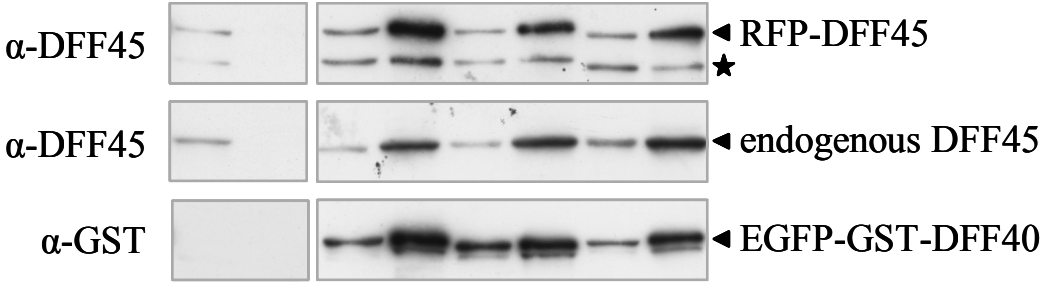

B

\begin{tabular}{|c|c|c|c|}
\hline+ & - & + & R-DFF40 wt \\
\hline- & + & - & R-DFF40 $\Delta 324-338$ \\
\hline+ & + & - & EG-DFF45 wt \\
\hline- & - & + & EG-DFF45 $\Delta 306-331$ \\
\hline b & \begin{tabular}{l|l}
$\mathrm{i}$ & $\mathrm{b}$ \\
\end{tabular} & \begin{tabular}{l|l|}
$\mathrm{i}$ & $\mathrm{b}$ \\
\end{tabular} & \\
\hline
\end{tabular}

$\alpha-R F P$

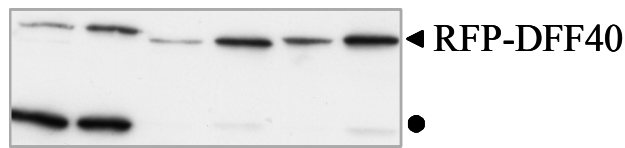

endogenous DFF45 was not detectable

$\alpha$-DFF45

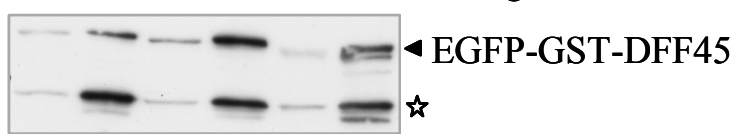

C

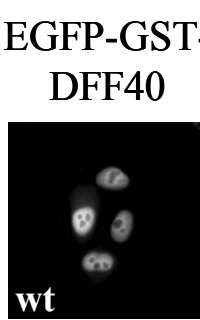

RFPDFF45

merge

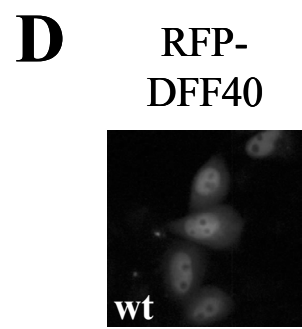

EGFP-GST-
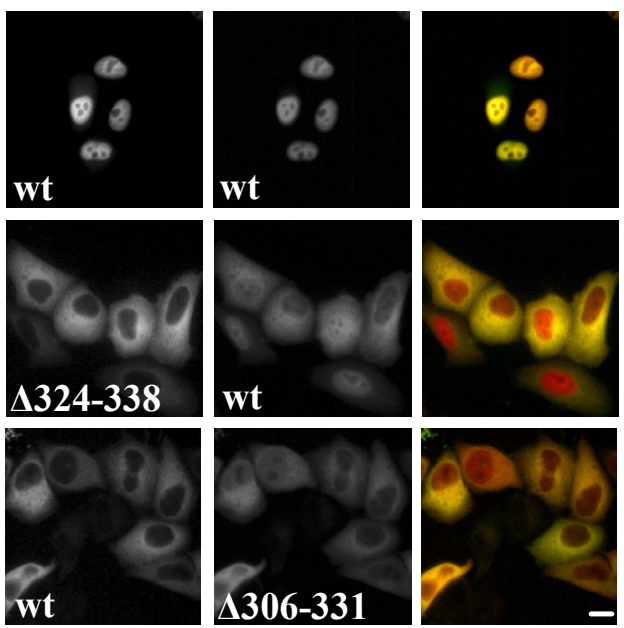

DFF45

merge
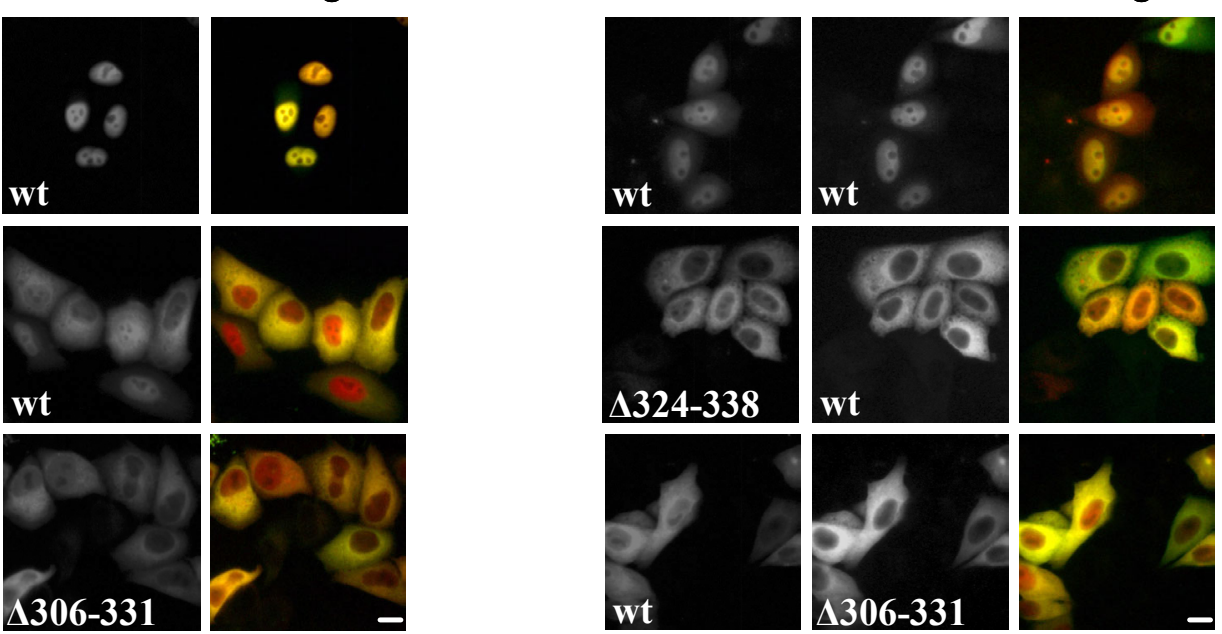

FIG. 10: The C-terminal regions of DFF40 and DFF45 are required for nuclear import of the DFF complex. Wild type and C-terminally truncated DFF subunits were fused to EGFP-GST or RFP and were cotransfected with the corresponding subunit into HeLa P4 cells. $A$ and $B$, the interaction between the DFF subunits was analyzed in GST-pull down assays $24 \mathrm{~h}$ post transfection. The cells were lysed and the (continued on page 30) 
analyzed by SDS-PAGE and subsequently immunoblotted with an anti-DFF45/35 antibody, an anti-GST antibody, and an anti-RFP antibody, respectively (Fig. 10A and $B$ ).

Wild type RFP-DFF45 coprecipitated with wild type EGFP-GST-fused DFF40 or EGFP-GSTDFF40 $\Delta 324-338$, respectively (Fig. 10A). As a control, RFP-fused DFF45 was expressed in the absence of EGFP-GST-tagged DFF40. RFP-DFF45 was not precipitated without EGFP-GSTDFF40 (Fig. 10A) demonstrating that the RFP-tagged subunits did not unspecifically interact with glutathione-Sepharose. Additionally, RFP-DFF45 $\Delta 306-331$ was coprecipitated with wild type EGFP-GST-DFF40 (Fig. 10A). Moreover, coprecipitation of DFF40 and DFF45 subunits was also observed when the tags were exchanged among the subunits (Fig. 10B). This demonstrates that the fluorescently tagged DFF subunits overexpressed in HeLa P4 cells interacted with each other. Furthermore, coimmunprecipitation revealed that RFP-DFF45 (Fig. $10 A$, indicated with an asterisk) and wild type RFP-DFF40 (Fig. 10B, indicated with a square) were specifically degraded in transfected cells These degradation fragments were also bound by EGFP-GST-DFF40 and EGFP-GST-DFF45. However, the interaction with degraded RFPDFF45 was much weaker than with full length RFP-DFF45. In contrast, the RFP-DFF40 degradation fragment was more abundant than the full length protein. EGFP-GST-DFF45 was

\section{FIG. 10: continued}

EGFP-GST-fused subunits were precipitated with glutathione-Sepharose. $1 \%$ of the lysate (input) and bound proteins were analyzed by SDS-PAGE and immunoblotted with anti-DFF45/35 polyclonal antibody (ProSci Incorporated) or anti-RFP polyclonal antibody (Molecular Biotechnology), respectively. Afterwards, the membranes were stripped to remove bound antibody and subsequently incubated with anti-GST monoclonal antibody (Santa Cruz) or anti-DFF45/35 antibody, respectively. Cotransfected subunits are indicated in the box on top of the blots. $A$, RFP-fused DFF45 subunits were coprecipitated with EGFP-GST-tagged DFF40 subunits. In addition, also endogenous DFF45 was coprecipitated. The asterisk indicates a specific RFP-DFF45 degradation fragment. $B$, RFPfused DFF40 subunits were coprecipitated with EGFP-GST-tagged DFF45 subunits. Specific degradation fragments of RFP-DFF40 (•) or EGFP-GST-DFF45 (ઐ) are indicated. EG, EGFP-GST; R, RFP; i, input; b, bound material. C and $D$, the subcellular localization of the DFF subunits was examined $24 \mathrm{~h}$ after transfection by direct fluorescence. The overlap between the green EGFP fusion protein and the red RFP fusion protein is shown in yellow (merge). Scale bars represent $10 \mu \mathrm{m}$. $C$, co-transfection of EGFP-GST-tagged DFF40 and RFP-tagged DFF45 constructs. $D$, co-transfection of RFP-tagged DFF40 and EGFP-GST-tagged DFF45 constructs. Regardless of the fused tag, wild type DFF40 and wild type DFF45 both accumulated in the nucleus of co-transfected cells. The deletion of either amino acids $324-338$ of DFF40 ( $\Delta 324-338)$ or amino acids 306-331 of DFF45 ( $\Delta 306-331)$ abolished the nuclear accumulation leading to a dominant cytoplasmic localization of both subunits. However, in all cases where deletions or mutations interfered with nuclear translocation of DFF, fluorescent staining of uncomplexed RFP-tagged DFF45 or uncomplexed RFP-tagged DFF40 was visible by red fluorescence in the nucleus. 
specifically degraded, too (Fig. 10B, indicated with an open asterisk). Additionally, the experiments showed that endogenous DFF45 coprecipitated with EGFP-GST-DFF40 besides exogenous RFP-tagged DFF45 (Fig. 10A). This however implies that a certain proportion of the formed complexes always contains native endogenous DFF45. Surprisingly, endogenous DFF45 was not included in EGFP-GST-DFF45/RFP-DFF40 complexes, which rather points to a dimeric instead of a tetrameric DFF complex (see also introduction chapter, 1.1.3.1). The integration of endogenous DFF45 in overexpressed DFF complexes probably reflects the presence of monomeric, uncomplexed DFF45 within healthy cells.

The subcellular distributions of the fluorescently tagged DFF complexes are summarized in Fig. $10 C$ and $D$. Wild type EGFP-GST-DFF40 and wild type RFP-DFF45 accumulated in the nuclei of cotransfected HeLa cells (Fig. 10C top panel). In contrast, deletion of either amino acids 324-338 of DFF40 or amino acids 306-331 of DFF45 lead to a dominant cytoplasmic colocalization of both subunits (Fig. 10C middle and bottom panel). However, red fluorescent staining of uncomplexed RFP-tagged DFF45 was also visible in the nucleus. This is probably caused by nuclear uptake of full length RFP-DFF45 and passive diffusion of C-terminally truncated RFP-DFF45 (compare Fig. 13). C-terminal truncations of the two subunits had similar effects on nuclear transport of DFF when the fluorescent tags were exchanged among the subunits (Fig. 10D). This clearly confirms the importance of both C-termini for nuclear accumulation of DFF.

\subsection{Basic amino acids in both subunits are essential for nuclear import of DFF}

To identify the amino acid sequences responsible for importin $\alpha / \beta$-mediated nuclear import of the DFF complex, we generated mutants substituting basic amino acids in the $\mathrm{C}$-terminal regions of DFF40 and DFF45 (Fig. 11A). The EGFP-GST-tagged mutant subunit was coexpressed with the corresponding RFP-tagged wild type subunit and vice versa. The results of the in vivo transfection studies with EGFP-GST-tagged mutant subunits are summarized in Fig. $11 B$ and $D$. Nuclear accumulation of the DFF40/DFF45 complex was blocked when either the basic cluster RxKRK in DFF40 (DFF40mutA, Fig. $11 B$ top panel) or the basic cluster KRxR in DFF45 (DFF45mutB, Fig. $11 D$ bottom panel) was mutated. In contrast, mutation of the basic cluster RKR of DFF40 (DFF40mutB, Fig. $11 B$ bottom panel) or substitution of the two positively charged amino acids at position 307 and 313 of DFF45 (DFF45mutA, Fig. 11D top panel) had no effect on the nuclear localization of DFF. Similar results were obtained with RFP fused mutants (Fig. $11 C$ and $E$ ) with the exception of DFF45mutA (Fig. 11E top pannel). In that case, mutation of the basic amino acids R307 and K313 in RFP-DFF45 abolished nuclear 
A

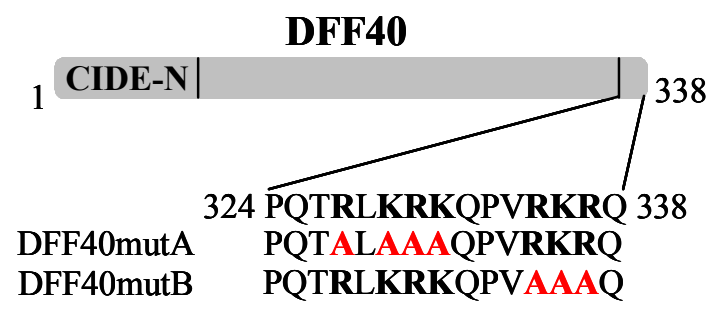

\section{DFF45}

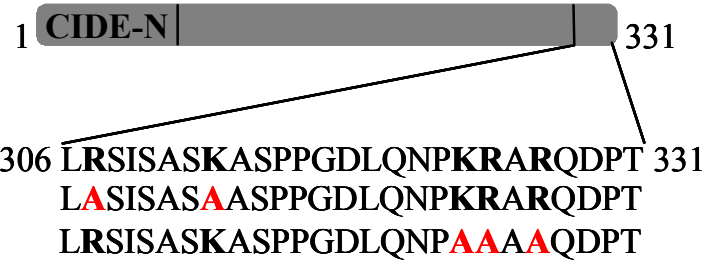

$B$ EGFP-GST- RFP-
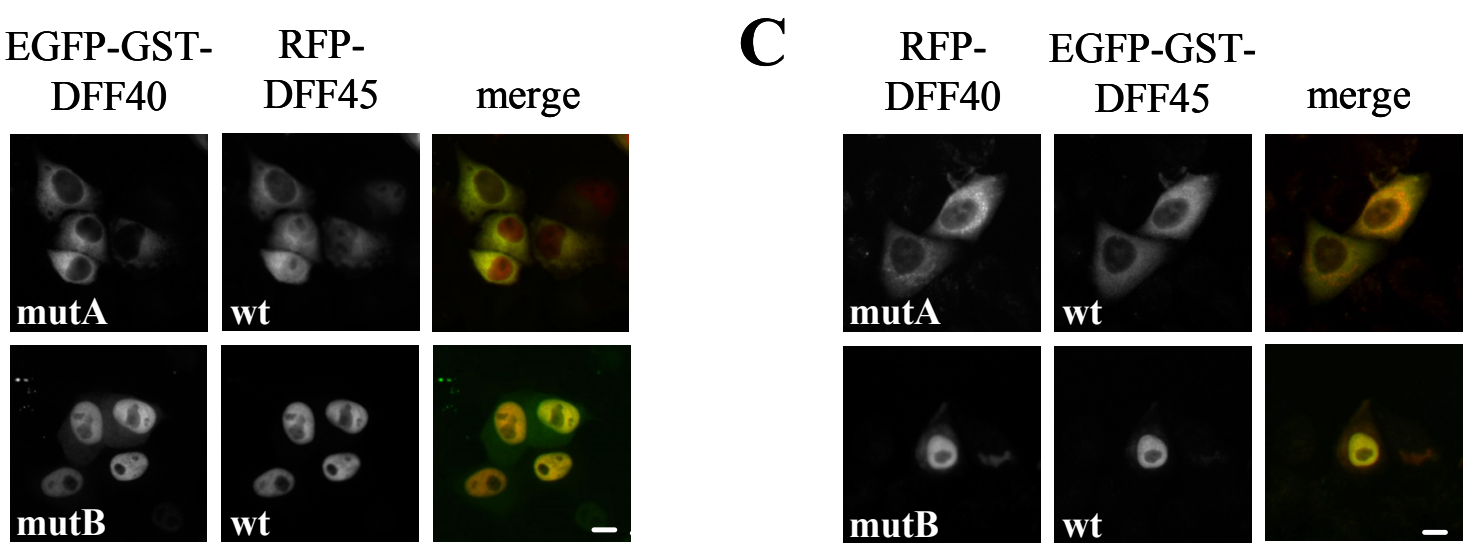

D

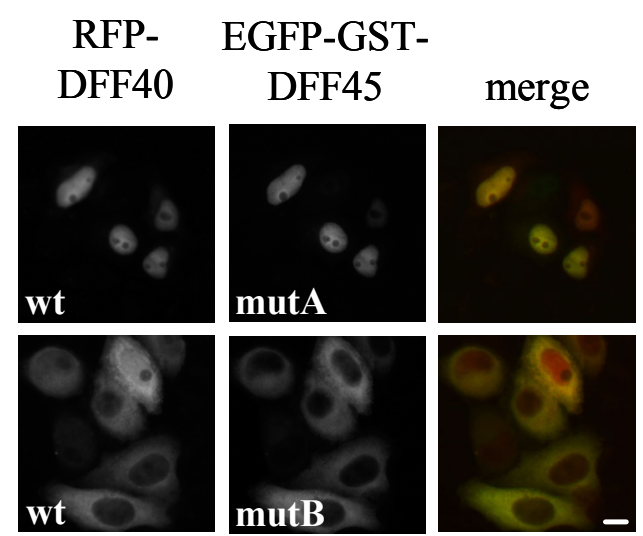

\section{E EGFP-GST- RFP-}

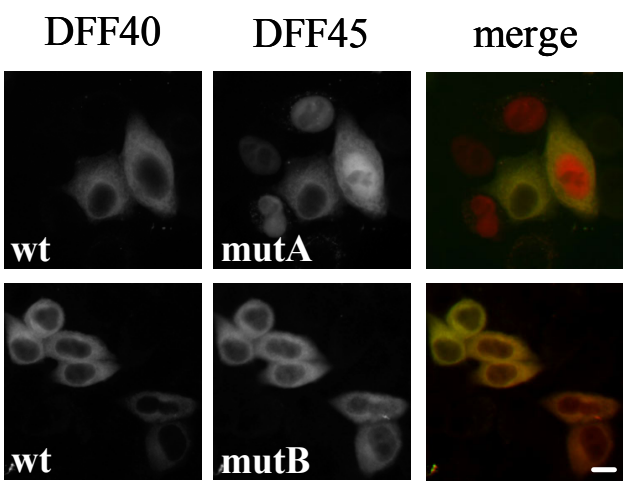

$\mathbf{F}$

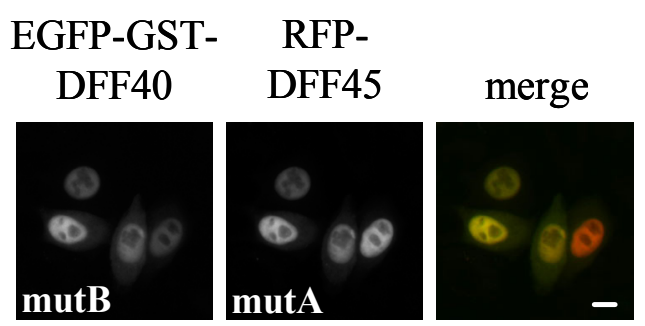

FIG. 11: Basic amino acids in the C-terminal regions of DFF40 and DFF45 are essential for nuclear accumulation of DFF. Mutated DFF subunits with alanine substitutions of basic amino (continued on page 33) 
accumulation of the EGFP-GST-DFF40/RFP-DFF45 complex. Because of the contradictory observations it remained uncertain whether the amino acids R307 and K313 are necessary for nuclear import of DFF. To resolve this discrepancy and to further elucidate the role of these basic amino acids another cotransfection experiment was performed. Here, EGFP-GSTDFF40mutB that did not affect nuclear accumulation (see Fig. $11 B$ bottom panel) was coexpressed with RFP-DFF45mutA instead of wild type EGFP-GST-DFF40. Thereby, the dominant nuclear localization pattern of the DFF40/DFF45 complex did not change (Fig. $11 F$ ), confirming that the amino acids R307 and K313 of DFF45 are not essential for nuclear translocation. However, a function of these basic amino acids in the nuclear targeting of DFF can still not be completely excluded. For that, additional experiments are indispensable. In summary, one basic cluster in the C-terminal region of each subunit, DFF40 (RxKRK) and DFF45 (KRxR), is required for nuclear import of the DFF complex. To validate this statement complex formation of transfected mutant DFF subunits was analyzed again by coprecipitation. The experiments were performed essentially as described above and the results of these subunit-interaction studies are summarized in Fig. 12. Wild type RFP-DFF45 coprecipitated with EGFP-GST-tagged DFF40 mutants (DFF40mutA and DFF40mutB; Fig. 12A) and this interaction was also observed when the tags were exchanged between the subunits (Fig. 12B). Similarly, mutant DFF45 subunits (DFF45mutA and DFF45mutB) were bound to wild type DFF40, regardless of the

\section{FIG. 11: continued}

acids in the C-terminal regions were fused to EGFP-GST or RFP and were cotransfected with the corresponding subunit into HeLa P4 cells. The subcellular localization of the subunits was examined $24 \mathrm{~h}$ after transfection by direct fluorescence. The overlap between the green EGFP-fusion protein and the red RFP-fusion protein is shown in yellow (merge). Scale bars represent $10 \mu \mathrm{m}$. A, sequences of the C-terminal regions of DFF40 (amino acids 324338) and DFF45 (amino acids 306-331). Basic amino acids are indicated by bold characters and mutated amino acids by red characters. Names of the mutant constructs are shown on the left. $B$ and $C$, mutated DFF40 subunits (DFF40mutA, DFF40mutB) fused to EGFP-GST $(B)$ or to RFP $(C)$ were cotransfected with wild type RFP-DFF45 $(B)$ or EGFP-GST-DFF45 (C). Nuclear import of DFF40 and DFF45 was blocked when the basic cluster RxKRK (DFF40mutA) was mutated (top panels): yellow fluorescence in the cytoplasm indicates cytoplasmic colocalization of the two proteins while uncomplexed RFP-DFF45 was visible by red fluorescence in the nucleus. Mutation of the basic cluster RKR (DFF40mutB) did not influence the nuclear translocation (lower panels). $D$ and E, mutated DFF45 subunits (DFF45mutA, DFF45mutB) fused to EGFP-GST $(D)$ or to RFP $(E)$ were cotransfected with wild type RFP-DFF40 $(D)$ or EGFP-GST-DFF40 $(E)$. Mutation of the basic cluster KRxR (DFF45mutB) prevented the nuclear accumulation of the DFF subunits (lower panels), while substitution of the two positively charged amino acids at position 307 and 313 in EGFP-GST-DFF45mutA did not affect nuclear transport. However, when DFF45mutA is fused to RFP nuclear accumulation of the DFF subunits was abolished. $F$, cotransfection of EGFPGST-DFF40mutB and RFP-DFF45mutA did not affect the dominant nuclear localization of the DFF complex. 


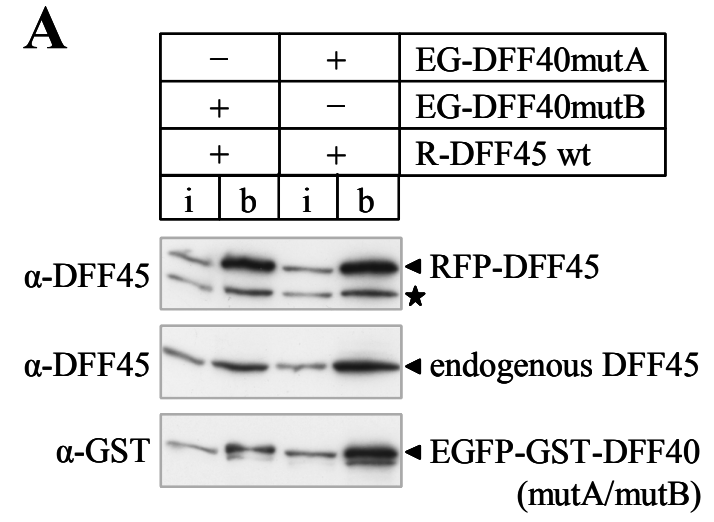

$\mathbf{C}$

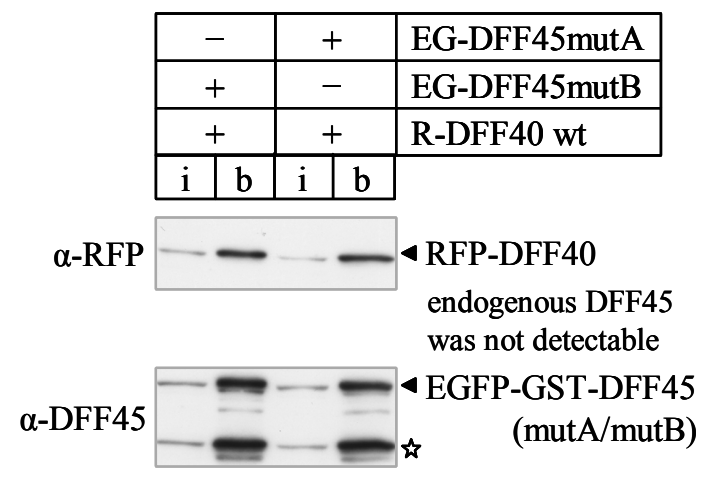

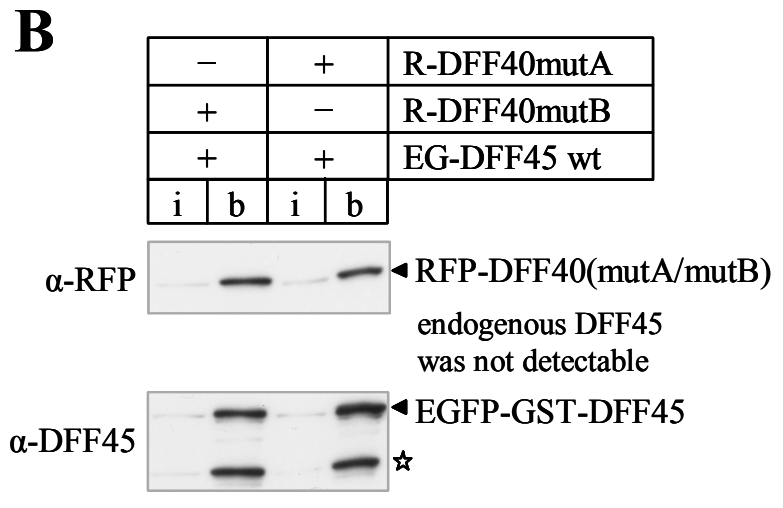

D

\begin{tabular}{|c|c|c|c|}
\hline- & + & + & R-DFF45mutA \\
\hline+ & - & - & R-DFF45mutB \\
\hline+ & + & - & EG-DFF40 wt \\
\hline- & - & + & EG-DFF40mutE \\
\hline \begin{tabular}{l|l}
$\mathrm{i}$ & $\mathrm{b}$ \\
\end{tabular} & \begin{tabular}{l|l}
$\mathrm{i}$ & $\mathrm{b}$
\end{tabular} & \begin{tabular}{l|l}
$\mathrm{i}$ & $\mathrm{b}$ \\
\end{tabular} & \\
\hline
\end{tabular}

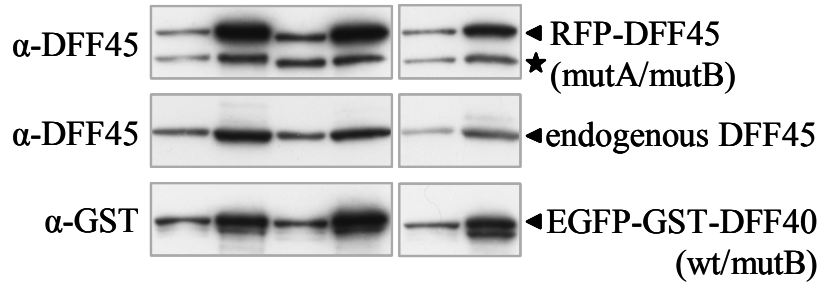

FIG. 12: Interaction of fluorescently tagged mutant DFF subunits. Mutated DFF subunits with alanine substitutions of basic amino acids in the C-terminal regions were fused to EGFP-GST or RFP and were cotransfected with the corresponding subunit into HeLa P4 cells. The interaction between the DFF subunits was analyzed in GST-pull down assays $24 \mathrm{~h}$ post transfection as described in the legend of Fig. 10. $A$ and B, mutated DFF40 subunits (DFF40mutA, DFF40mutB; see Fig. 11A) fused to EGFP-GST $(A)$ or to RFP $(B)$ were cotransfected with wild type RFP-DFF45 $(A)$ or EGFP-GST-DFF45 (B). DFF40mutA and DFF40mutB interacted with wild type DFF45 independent of the tags. In addition, endogenous DFF45 was coprecipitated with EGFP-GSTDFF45 and RFP-tagged DFF40 mutants $(A)$. $C$ and $D$, mutated DFF45 subunits (DFF45mutA, DFF45mutB) fused to EGFP-GST $(C)$ or RFP $(D)$ were cotransfected with wild type RFP-DFF40 $(C)$ or EGFP-GST-DFF40 $(D)$. Additionally, RFP-DFF45mutA was cotransfected with EGFP-GST-DFF40mutB (D). Interaction between mutant DFF45 and DFF40 subunits was observed regardless of the tags. Endogenous DFF45 was additionally included in the EGFP-GST-DFF45/RFP-DFF40 complexes $(D)$. In all cases, EGFP-GST- and RFP-tagged DFF45 subunits were specifically degraded as indicated by an open asterisk or a filled asterisk, respectively. EG, EGFP-GST; R, RFP; i, input; b, bound material.

fluorescent tags (Fig. $12 C$ and D). Additionally, RFP-DFF45mutA coprecipitated with EGFPGST-tagged DFF40mutB (Fig. 12D). The EGFP-GST-DFF40/RFP-DFF45 complexes also contained endogenous DFF45 (Fig. $12 A$ and $D$ ), as previously observed for wild type and 
truncated DFF complexes (Fig. 10). Further, EGFP-GST and RFP-tagged DFF45 subunits were again specifically degraded (Fig. 12). Taken together the coprecipitation assays clearly demonstrated that substitution of basic amino acid residues did not influence the DFF subunit interaction. Of course, this was not unexpected since the C-terminally truncated DFF subunits did not reveal interaction defects before.

So far, we showed that the DFF complex is imported into the nucleus via the classical importin $\alpha / \beta$-pathway. Amino acid sequences required for nuclear uptake were mapped to the C-termini of DFF40 and DFF45. One essential basic cluster in each of these regions was identified. Next, we took a closer look on the role of individual DFF subunits in terms of importin $\alpha / \beta$-binding and nuclear transport.

\subsection{DFF45 contains a classical NLS in its C-terminal region}

The identification of positively charged amino acid residues that are essential for the nuclear transport of the DFF complex in each subunit raised the question whether both basic clusters represent independent cNLSs. To answer this question, the nuclear transport of monomeric DFF subunits was analyzed focussing in particular on the capability of the C-terminal regions to mediate nuclear uptake. Hence, the monomeric DFF45 mutants described above (Fig. 11A), along with wild type DFF45 were expressed as EGFP-GST fusion proteins in HeLa P4 cells. While wild type DFF45 showed a clear nuclear localization, deletion of the amino acids 306-331 $(\Delta 306-331)$ or substitution of the basic cluster KRxR (mutB) blocked nuclear uptake (Fig. 13A). Mutation of R307 and K313 only had a minor effect on nuclear accumulation, leading to a homogenous distribution of monomeric DFF45 (Fig. 13A, mutA). This demonstrates that the Cterminal region, in particular the basic cluster KRxR, is essential for nuclear transport of DFF45. To analyze whether this region is also sufficient for nuclear accumulation of a cytoplasmic protein we fused it to EGFP-EGFP-GST (EEG). The exclusively cytoplasmic localization of the EGFP-EGFP-GST fusion protein changed upon fusion to amino acids 306-331 of DFF45 [EEGDFF45(aa306-331)], now being largely nuclear (Fig. 13B). These results clearly show that the Cterminal region of DFF45 exhibits a NLS that is necessary and sufficient for nuclear uptake of monomeric DFF45. In more detail, besides playing an essential role for importin $\alpha / \beta$-mediated nuclear import of the DFF complex, the basic cluster KRxR was also identified as key element of the NLS in DFF45 itself. Based on these results, it was reasonable to assume that importin $\alpha / \beta$ is also the functional import receptor for DFF45. To verify this assumption, pull down assays with immobilized GST-DFF45 and recombinant importins were performed. Importin $\alpha / \beta$ specifically bound to GST-DFF45 and this binding was abolished in the presence of RanGTP (Fig. 13C). 
A

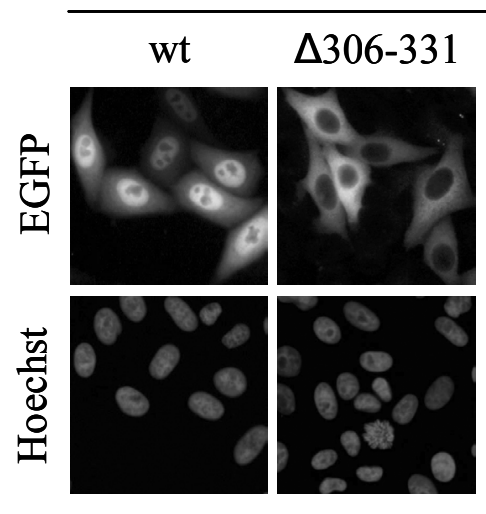

C

binding of $i \alpha / i \beta$ to GST-DFF45

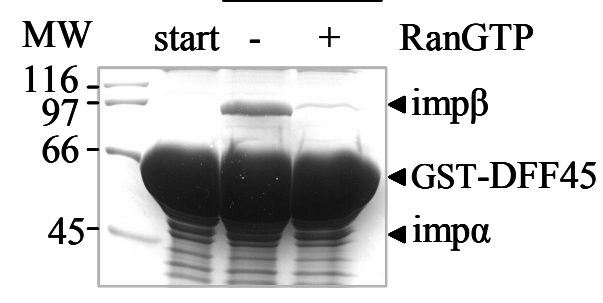

B mutB
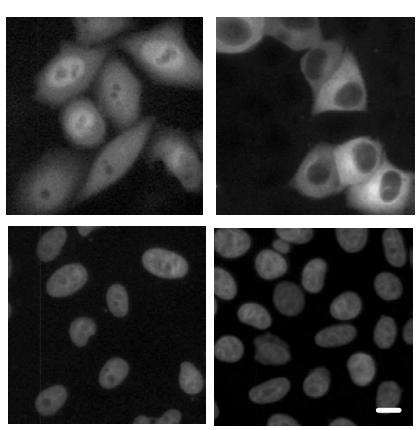

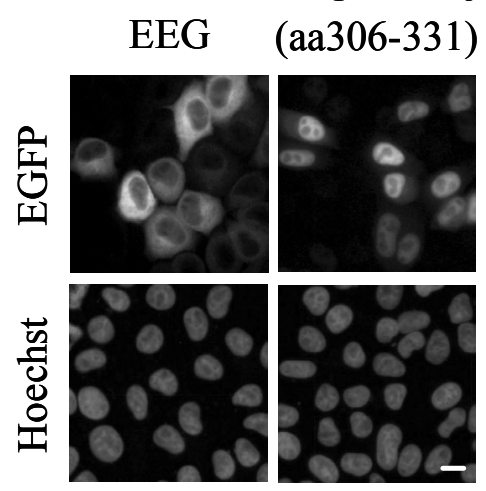

D binding of $i \alpha / i \beta$ to GST-DFF45

(aa296-331)

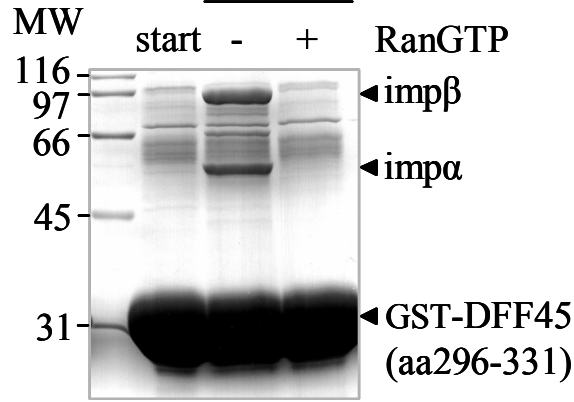

FIG. 13: The C-terminal tail of DFF45 exhibits a classical NLS that is necessary and sufficient for nuclear uptake of monomeric DFF45. $A$ and $B$, HeLa P4 cells were transiently transfected with plasmid DNA encoding wild type, truncated and mutated EGFP-GST-DFF45 $(A)$ or EGFP-EGFP-GST (EEG) and amino acids 306-331 of DFF45 fused to EEG $(B)$. The subcellular distribution was examined $24 \mathrm{~h}$ after transfection by direct fluorescence. The DNA was counterstained with Hoechst. Scale bars represent $10 \mu \mathrm{m}$. $A$, the dominant nuclear localization of wild type DFF45 was blocked when either amino acids 306-331 were deleted ( $\triangle 306-331)$ or the basic cluster KRxR was mutated (DFF45mutB, see Fig. 11A). Substitution of the two positively charged amino acids at position 307 and 313 (DFF45mutA) affected the nuclear transport only moderately. $B$, the cytoplasmic localization of EEG changed upon fusion to amino acids 306-331 of DFF45 [EEG-DFF45(aa306-331)], the localization now becoming largely nuclear. $C$ and $D$, amino acids 296-331 of DFF45 are responsible for the specific binding of importin $\alpha / \beta$ to DFF45. GST-pull down assays were performed as described in the legend to Fig. 7. Immobilized GST-DFF45 (C) and GST-fused amino acids 296-331 of DFF45 [GST-DFF45(aa296-331)] (D) were incubated with importin $\alpha / \beta$ in the absence or presence of $2 \mu \mathrm{M}$ RanGTP. Bound fractions were analyzed by SDS-PAGE followed by Coomassie staining. MW, molecular weight in kilodalton; aa, amino acids; imp (i), importin.

Furthermore, in vitro binding studies with the C-terminus of DFF45 fused to GST [GSTDFF45(aa296-331)] confirmed the specific interaction of importin $\alpha / \beta$ with this region, since importin $\alpha / \beta$ was retained (Fig. 13D). In conclusion, DFF45 harbors a classical, presumably monopartite cNLS in its C-terminal region. 
However, binding of importin $\alpha / \beta$ to DFF45 was less efficient than binding of importin $\alpha / \beta$ to the DFF complex. In other words, much more GST-DFF45 had to be immobilized to detect importin $\alpha / \beta$-binding by Coomassie staining (compare Fig. 7).

Because of the weak binding of importin $\alpha / \beta$ we analyzed whether other import receptors are involved in nuclear transport of monomeric DFF45. For that purpose, GST-pull down assays were performed with importin $\alpha$, importin $\alpha / \beta$, importin $\beta$, transportin, importin 5 , importin 7 , importin 9 and importin 13, all from bacterial lysates (Fig. 14A). Besides importin $\alpha / \beta$, none of the other import receptors interacted with GST-DFF45, strongly suggesting that DFF45 enters the nucleus via the importin $\alpha / \beta$-pathway.

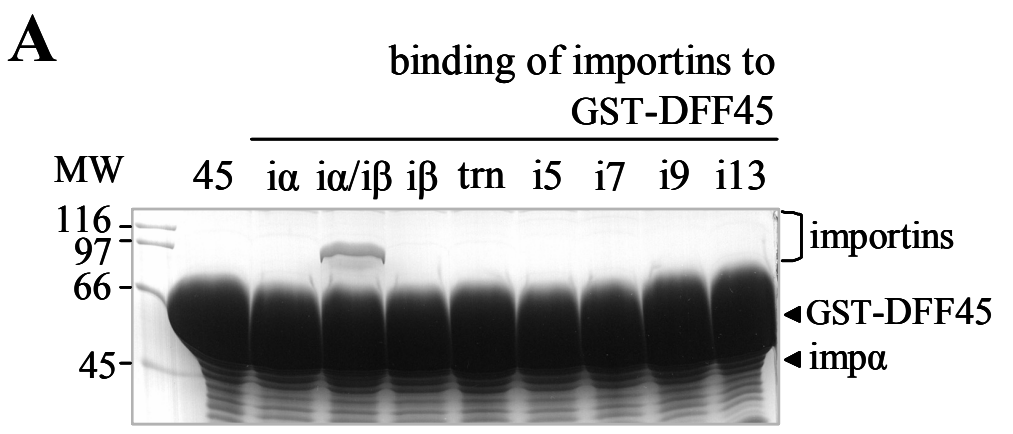

B

binding of importins to

GST-DFF40/

His-DFF45 $\Delta 306-331$

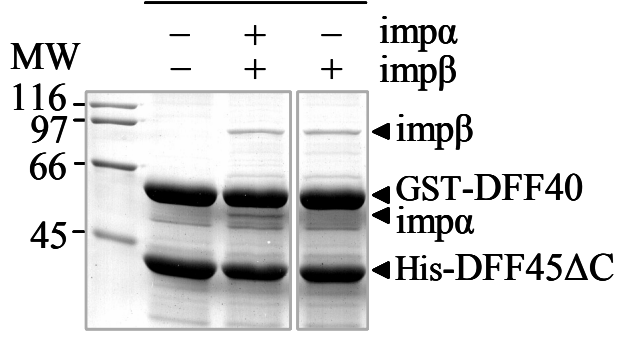

C

\section{binding of importins to \\ His-DFF40/ \\ GST-DFF45 $\Delta 306-331$}

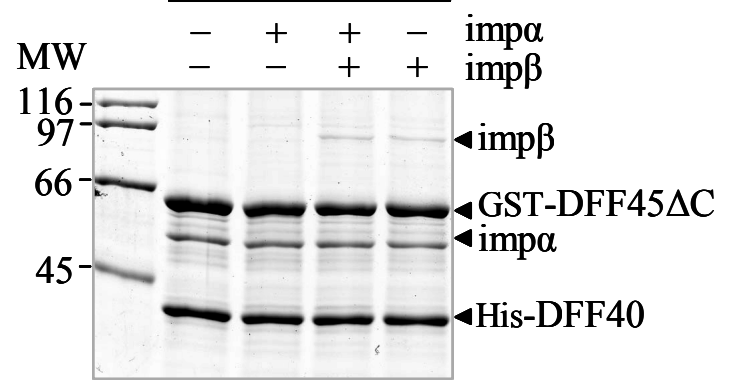

FIG. 14: The C-terminus of DFF45 is necessary for the interaction between the DFF complex and importin $\boldsymbol{\alpha} / \boldsymbol{\beta}$. $A$, Besides importin $\alpha / \beta$ none of the other import receptors was bound to GST-DFF45. Immobilized GST-DFF45 (45) was incubated with importin $\alpha$, importin $\alpha / \beta$, importin $\beta$, transportin (trn), importin 5 , importin 7 , importin 9 or importin 13, all from bacterial lysates. Bound fractions were analyzed by SDS-PAGE followed by Coomassie staining. $B$ and $C$, epitope-tagged C-terminally truncated DFF45 (DFF45 $\Delta 306-331$; DFF45 $\Delta$ C) was coexpressed with epitope-tagged DFF40 in $E$. coli. The purified GST-DFF40/His-DFF45 $\Delta 306-331$ complex $(B)$ or His-DFF40/GST-DFF45 $\triangle 306-331$ complex $(C)$ was immobilized on glutathione-Sepharose and incubated with importin $\alpha$, importin $\alpha / \beta$ and importin $\beta$ as indicated. Bound fractions were analyzed by SDS-PAGE followed by Coomassie staining. None of the used import receptors was bound efficiently to the truncated DFF40/DFF45 $\triangle 306-$ 331 complex. MW, molecular weight in kilodalton; imp (i), importin. 
Despite the weak binding of importin $\alpha / \beta$ to immobilized GST-DFF45, in vivo experiments had revealed an essential basic cluster for nuclear accumulation of the DFF complex (Fig. 11D and $E)$. Therefore, in vitro binding studies with DFF complexes containing C-terminally truncated DFF45 (DFF45 $\Delta 306-331$ ) were performed (Fig. 14). In contrast to wild type DFF, binding of imortin $\alpha / \beta$ to GST-DFF40/His-DFF45 $\Delta 306-331$ was abolished (Fig. 14B). The same observation was made with a complex in which the affinity tags were exchanged among the subunits (Fig. 14C). The results of these in vitro binding studies again demonstrate the necessity of the C-terminal tail of DFF45 for the nuclear targeting of the complex.

\subsection{The C-terminal tail of DFF40 interacts with importins and also functions as NLS}

Since a cluster of basic amino acids in DFF40 is essential for nuclear import of DFF in vivo and the binding of importin $\alpha / \beta$ to DFF45 is much weaker than to the DFF40/DFF45 complex, we further analyzed the function of DFF40 in nuclear transport of DFF. Firstly, GST-pull down assays with a DFF complex containing C-terminally truncated DFF40 were performed. For that purpose, GST-DFF40 $324-338$ was coexpressed with His-DFF45 in E. coli. The proper folding of the subunits and complex formation was analyzed in a plasmid cleavage assay (Fig. 15A) as described previously (for details see Materials and Methods). Upon activation of the purified GST-DFF40 $\Delta 324-338 /$ His-DFF45 complex by caspase-3, GST-DFF40 $\Delta 324-338$ was able to cleave plasmid DNA (Fig. 15A, lane 3-5). This demonstrates that the GST-DFF40 $\triangle 324-338 / H i s-$ DFF45 complex is functional. For in vitro binding studies GST-DFF40 $324-338 /$ His-DFF45 was immobilized on glutathione-Sepharose and incubated with importin $\alpha$ and importin $\beta$ from bacterial lysates. As shown in Fig. 15B, deletion of amino acids 324-338 of DFF40 abolished the binding of importin $\alpha / \beta$ to the DFF complex (compare Fig. $7 A$ ). Similar results were obtained for His-DFF40 $\Delta 324-338 /$ GST-DFF45 complex (Fig. $15 C$ and $D$ ) excluding the influence of affinitytags. Thus, these observations support the results of the in vivo transfection experiments (see Fig. $10 C$ and $D$ ) underlining the importance of the C-terminus of DFF40 for nuclear import. To examine whether the $\mathrm{C}$-terminal region of DFF40 is directly involved in importin $\alpha / \beta$-binding we next performed GST-pull down assays with DFF40 alone. To this end, immobilized GSTDFF40/His-DFF45 was treated with purified caspase-3 to cleave off DFF45. The activated GSTDFF40 was then incubated with import receptors. Among them, importin $\alpha / \beta$ was bound specifically to DFF40 (Fig. 16A) but importin $\alpha / \beta$-binding was less efficient than to the DFF complex (for comparison see Fig. 7A). In contrast to the DFF complex and monomeric DFF45, immobilized GST-DFF40 was additionally bound by importin $\beta$ with nearly the same efficiency 

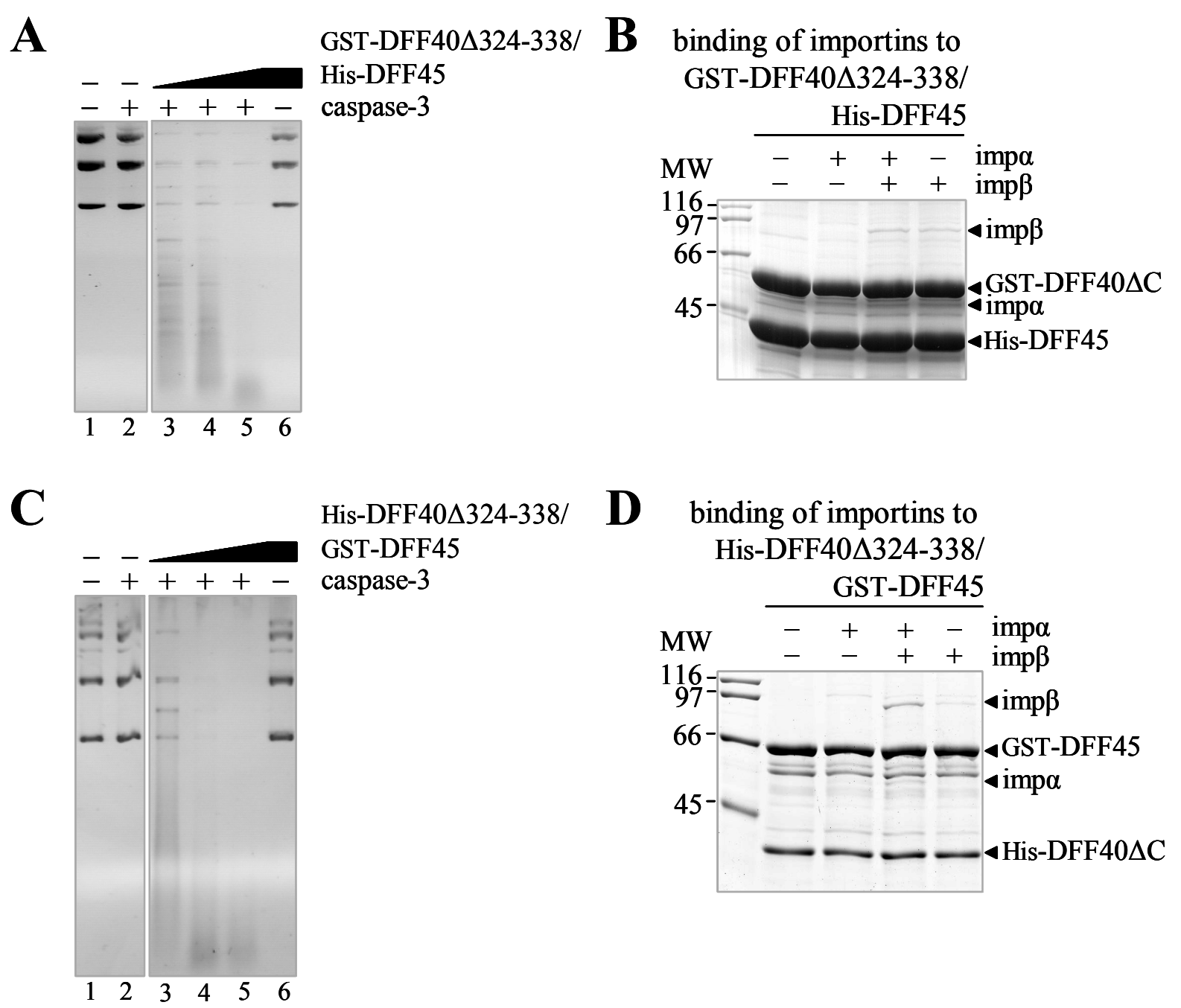

FIG. 15: The C-terminal tail of DFF40 contributes to the interaction between the DFF complex and importin $\boldsymbol{\alpha} / \boldsymbol{\beta}$. $A$ and $C$, the GST-DFF40 $\Delta 324-338 /$ His-DFF45 complex $(A)$ and the His-DFF40 $3324-338 / G S T-$ DFF45 complex $(C)$ can be activated by caspase-3 leading to the release of nucleolytically active GST-DFF40 $324-$ 338 or His-DFF40 $324-338$ respectively. Plasmid DNA was incubated with increasing amounts of GSTDFF40/His-DFF45 (lane 3-5 in panel A) or His-DFF40/GST-DFF45 (lane 3-5 in panel C) in the presence or absence of caspase- 3 for $2 \mathrm{~h}$ at $37^{\circ} \mathrm{C}$. Neither caspase-3 (lane 2 and 7) nor epitope-tagged DFF complex (lane 6) alone were able to cleave plasmid DNA. After phenol-chloroform extraction, the DNA was analyzed on a $1 \%$ agarose gel. The bands in lane 1 (plasmid control) represent the three topological forms of circular plasmid DNA. $B$ and $C$, epitopetagged C-terminally deleted DFF40 (DFF40 $324-338$ ) was coexpressed with epitope-tagged DFF45 in E. coli. The purified GST-DFF40 $\Delta 324-338 /$ His-DFF45 complex $(B)$ or His-DFF40 $\Delta 324-338 /$ GST-DFF45 complex $(D)$ was immobilized on glutathione-Sepharose and incubated with importin $\alpha$, importin $\alpha / \beta$ and importin $\beta$ as indicated. Bound fractions were analyzed by SDS-PAGE followed by Coomassie staining. None of the used import receptors was bound efficiently to the truncated DFF40 $324-338$ /DFF45 complex (for comparison with wild type DFF see also Fig $7 B$ and $D$ ). MW, molecular weight in kilodalton; aa, amino acids; imp (i), importin. 
as by importin $\alpha / \beta$ (Fig. 16A). Furthermore, the C-terminal region of DFF40 fused to GST [GSTDFF40(aa314-338)] interacts with importin $\alpha / \beta$ as well as with importin $\beta$ in a RanGTP-sensitive fashion (Fig. 16B). The binding of importin $\beta$ alone to the immobilized C-terminus of DFF40 was even stronger compared to importin $\alpha / \beta$-binding. These data suggest that the $\mathrm{C}$-terminal tail of DFF40 exhibits a NLS. However, this NLS alone can not account for the strong importin $\alpha / \beta$ binding to the DFF complex because this signal is hardly recognized within a DFF40/DFF45 complex containing C-terminally truncated DFF45 (see again Fig. 14B and C).
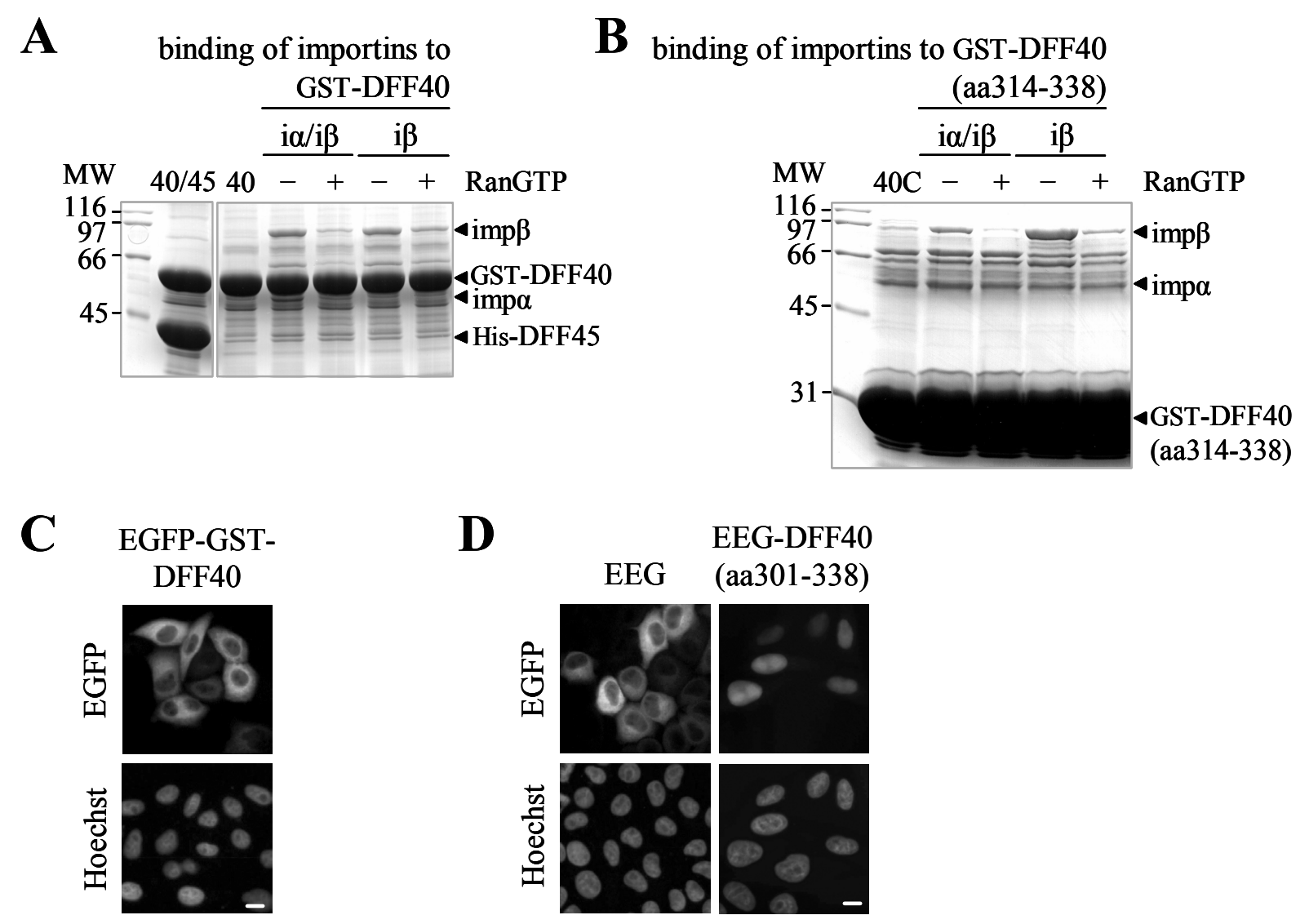

FIG. 16: The C-terminal tail of DFF40 contains a functional NLS. $A$ and $B$, amino acids 314-338 of DFF40 are responsible for the specific binding of importin $\alpha / \beta$ and importin $\beta$ to DFF40. Immobilized GSTDFF40/His-DFF45 complex (40/45) was incubated with caspase- 3 for 30 min at $30^{\circ} \mathrm{C}$ to cleave off DFF45 and thus activate DFF40. The immobilized activated GST-DFF40 (40) (A) and GST-fused amino acids 314-338 of DFF40 [GST-DFF40(aa314-338), 40C] (B) were incubated with importin $\alpha / \beta$ and importin $\beta$ in the absence or presence of $2 \mu \mathrm{M}$ RanGTP. Bound fractions were analyzed by SDS-PAGE followed by Coomassie staining. $C$ and $D, \mathrm{HeLa}$ P4 cells were transiently transfected with $(C)$ wild type EGFP-GST-DFF40 and $(D)$ amino acids 301-338 of DFF40 fused to EGFP-EGFP-GST (EEG). The subcellular distribution was examined $24 \mathrm{~h}$ after transfection by direct fluorescence and the DNA was counterstained with Hoechst. $C$, exclusively cytoplasmic localization of EGFP-GSTDFF40. D, the dominant cytoplasmic distribution of EEG (left panel) changed upon fusion to the C-terminus (aa301-338) of DFF40 (right panel), the localization now becoming largely nuclear. Scale bars represent $10 \mu \mathrm{m}$. MW, molecular weight in kilodalton; aa, amino acids; imp (i), importin. 
To characterize the function of the NLS of DFF40, in vivo transfection studies with EGFP-GSTtagged DFF40 were performed in HeLa P4 cells. Surprisingly, EGFP-GST-DFF40 was not transported into the nucleus as shown in Fig. 16C. However, the cytoplasmic EGFP-EGFP-GST fusion protein accumulated in the nucleus of transfected cells upon fusion to the C-terminal amino acids 301-338 of DFF40 [EGFP-EGFP-GST-DFF40(aa301-338); Fig. 16D]. This confirms that the C-terminus of DFF40 harbors a functional NLS. The cytoplasmic localization of monomeric EGFP-GST-DFF40 may be due to misfolding of DFF40 overexpressed in the absence of exogenous DFF45-chaperone.

In conclusion, in vivo transfection experiments with C-terminal regions of DFF40 and DFF45 indicate that both subunits exhibit functional NLSs. However, in pull down assays importin $\alpha / \beta$ was shown to bind the DFF40/DFF45 complex more strongly than the individual components. To explain the high affinity of importin $\alpha / \beta$ to the DFF complex compared to the single subunits, the interaction between the DFF complex and importin $\alpha / \beta$ was analyzed in more detail.

\subsection{Basic clusters in the C-terminal tails of DFF40 and DFF45 together presumably form a bipartite NLS}

Recently, Cingolani et al. (2002) identified a second cargo binding site (cargo binding site II) in importin $\beta$. This binding interface spans HEAT repeats 2-11 and is distinct from the IBB domain binding site (cargo binding site I). It was shown that the ncNLS of the parathyroid hormonerelated protein (PTHrP) interacts specifically with cargo binding site II and PTHrP ncNLS and IBB domain of importin $\alpha$ can bind simultaneously to importin $\beta$. The binding of activated DFF40 and GST-DFF40(aa314-338) not only to importin $\alpha / \beta$ but also to importin $\beta$ (Fig. $16 \mathrm{~A}$ and $B$ ) could point to the following transport model: Strong binding of importin $\alpha / \beta$ to the DFF40/DFF45 complex is accomplished by the cNLS of DFF45 via importin $\alpha$ using cargo binding site I of importin $\beta$ and simultaneous binding of the C-terminus of DFF40 to cargo binding site II of importin $\beta$. To examine this hypothesis, we performed GST-pull down assays with N-terminally deleted importin $\beta$ (Fig. 17). Immobilized GST-DFF40/His-DFF45 was incubated with importin $\alpha$ together with equal amounts of wild type importin $\beta$ and importin $\beta$ lacking either HEAT repeats 1-3 (importin $\beta \Delta 1-127$ ) or HEAT repeats 1-5 (importin $\beta \Delta 1-210$ ) (Fig. 17A). Deletion of these HEAT repeats would abolish a possible interaction between DFF40 and cargo binding site II. Therefore, binding of importin $\alpha / \beta$ to the DFF complex should be decreased in case of participation of this second cargo binding site in importin $\beta$. As shown in Fig. $17 B$, the $N$-terminal importin $\beta$ deletions slightly reduced binding of importin $\alpha / \beta$ to the immobilized GST-DFF40/His-DFF45 complex compared to wild type importin $\beta$. However, this 
effect was not significant, since binding of importin $\beta$ deletions to GST-nucleoplasmin was also reduced (Fig. 17C). GST-nucleoplasmin was used as control substrate for binding of importin $\alpha / \beta$ heterodimers containing the importin $\beta$ deletions. After all, this binding model can not explain the strong importin $\alpha / \beta$-binding to the DFF complex compared to the only weak and moderate interaction with monomeric DFF40 and DFF45.
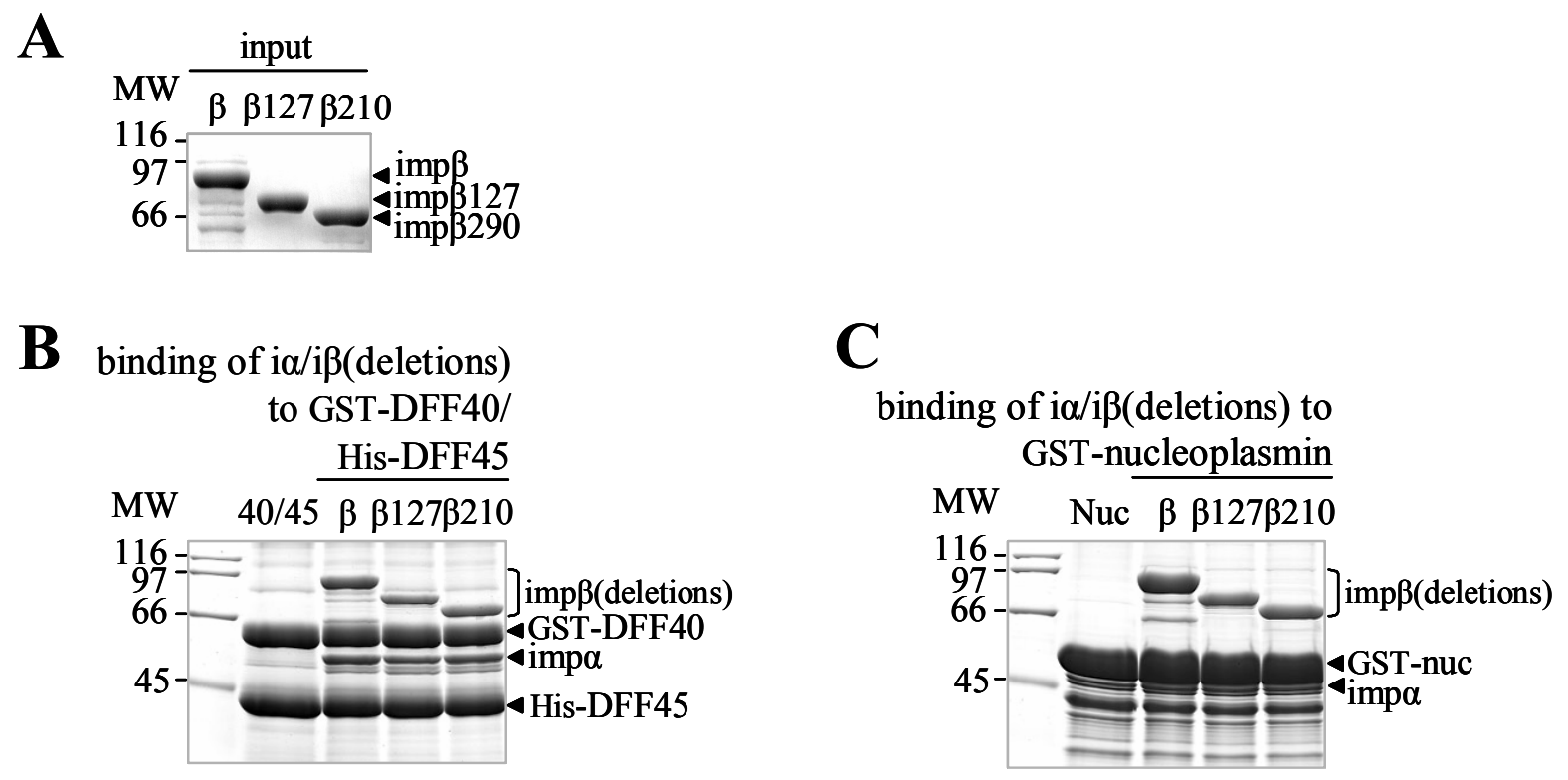

FIG. 17: The amino-terminal region of importin $\beta$ does not participate in the binding of importin $\boldsymbol{\alpha} / \boldsymbol{\beta}$ to the DFF complex. Immobilized GST-DFF40/His-DFF45 complex (40/45) (B) and GST-nucleoplasmin (nuc) $(C)$ were incubated with importin $\alpha$ from bacterial lysate together with purified recombinant wild type importin $\beta$, importin $\beta \Delta 1-127$ ( $\beta 127$ ) or importin $\beta \Delta 1-210$ ( $\beta 210$ ). Bound fractions were analyzed by SDS-PAGE followed by Coomassie staining. $10 \%$ of the wild type importin $\beta$ and importin $\beta$ deletions used for this interaction study are shown in panel $A$ (input). Amino-terminal deletions of importin $\beta$ did not significantly affect the binding of importin $\alpha / \beta$ to the immobilized GST-DFF40/His-DFF45 complex $(B)$ since reduced binding of the deletions can also be observed for the control GST-nucleoplasmin $(C)$. MW, molecular weight in kilodalton; imp (i), importin.

Since at least one basic cluster in each DFF subunit is required for nuclear import of the DFF complex another model for increased DFF-import receptor interaction could be following: Both essential basic clusters in DFF40 and DFF45 interact simultaneously with importin $\alpha$, mimicking a bipartite cNLS. To test this assumption, we generated an importin $\alpha$ mutant with a nonfunctional minor binding site. It was shown that substitution of glutamate at position 402 with arginine in Saccharomyces cerevisiae importin $\alpha$ (SRP1p) strongly decreases the binding to bipartite cNLSs. The interaction with monopartite cNLSs is thereby only slightly affected (Leung et al., 2003). Hence, we substituted arginine (R) for the corresponding glutamate (E) at 
position 388 of Xenopus laevis importin $\alpha 1$ ( $\alpha \mathrm{E} 388 \mathrm{R})$ and performed GST-pull down assays with this importin $\alpha$ mutant (Fig. 18). Nucleoplasmin was used as control substrate because it exhibits a bipartite cNLS. Binding of importin $\alpha / \beta$ containing importin $\alpha$ E388R to GST-nucleoplasmin was significantly reduced compared to wild type importin $\alpha$ (Fig. 18B). This nicely demonstrates that the importin $\alpha$ mutant has almost entirely lost its ability to bind bipartite cNLSs. A similar

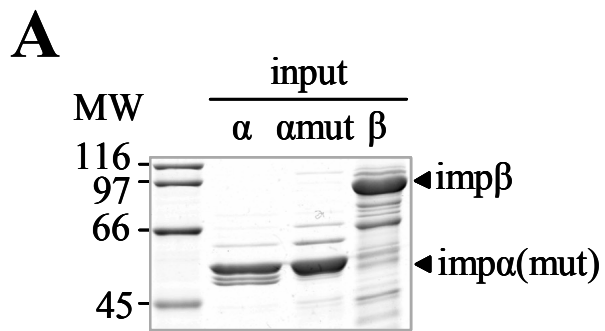

B

binding of $i \alpha(m u t) / i \beta$ to

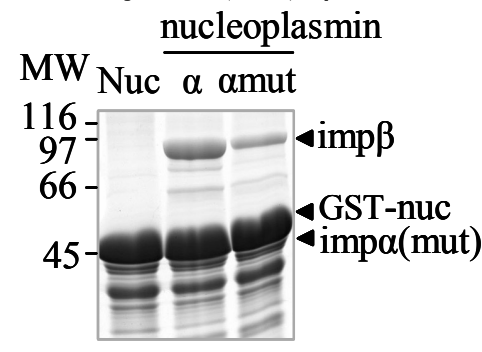

D

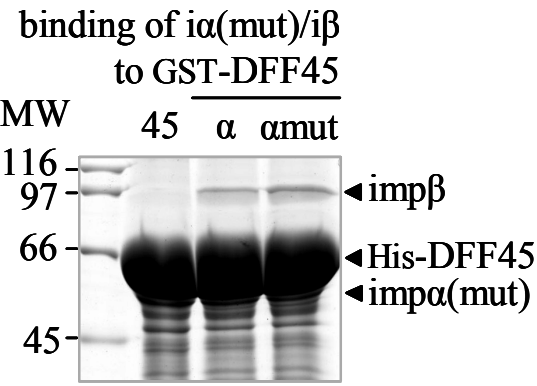

C binding of $i \alpha(m u t) / i \beta$

to GST-DFF40/

His-DFF45

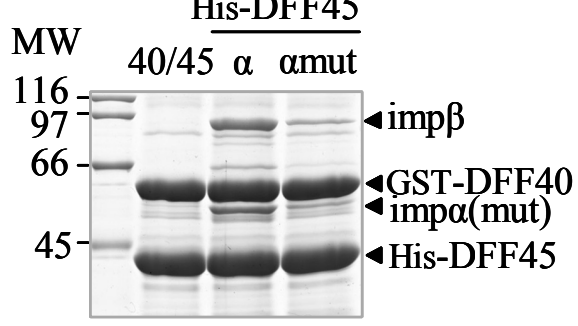

E binding of $i \alpha(m u t) / i \beta$ to

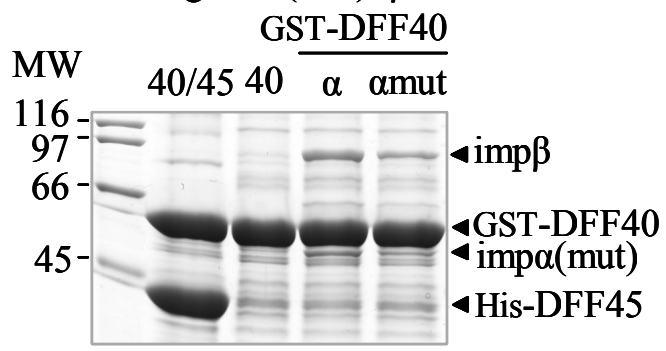

FIG. 18: The minor binding site of importin $\alpha$ is required for efficient binding of importin $\alpha / \beta$ to the DFF complex. GST-nucleoplasmin (nuc) (B), GST-DFF40/His-DFF45 complex (40/45) (C), and GST-DFF45 (45) $(D)$ were immobilized on glutathione-Sepharose. Immobilized GST-DFF40/His-DFF45 was incubated with caspase-3 for $30 \mathrm{~min}$ at $30^{\circ} \mathrm{C}$ to cleave off DFF45 and activate GST-DFF40 (40) (E). Immobilized proteins were incubated with purified recombinant importin $\alpha$ or importin $\alpha \mathrm{E} 388 \mathrm{R}$ ( $\alpha$ mut) together with importin $\beta .10 \%$ of the used transport receptors are shown in panel $A$ (input). Bound fractions were analyzed by SDS-PAGE followed by Coomassie staining. The binding of importin $\alpha \mathrm{E} 388 \mathrm{R} / \beta$ to nucleoplasmin $(B), \mathrm{DFF} 40 / \mathrm{DFF} 45(C)$, and activated DFF40 $(E)$ was reduced compared to importin $\alpha / \beta$. In contrast, importin $\alpha$ E388R did not affect the binding of importin $\alpha / \beta$ to DFF45 $(D)$. Note, when purified importin $\alpha$ was used instead of importin $\alpha$ from bacterial lysate weaker binding of importin $\alpha / \beta$ to immobilized DFF can be observed in general (see also Fig. $7 A$ ). MW, molecular weight in kilodalton; imp (i), importin. 
effect was observed for the interaction between importin $\alpha \mathrm{E} 388 \mathrm{R} / \beta$ and immobilized GSTDFF40/His-DFF45 (Fig. 18C), suggesting that the DFF complex exhibits indeed a bipartite cNLS. These data support the model according to which the basic clusters in DFF40 and DFF45 form a bipartite cNLS. In contrast, binding of importin $\alpha / \beta$ to GST-DFF45 alone was not affected using the importin $\alpha E 388 \mathrm{R}$ mutant (Fig. 18D). These results however tell two things; firstly the importin $\alpha \mathrm{E} 388 \mathrm{R}$ mutant is fully capable to bind monopartite cNLSs. Secondly, the C-terminal tail of DFF45 exhibits a cNLS which is monopartite.

Surprisingly, binding of importin $\alpha \mathrm{E} 388 \mathrm{R} / \beta$ to activated GST-DFF40 was also reduced (Fig. $18 E$ ) indicating that DFF40 itself possesses a bipartite cNLS. Previously, only one basic cluster (RxKRK) in the C-terminal region of DFF40 was identified to be essential for nuclear import of the DFF complex in transfected HeLa cells (see Fig. $11 B$ and $C$ ). In addition, the C-terminal tail of DFF40 does not contain an amino acid sequence homologous to the consensus motif for bipartite cNLS (2K/R-X $10-12-3 \mathrm{~K} / \mathrm{R}$; compare Fig. 11A). Therefore, the C-terminal sequence of DFF40 was searched for a second basic patch upstream of the essential amino acids. Two lysines at position 308 and 309 were found (Fig. 19A), however, the spacer between upstream residues and the downstream cluster comprises 17 amino acids. As mentioned earlier (chapter 1.2.3), in certain proteins such as hnRNP type 1 the linker region of a bipartite cNLS reaches a length of up to 32 amino acids (Romanelli and Morandi, 2002). Therefore, lysines at positions 308 and 309 in DFF40 may as well form a bipartite cNLS with the downstream cluster RxKRK. This putative bipartite cNLS might be responsible for decreased binding of importin $\alpha E 388 \mathrm{R} / \beta$ to activated DFF40 and the DFF complex (Fig. 18C and E). To test this possibilty, the potential role of lysine 308 and 309 in the nuclear transport of the DFF complex was analyzed next.

We generated a DFF40 mutant (DFF40mutC) substituting the lysines at position 308 and 309 for alanine (Fig. 19A). The subcellular localization of a DFF complex containing DFF40mutC was examined in HeLa P4 cells. For that, EGFP-GST-tagged DFF40mutC was coexpressed with RFP-tagged DFF45 and vice versa. The results of these in vivo transfection studies are summarized in Fig. 19. The dominant nuclear localization of the DFF40/DFF45 complex was not affected by substitution of lysine residues 308 and 309 neither using EGFP-GST-tagged DFF40 (Fig. 19B) nor RFP-tagged DFF40 (Fig. 19C). This demonstrates that these two basic residues are not required for nuclear accumulation of DFF. To confirm these data and to further characterize the cNLS in DFF40, pull down assays with this DFF40 mutant were additionally performed. For that, GST-DFF40 or GST-DFF40mutC were coexpressed with His-DFF45 in E. coli. Immobilized GST-DFF40/His-DFF45 and GST-DFF40mutC/His-DFF45 complexes were 
A

DFF40wt 301 LVHIVCHKKTTHKLNCDPSRIYKPQTRLKRKQPVRKRQ 338

DFF40mutC 301 LVHIVCHAATTHKLNCDPSRIYKPQTRLKRKQPVRKRQ 338

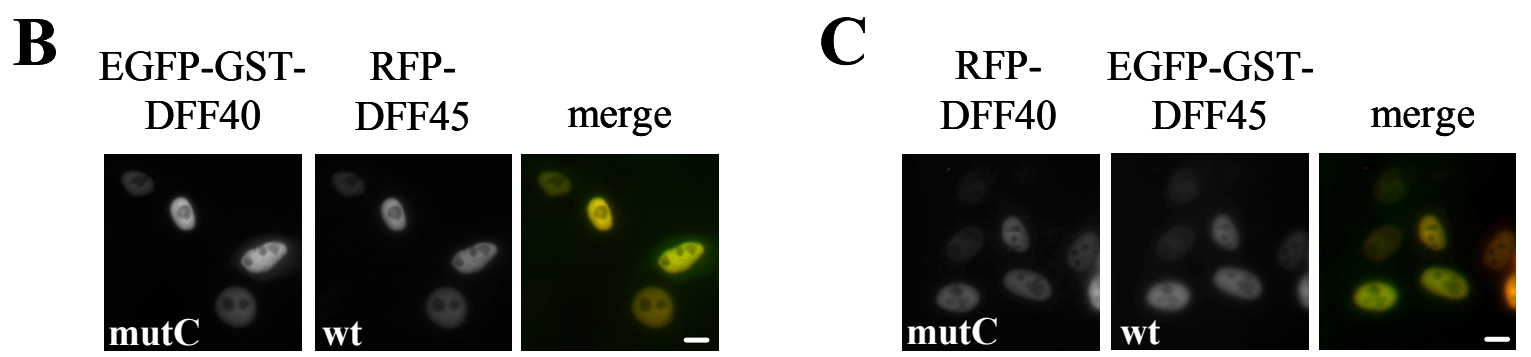

FIG. 19: Nuclear accumulation of the DFF complex does not require lysine 308 and 309 of DFF40.

Mutated DFF40 with alanine substitutions of two basic amino acids upstream of the essential basic cluster (DFF40mutC) was fused to EGFP-GST or RFP and was cotransfected with DFF45 into HeLa P4 cells. The subcellular localization of the subunits was examined $24 \mathrm{~h}$ after transfection by direct fluorescence. The overlap between the green EGFP-fusion protein and the red RFP-fusion protein is shown in yellow (merge). Scale bars represent $10 \mu \mathrm{m}$. $A$, amino acids 301-338 of DFF40. Basic amino acids of the putative unusual bipartite cNLS are indicated by bold characters and mutated amino acids by red characters. $B$ and $C$, DFF40mutC fused to EGFP-GST $(B)$ or to RFP $(C)$ was cotransfected with wild type RFP-DFF45 (B) or EGFP-GST-DFF45 (C). Mutation of the two positively charged amino acids at position 308 and 309 in DFF40mutC did not affect the nuclear localization of the DFF complex.

then used as bait for importin $\alpha$ and importin $\beta$, both from bacterial lysates (Fig. 20A). Mutation of lysine 308 and 309 in DFF40 (DFF40mutC) did not affect binding of the importin $\alpha / \beta$ heterodimer to the DFF complex. The interaction of importin $\alpha / \beta$ with DFF40mutC/DFF45 was similar to that with wild type DFF40/DFF45 and supports the results of the in vivo transfection studies mentioned above. A functional relevance of these two lysine residues in DFF40 regarding the nuclear translocation of the DFF complex can therefore be excluded. Furthermore, the binding of importin $\alpha / \beta$ to activated DFF40mutC was also not influenced by substitution of lysine residues 308 and 309 (Fig. 20B). This result argues against a participation of these amino acids in a bipartite cNLS. Whether DFF40 does indeed contain a bipartite cNLS as suggested by the binding studies with importin $\alpha E 388 \mathrm{R}$ (see again Fig. 18E) or not as indicated by the use of DFF40mutC (see Fig. 20B) remains currently unanswered. On one hand other basic amino acids upstream of the essential basic cluster in DFF40 could be involved in importin $\alpha / \beta$-binding. On the other hand the importin $\alpha \mathrm{E} 388 \mathrm{R}$ mutant could have a general binding deficiency towards cNLSs (including monopartite signals) leading to a misinterpretation regarding the signal type. Further experiments will be necessary to answer this question. 

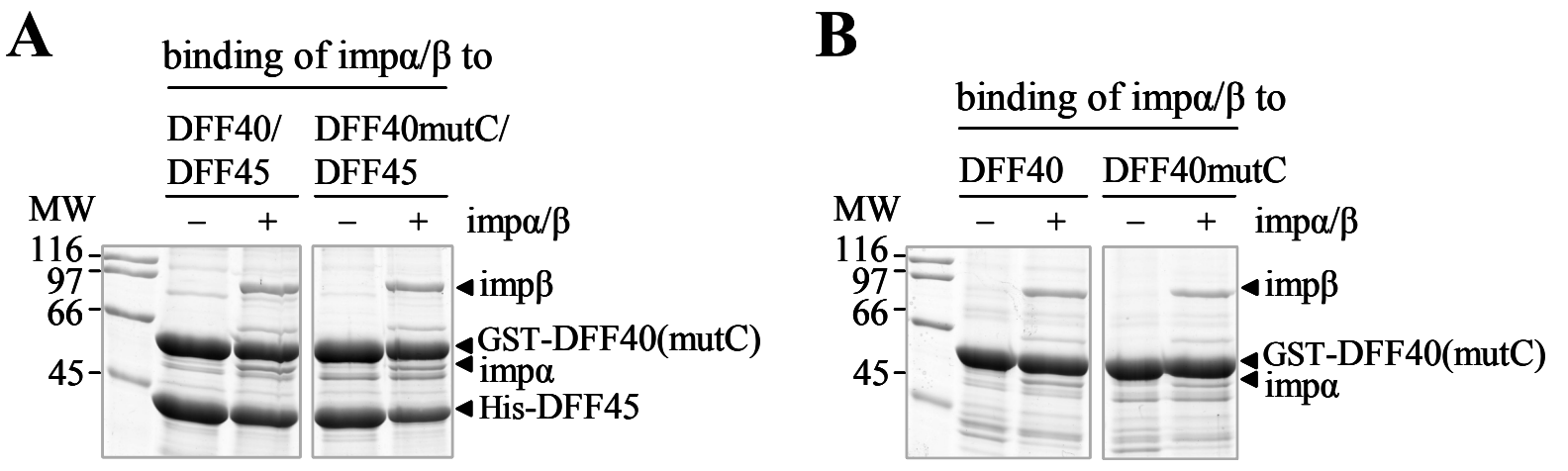

FIG. 20: Lysines 308 and 309 in DFF40 are not required for importin $\alpha / \beta$ binding. Mutation of the two positively charged amino acids at position 308 and 309 in DFF40mutC did not influence binding of the importin $\alpha / \beta$ heterodimer to the DFF complex $(A)$ or to activated DFF40 $(B)$. $A$, immobilized GST-DFF40/His-DFF45 complex and GST-DFF40mutC/His-DFF45 complex were incubated with importin $\alpha$ and importin $\beta$ from bacterial lysates. Bound fractions were analyzed by SDS-PAGE followed by Coomassie staining. $B$, binding of importin $\alpha / \beta$ to activated GST-DFF40 and activated GST-DFF40mutC. Immobilized GST-DFF40/His-DFF45 complex and GSTDFF40mutC/His-DFF45 complex were incubated with caspase-3 for 30 min at $30^{\circ} \mathrm{C}$ to cleave off DFF45. After washing, the immobilized GST-DFF40 and GST-DFF40mutC were incubated with importin $\alpha / \beta$ and importin $\beta$ from bacterial lysates. Bound fractions were analyzed by SDS-PAGE followed by Coomassie staining. MW, molecular weight in kilodalton; imp (i), importin.

The data obtained in this study can be summarized as follows: In vitro pull down assays and in vitro import assays revealed that nuclear transport of the DFF complex is mediated by the importin $\alpha / \beta$-pathway. At least one basic cluster required for the nuclear translocation of the DFF complex was identified in the C-terminal region of each subunit, DFF40 and DFF45. Furthermore, the sequence element in DFF45 that is essential for the nuclear accumulation of the DFF complex additionally functions as monopartite cNLS for the nuclear import of monomeric DFF45 mediated by importin $\alpha / \beta$. In vitro binding studies and in vivo transfection experiments indicate that DFF40 contains a functional NLS as well. However, differences in the binding of importin $\alpha / \beta$ to the DFF complex (strong), active DFF40 (moderate) and monomeric DFF45 (weak) suggest that the basic cluster in DFF40 and DFF45 together form an intermolecular cNLS that mediates the interaction with importin $\alpha / \beta$. 


\section{Discussion}

One of the biochemical hallmarks of apoptosis is internucleosomal DNA fragmentation mediated by the endonuclease DFF40. In healthy cells nuclease activity of DFF40 is inhibited through interaction with its specific chaperone DFF45 and thereby the DFF complex is formed. This complex probably consists of two DFF40/DFF45 heterodimers (Lechardeur et al., 2005). In search for the biological function of apoptotic DNA fragmentation, an important role was recently described. Yan et al. (2006) have demonstrated that DFF40-mediated DNA fragmentation is involved in maintaining genomic stability and preventing tumorigenesis (Yan et al., 2006a). This report shows that inhibition or loss of DFF40 significantly increases spontaneous as well as induced gene mutations, gene amplifications and chromosomal instability. Cells deficient in DFF40 showed an increased survival after irradiation. Therefore, DFF40 contributes to the destruction of cells with damaged DNA which preserves genomic stability (Yan et al., 2006a).

Apoptotic DNA fragmentation occurs in the nucleus (early stage) or nuclear remnants (late stage), respectively. It was reported that DFF40 translocates into the nucleus in complex with DFF45 (Lechardeur et al., 2000; Samejima and Earnshaw, 2000). While the C-termini of both subunits were shown to be involved in nuclear translocation of the DFF complex (Lechardeur et al., 2000; Samejima and Earnshaw, 2000) NLSs and the nuclear import mechanism were not characterized. Therefore, the aim of this study was to analyze the nuclear transport pathway of the DFF complex in detail. In particular, the dependence on nuclear transport receptors was investigated and sequence elements that mediate nuclear import were characterized.

\subsection{The DFF complex enters the nucleus via the classical importin $\alpha / \beta$-pathway}

Although the majority of nuclear proteins crosses the nuclear membrane in association with soluble transport receptors some proteins overcome the permeability barrier of the NPC by themselves. For instance, extracellular signal-regulated kinase (ERK) 2 enters the nucleus by a transport-receptor independent mechanism through direct interaction with nuclear pore proteins (Whitehurst et al., 2002). To characterize the transport pathway of the DFF complex, a recombinant purified DFF complex containing an EGFP-tagged subunit was used in the in vitro nuclear import assay. In this assay, established by Adam et al. (1990), permeabilization of cell membranes using digitonin allows manipulation of nuclear import processes by substitution of endogenous cytosolic components. Despite carrying a fluorescent tag the DFF complex could be activated by caspase- 3 which proved its functionality. The use of this functional EGFP-tagged DFF complex in the in vitro nuclear import assay (applying different conditions) clearly 
demonstrated that DFF traverses the nuclear envelope in a receptor-mediated (reticulocyte lysate), energy-dependent (apyrase, $4^{\circ} \mathrm{C}$ ) fashion that requires access to nuclear pore proteins (WGA) (Fig. 6B). Additional in vitro experiments followed to address the question which nuclear transport receptors mediate the observed active import of the DFF complex into nuclei of permeabilized cells. Eventually, in vitro binding studies combined with in vitro import assays revealed that the importin $\alpha / \beta$ heterodimer is responsible for nuclear import of the DFF complex. Firstly, out of the tested import receptors only importin $\alpha / \beta$ was efficiently bound to immobilized DFF40/DFF45 complexes in GST-pull down assays (Fig. 7). Secondly, this importin $\alpha / \beta-D F F$ interaction led to nuclear accumulation of a fluorescently tagged DFF complex in digitoninpermeabilized HeLa cells (Fig. 9). However, nuclear uptake of the DFF complex reconstituted with recombinant importin $\alpha / \beta$ was less efficient than with reticulocyte lysate, used as source of native import receptors. On one hand, this might be due to the interaction of DFF with additional import receptors not analyzed in GST-pull down assays. On the other hand, we demonstrated that the DFF complex specifically interacts with different Xenopus leavis (X.) importin $\alpha$ isoforms, namely importin $\alpha 1, \alpha 2$ and $\alpha 5.1$ (Fig. 8). Among these importin $\alpha$ subtypes $X$. importin $\alpha 1$ and $\alpha 2$ belong to the Rch1 subclass (Fig. 8A) whose members are responsible for a large number of importin $\alpha / \beta$-mediated translocations (for examples, see Jans et al., 2000). The better nuclear uptake of DFF in the presence of reticulocyte lysate may therefore be explained by the broader range of suitable importin $\alpha$ molecules. In contrast, either $X$. importin $\alpha 1$ or human importin $\alpha 2$, which represents the Rch1 class in humans, was used exclusively for the in vitro nuclear import assay with purified recombinant import receptors. Regarding the binding of $X$. importin $\alpha$ isoforms it is remarkable that $X$. importin $\alpha 3$ did not specifically bind to the DFF complex although it shows the highest sequence homology to human importin $\alpha 2$ (compare Fig. $8 A$ ). However, $X$. importin $\alpha 3, \alpha 4$ and $\alpha 5.2$ were N-terminally myc-tagged and none of these isoforms interacted specifically with DFF. In contrast, the three tag-free $X$. importin $\alpha$ subtypes bound to the DFF complex in a RanGTP-sensitive manner. Therefore, it can not be excluded that the myc-tag interferes with binding of importin $\alpha$ to the DFF complex. For instance, the interaction between importin $\beta$ and the IBB domain of importin $\alpha$, also N-terminally located, could be inhibited. This might explain why $X$. importin $\alpha 3$ and $\alpha 5.2$ did not interact with the DFF complex in comparison to their closest homologues $X$. importin $\alpha 1, \alpha 2$ and $\alpha 5.1$, respectively. Although some cargoes can be transported into the nucleus by different importin $\alpha$ isoforms, a number of reports demonstrate that nuclear accumulation of several substrates is specifically mediated by a particular importin $\alpha$ subtype (Friedrich et al., 2006; Jans et al., 2000, and see references therein). For instance, while nucleoplasmin is imported into the nucleus by all human 
importin $\alpha$ isoforms, importin $\alpha 3$ specifically mediates the nuclear translocation of RCC1 (Kohler et al., 1999; Quensel et al., 2004). Importin $\alpha 3$ and $\alpha 4$ (Qip-1 class) are responsible for the nuclear accumulation of NF- $\mathrm{BB}$ transcription factors (Fagerlund et al., 2005) whereas STAT (signal transducer and activator of transcription) 1 and STAT 2 are specifically transported into the nucleus by importin $\alpha 1$ (NPI-1) (Sekimoto et al., 1997). Furthermore, Yasuhara et al. (2007) recently demonstrated that nuclear import of transcription factors involved in neuronal differentiation depends on specific importin $\alpha$ subtypes. The expression of these importin $\alpha$ isoforms is strictly regulated during differentiation and switching of importin $\alpha$ subtype expression is coordinated with regulated expression of these transcription factors (Yasuhara et al., 2007). Therefore, certain importin $\alpha$ isoforms are directly involved in coordination of neural differentiation.

Among the $X$. importin $\alpha$ isoforms $X$. importin $\alpha 1$ and $\alpha 2$ showed the highest binding competence for the DFF complex (Fig. 8). These two $X$. importins belong to the Rch1 subclass. In humans the Rch1 subclass is represented by importin $\alpha 2$ which therefore might be the primary importin $\alpha$ isoform for nuclear accumulation of the endogenous DFF40/DFF45 complex. Nuclear uptake of DFF in the presence of recombinant human importin $\alpha 2$ in combination with importin $\beta$ (Fig. 9) strongly supports this assumption. Nevertheless, in vitro binding studies have to be performed with untagged $X$. importin $\alpha 3, \alpha 4$ and $\alpha 5.2$ in addition, to elucidate whether specific binding of these importin $\alpha$ isoforms to the DFF complex was prevented by the N-terminal myctag or not.

In general, proteins interacting with importin $\alpha$ exhibit classical NLSs (cNLSs) resembling either the monopartite type of SV40 large T antigen (Kalderon et al., 1984a) or the bipartite type of nucleoplasmin (Dingwall et al., 1988; Robbins et al., 1991). Hence, the identification of importin $\alpha / \beta$ as functional transport receptor for the DFF complex indicates that the DFF complex contains at least one cNLS. Classical NLSs were initially described as short sequences enriched in positively charged amino acids (Dingwall and Laskey, 1991; Robbins et al., 1991). Based on several structural studies the originally defined consensus sequences of monopartite and bipartite cNLSs were modified. Analysis of the interaction between importin $\alpha$ and the SV40 large $\mathrm{T}$ antigen NLS (KKKRKV; referred to as P1-P6) revealed that lysine P2 is strictly required for binding of importin $\alpha$ (Conti et al., 1998; Fontes et al., 2000; Hodel et al., 2001). The amino acid positions P3 and P5 are also important for this interaction. Thus, the current view of a monopartite cNLS is following: Firstly, the signal exhibits the central consensus motif K-K/R-XK/R (P2-P5) (Chelsky et al., 1989). Secondly, amino acid residues that are compatible with sharp turns like proline or glycine upstream of the consensus motif and a small hydrophobic amino 
acid followed by an acidic residue downstream of the consensus are preferred (Conti, 2002). In contrast to the monopartite cNLS, the sequence requirements for bipartite cNLS are less stringent because of multiple interactions between NLS and importin $\alpha$ surface groove (Conti and Kuriyan, 2000; Dingwall and Laskey, 1991). Bipartite cNLSs like the nucleoplasmin NLS consist of two basic clusters connected by a 10-12 amino acid spacer following the loose consensus 2K/R-X $10-12-3 \mathrm{~K} / \mathrm{R}$ (Conti et al., 1998; Dingwall and Laskey, 1991; Robbins et al., 1991). In certain cases the amino acid spacer between the two basic stretches might reach a length of up to 32 amino acid residues (Luo and Shibuya, 2001; Romanelli and Morandi, 2002). High affinity binding of importin $\alpha / \beta$ to bipartite cNLSs is predicted when a more strict consensus sequence KR-X $10-12-\mathrm{KRRK}$ is present (Fontes et al., 2003). However, this type of sequence might not be optimal since a functional cNLS not only has to bind importin $\alpha$ but also must be efficiently released from it in the nucleus. Furthermore, even if the downstream cluster of a bipartite cNLS resembles a monopartite cNLS, this amino acid stretch is not able to mediate translocation of a protein into the nucleus (Dingwall et al., 1988). Hence, bipartite cNLSs are characterized by simultaneous interactions of the two basic clusters with importin $\alpha$.

Interestingly, besides the NLS also the protein context of a cargo contributes to the recognition of specific importin $\alpha$ isoforms (Friedrich et al., 2006). In general, monopartite cNLSs and the larger downstream basic cluster of bipartite cNLSs bind to the major binding site of importin $\alpha$ comprising ARM repeats 2-4 (reviewed in Conti, 2002, see also Fig. 3). The smaller upstream basic cluster of bipartite cNLSs interacts with the minor binding site of importin $\alpha$ located in ARM repeats 7 and 8 . However, there are also exceptions regarding the binding of cargoes to importin $\alpha$. Some substrates recognize other regions of importin $\alpha$ via NLSs different from classical types. For instance, transcription factors STAT 1/2 and influenza A virus nucleoprotein bind to ARM repeats 8-9 and 7-9 of human importin $\alpha 1$ (Melen et al., 2003). Phospholipid scramblase 1 (PLSCR1) interacts with importin $\alpha$ ARM repeats 1-4 via a NLS enriched in hydrophobic amino acid residues (Chen et al., 2005). In this case, major binding site of importin $\alpha$ and PLSCR1 binding site of importin $\alpha$ partially overlap.

For the characterization of sequence elements required for nuclear targeting of the DFF complex fluorescently tagged wild type, C-terminally truncated, and mutant DFF subunits were coexpressed in HeLa cells. The coexpression of C-terminal deletions of both DFF subunits with the corresponding wild type subunit clearly showed the importance of both C-termini for nuclear accumulation of DFF (Fig. 10). These results strongly support previous data by Lechardeur et al. (2000) who had already claimed that the C-termini of the two subunits are required for nuclear import of the DFF complex. The next step was then to identify amino acid residues responsible 
for nuclear accumulation of the DFF complex. For that purpose, basic amino acids in the C-terminal tails of DFF40 and DFF45 were substituted by alanine. In vivo transfection studies with these mutants revealed that at least one basic cluster in the C-terminal region of each subunit, DFF40 (RxKRK) and DFF45 (KRxR), is essential for nuclear localization of the DFF complex (Fig. 11). Mutation of two lysines at positions 307 and 313 of DFF45 (DFF45mutA) lead to contradictory results regarding the involvement of these residues in nuclear targeting of the DFF complex (Fig. 11). In more detail, EGFP-GST-DFF45mutA coexpressed with RFPDFF40 showed no effect on nuclear localization of the DFF complex (Fig. 11D) while RFPDFF45mutA in complex with EGFP-GST-DFF40 remained rather cytoplasmic in transfected cells (Fig. 11E). Another co-transfection experiment in which RFP-DFF45mutA was overexpressed with EGFP-GST-DFF40mutB, however, indicated again that these two lysine residues in DFF45 are not necessary for translocation of the DFF complex. A dominant nuclear localization pattern of the overexpressed DFF complex was clearly visible in this experiment (Fig. 11F). Taken together, these three in vivo transfection experiments point to no significant role of the lysine residues at position 307 and 313 in DFF45 for nuclear entry of the DFF complex. However, additional experiments are indispensable to finally resolve the somewhat contradictory results. For instance, the requirement of K307 and K313 in DFF45 could be analyzed in GST-pull down assays with a mutated DFF complex in which these positively charged amino acids are substituted in DFF45.

To test whether the fluorescent tags (EGFP-GST, RFP) fused to the DFF subunits influenced their capability to interact with each other, we performed GST-pull down assays. After coexpression of fluorescently labeled DFF subunits in HeLa cells, RFP-tagged subunits always coprecipitated with EGFP-GST-tagged subunits (Fig. 9 and 12). Additionally, interaction of endogenous DFF45 with EGFP-GST-DFF40 was also observed. In contrast, endogenous DFF45 was not retained when EGFP-GST-DFF45 was coexpressed with RFP-DFF40 subunits. This observation points to a dimeric structure of the DFF complex under these conditions. Regarding its composition, the DFF complex is however believed to consist of two DFF40/DFF45 heterodimers (Lechardeur et al., 2005). This tetrameric structure was postulated based on results of (i) size exclusion chromatography of endogenous and eukaryotically overexpressed DFF complexes and (ii) in vivo transfection experiments with HA-, FLAG- and myc-tagged subunits (Lechardeur et al., 2005). If the DFF complex indeed consists of two heterodimers, endogenous DFF45 should also be part of overexpressed EGFP-GST-DFF45/RFP-DFF40 complexes. Since this could not be observed our results do not support the tetrameric structure model. In addition, Woo and colleagues estimated the molecular weight of the DFF40/DFF45 complex by gel 
filtration (Woo et al., 2004). Their recombinant, purified complex had also only the molecular weight of a heterodimer and revealed a 1:1 stoichiometry between DFF40 and DFF45. Alternatively, in our experiments the presence of EGFP-GST-fused subunits could lead to homodimerization mediated by GST which would not allow the integration of endogenous DFF45 into a DFF tetramer. Assembly of the tetrameric DFF complex is thought to be mediated at least in part by the N-terminally located CIDE domain of DFF40 (Lechardeur et al., 2005). Since the relatively large fluorescent tags are N-terminally attached they might sterically hinder the interaction between DFF40/DFF45 dimers through their CIDE domains. Accordingly, the fluorescence tags would not affect heterodimerization between the DFF subunits but prevent tetramerization. Formation of dimeric complexes containing endogenous DFF45 implies that both mutated and wild type DFF complexes are present in cells cotransfected with mutant DFF45 and wild type DFF40. Since the wild type DFF complex is mainly located in the nucleus of healthy cells one would expect that a complex consisting of exogenous EGFP-GST-DFF40 and endogenous DFF45 also accumulates in the nucleus. Surprisingly, green fluorescence of EGFP-GST-DFF40 was exclusively observed in the cytoplasm of transfected cells (Fig. 10C lower panel; Fig. 11E). Obviously, endogenous DFF45 can not compensate for the lack of overexpressed, exogenous DFF45.

In summary, on one hand in vivo transfection studies clearly showed that at least one positively charged cluster in the C-terminal tail of each DFF subunit is necessary for nuclear uptake of the DFF complex. In vitro nuclear import assays with recombinant transport receptors on the other hand revealed that importin $\alpha / \beta$ mediates the nuclear import of the DFF complex. To correlate in vivo and in vitro data importin binding studies with C-terminally truncated DFF complexes were performed. These GST-pull down experiments uncovered that the C-terminal regions of both subunits, DFF40 and DFF45, are necessary for binding of importin $\alpha / \beta$ (Fig. 14 and 15). The loss of one C-terminal tail in either DFF40 or DFF45 almost completely abolished binding of importin $\alpha / \beta$. In conclusion, the basic cluster in the $\mathrm{C}$-terminus of each subunit, DFF40 (RxKRK) and DFF45 (KRxR), is essential for importin $\alpha / \beta$ recognition and subsequent nuclear transport of the DFF40/DFF45 complex. To further characterize these two basic sequence elements involved in nuclear entry of the DFF complex, we also analyzed the individual DFF subunits with regard to their import receptor binding in vitro and nuclear transport in vivo. The presence of essential basic amino acid stretches in each DFF subunit raised the question whether these clusters within the C-terminal regions of DFF40 and DFF45 represent independent classical NLSs. In order to examine the cNLS properties of the basic stretches in the two DFF 
subunits in vivo transfection experiments and in vitro binding assays were performed with monomeric DFF40 and DFF45.

\subsection{Nuclear transport of monomeric DFF45 is mediated by importin $\alpha / \beta$}

Firstly, the subcellular localization of monomeric DFF45 was analyzed using in vivo transfection studies with fluorescently labeled DFF45. Besides wild type EGFP-GST-DFF45, C-terminally truncated and mutated EGFP-GST-DFF45 were overexpressed in HeLa cells. These localization experiments demonstrated that the C-terminal tail, in particular the basic cluster $\mathrm{KRxR}$, is essential for nuclear accumulation of monomeric DFF45 (Fig. 13A). Furthermore, fusion of the C-terminus of DFF45 (aa 306-331) to the cytoplasmic protein EGFP-EGFP-GST changed its subcellular localization, becoming nuclear (Fig. 13B). Hence, the C-terminal region of DFF45 is not only essential but also sufficient for nuclear uptake of monomeric DFF45. Additional in vitro binding studies with immobilized GST-DFF45 as well as the C-terminal tail of DFF45 alone fused to GST revealed that importin $\alpha / \beta$ was the only import receptor that specifically bound (Fig. $13 C$ and $D$ and Fig. 14). Therefore, it is reasonable to assume that (i) importin $\alpha / \beta$ mediates nuclear accumulation of monomeric DFF45 and (ii) the C-terminal tail of DFF45 exhibits a cNLS that confers nuclear uptake of DFF45. Furthermore, the basic cluster KRxR is not only required for nuclear import of the DFF complex but is also the key element of the NLS in DFF45. This amino acid stretch fulfills the consensus motif for a monopartite cNLS but additional sequence requirements are not fully satisfied. For instance, position P6 is occupied by a polar glutamine instead of a small hydrophobic amino acid (see Fig. 11A). In addition, the importin $\alpha$ binding pocket for P1 prefers lysine or arginine, however, proline as in the case of the DFF45 sequence is also tolerated (Fontes et al., 2003). These differences from preferred amino acids for monopartite cNLSs might explain why monomeric DFF45 only weakly interacts with importin $\alpha / \beta$. In contrast, sequence requirements for bipartite cNLS are less stringent because of multiple interactions between cNLS and importin $\alpha$. Therefore, the C-terminal tail of DFF45 might exhibit a bipartite cNLS as it was previously proposed (Lechardeur et al., 2000). To test this assumption, we substituted the additional basic amino acids, R307 and K313, in the C-terminal region (aa 306-331) of DFF45 with alanine. The nuclear localization of monomeric DFF45 containing these mutations was only slightly decreased in transfected HeLa cells (Fig. $13 A$ ). Since both basic clusters of bipartite cNLSs are necessary for importin $\alpha$-binding and nuclear import (Dingwall et al., 1988) these results argue against a bipartite cNLS in the C-terminus of DFF45. Furthermore, the homology of this region with the consensus motif for bipartite cNLS is rather small because R307 and K313 are not adjacent but separated by six 
amino acid residues. Therefore, only one of these amino acids could theoretically bind to the minor binding site of importin $\alpha$. In contrast, Robbins et al. (1991) demonstrated that two basic amino acids in the upstream cluster of bipartite cNLS are required for efficient nuclear transport. Additionally, all available structures of complexes composed of bipartite cNLS peptides and importin $\alpha$ show the binding of two basic residues at the minor binding site (Fontes et al., 2003). Thus, nuclear import of monomeric DFF45 is most likely mediated by a monopartite cNLS. This assumption is strongly supported by additional GST-pull down assays with an importin $\alpha$ minor binding site mutant (importin $\alpha \mathrm{E} 388 \mathrm{R}$ ). The interaction between importin $\alpha \mathrm{E} 388 \mathrm{R} / \beta$ and monomeric DFF45 was not affected, as expected for a monopartite cNLS (Fig. 18).

DFF45 is well conserved among species showing 34\% sequence identity between human, mouse and bovine DFF45 (Fig. 21). The C-terminal regions of mouse and bovine DFF45 harbor a stretch of basic amino acids, very similar to the essential basic cluster in human DFF45, that fulfills the consensus sequence for a monopartite cNLS. Therefore, it is reasonable to assume that the nuclear entry of mouse and bovine DFF45 is also mediated by this monopartite cNLS. Overall, the nuclear import pathway of DFF45 might be conserved among species. However, in contrast to the C-terminal tail of human and bovine DFF45 the C-terminus of mouse DFF45 additionally fulfills the requirements for a bipartite cNLS since two adjacent arginines are located ten amino acid residues upstream of the basic sequence KRAK (Fig. 21). Thus it is also

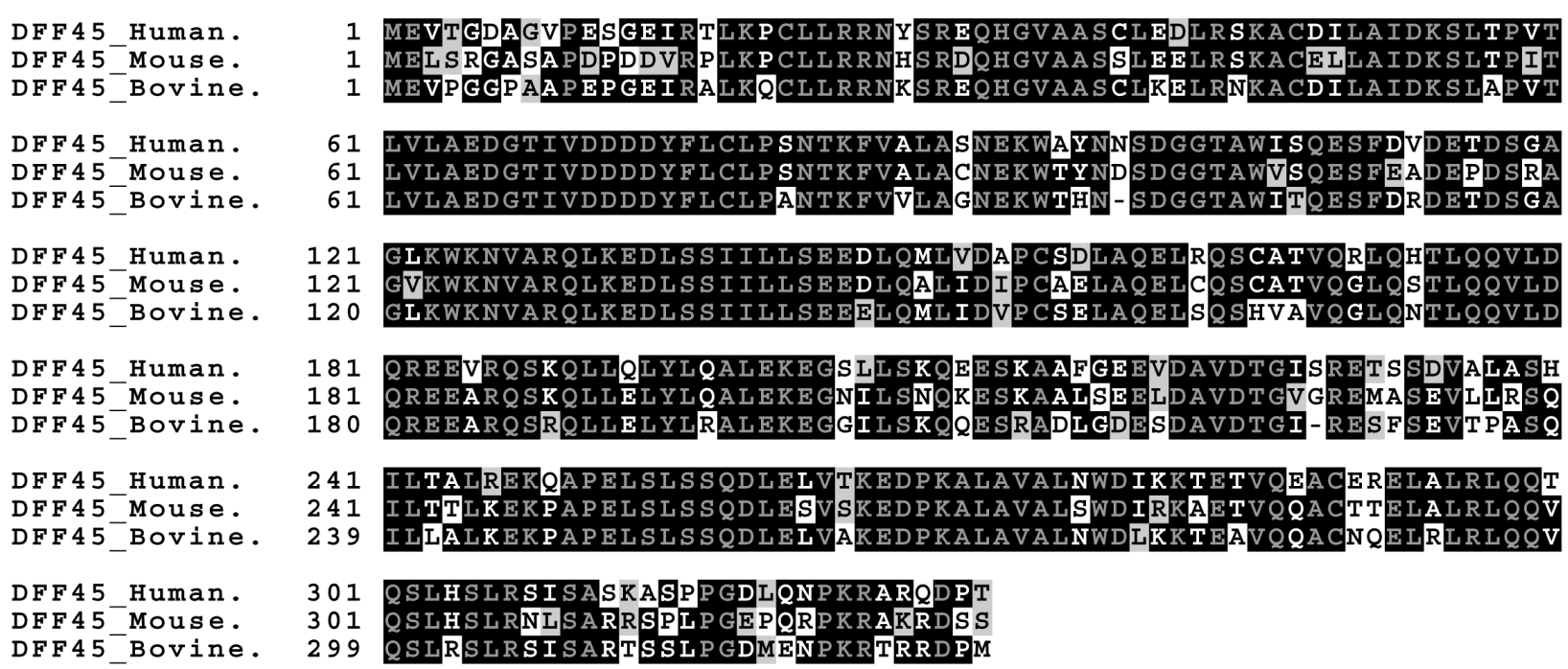

FIG. 21: Sequence alignment of DFF45. Alignment of the amino acid sequences of DFF40 from human, mouse and bovine. Identical amino acids are indicated by a black background and additionally shown in gray when they are conserved in all sequences. Similar amino acids are shaded in gray. A basic stretch resembling the consensus motif of monopartite cNLS is underlined. The numbers indicate the amino acid position in the respective sequence. 
possible that mouse DFF45 is translocated into the nucleus by importin $\alpha / \beta$ via a bipartite cNLS. Further experiments are required to verify whether DFF45 from different species use the same nuclear transport pathway.

\subsection{DFF40 contains a NLS in the C-terminal tail}

The characterization of the individual DFF40 subunit with regard to its nuclear transport capability was done in a similar way as with the monomeric DFF45 subunit. More precisely, the essential role of the C-terminal tail of DFF40 for nuclear uptake of the DFF complex was further elucidated by in vitro binding studies with recombinant transport receptors. The use of C-terminally truncated DFF40 in complex with wild type DFF45 as bait revealed that the C-terminal region (aa 324-338) of DFF40 is required for binding of importin $\alpha / \beta$ to the DFF complex (Fig. 15B and D). Furthermore, caspase-3-activated DFF40 directly interacted with importin $\alpha / \beta$ in GST-pull down assays (Fig. 16A). In addition, fusion of amino acids 301-338 of DFF40 is sufficient for nuclear translocation of a cytoplasmic EGFP-EGFP-GST fusion protein as observed by in vivo transfection experiments in HeLa cells (Fig. 16D). These results together indicate that the C-terminal tail of DFF40 also harbors an independent NLS. The binding of importin $\alpha / \beta$ to active DFF40 was decreased when the importin $\alpha$ minor site mutant $(\alpha \mathrm{E} 388 \mathrm{R})$ was used in pull down assays (Fig. 18) suggesting that the NLS in DFF40 is a classical bipartite type. However, since binding of importin $\alpha \mathrm{E} 388 \mathrm{R} / \beta$ to a control substrate exhibiting a monopartite cNLS was not tested it can not be excluded that the affinity for monopartite cNLS is additionally decreased in this importin $\alpha$ minor site mutant. Leung et al. (2003) analyzed the behavior of the respective minor site mutant of yeast importin $\alpha$ (SRP1p) in binding assays, using fluorescence anisotropy. They observed that binding of this mutant to a monopartite cNLS was about five fold decreased compared to a stronger decrease of about 18-fold for binding to a bipartite cNLS (Leung et al., 2003). The use of importin $\alpha$ E388R however did not affect the binding of importin $\alpha / \beta$ to immobilized monomeric GST-DFF45. Since DFF45 exhibits a monopartite cNLS (see previous chapter), this importin $\alpha$ minor binding site mutant seems to be functional for binding to monopartite cNLSs. Although amino acids 301-338 of DFF40 show no homology to the consensus motif of bipartite cNLSs, lysines 308 and 309 together with the downstream basic cluster RxKRK may form a bipartite cNLS with an extended linker. To examine this possibility K308 and K309 were substituted with alanine. This substitution, however, did neither affect binding of importin $\alpha / \beta$ to activated DFF40 (Fig. 20) nor did it affect the nuclear localization of the EGFP-EGFP-GST-DFF40(aa301-338) fusion protein (data not shown). Hence, these amino acids do not belong to a bipartite cNLS. Further basic amino acids 
that are likely to participate in a potential bipartite cNLS can not be found in the C-terminal tail of DFF40. Although the basic cluster RxKRK in DFF40 is essential for nuclear import of the DFF complex it does not fulfill the consensus motif $\mathrm{K}-\mathrm{K} / \mathrm{R}-\mathrm{X}-\mathrm{K} / \mathrm{R}$ for monopartite cNLSs. Therefore, it is unlikely that this sequence of DFF40 represents a monopartite cNLS. In conclusion, the C-terminal region of DFF40 probably does not contain a cNLS, neither monopartite nor bipartite.

But why then did the C-terminus of DFF40 (aa301-338) fused to the cytoplasmic EGFP-EGFPGST protein accumulate in the nucleus of transfected cells? GST-pull down assays demonstrated that the C-terminus of DFF40 interacts with importin $\beta$ rather than with the importin $\alpha / \beta$ heterodimer (Fig. 16B). Thus, nuclear import of EGFP-EGFP-GST-DFF40(aa301-338) might be mediated by importin $\beta$ instead of importin $\alpha / \beta$. Various proteins can interact directly with importin $\beta$ via their ncNLS. Ribosomal proteins (Jakel and Görlich, 1998), core histones (Baake et al., 2001b) and also importin $\alpha$ (Görlich et al., 1996a) bind to importin $\beta$ with ncNLS highly enriched in basic amino acids. These signals contain more than $30 \%$ of lysines and arginines. Similarly, the C-terminus (aa 301-308) of DFF40 includes a high number of positively charged amino acids (almost 30\%, Fig. 22). As discussed in Kahle et al. (2005) a certain number of basic amino acids in the ncNLS may be required for recognition by the rather acidic importin $\beta$ or other importin $\beta$-family members. Therefore, it is possible that the DFF40 C-terminus resembles a ncNLS. Furthermore, the basic stretch in the C-terminal tail of DFF40 shows similarity to the arginine-rich ncNLS of human immunodeficiency virus type 1 (HIV-1) protein Tat (RKKRRQRRR) (Truant and Cullen, 1999). Tat is transported into the nucleus through direct interaction with importin $\beta$. Although the basic stretch in the C-terminal tail of human DFF40 is not as similar to the Tat NLS as the basic sequence at the very C-terminus of mouse DFF40 (Fig. 22 ) it might represent a recognition motif for importin $\beta$. Full length DFF40 bound importin $\alpha / \beta$ more strongly than its C-terminal tail indicating that other parts of DFF40 contribute to importin $\alpha / \beta$ binding. As mentioned above, cNLS are generally described as short stretches enriched in positively charged amino acids. However, in the meantime cNLSs were characterized that largely differ from the consensus motif for monopartite and bipartite cNLSs. Recently, Rodriguez et al. (2006) reported a discontinuous cNLS in the first 91 amino acids of a cytotoxic variant of human pancreatic ribonuclease. This protein interacts with importin $\alpha$ via lysine 1 , arginines 31-33 and arginines 89-91. Although these basic residues are scattered along the sequence they cluster in the three-dimensional structure, thereby resembling the topological disposition of a bipartite cNLS (Rodriguez et al., 2006). Therefore, a simultaneous interaction of the $\mathrm{C}$-terminus and additional sequences of DFF40 with importin $\alpha / \beta$ might also be possible. 

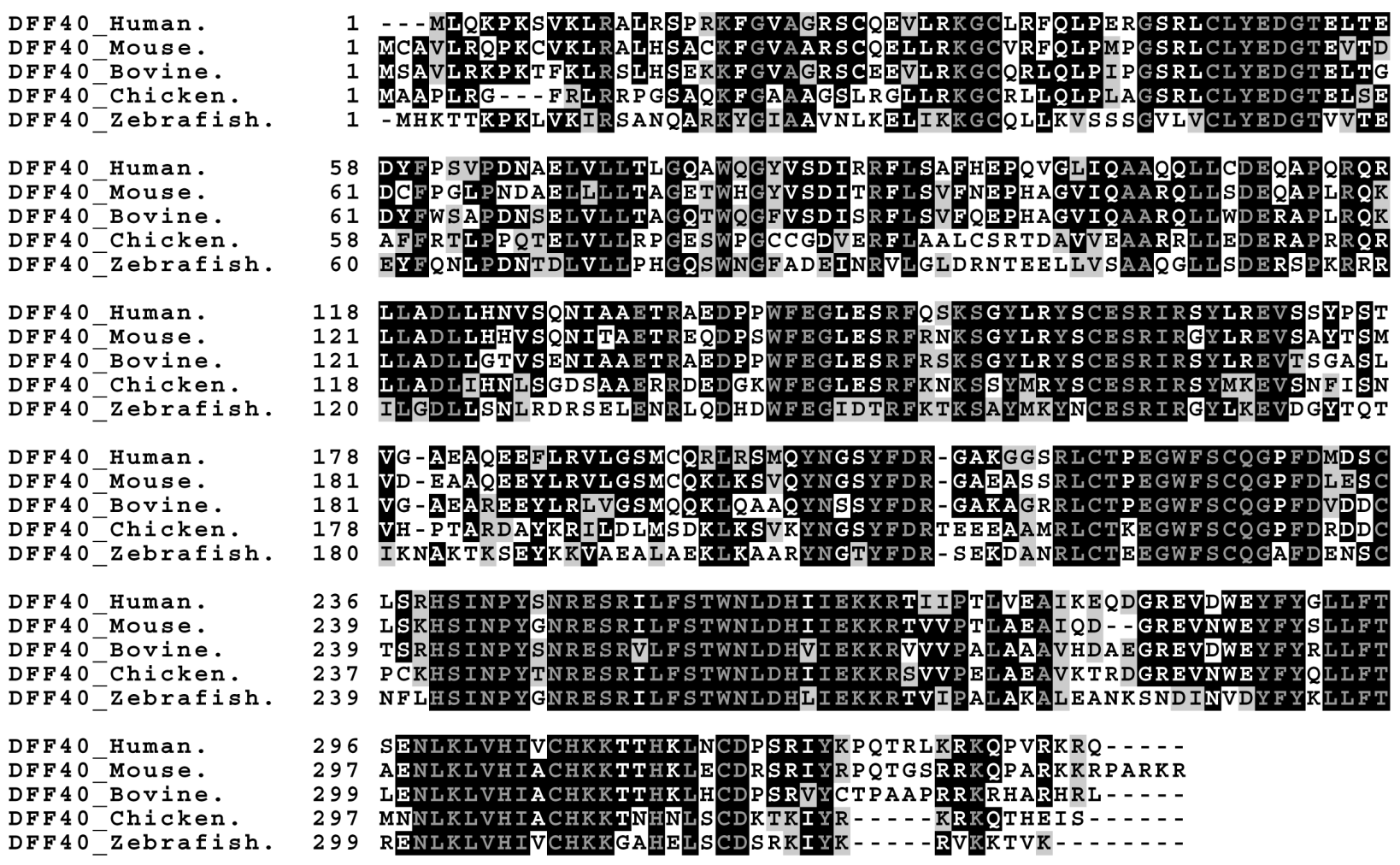

FIG. 22: Sequence alignment of DFF40. Alignment of the amino acid sequences of DFF40 from human, mouse, bovine, chicken and zebrafish . To gain a maximum of homology the sequences were aligned by introducing gaps (-). Identical amino acids are indicated by a black background and additionally shown in gray when they are conserved in all sequences. Similar amino acids are shaded in gray. The numbers indicate the amino acid position in the respective sequence.

Since active DFF40 is probably a homodimer, importin $\alpha / \beta$ could recognize basic amino acids in both DFF40 molecules upon dimerization. However, this is rather unlikely because monomeric GST-DFF40 expressed in the absence of DFF45 was also bound by importin $\alpha / \beta$ and importin $\beta$ to the same extent as caspase-3 activated DFF40 (data not shown). These interactions could not be inhibited by addition of RanGTP, which is probably due to misfolding of bacterially overexpressed DFF40. The fact that importin $\beta$ binds to the C-terminal region of DFF40 and importin $\alpha / \beta$-binding to DFF40 requires this $\mathrm{C}$-terminal region as well suggests that their binding sites in DFF40 partially overlap.

Surprisingly, monomeric, fluorescently tagged, full length DFF40 was not transported into the nucleus in transfected cells (Fig. 16) in contrast to its C-terminal tail fused to EGFP-EGFP-GST as described above. This was unexpected, because active DFF40 interacts with importin $\alpha / \beta$ and importin $\beta$ as mentioned above. Furthermore, the C-terminal region (aa 301-338) of DFF40 proved to be sufficient for nuclear accumulation of a cytoplasmic fusion protein. One explanation for these contradictory observations might be the following: DFF45 is not only 
inhibitor but also chaperone of DFF40 and therefore required for correct protein folding and nuclease activity of DFF40 (Enari et al., 1998; Sakahira et al., 2000). Thus, DFF40 overexpressed in the absence of exogenous DFF45 is most likely misfolded. This of course might (i) hide the ncNLS in the C-terminal region of DFF40 that is otherwise recognized by importin $\beta$ or (ii) destroy the predicted, unusual cNLS in DFF40 that normally binds to importin $\alpha / \beta$. Although endogenous DFF45 partially interacts with overexpressed EGFP-GSTDFF40, as observed in GST-pull down assays (Fig. 10 and 12), nuclear transport of DFF40 can not be observed. In other words, fractions of exogenous DFF40 should have accumulated in the nucleus in complex with endogenous DFF45. As discussed before, endogenous DFF45 can obviously not compensate for coexpression of exogenous DFF45, and can not even partially rescue misfolding of DFF40. Despite the fact that both importin $\alpha / \beta$ and importin $\beta$ interact with active DFF40 in vitro, their role regarding the nuclear import of activated DFF40 remains uncertain. Further experiments are necessary to analyze whether active DFF40 has to be actively transported into the nucleus in vivo. Active nuclear import of DFF40 might perhaps not be necessary when the release of active nuclease occurs after breakdown of the nuclear envelope, considering the timing of apoptotic events. On the other hand, even then importins might be necessary to direct DFF40 to chromatin, similar to intranuclear targeting of the TATA-binding protein by karyopherin 114 (Pemberton et al., 1999). If so, it has to be explored whether importin $\alpha / \beta$, importin $\beta$ or both mediate this process.

Regarding the homology between DFF40 proteins from different species it is remarkable that the very $\mathrm{C}$-terminal region is less well conserved than the entire sequence (Fig. 22). In contrast to the C-terminus of human DFF40 that probably does not contain a cNLS, a basic cluster in the C-terminal tail of each mouse (RKKR), bovine (RRKR), chicken (RKRK) and zebrafish (KRVKK) DFF40 fulfills the consensus motif of monopartite cNLSs (K-K/R-X-K/R). However, further sequence requirements are not satisfied. The C-terminus of mouse DFF40 additionally exhibits a sequence (RR-X 10 -RKR) homologous to the consensus motif for bipartite cNLSs. Hence, the C-terminal regions of mouse, bovine, chicken and zebrafish might contain cNLSs which mediate their nuclear translocation. It would be interesting to analyze these regions in functional terms and with regard to the potential NLSs in human DFF40.

\subsection{Binding models for the interaction between the DFF complex and importin $\alpha / \beta$}

We have demonstrated that nuclear accumulation of the DFF complex requires a basic cluster in each subunit and that monomeric DFF40 and DFF45 contain a functional NLS. In addition, Lechardeur et al. (2000) previously reported that NLSs in the C-terminal regions of DFF40 and 
DFF45 have an additive effect on the nuclear targeting efficiency of the DFF complex. According to the "selective phase" model, the channel of the NPC is filled with a hydrophobic meshwork formed by weakly interacting FG repeats that create the permeability barrier of the NPC (Frey et al., 2006; Ribbeck and Görlich, 2001). Nuclear transport receptors are more hydrophobic than average cytosolic proteins (Ribbeck and Görlich, 2002) and can directly interact with FG repeats (Bayliss et al., 2000b; Fribourg et al., 2001). Thereby, they dissolve the sieve-like structure of the permeability barrier and facilitate the passage of their cargo proteins through the NPC (Ribbeck and Görlich, 2001). However, it was shown that large cargo domains delay transport processes across the nuclear membrane probably because they are also exposed to the hydrophobic phase (Ribbeck and Görlich, 2002). This effect was already detectable for a protein with a molecular weight of $48 \mathrm{kDa}$ whose passage through the NPC was three times slower than that of a $38 \mathrm{kDa}$ protein. The delay in nuclear import can be compensated when more than one transport receptor molecule facilitates the passage. Thus, the solubility in the permeability barrier and thereby the transport efficiency is determined by the receptor-cargo ratio (Ribbeck and Görlich, 2002). Accordingly, the presence of two NLSs in a large protein would lead to a more rapid translocation through the NPC. Therefore, independent interaction of the cNLS in each DFF subunit, DFF40 and DFF45, with importin $\alpha / \beta$ would probably confer efficient nuclear import of the DFF complex. However, the differences in the strength of importin $\alpha / \beta$-binding between the DFF complex and single DFF subunits in GST-pull down assays can not be explained by additive binding of importin $\alpha / \beta$ to both subunits in the DFF complex. For comparison, Fig. 23 summarizes the data regarding the importin $\alpha / \beta$-binding of the DFF complex and its subunits, respectively, mentioned above in the results section. These were obtained from GST-pull down assays with wild type $\operatorname{DFF}(A)$, C-terminally truncated DFF complexes $(B$ and $C$ ), and monomeric DFF subunits $(A)$. Importin $\alpha / \beta$ moderately interacted with active GST-DFF40 (Fig. $23 \mathrm{~A}$ middle panel) while interaction of importin $\alpha / \beta$ with monomeric GST-DFF45 was only very weak (Fig. 23A right panel). Much more GST-DFF45 had to be immobilized to detect importin $\alpha / \beta$-binding. When comparable amounts of immobilized GSTDFF40 and GST-DFF45 were used, binding of importin $\alpha / \beta$ to DFF45 could not be detected (data not shown). In contrast, interaction of importin $\alpha / \beta$ with DFF complexes, namely HisDFF40/GST-DFF45 and GST-DFF40/His-DFF45, was much stronger (Fig. 23A left and middle panel). Furthermore, deletion of the C-terminal tail of either DFF45 (Fig 23B) or DFF40 (Fig. $23 C$ ) almost completely abolished binding of importin $\alpha / \beta$ to the respective truncated DFF complexes. When the DFF complex interacts with importin $\alpha / \beta$ via two independent NLSs in the two DFF subunits one would expect that binding of the C-terminally truncated DFF complex is 
A

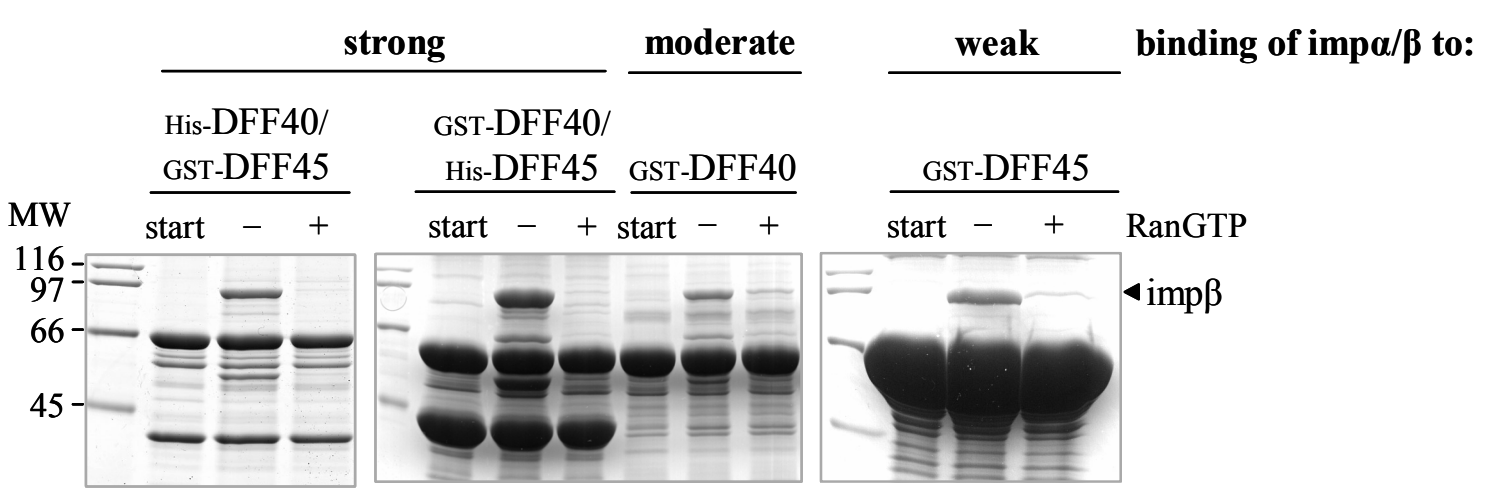

B

weak binding of impa/ $\beta$ to:

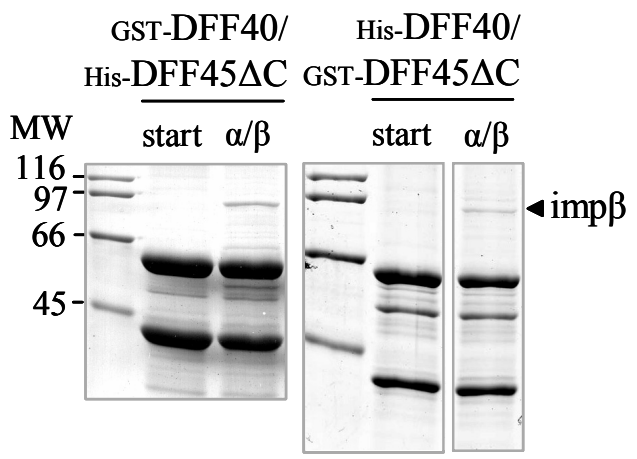

C

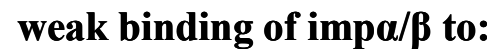

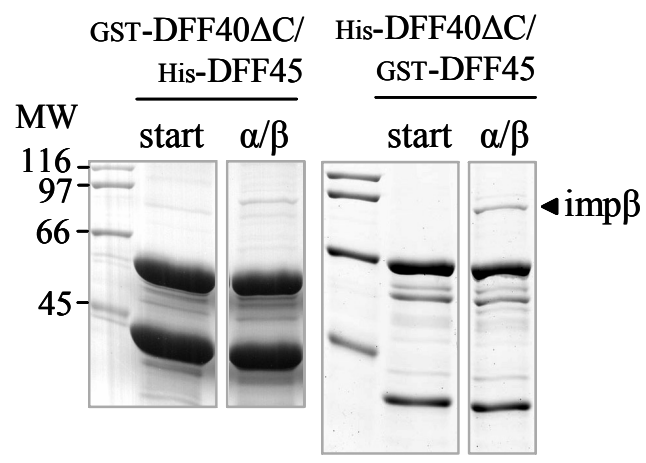

FIG. 23: Binding of importin $\alpha / \beta$ to a NLS unique for the DFF complex. Comparison of the binding of importin $\alpha / \beta$ to the DFF complex, monomeric DFF40, and monomeric DFF45 (compiled from figures 7 and 13-16). Immobilized proteins were incubated with importin $\alpha$ and importin $\beta$ from bacterial lysates in the absence or presence of RanGTP as indicated. Bound fractions were analyzed by SDS-PAGE followed by Coomassie staining. $A$, Importin $\alpha / \beta$ was strongly bound to DFF complexes (left and middle panel). In contrast, importin $\alpha / \beta$ moderately interacted with active GST-DFF40 (middle panel) while interaction with monomeric GST-DFF45 was very weak as much more GST-DFF45 has to be immobilized to detect the binding (right panel). $B$ and $C$, truncation of amino acids 306-331 of DFF45 (DFF45 $\Delta$ C) (B) or of amino acids 324-338 of DFF40 (DFF40 $\Delta$ C) (C) almost completely abolished binding of importin $\alpha / \beta$ to the DFF complex. To simplify the figure only the position of importin $\beta$ on the gel is indicated (see also Fig. 7 and Fig. 13-16). MW, molecular weight in kilodalton; imp, importin.

comparable to importin $\alpha / \beta$-binding of the monomeric subunit that is not truncated in the DFF complex. Conversely, interaction of importin $\alpha / \beta$ with a DFF complex containing C-terminally deleted DFF45, but full length DFF40, was much weaker than the interaction of importin $\alpha / \beta$ with DFF40 alone (Fig. 23A middle panel and B). These observations can not be explained with the existence of two independent NLSs in the DFF complex but indicate a great amount of importin $\alpha / \beta$-binding cooperativity. This cooperativity can be explained by the following model according to Stüven et al. (2003). Instead of independent binding of importin $\alpha / \beta$ to DFF40 and 
DFF45 one importin $\alpha / \beta$ heterodimer simultaneously interacts with both subunits, DFF40 and DFF45. In this scenario, the free energy released upon binding of the DFF40/DFF45 complex would equal the sum of the individual importin $\alpha / \beta$-DFF40 and importin $\alpha / \beta$-DFF45 interactions. According to Gibb's law binding constants increase exponentially with binding energies. Therefore, the DFF-importin $\alpha / \beta$ interaction would be much stronger when one importin $\alpha / \beta$ heterodimer contacts both DFF40 and DFF45 compared to individual interactions between importin $\alpha / \beta$ and the DFF subunits in the DFF complex. This binding model poses the question as to how DFF40 and DFF45 simultaneously interact with importin $\alpha / \beta$. Besides its interaction with importin $\alpha / \beta$ active DFF40 additionally showed binding to importin $\beta$ alone. The interaction of importin $\beta$ with DFF40 is thereby most likely mediated by the basic C-terminal tail of DFF40, representing a ncNLS. In addition, importin $\beta$ is for instance able to interact at the same time with the IBB domain of importin $\alpha$ via its cargo binding site I and with PTHrP via its cargo binding site II (Cingolani et al., 2002). Therefore, a possible interaction mechanism explaining the strong binding of importin $\alpha / \beta$ to the DFF40/DFF45 complex could be as follows: The cNLS of DFF45 binds via importin $\alpha$ using cargo binding site I of importin $\beta$, the C-terminus of DFF40 (ncNLS) simultaneously binds to cargo binding site II of importin $\beta$. To test this model, GSTpull down assays with $\mathrm{N}$-terminal deletion mutants of importin $\beta$ that lack the cargo binding site II were performed. According to the interaction model, the use of N-terminally truncated importin $\beta$ should result in reduced binding of the DFF complex to importin $\alpha / \beta$ due to the lack of importin $\beta$ cargo binding site II. However, binding of N-terminally deleted importin $\beta$ molecules to the DFF complex was not more reduced than to the control substrate nucleoplasmin (Fig. 17). Hence, these results do not support this binding model.

Another possible mechanism for the binding of one importin $\alpha / \beta$ heterodimer to the DFF complex would be the following: Nuclear import of DFF45 is mediated by a monopartite cNLS which does not completely fulfill the sequence requirements for this type of localization signal. Therefore, the monopartite cNLS of DFF45 confers only weak importin $\alpha / \beta$-binding that is not sufficient for nuclear translocation of the DFF complex. In contrast, sequence requirements for bipartite cNLSs are less stringent (Conti and Kuriyan, 2000). Hence, suboptimal interactions between the monopartite cNLS of DFF45 and the importin $\alpha$ major binding site may be tolerated due to additional contacts of basic amino acids in the C-terminus of DFF40 with the minor binding site of importin $\alpha$. The binding studies with the importin $\alpha$ minor binding site mutant ( $\alpha$ E388R) demonstrated that efficient importin $\alpha / \beta$-binding to the DFF complex depends indeed on the minor binding site of importin $\alpha$. Binding of importin $\alpha E 388 \mathrm{R} / \beta$ to the DFF complex and to the control substrate for a bipartite cNLS, nucleoplasmin, was strongly decreased (Fig. 18B 
and $C$ ). These data combined with the fact that the C-terminal regions of both subunits, DFF40 and DFF45, are essential for importin $\alpha / \beta$-binding and nuclear transport strongly support this model. In this scenario, dimerization of the DFF subunits leads to the formation of an intermolecular bipartite cNLS that mediates strong binding of importin $\alpha / \beta$. To further prove this model, a potential negative side-effect of the mutation in the minor binding site of importin $\alpha$ $(\alpha \mathrm{E} 388 \mathrm{R})$ on the interaction with monopartite cNLSs should be analyzed.

Other interaction models can not be excluded. For instance, it is also possible that DFF45 in complex with DFF40 interacts with the minor binding site of importin $\alpha$ while the basic cluster of DFF40 contacts the major binding site of importin $\alpha$ (vice versa to the interaction model described above). Furthermore, GST-pull down assays with active DFF40 and amino acids 314338 of DFF40 indicated that, apart from the C-terminal tail, additional region(s) in DFF40 contribute to the interaction with the importin $\alpha / \beta$ heterodimer. Therefore, these amino acids may also be involved in binding of importin $\alpha / \beta$ to the DFF complex.

The DFF complex is a large protein of about $95 \mathrm{kDa}$ in size or about $190 \mathrm{kDa}$ when it consists of two heterodimers as suggested by Lechardeur et al. (2005). Since large cargo proteins delay import receptor-mediated passage through the nuclear pore (Ribbeck and Görlich, 2001) the large size of the DFF complex may argue against a single bipartite cNLS. However, despite the fact that a higher import receptor-cargo ratio increases the efficiency of translocation through the nuclear pore there are also examples suggesting that one NLS is sufficient to convey translocation of large proteins. Among them are the $82 \mathrm{kDa}$ SV40 large T antigen (Kalderon et al., 1984b), hypoxia inducible factor (HIF) $2 \alpha(96 \mathrm{kDa})$ which contains a potential bipartite cNLS (Luo and Shibuya, 2001), and epidermal growth factor receptor (EGFR) ErbB1 (134 kDa) (Hsu and Hung, 2007). Furthermore, if DFF is a tetrameric complex, according to our binding model, it would contain two intermolecular bipartite cNLSs.

\subsection{Nuclear transport of the DFF complex}

Based on the results of all experiments described in this study, the following model for nuclear transport of the DFF complex is proposed (Fig. 24): Importin $\alpha / \beta$ simultaneously interacts with basic amino acids located in the C-terminal tail of each DFF subunit, DFF40 (RxKRK) and DFF45 (KRxR), leading to nuclear import of the DFF complex. Therefore, DFF45 is not only chaperone and inhibitor of DFF40 but also plays an essential role in nuclear targeting of the nuclease. In addition, DFF45 exhibits an independent monopartite cNLS that also confers importin $\alpha / \beta$ mediated nuclear accumulation. The presence of monomeric DFF45 in the nucleus could result in binding of accidentally activated DFF40 and therefore prevent DNA degradation 


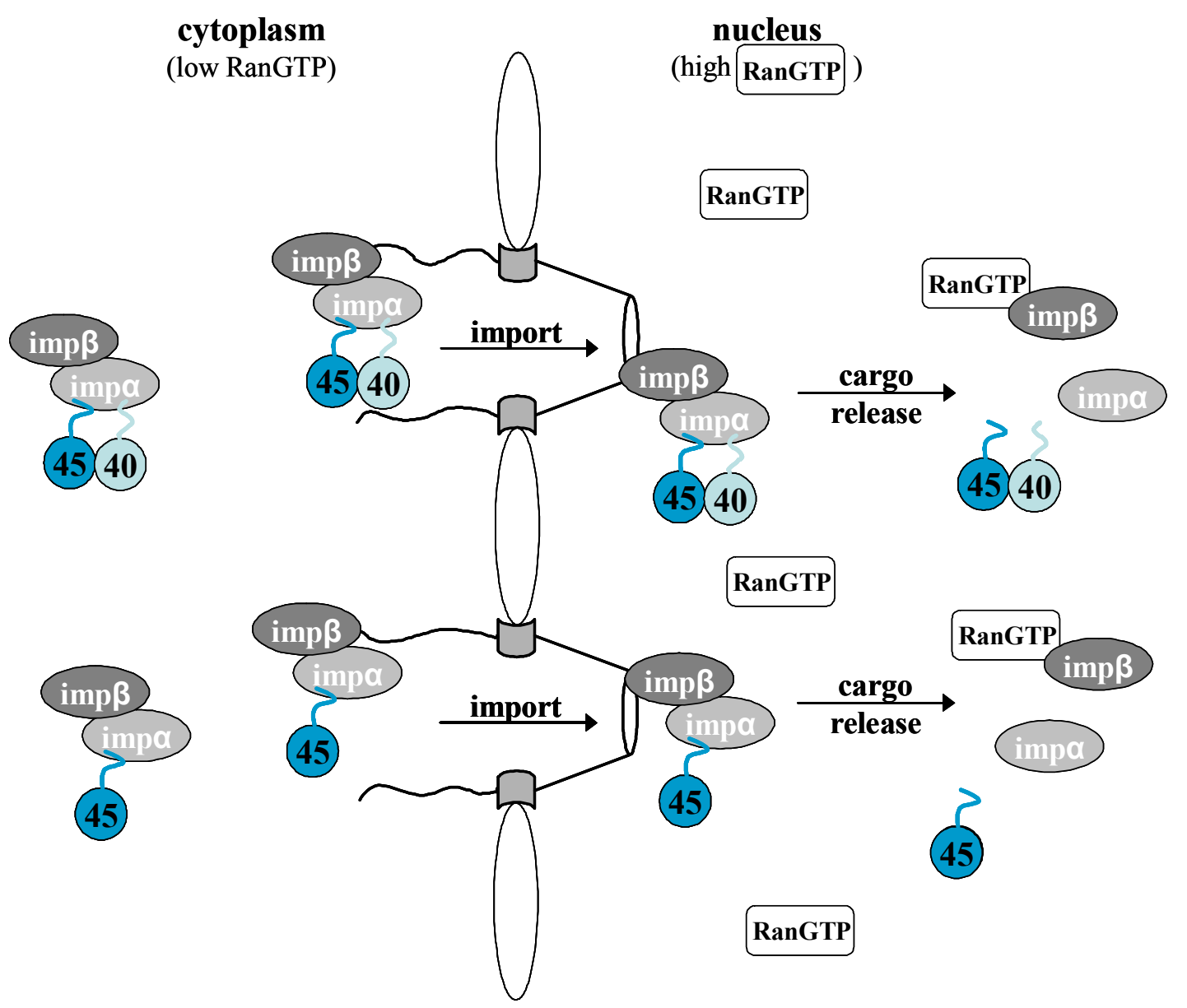

FIG. 24: Model for the nuclear import of human DFF complex and monomeric DFF45. Nuclear transport of the DFF complex and monomeric DFF45 is mediated by the importin $\alpha / \beta$ heterodimer. DFF45 exhibits a monpartite cNLS in its C-terminal region that mediates the interaction with importin $\alpha / \beta$. Furthermore, this cNLS and additional basic amino acid residues in the C-terminal tail of DFF40 form an intermolecular bipartite cNLS in the DFF complex which is recognized by importin $\alpha / \beta$. The monopartite cNLS of DFF45 thereby presumably contacts the major binding site of importin $\alpha$ while the basic cluster in the C-terminus of DFF40 interacts with the minor binding site of importin $\alpha$. The C-terminal regions of the DFF subunits are indicated as lines. [This figure was generated in the style of a figure from (Görlich and Kutay, 1999)]

in healthy cells. DFF35, the shorter isoform of DFF45, lacks the C-terminal region containing the cNLS and is thus restricted to the cytoplasm (Gu et al., 1999; Samejima and Earnshaw, 2000). Since only DFF45 fulfills the function as chaperone of DFF40, DFF35 is also not included in DFF complexes in proliferating cells (Gu et al., 1999; Sakahira et al., 1999). However, DFF35 is able to inhibit the nuclease activity of DFF40 and might therefore fulfill the same "security" role in the cytoplasm as monomeric DFF45 in the nucleus.

Regarding nuclear uptake of a complex due to sequence elements distributed on different subunits, Liku et al. (2005) recently demonstrated that nuclear transport of the Mcm2-7 complex 
is mediated by two potential cNLSs on the Mcm2 and Mcm3 subunit. Mcm2-7 consists of six subunits and is the putative replicative helicase. Each of the two cNLSs is required but not sufficient for nuclear accumulation of the Mcm2-7 complex. Whether both signals are recognized by different import receptors or by the same molecule has still to be elucidated. Complex formation as a prerequisite for nuclear import would prevent nuclear accumulation of Mcm subcomplexes and may therefore protect the genomic DNA for uncontrolled unwinding (Liku et al., 2005). Nuclear transport in dependence of complex assembly could be a general mechanism for the functional regulation of protein complexes. However, both subunits of the DFF complex exhibit independent NLSs. Why is nuclear import of the DFF complex mediated by a unique cNLS instead of import receptor binding to the individual NLSs? One explanation could be that the NLS in DFF40 does not have a functional relevance. In this case, only DFF45 would expose an independent NLS that is sufficient for nuclear targeting of the chaperone monomer. However, because of its weak binding towards importin $\alpha / \beta$ the cNLS in DFF45 alone does not mediate nuclear passage of the DFF complex. The absence of a functional NLS in DFF40 would ensure that the nuclease does enter the nucleus only in complex with its inhibitor DFF45. This has to be elucidated in further experiments. Binding of importin $\alpha / \beta$ and importin $\beta$ to monomeric DFF 40 and the nuclear accumulation of the C-terminal tail of DFF40 fused to EGFP-EGFP-GST do not favor this hypothesis. On the other hand, these data do not exclude this possibility either.

\subsection{Activation of DFF40 in the nucleus requires nuclear entry of caspase-3}

Upon induction of apoptosis, DFF40 is activated through caspase-3 mediated cleavage of DFF45 (Liu et al., 1999; Sakahira et al., 1998). Therefore, caspase-3 has to cross the nuclear membrane as well. Whether this occurs prior to apoptosis in its inactive form (procaspase-3) or after apoptosis induction in its active form is still not clear and the mechanism of caspase-3 translocation into the nucleus is still under discussion (see chapter 1.3). Nevertheless, already a decade ago Yasuhara et al. (1997) demonstrated that apoptotic nuclear changes promoted by active caspase-3 were prevented by addition of WGA. Since WGA treatment blocks access to nucleoporins and thereby inhibits active nuclear transport processes it could be concluded that caspase-3 does not passively diffuse into the nucleus. The majority of active nuclear transport processes are mediated by members of the $\beta$-family transport receptors and therefore caspase- 3 might interact with importins. However, in GST-pull down assays with immobilized procaspase-3 we did not observe binding of importin $\alpha / \beta$, importin $\beta$, transportin, importin 5 , importin 7, importin 9 or importin 13 (unpublished results). Furthermore, procaspase-3 did not 
accumulate in the nuclei of digitonin-permeabilized HeLa cells in the presence of reticulocyte lysate. These preliminary results indicated that procaspase- 3 is not translocated into the nucleus by import receptors. In addition, the cytoplasmic localization of procaspase- 3 in the presence of reticulocyte lysate makes a direct interaction of procaspase-3 with nucleoporins very unlikely. Nevertheless, these results do not rule out that caspase-3 is transported into the nucleus only in its active form. Indeed, in the meantime Kamada and colleagues proposed that caspase-3 is actively transported into the nucleus mediated by a substrate-like protein (Kamada et al., 2005a; Kamada et al., 2005b). A-Kinase-Anchoring Protein (AKAP) 95 interacts with active caspase-3 via a tetrapeptide that is related to the caspase- 3 recognition site but lacks the cleavage site aspartate. Therefore, AKAP 95 is not cleaved by caspase-3 (Kamada et al., 2005a). AKAP 95 contains a putative bipartite cNLS and colocalized with caspase-3 in the nucleus of apoptotic cells. Since downregulation of AKAP 95 and mutation in the putative cNLS reduced apoptotic nuclear morphology changes, AKAP 95 is a potential carrier protein for active caspase-3 (Kamada et al., 2005a). Although interaction was additionally observed between AKAP 95 and procaspase-3 the precursor was not detected in the nucleus. These data suggest that active caspase-3 enters the nucleus upon apoptosis induction where it cleaves DFF45 and other nuclear substrates.

\subsection{Outlook}

The participation of different subunits in transport receptor recognition could be a powerful tool for regulating the subcellular localization of protein complexes. Such a mechanism was recently proposed by Liku et al. (2005) for the Mcm2-7 complex and may also account for other protein complexes. However, it is probably difficult to identify those signals in cases where they do not resemble cNLS.

Regarding the interaction between the DFF complex and importin $\alpha / \beta$ further experiments are required to finally prove that nuclear transport is mediated by an intermolecular cNLS. A potent approach would be the exact measurement of the molecular weight of the DFF complex and the importin $\alpha / \beta$-DFF complex, for instance by light scattering. Firstly, this method could be used to examine the subunit composition of the DFF complex thereby discriminating between a heterodimeric and heterotetrameric structure. Secondly, determination of the molecular weight of the importin $\alpha / \beta$-DFF complex would uncover the number of importin $\alpha / \beta$ molecules bound to the DFF complex. The interaction of one importin $\alpha / \beta$ molecule with a DFF40/DFF45 heterodimer would support importin $\alpha / \beta$-binding to an intermolecular bipartite cNLS while two importin $\alpha / \beta$ molecules would point to the recognition of one independent NLS in each subunit. 
In addition, crystallographic analysis would reveal the binding mechanism of importin $\alpha / \beta$ to the DFF complex in an atomic resolution. Thereby particular basic amino acids in the C-terminal regions of the DFF subunits interacting with the major and minor binding site of importin $\alpha / \beta$ could be identified.

In this context, it is necessary to examine whether active DFF40 is transported into the nucleus or not. For that purpose, DFF40 has to be coexpressed with DFF45 first and then activated through caspase-3 mediated cleavage of DFF45 to generate properly folded DFF40 (as done in this study). The general behaviour of DFF40 regarding nuclear transport could then be examined with fluorescently labeled active DFF40, either microinjected in the cytoplasm of HeLa cells or used in in vitro nuclear import assays. If active DFF40 is imported into nuclei of permeabilized cells, in vitro nuclear import assays could be further used to analyze whether importin $\alpha / \beta$ or importin $\beta$ mediate nuclear transport.

Since the monopartite cNLS of DFF45 is also involved in importin $\alpha / \beta$-binding to the DFF complex kinetics between nuclear accumulation of the DFF complex and the monomeric DFF45 could be compared. For this purpose, microinjection studies in Xenopus leavis oocytes could be perfomed. After injection of the DFF complex or monomeric DFF45 into the cytoplasm of the oocytes the amount of nuclear DFF complex or DFF45 would be detected at distinct time points. Finally, based on the differences in the C-terminal tails of DFF40 from different species it would be appealing to analyze whether the nuclear transport pathway of the DFF complex is conserved among species. Especially, nuclear entry of the mouse DFF complex could be different because both subunits, DFF40 and DFF45, contain basic stretches in their C-terminal regions that resemble cNLSs, either monopartite or bipartite. 


\section{Material and Methods}

\subsection{Materials}

\subsubsection{Organisms}

\subsubsection{Bacterial strains}

The following Escherichia coli (E. coli) strains have been used:

$\underline{\text { Cloning strains }}$

$\underline{\mathrm{DH} 5 \alpha}$ (Clontech, Heidelberg)

Genotype: $\quad \operatorname{rec} \mathrm{A}^{-}, \mathrm{F}^{-}$, end $\mathrm{A} 1, \operatorname{gyr} \mathrm{A} 96$, thi- $1, h s d \mathrm{R} 17\left(\mathrm{r}_{\mathrm{k}}{ }^{-} \mathrm{m}_{\mathrm{k}}{ }^{+}\right)$, sup44, relA1

Top10 (Invitrogen, Karlsruhe)

Genotype: $\quad \mathrm{F}^{-}$mcrA $\Delta(m r r-h s d \mathrm{RMS}-m c r \mathrm{BC})$ (80lacZ $\Delta \mathrm{M} 15 \Delta$ lacX74 recA1 ara 139 $\Delta($ ara-leu $) 7697$ galU galK rpsL $\left(\mathrm{Str}^{\mathrm{R}}\right)$ end $\mathrm{A} 1$ nup $\mathrm{G}$

\section{Protein expression strains}

BL21 (DE3) (Stratagene, Heidelberg)

Genotype: $\quad \mathrm{F}^{-}$ompT $h s d S_{B}\left(r_{B}^{-} m_{B}^{-}\right)$gal dcm (DE3)

$\underline{\mathrm{JM} 109}$ (Stratagene, Heidelberg)

Genotype: $\quad$ endA1 recA1 gyrA96 thi-1 hsdR $17\left(\mathrm{r}_{\mathrm{K}}{ }^{-} \mathrm{m}_{\mathrm{K}}{ }^{+}\right)$relA1 supE44 $\Delta($ lac-proAB $)$

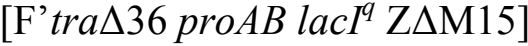

\subsubsection{Cell lines}

$\underline{\text { HeLa cells }}$ were obtained from the German Collection of Microorganisms and Cell Cultures (Deutsche Sammlung von Mikroorganismen und Zellkulturen, Braunschweig; DSMZ Nr. ACC57). HeLa cells are human cervix carcinoma cells which grow epithelial-like in monolayers.

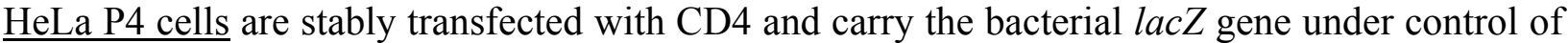
the HIV-1 LTR (Charneau et al., 1994).

\subsubsection{Chemicals}

$\beta$-mercaptoethanol (BME) (Serva, Heidelberg)

Acetic acid (Roth, Karlsruhe)

Acetone (Roth, Karlsruhe)

Acrylamide solution [30\% (w/v) acrylamide, $0.8 \%(\mathrm{w} / \mathrm{v})$ bisacrylamide] (Roth, Karlsruhe)

Adenine (Sigma, Steinheim)

Adenosine-5'-diphosphate (ADP) (Sigma, Steinheim) 
Adenosine-5' -triphosphate (ATP) (Sigma, Steinheim)

Agarose peqGold Universal (Peqlab, Erlangen)

Ammonium acetate (Merck, Darmstadt)

Ammonium chloride (Merck, Darmstadt)

Ammonium peroxodisulfate (APS) (Merck, Darmstadt)

Apyrase (Sigma, Steinheim)

Arginine (Fluka, Taufkirchen)

L-Aspartic acid (Serva, Heidelberg)

Bacto-agar (AppliChem, Darmstadt)

Bacto-peptone (DIFCO, Detroit)

Bacto-tryptone (AppliChem, Darmstadt)

Bacto-yeast extract (AppliChem, Darmstadt)

Boric acid (Roth, Karlsruhe)

Bovine serum albumin (BSA) (Sigma, Steinheim)

Bromophenol blue (Serva, Heidelberg)

$\mathrm{CaCl}_{2}$ (Merck, Darmstadt)

Coomassie Brilliant Blue G-250 (Merck, Darmstadt)

Coomassie Brilliant Blue R-250 (Merck, Darmstadt)

Creatine phosphokinase (Sigma, Steinheim)

Deoxyribonucleoside triphosphates (dNTPs) (Roche, Mannheim)

Diamino-2-phenylindol (DAPI) (Sigma, Steinheim)

Digitonin (Calbiochem, Bad Soden)

Dithiothreitol (DTT) (Biomol, Hamburg)

Dimethylsulfoxide (DMSO) (Sigma, Steinheim)

EDTA (disodium ethylenediaminetetraacetate) (Serva, Heidelberg)

EGTA (ethylene-bis(oxyethylenenitrilo)tetraacetic acid) (Serva, Heidelberg)

Ethanol (Merck, Darmstadt)

Ethidium bromide (Sigma, Steinheim)

Fetal bovine serum (FBS) (Biochrom, Berlin)

Fluorescein isothiocyanate (FITC) (Sigma, Steinheim)

Fluorescein-5-maleimide (Pierce, Rockford, USA)

FLUOS (carboxy fluorescein $N$-hydroxysuccinimide ester) (Roche, Mannheim)

Glycerol (Serva, Heidelberg)

Glycine (Serva, Heidelberg) 
Guanosine-5'-diphoshate (GDP) (Sigma, Steinheim)

Guanosine-5'-triphoshate (GTP) (Sigma, Steinheim)

$\mathbf{H}_{2} \mathrm{O}$ (HPLC purity) (Merck, Darmstadt)

$\mathrm{H}_{2} \mathrm{SO}_{4}$ (Merck, Darmstadt)

$\mathrm{HCl}$ (Merck, Darmstadt)

HEPES ( $N$-2-hydroxyethylpiperazine- $N$ '-2-ethanesulfonic acid) (Serva, Heidelberg)

Histidin (Fluka, Taufkirchen)

Histogel (Vector Laboratories, Burlingame, USA)

Hoechst33258 (Invitrogen, Karlsruhe)

IGEPAL (Sigma, Steinheim)

Imidazole (Roth, Karlsruhe)

IPTG (isopropylthio-ß3-D-galactoside) (Peqlab, Erlangen)

L-Isoleucine (Fluka, Taufkirchen)

Isopropanol (Merck, Darmstadt)

$\mathbf{K}_{2} \mathrm{HPO}_{4}$ (Merck, Darmstadt)

$\mathrm{KCl}$ (Merck, Darmstadt)

$\mathrm{KH}_{2} \mathrm{PO}_{4}$ (Merck, Darmstadt)

LiCl (Serva, Heidelberg)

L-Lysin-monohydrochloride (Fluka, Taufkirchen)

Lysozyme (Sigma, Steinheim)

Magnesium acetate (Serva, Heidelberg)

MEM (Modified Eagle's Medium) (Biochrom, Berlin)

Methanol (Serva, Heidelberg)

L-Methionin (Fluka, Taufkirchen)

$\mathrm{MgCl}_{2}$ (Merck, Darmstadt)

Milk powder (Roth, Karlsruhe)

$\mathrm{Na}_{2} \mathrm{HPO}_{4}$ (AppliChem, Darmstadt)

$\mathrm{NaH}_{2} \mathrm{PO}_{4}$ (Merck, Darmstadt)

$\mathrm{NaHCO}_{3}$ (Merck, Darmstadt)

$\mathrm{NaOH}$ (Merck, Darmstadt)

Paraformaldehyde (Serva, Heidelberg)

L-Phenylalanine (Fluka, Taufkirchen)

Phosphocreatine (Sigma, Steinheim)

Phosphoric acid (Merck, Darmstadt) 
Ponceau S solution (AppliChem, Darmstadt)

Potassium acetate (Serva, Heidelberg)

L-Prolin (Roth, Karlsruhe)

Reticulocyte lysate (Promega, Mannheim)

SDS (sodium dodecyl sulfate) (Serva, Heidelberg)

L-Serin (Serva, Heidelberg)

Sodium acetate (Merck, Darmstadt)

Sodium azide $\left(\mathrm{NaN}_{3}\right)$ (Roche, Mannheim)

TEMED (N,N,N',N'-tetramethylethylenediamine) (Serva, Heidelberg)

L-Threonine (Serva, Heidelberg)

Trichloroacetic acid (TCA) (Merck, Darmstadt)

Tris [Tris(hydroxymethyl)aminomethane] (Roth, Karlsruhe)

TRITC (Tetramethylrhodamine isothiocyanate) (Sigma, Steinheim)

Triton X-100 (Merck, Darmstadt)

L-Tryptophane (Fluka, Taufkirchen)

Tween-20 (Sigma, Steinheim)

L-Tyrosin (Serva, Heidelberg)

L-Uracil (Fluka, Taufkirchen)

L-Valine (Fluka, Taufkirchen)

Vectashield (Mounting medium with DAPI) (Vector Laboratories, Burlingame, USA)

Wheat germ agglutinin (WGA) (Sigma, Steinheim)

Yeast nitrogen base (DIFCO, Detroit)

\subsubsection{Enzymes}

Alkaline Phosphatase, calf intestinal (CIP) (1 U/ $\mu$ l) (Roche, Mannheim)

Lysozyme (Muramidase), stock solution $50 \mathrm{mg} / \mathrm{ml} \mathrm{H}{ }_{2} \mathrm{O}$ (Sigma, Steinheim)

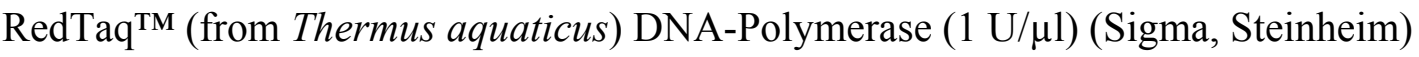

Restriction enzymes (New England Biolabs, Schwalbach; MBI Fermentas, St.Leon-Rot)

RNase A, stock solution $10 \mathrm{mg} / \mathrm{ml}$ (Sigma, Steinheim)

T4 DNA-Ligase (1 U/ $\mu$ 1) (New England Biolab, Schwalbach)

T4 polynucleotide kinase (10 U/ $\mu 1)$ (New England Biolabs, Schwalbach)

Trypsin (Biochrom, Berlin)

Vent $_{\mathrm{R}}{ }^{\circledR}$ (from Thermococcus litoralis) DNA-Polymerase (2 U/ $\left.\mu \mathrm{l}\right)$ (New England BioLabs, Schwalbach) 


\subsubsection{Antibodies}

The following antibodies were used for the detection of immobilized proteins after Western blotting.

Table 1: Antibodies used in this study

\begin{tabular}{|l|l|l|l|}
\hline Antibody & Organism & Company & Dilution \\
\hline anti-DFF40 & rabbit & ProSci Incorporated & $1: 1000$ \\
\hline anti-DFF45/35(NT) & rabbit & ProSci Incorporated & $1: 1000$ \\
\hline anti-GST (B-14): sc-138 & mouse & Santa Cruz Biotechnology & $1: 2000$ \\
\hline anti-GFP (FL): sc-8334 & rabbit & Santa Cruz Biotechnology & $1: 500$ \\
\hline anti-RFP & rabbit & Molecular Biotechnology & $1: 1000$ \\
\hline anti-rabbit-IgG-HRP & goat & Sigma & $1: 100000$ \\
\hline anti-mouse-IgG-HRP & goat & Sigma & $1: 12500$ \\
\hline
\end{tabular}

\subsubsection{Protease inhibitors}

Aprotinin (Roche, Mannheim)

Complete ${ }^{\circledR}$ (EDTA free) protease inhibitor cocktail (pills) (Roche, Mannheim)

Leupeptin (Roche, Mannheim)

Pepstatin (Roche, Mannheim)

Phenylmethylsulfonyl fluoride (PMSF) (Serva, Heidelberg)

\subsubsection{Kits}

Big Dye ${ }^{\mathrm{TM}}$ Terminator Sequencing Ready Reaction (Applied Biosystems, Foster City, USA)

$\mathrm{ECL}^{\mathrm{TM}}$ Western Blotting Detection Reagents (GE Healthcare, München)

Effectene $^{\mathrm{TM}}$ Transfection Reagent (Qiagen, Hilden)

E.Z.N.A Cycle-Pure Kit (Peqlab, Erlangen)

E.Z.N.A. Plasmid-Miniprep Kit II (Peqlab, Erlangen)

NucleoSpin ${ }^{\circledR}$ Plasmid (Macherey-Nagel, Düren)

QIAEX® II Agarose Gel Extraction Kit (Qiagen, Hilden)

Qiagen Plasmid-Maxi/Midi-Kit (Qiagen, Hilden)

$\mathrm{T}_{\mathrm{N}} \mathrm{T}$ coupled reticulocyte lysate system (Promega, Mannheim) 


\subsubsection{Affinity matrices}

Amylose Resin (New England Biolabs, Schwalbach)

Glutathione Sepharose CL 4B (GE Healthcare, München)

GST Bind Resin (Novagen - Merck Biosciences, Bad Soden)

Nickel-NTA Agarose (Qiagen, Hilden)

\subsubsection{Chromatography columns}

NAPTM-5 column (GE Healthcare, München)

Mono S column (GE Healthcare, München)

HiLoad Q Sepharose Fast Flow column (GE Healthcare, München)

Superdex® 200 HiLoad 16/60 column (GE Healthcare, München)

Superdex® 200 10/300 GL column (GE Healthcare, München)

Superdex ${ }^{\circledR} 75$ HiLoad 16/60 column (GE Healthcare, München)

Vivaspin (Vivascience; Biofiltronics, Nörten-Hardenberg)

\subsubsection{Filters and membranes}

Folded filters (Ø12.5 cm) (Macherey-Nagel, Düren)

Filters 2668 (Whatman, Dassel)

Syringe filters $(0.2 \mu \mathrm{m})$ (Roth, Karlsruhe)

OPTITRAN BA-S 83 (nitrocellulose membrane) (Whatman, Dassel)

Whatman 3MM (Whatman, Dassel)

ZelluTrans (dialysis membrane) (Roth, Karlsruhe)

\subsubsection{Molecular weight standards}

DNA

$\lambda$-DNA size markers (digested HindIII or EcoRI-HindIII) (MBI Fermentas, St.Leon-Rot)

Protein

PageRuler ${ }^{\mathrm{TM}}$ Prestained Protein Ladder (Fermentas, St.Leon-Rot)

SDS-PAGE Molecular weight standard broad range marker (BioRad, München)

\subsubsection{Liquid media and solutions}

The following solutions were prepared with deionized water and were sterilized by autoclaving for $20 \mathrm{~min}$ at $121^{\circ} \mathrm{C}$. Solutions containing heat-sensitive substances were sterilized by filtration through a 0.2 micron filter (pore size $0.2 \mu \mathrm{m}$ ). 


\subsubsection{Liquid media and agar plates}

\section{Media for prokaryotes}

Luria-Bertani (LB) medium: $\quad 1 \%(\mathrm{w} / \mathrm{v})$ bacto-tryptone, $0.5 \%(\mathrm{w} / \mathrm{v})$ bacto-yeast extract, $1 \%$ (w/v) $\mathrm{NaCl}, \mathrm{pH} 7.0$

LB agar plates:

$1.5 \%(\mathrm{w} / \mathrm{v})$ bacto-agar in LB medium

SOB medium:

$2 \%(\mathrm{w} / \mathrm{v})$ bacto-tryptone, $0.5 \%(\mathrm{w} / \mathrm{v})$ yeast extract, $0.05 \%(\mathrm{w} / \mathrm{v})$ $\mathrm{NaCl}, 0.019 \%$ (w/v) $\mathrm{KCl}, 10 \mathrm{mM} \mathrm{MgCl} 2$

2xYT medium:

$0.5 \%(\mathrm{w} / \mathrm{v}) \mathrm{NaCl}, 1.6 \%(\mathrm{w} / \mathrm{v})$ bacto-tryptone, $1 \%(\mathrm{w} / \mathrm{v})$ bactoyeast extract

$\underline{\text { Yeast media }}$

Amino acid mix -Leu (10x): $200 \mathrm{mg} / \mathrm{l}$ adenine, arginine, histidin, methionin, proline, tryptophane, uracil; $300 \mathrm{mg} / 1$ isoleucin, lysin, tyrosin; $500 \mathrm{mg} / 1$ phenylalanine; $1000 \mathrm{mg} / \mathrm{l}$ aspartic acid, glutamic acid, serin, valine, threonine; store at $-20^{\circ} \mathrm{C}$

SCD-Leu medium: $1 \mathrm{x}$ amino acid mix $-\mathrm{Leu}, 0.67 \%(\mathrm{w} / \mathrm{v})$ yeast nitrogen base, $4 \%$ (w/v) glucose, $\mathrm{pH} 6.5$

SCGL-Leu medium: $1 \mathrm{x}$ amino acid mix -Leu, 0,67\% (w/v) yeast nitrogen base, $0.1 \%$ $(\mathrm{w} / \mathrm{v})$ glucose, 3\% (v/v) glycerol, 2\% (v/v) lactic acid pH 6.0

YPGLA medium: $1 \%(\mathrm{w} / \mathrm{v})$ bacto-yeast extract, $2 \%(\mathrm{w} / \mathrm{v})$ bacto-peptone, $0.2 \%$ (w/v) glucose, 2\% (v/v) lactic acid, 0.002\% (w/v) adenine, $\mathrm{pH}$ $5-6$

Media for mammalian cells MEM: 10.4 g/1 MEM-powder, 2.38 g/l HEPES, 2.2 g/1 NaHCO 3 , 10\% FBS

DMEM:

DMEM liquid (Invitrogen, Karlsruhe), $2 \mathrm{mM}$ glutamine, 10\% FBS

The media were sterilized by filtration and stored at $4^{\circ} \mathrm{C}$.

\subsubsection{Frequently used buffers and solutions}

DNA gel loading buffer $(5 \mathrm{x})$

$40 \%(\mathrm{w} / \mathrm{v})$ sucrose (in water), $0.1 \%(\mathrm{w} / \mathrm{v})$ bromophenol blue, $0.1 \mathrm{M}$ EDTA (pH 7.5) 
"Laemmli" buffer $(5 \mathrm{x})$

0.25 M Tris, $10 \mathrm{mM}$ EDTA, 1.9 M glycine, $\mathrm{pH}$ 8.7, $0.5 \%$ (w/v) SDS

$\underline{\operatorname{PBS}(10 \mathrm{x})}$

1.4 M NaCl, $27 \mathrm{mM} \mathrm{KCl,} 15 \mathrm{mM} \mathrm{KH}_{2} \mathrm{PO}_{4}, 90 \mathrm{mM} \mathrm{Na}_{2} \mathrm{HPO}_{4}(\mathrm{pH} 7.4)$

$\underline{\text { SDS-PAGE loading buffer }(3 \mathrm{x})}$

(“Sample buffer”): 150 mM Tris- $\mathrm{HCl}$ (pH 6.8), $3 \%$ (w/v) SDS, $3 \%$ (v/v) BME, $30 \%$ (w/v) glycerol, $0.003 \%(\mathrm{w} / \mathrm{v})$ bromophenol blue

TBE buffer $(10 \mathrm{x})$

0.9 M Tris base ( $\mathrm{pH} 8.3$ ), 0.9 M boric acid, 0.025 M EDTA

$\underline{\operatorname{TBS}(10 \mathrm{x})}$

$200 \mathrm{mM}$ Tris- $\mathrm{HCl}$ (pH 7.6), $140 \mathrm{mM} \mathrm{NaCl}$

$\underline{\operatorname{TBST}(10 \mathrm{x})}$

200 mM Tris- $\mathrm{HCl}$ (pH 7.6), $140 \mathrm{mM} \mathrm{NaCl}, 0.1 \%$ Tween 20

TE buffer

$10 \mathrm{mM}$ Tris- $\mathrm{HCl}(\mathrm{pH} 8.0), 1$ mM EDTA (pH 8.0)

TELT buffer

$50 \mathrm{mM}$ Tris- $\mathrm{HCl}$ (pH 7.5), $62.5 \mathrm{mM}$ EDTA, $2.5 \mathrm{M} \mathrm{LiCl,} 4 \%$ (v/v) Triton X-100

Transport buffer

$20 \mathrm{mM}$ HEPES-KOH (pH 7.4), $110 \mathrm{mM}$ potassium acetate, $5 \mathrm{mM}$ magnesium acetate, $0.5 \mathrm{mM}$ EGTA, 2 mM DTT

\subsubsection{Antibiotics}

applied to prokaryotic media

Ampicillin (Binotal®) (Ratiopharm, Ulm): $\quad$ Stock solution: $100 \mathrm{mg} / \mathrm{ml}$ in $\mathrm{H}_{2} \mathrm{O}$

Kanamycin sulfate (Serva, Heidelberg):

Working concentration: $100 \mu \mathrm{g} / \mathrm{ml}$

Stock solution: $10 \mathrm{mg} / \mathrm{ml}$ in $\mathrm{H}_{2} \mathrm{O}$

Working concentration: $50 \mu \mathrm{g} / \mathrm{ml}$

applied to eukaryotic media

Gentamycin (PAA, Cölbe):

Stock solution: $10 \mathrm{mg} / \mathrm{ml}$ in $\mathrm{H}_{2} \mathrm{O}$

Working concentration: $50 \mu \mathrm{g} / \mathrm{ml}$

Penicillin-Streptomycin (Biochrom, Berlin): $\quad$ Stock solution: $10 \mathrm{mg} / \mathrm{ml}$

Working concentration: $1 \%(\mathrm{v} / \mathrm{v})$ 


\subsubsection{Radioactive isotopes}

L- $\left[{ }^{35} \mathrm{~S}\right]$-methionine (Amersham Biosciences, Little Chalfont, UK)

$10 \mu \mathrm{Ci} / \mathrm{ml}, 1000 \mathrm{Ci} / \mathrm{mmol}$ (specific radioactivity)

\subsubsection{Vectors}

pGEM®-T-Easy (Promega, Mannheim)

pGEX4T-1 (GE Healthcare, München)

pEGFP-C1 (Clontech, Heidelberg)

pET-41a(+) (Novagen - Merck Biosciences, Bad Soden)

pET-21b(+) (Novagen - Merck Biosciences, Bad Soden)

pMal-c2x (New England Biolabs, Schwalbach)

pQE30 (Qiagen, Hilden)

\subsubsection{Oligonucleotides}

The oligonucleotides used in this study were synthesized by MWG-Biotech (Ebersberg) and by Sigma (Steinheim).

\subsubsection{Hardware}

ÄktaPurifier-System (GE Healthcare, München)

Biofuge pico (Heraeus, Hanau)

Cell-counter Casy Counter (Schärfe System, Reutlingen)

$\mathrm{CO}_{2}$-Inkubator Heraeus BBD 6220 (Kendro, Langenselbold)

Digital Sonifier (Branson Sonifier, Schwäbisch Gmünd)

Fluorescence microscope Axioskop 20 with AxioCam MRm (Zeiss, Oberkochen)

GeneAmp PCR system 2400 (Perkin Elmer, Freyung)

Horizontal laminar flow hood HERAsafe Typ 18/2 (Heraeus, Hanau)

Incubator cytoperm 2 (Heraeus, Hanau)

Incubator Modell CO 24 (New Brunswick, Edison, USA)

Incubator Shaker Modell G 25 (Scientific Co. Inc, New Jersey, USA)

Incubator Typ B5050 (Heraeus, Hanau)

Inverse microscope Axiovert 25 (Zeiss, Oberkochen)

Liquid nitrogen storage container (Air Liquide Kryotechnik, Düsseldorf)

Magnetic stirrer IKAMAG® RET (Janke \& Kunkel, Staufen)

Mini-gel-chambers (Biometra, Göttingen; workshop) 
pH-Meter CG820 (Schott, Mainz)

Photometer ULTROSPEC 3000 (GE Healthcare, München)

Power supply Power Pack P25 (Biometra, Göttingen)

Sorvall RC 5B Plus (Kendro, Langenselbold)

Speed Vac SC 100 (Savant, Holbrook, NY, USA)

T3 Thermocycler (Biometra, Göttingen)

Table centrifuge 5415 (Eppendorf, Hamburg)

Varifuge 3.0 R (Heraeus, Hanau)

Vortex Genie 2 ${ }^{\mathrm{TM}}$ (Bender \& Hobein, Zürich, Schweiz)

\section{Additional equipment}

Single use articles were purchased from the following companies: Eppendorf (Hamburg), Falcon (Heidelberg), Schütt (Göttingen), Greiner (Frickenhausen), Heinemann (Duderstadt), Qiagen (Hilden), Sarstedt (Langenhagen).

\subsubsection{Software}

Windows XP, Microsoft Word, Microsoft Excel, Microsoft PowerPoint, DNA Star, ImageQuant, Freehand, Adobe Photoshop, EndNote, Unicorn, Boxshade, Spot

\subsection{Methods}

\subsubsection{Molecular biological methods}

\subsubsection{Culturing prokaryotic cells}

E. coli cells were used for the propagation of plasmid DNA or the expression of recombinant proteins.

\subsection{Bacterial cell maintenance}

The bacteria were streaked onto the surface of an LB agar plate or were directly added to liquid media containing appropriate antibiotics. The agar plates and liquid cultures were incubated at $37^{\circ} \mathrm{C}$ for $12-16$ hours. A single culture of the agar plate was used to inoculate 3-5 ml liquid medium.

Bacteria carrying plasmid DNA were selected by adding antibiotics to the cultures. The plasmid DNA contains a gene for a certain antibiotic resistance that enables the bacteria to metabolize the antibiotic while cells without the corresponding plasmid DNA die. 


\subsection{Storage of bacteria}

Bacteria can be stored for several years in cultures containing glycerol. For long term storage, $0.5 \mathrm{ml}$ of the respective culture were mixed with $0.5 \mathrm{ml}$ glycerol and frozen at $-80^{\circ} \mathrm{C}$. Working cultures were stored at $-20^{\circ} \mathrm{C}$. To recover bacteria, cultures were used to inoculate liquid media or streaked onto a LB agar plate containing suitable antibiotics.

\subsubsection{Preparation of competent bacterial cells}

Bacterial cells are called "competent" when they are able to take up foreign (vector) DNA. A calcium chloride treatment was performed for preparation of competent E. coli cells. This method is based on the permeability of the bacterial cell membrane to chloride ions while it is non-permeable to calcium ions. Water molecules enter the bacterial cells together with the chloride ions thereby causing the cells to swell which is necessary for subsequent DNA uptake.

A glycerol stock of the respective E. coli strain was used to inoculate $10 \mathrm{ml}$ LB medium without antibiotics growing at $37^{\circ} \mathrm{C}$ overnight. $2-3 \mathrm{ml}$ of this pre-culture were added to $100 \mathrm{ml} \mathrm{LB}$ and the culture was grown at $37^{\circ} \mathrm{C}$ to an optical density of 0.6 at $600 \mathrm{~nm}$. The cells were cooled down on ice for at least $30 \mathrm{~min}$, followed by centrifugation for $10 \mathrm{~min}$ at $4000 \mathrm{rpm}$ and $4^{\circ} \mathrm{C}$ and were finally resuspended in $20 \mathrm{ml}$ ice-cold sterile calcium chloride $(50 \mathrm{mM})$. After further incubation on ice (at least $30 \mathrm{~min}$ ) the cells were centrifuged again, resuspended in $4.5 \mathrm{ml}$ calcium chloride solution and incubated on ice for $30 \mathrm{~min}$. Glycerol was added to a final concentration of $10 \%$ and $120 \mu \mathrm{l}$ aliquots of the competent cells were frozen in liquid nitrogen and stored at $-80^{\circ} \mathrm{C}$.

\subsubsection{Bacterial cell transformation}

The mechanism by which bacterial cells take up plasmid DNA is not well understood. However, the calcium chloride treatment during the preparation of competent cells and a heat-shock after addition of the plasmid DNA facilitate the uptake.

An aliquot of competent cells was thawed on ice and 50-100 ng of plasmid DNA or a DNA ligation reaction was added subsequently. After $30 \mathrm{~min}$ of incubation on ice the mixture was heat-shocked in a water bath at $42^{\circ} \mathrm{C}$ for $90 \mathrm{sec}$. The cells were again incubated on ice for $5 \mathrm{~min}$ and then $300 \mu \mathrm{l}$ of antibiotic-free medium were added. The bacteria were incubated for $1 \mathrm{~h}$ at $37^{\circ} \mathrm{C}$ to regenerate and to allow expression of the antibiotic resistance gene. Finally, the cells were plated onto a LB agar plate containing the appropriate antibiotic and were incubated at $37^{\circ} \mathrm{C}$ overnight. 


\subsubsection{Plasmid purification}

\subsection{TELT method}

The isolation of analytical amounts of plasmid DNA from single colonies with a buffer containing Tris, EDTA, $\mathrm{LiCl}$ and Triton X-100 (TELT) followed by restriction enzyme digestion analysis (chapter 4.2.1.7) was performed to screen for "positive" colonies after cloning and transformation of plasmids. For that, $3 \mathrm{ml} \mathrm{LB}$ medium containing the appropriate antibiotics were inoculated with individual clones from the agar plate and were incubated at $37^{\circ} \mathrm{C}$ overnight. $1.5 \mathrm{ml}$ of each culture were centrifuged for $5 \mathrm{~min}$ at $5000 \mathrm{rpm}$ and the collected cells were resuspended in $150 \mu \mathrm{l}$ TELT buffer [50 mM Tris-HCl, $\mathrm{pH}$ 7.5; $62.5 \mathrm{mM}$ EDTA, $2.5 \mathrm{M} \mathrm{LiCl,}$ $0.4 \%(\mathrm{w} / \mathrm{v})$ Triton $\mathrm{X}-100] .15 \mu 1$ lysozyme $\left(10 \mathrm{mg} / \mathrm{ml}\right.$ in $\left.\mathrm{dH}_{2} \mathrm{O}\right)$ were added and the solution was boiled for $2 \mathrm{~min}$ at $95^{\circ} \mathrm{C}$ and subsequently cooled on ice for $5 \mathrm{~min}$. After centrifugation for $10 \mathrm{~min}$ at $14000 \mathrm{rpm}$ the pellet containing cell debris and denatured proteins was removed with a toothpick. The plasmid DNA was precipitated with $150 \mu 1$ isopropanol and centrifugation for $10 \mathrm{~min}$ at $14000 \mathrm{rpm}$. The DNA-pellet was washed with $100 \mu 170 \%$ ethanol, dried in a Speed

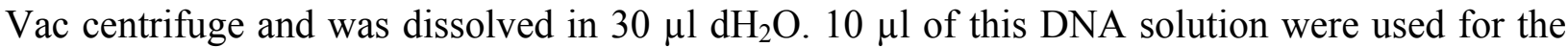
analytical restriction enzyme digestion.

\subsection{Preparative plasmid purification}

Plasmid DNA that was used for e.g. preparative restriction enzyme digestion, DNA sequencing, and transfection of eukaryotic cells was purified with the following kits depending on the desired amount of purified DNA: E.Z.N.A. Plasmid-Miniprep Kit II (Peqlab, Erlangen), NucleoSpin ${ }^{\circledR}$ Plasmid (Macherey-Nagel, Düren), and Qiagen Plasmid-Maxi/Midi-Kit (Qiagen, Hilden). The plasmid purification was performed according to the manufacturer's instructions. In all cases, the purification was based on a modified alkaline lysis, followed by binding of plasmid DNA to anion-exchange columns thereby removing RNA, proteins and other low-molecular-weight impurities.

\subsubsection{Concentration of DNA by ethanol/isopropanol precipitation}

For DNA precipitation either ethanol or isopropanol was used. 2-3 volumes of ethanol were added to the DNA solution and the mixture was incubated at $-80^{\circ} \mathrm{C}$ for $1 \mathrm{~h}$. After centrifugation the DNA was washed with $70 \%$ ethanol, centrifuged again and dried either on air or in a Speed Vac centrifuge. (DNA precipitation with ethanol can also be performed in the presence of sodium acetate. In this case, incubation at $-80^{\circ}$ is not necessary.) 
DNA precipitation was also performed adding 0.7 volumes of isopropanol. The mixture was centrifuged and the DNA was washed with $70 \%$ ethanol and subsequently dried.

\subsubsection{Quantitation of DNA}

The concentration and purity of DNA was determined by spectrophotometric measurements at $260 \mathrm{~nm}$ and $280 \mathrm{~nm}$. An optical density of 1.0 at $260 \mathrm{~nm}$ corresponds to approximately $50 \mu \mathrm{g} / \mathrm{ml}$ double-stranded (ds) DNA and $40 \mu \mathrm{g} / \mathrm{ml}$ single-stranded (ss) DNA or RNA. Aromatic amino acid residues and phenol absorb at $280 \mathrm{~nm}$. Therefore, the ratio between the readings at $260 \mathrm{~nm}$ and $280 \mathrm{~nm}$ indicates the purity of DNA. Pure DNA without protein contamination has a $\mathrm{OD}_{260} / \mathrm{OD}_{280}$ ratio of $1.8-2$.

To only estimate the amount of plasmid DNA an aliquot of the DNA plus a size marker of known concentrations were analyzed by agarose gel electrophoresis (see chapter 4.2.1.11). The fluorescence intensities of the intercalated ethidium bromide were compared and the DNA concentration was assessed.

\subsubsection{Restriction enzyme digestion}

Restriction enzymes bind to double-stranded DNA within a specific recognition sequence that is in most cases palindromic. Depending on the enzyme the DNA is cleaved at the axis of the twofold symmetry generating blunt ends or at opposite sites of the axis of symmetry leading to sticky ends.

The DNA was incubated with the enzyme and the respective buffer at the optimal temperature according to the manufacturer's instructions. For analytical digestion $0.5-2 \mu \mathrm{g}$ DNA or for preparative digestion 5-10 $\mu$ g DNA were incubated with 1-5 U of enzyme per $\mu$ g of DNA for 2-3 h. Analytical digestion was used to screen for 'positive' clones after transformation and plasmid preparation with the TELT method (see chapter 4.2.1.4.1). When digestion products smaller than $200 \mathrm{bp}$ were expected the bacterial RNA had to be additionally digested with $0.5 \mu 1$ RNaseA (10 mg/ml) to identify 'positive' clones after agarose gel electrophoresis.

\subsubsection{Dephoshorylation of digested plasmid vectors}

To prevent self-ligation of linearized vectors the $5^{\prime}$-phospate was removed with calf intestinal alkaline phosphatase (CIP); especially important when only one restriction enzyme was used. Alkaline phosphatase catalyzes the removal of 5'-phosphate residues from DNA and other substrates such as RNA. 
After restriction enzyme digestion, the vector DNA was incubated with $10 \mathrm{U}$ CIP and 1/10 CIP buffer for $30-60 \mathrm{~min}$ at $37^{\circ} \mathrm{C}$. If necessary, CIP was heat-inactivated at $75^{\circ} \mathrm{C}$ for $10 \mathrm{~min}$ in the presence of $5 \mathrm{mM}$ EDTA. However, in most cases ligations were performed without inactivating CIP.

\subsubsection{Polymerase chain reaction (PCR)}

The PCR was used to amplify DNA fragments using two oligonucleotide primers complementary to the sequences flanking the coding region of interest. Additionally, these primers contain recognition sequences for restriction enzymes at their 5'-end. Long DNA fragments were amplified with the polymerase $\operatorname{Vent}_{R}{ }^{\circledR}$ (New England BioLabs) because of its proof-reading exonuclease activity. Shorter fragments or fragments that should contain adenine protrusions for cloning into the pGEM ${ }^{\circledR}-\mathrm{T}-$ Easy vector were amplified with RedTaq ${ }^{\mathrm{TM}}$ polymerase (Sigma). A typical reaction contains $100 \mathrm{ng}$ of template DNA, 100 pmol of forward and reverse primer, $5 \mu \mathrm{l}$ of $2 \mathrm{mM}$ dNTPs, 1/100 DMSO (v/v), 1/10 (v/v) of the polymerase buffer (10x) and $2 \mathrm{U}$ Vent or $3 \mathrm{U}$ RedTaq polymerase in a total volume of $100 \mu$. The PCR was performed in a thermocycler (Biometra, Perkin Elmer) using the following program: 35 cycles of strand denaturation for $1 \mathrm{~min}$ at $95^{\circ} \mathrm{C}$, primer annealing for $1 \mathrm{~min}$, and primer extension (elongation) at $72^{\circ} \mathrm{C}$, followed by a final elongation step for $7 \mathrm{~min}$ at $72^{\circ} \mathrm{C}$. The annealing temperature $\left(\mathrm{T}_{\mathrm{A}}\right)$ depends on the melting temperature $\left(\mathrm{T}_{\mathrm{m}}\right)$ of both primers and can be calculated by the following formula: $T_{A}=\left(T_{m} 1+T_{m} 2\right) / 2-3^{\circ} \mathrm{C}$. The elongation time depends on the length of the DNA fragment and the velocity of the polymerase (usually 1 min per $1000 \mathrm{bp}$ ). An aliquot of the amplified fragments was analyzed by agarose gel electrophoresis. After purification with the E.Z.N.A Cycle-Pure Kit (Peqlab) according to the manufacturer's instructions the DNA fragments were digested with the appropriate restriction enzymes (see chapter 4.2.1.7).

\subsubsection{Site directed mutagenesis}

Site directed mutagenesis was performed using two different methods. Amino acids at the very C-terminus of a protein (approximately the last 20 amino acids) can be easily mutated using an antisense amplification primer carrying the desired mutation. Other amino acids were mutated by site-directed mutagenesis according to the Quick Change Site-directed Mutagenesis Kit protocol (Stratagene).

Nucleotide exchanges in DFF40mutA (pSN93, pSN99), DFF40mutB (pSN92, pSN98) and DFF45mutB (pSN94, pSN100) were inserted using the antisense amplification primers: 5'AATAGTCGACTCACTGGCGTTTCCGCACAGGCTGCGCTGCAGCCAAAGCTGTCTGGG 
GTT-3' for DFF40mutA, 5'-AATAGTCGACTCACTGGGCTGCCGCCACAGGCTGCTTCCG -3' for DFF40mutB, and 5'-AATAGTCGACCTATGTGGGATCCTGTGCGGCTGCCGCAGG ATTCTGCAGG-3’ for DFF45mutB.

To generate DFF45mutA (pSN97, pSN101), DFF40mutC (pSN107, pSN108, pSN109) and importin $\alpha \mathrm{E} 388 \mathrm{R}$ (pSN103) site-directed mutagenesis was performed according to the Quick Change Site-directed Mutagenesis Kit protocol (Stratagene). Two complementary oligonucleotides containing the desired mutation were used to introduce site-specific mutations in double stranded plasmid DNA. The reaction mixture contained $500 \mathrm{ng}$ template DNA, 13 pmol of both complementary oligonucleotides, $5 \mu \mathrm{l}$ of $2 \mathrm{mM}$ dNTPs, $2 \mathrm{mM} \mathrm{MgSO}_{4}$, $1 / 10$ polymerase buffer $(10 \mathrm{x})$ and $2 \mathrm{U}$ Vent ${ }^{\circledR}$ polymerase in a total volume of $50 \mu 1.25 \mu 1$ of this mixture were incubated at room temperature as negative control. The PCR was performed with the remaining $25 \mu \mathrm{l}$ using the following program: 28 cycles of strand denaturation for $30 \mathrm{sec}$ at $95^{\circ} \mathrm{C}$, primer annealing for $1 \mathrm{~min}$ at $65^{\circ} \mathrm{C}$, and elongation at $72^{\circ} \mathrm{C}$, followed by a final elongation step for $7 \mathrm{~min}$ at $72^{\circ} \mathrm{C}$. The annealing temperature depends on the melting temperature $\left(\mathrm{T}_{\mathrm{m}}\right)$ of the primer for which the mismatch of exchanged nucleotides has to be considered. The corrected melting temperature can be calculated by the following formula: $\mathrm{T}_{\mathrm{m}}=81.5+0.41 \cdot(\% \mathrm{GC})-675 / \mathrm{N}-$ $\%$ mismatch ( $\mathrm{N}$ is the number of bases of the oligonucleotide). However, the annealing step was generally carried out at $65^{\circ} \mathrm{C}$. The elongation time depends on the length of the vector and the velocity of the polymerase ( $1 \mathrm{~min}$ per $1000 \mathrm{bp}$ for Vent ${ }^{\circledR}$ polymerase). After the PCR, $0.5 \mu 1$ $D p n I$ restriction enzyme $(10 \mathrm{U} / \mu \mathrm{l})$ were added to the reaction mixture and to the negative control. Both were incubated for $1.5-3 \mathrm{~h}$ at $37^{\circ} \mathrm{C}$. DpnI specifically cleaves methylated and hemimethylated DNA. Therefore, the methylated template DNA that was isolated from E. coli is digested while the amplified unmethylated vector carrying the mutation remains intact. $5 \mu 1$ of each, the mutation reaction and the negative control, were used to transform E. coli DH5 $\alpha$ cells. The following complementary oligonucleotides were used for site-directed mutagenesis of DFF45mutA, DFF40mutC and importin $\alpha$ E388R: 5'-AGCTTGCATTCTCTCGCGAGCATCTC AGCAAGCGCGGCCTCACCACCTGG-3' (sense) and 5'-TCGAACGTAAGAGAGCGCTCG TAGAGTCGTTCGCGCCGGAGTGGTGGACC-3' (antisense) for DFF45mutA, 5'-TAAAACT AGTGCACATTGTCTGCCATGCGGCAACCACCCACAAGCTC-3' (sense) and 5'-GAGCTT GTGGGTGGTTGCCGCATGGCAGACAATGTGCACTAGTTTTA-3' (antisense) for DFF40 mutC, and 5'-TTCAAGGCCCAGAAACGAGCAGTTTGGGCTGTA-3' (sense) and 5'-AAGT TCCGGGTCTTTGCTCGTCAAACCCGACAT-3’ (antisense) for importin $\alpha E 388 \mathrm{R}$. 


\subsubsection{Agarose gel electrophoresis}

Agarose gel electrophoresis was performed to analyze or to purify DNA fragments, e.g. after PCR reactions and restriction enzyme digestions.

Depending on the fragment size $0.8-2 \%(\mathrm{w} / \mathrm{v})$ agarose in TBE buffer [90 $\mathrm{mM}$ Tris base $(\mathrm{pH} 8.3)$, $90 \mathrm{mM}$ boric acid, $2.5 \mathrm{mM}$ EDTA] was used to prepare the gels. Prior to loading, the DNA solution was mixed with $1 / 5(\mathrm{v} / \mathrm{v})$ gel loading buffer [40 \% (w/v) sucrose in water, $0.1 \%(\mathrm{w} / \mathrm{v})$ bromophenol blue, 0.1 M EDTA]. To determine the size of the DNA fragments appropriate size markers were used. The electrophoresis was carried out at 90-110 V at room temperature. The DNA was stained with the fluorescent intercalating dye ethidium bromide (Sharp et al., 1973) by incubating the gels for 15 minutes in an ethidium bromide solution ( $3 \mu \mathrm{g}$ ethidium bromide in 11 water). The DNA was visualized under UV light (radiation at $302 \mathrm{~nm}$ ) or when the fragments should be recovered and subsequently purified under UV light with longer wave length (radiation at $366 \mathrm{~nm}$ ) to avoid DNA damage.

\subsubsection{Elution of DNA from agarose gels}

Purification of DNA fragments from agarose gels was carried out using the QIAEX ${ }^{\circledR}$ II Agarose Gel Extraction Kit (Qiagen) according to the manufacturer's instructions. Briefly, after excision of the band of interest with a scalpel, three times the volume QX1 solution was added and the gel was melted at $50^{\circ} \mathrm{C}$. Subsequently, $15 \mu \mathrm{l}$ of glass beads were added to the solution for DNA binding and the mixture was further incubated for $10 \mathrm{~min}$ at $50^{\circ} \mathrm{C}$. The solution was centrifuged for $1 \mathrm{~min}$ at $13000 \mathrm{rpm}$ and the beads were washed three times, once with QX1 and twice with PE buffer. The beads were air-dried and the DNA was eluted with $30 \mu \mathrm{l}$ Tris-HCl buffer (pH 8.5) or $\mathrm{dH}_{2} \mathrm{O}$.

\subsubsection{DNA ligation}

For the ligation of linearized cloning vector and insert DNA, both cleaved with the same restriction enzymes, T4 DNA ligase was used which catalyzes the formation of phosphodiester bonds between adjacent 3'-hydroxyl and 5'-phosphate termini. Ligations were performed by incubation of about $100 \mathrm{ng}$ of linearized vector with a five times molar excess of insert DNA in the presence of T4 ligase buffer and 0.5 U T4 DNA ligase (New England Biolabs). The reactions were incubated for $2-3 \mathrm{~h}$ at $25{ }^{\circ} \mathrm{C}$ or overnight at $4{ }^{\circ} \mathrm{C}$. In addition, control ligations were performed in the absence of insert DNA to check for self-ligation of inefficiently cleaved or dephosphorylated vector. 


\subsubsection{Annealing of single stranded oligonucleotides}

Complementary single stranded oligonucleotides were used to integrate very small DNA fragments coding for e.g. particular protein regions of interest (like NLSs), affinity tags or enzyme cleavage sites into cleaved vector DNA. For that, the oligonucleotides have to be annealed because only double stranded DNA is integrated. $5 \mu \mathrm{l}(500 \mathrm{pmol})$ of both oligonucleotides were mixed with $40 \mu \mathrm{dH}_{2} \mathrm{O}$ and heated in a large water bath (about 11 ) for 5 min at $95^{\circ} \mathrm{C}$. The water bath was then allowed to slowly cool down to room temperature. The oligonucleotides contain overhanging restriction digestion sites at both ends, therefore they can be directly integrated into cleaved vector DNA containing the 5'-phosphate. In case of dephosphorylated vector DNA (see chapter 4.2.1.8), especially when only one restriction enzyme was used, the annealed oligonucleotides had to be phosphorylated (see below).

\subsubsection{End-labeling of DNA with T4 polynucleotide kinase (T4 PNK)}

T4 PNK was used to phosphorylate double-stranded oligonucleotides that should be ligated with dephosphorylated vector DNA. T4 PNK catalyzes the transfer of the $\gamma$-phosphate of ATP to a 5'-hydroxyl terminus of DNA or RNA. A reaction mixture contained 50 pmol double stranded oligonucleotide ( $5 \mu \mathrm{l}$ of the reaction described in chapter 4.2.1.14), $1 \mathrm{mM} \mathrm{ATP,} 1 / 10$ PNK buffer (10x buffer: $0.07 \mathrm{M}$ Tris/HCl pH 7.6) and $10 \mathrm{U}$ T4 PNK (10 U/ $\mu$ l) in a total volume of $20 \mu 1$.

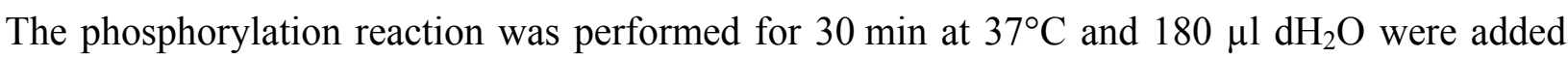
subsequently. Usually, $1 \mu \mathrm{l}$ of this solution was used for the ligation reaction.

\subsubsection{DNA sequencing}

The nonradioactive sequencing of double stranded DNA is based on the Sanger chain termination method (Sanger et al., 1977). The sequencing reactions on DNA templates of interest were performed using one specific primer and the Dye Terminator Ready Mix (Big Dye ${ }^{\mathrm{TM}}$ Terminator Sequencing Kit, Applied Biosystems) containing the DNA polymerase, 2'-deoxynucleotide 5'-triphosphates (dNTPs) and 2',3'-dideoxynucleotide 5'-triphospates (ddNTPs). ddNTPs lack the 3'-hydroxyl group and are therefore not able to form a phosphodiester bond with the succeeding dNTP which leads to the termination of the chain reaction. Hence, the generated DNA fragments are of different lengths depending on when a ddNTP was integrated into the growing strand and can be separated by size in a capillary electrophoresis. The four ddNTPs are tagged with four different fluorescent dyes which allows the detection of the sequencing products by laser scanning in an $\mathrm{ABI}$ sequencer (model 373A, Applied Biosystems). 
A sequence reaction contained $400 \mathrm{ng}$ DNA, 100 pmol primer, 1,5 $\mu 1$ sequence buffer and 1,5 $\mu 1$ Dye Terminator Ready Mix in a total volume of $10 \mu 1$. The PCR protocol includes 25 cycles of strand denaturation for $30 \mathrm{sec}$ at $95^{\circ} \mathrm{C}$, primer annealing for $15 \mathrm{sec}$ at $50^{\circ} \mathrm{C}$ and chain elongation for $4 \mathrm{~min}$ at $60^{\circ} \mathrm{C}$. Then $1 \mu 13 \mathrm{M}$ sodium acetate, $1 \mu 1125 \mathrm{mM}$ EDTA, and $50 \mu 1$ ethanol were added and the mixture was centrifuged for $15 \mathrm{~min}$ at $14000 \mathrm{rpm}$ and $4^{\circ} \mathrm{C}$ to precipitate the DNA. After washing with $70 \mu 1$ of $70 \%$ ethanol, the DNA was dried in a Speed Vac centrifuge for 2 min and was dissolved in $30 \mu 1 \mathrm{H}_{2} \mathrm{O}$ (HPLC quality). The subsequent capillary electrophoresis analysis was done by Andreas Nolte in the Department of Developmental Biochemistry (University of Göttingen).

\subsubsection{Expression constructs}

The coding regions of the respective genes were amplified from plasmid DNA using specific primer pairs with appropriate restriction sites. All constructs were verified by DNA sequencing (for a list of expression constructs used in this study see Appendix, Tab. I).

\subsection{Bacterial expression constructs}

The coding regions for the GST-fusion proteins were cloned as follows: human full length DFF40 (pSN8) and DFF40 $324-338$ (pSN30) as XbaI/HindIII fragments into the SpeI/HindIII sites of pET-41a(+) (Novagen); DFF40(aa314-338) (pSN 60), human full length DFF45 (pSN27), DFF45_306-331 (pSN52) and DFF45(aa296-331) (pSN61) as EcoRI/XhoI fragments into the respective sites of pGEX-4T-1 (Amersham).

The coding regions for the His-tagged proteins were cloned as follows: human full length DFF40 (pSN72) and DFF40 $4324-338$ (pSN73) as NdeI/HindIII fragments into the respective sites of pET-28b(+) (Novagen); DFF45(aa8-331) (pWG3; kindly provided by Wiebke Göbel) as $K p n I / H i n d I I I$ fragment into the respective sites of pQE30 (Qiagen); DFF45 306-331 (pSN74) as EcoRI/HindIII fragment into the respective sites of pETDuet-1 (Novagen).

The expression construct for the EGFP-DFF40 (pSN33) fusion protein was cloned as follows: human DFF40 as SalI/HindIII fragment into the respective sites of pET-41-EGFP (pSN 31). pET-41-EGFP is a modified pET-41a(+) vector in which GST was replaced by EGFP, cloning the corresponding coding region into the $N d e \mathrm{I} / N c o$ I sites.

\subsection{Eukaryotic expression constructs}

The EGFP-GST-fusion proteins were cloned as follows: the coding regions or gene fragments of human DFF40 (pSN77, pSN79) and DFF45 (pSN81, pSN83) as HindIII/SalI fragments into 
the respective sites of pEGFP-GST-C1 [pSN55; modified pEGFP-C1 (Clontech) in which GST was inserted N-terminally of the MCS as BglII/XhoI fragment].

The RFP-fusion proteins were cloned as follows: the coding regions or gene fragments of human DFF40 (pSN86, pSN87) and DFF45 (pSN88, pSN89) as HindIII/SalI fragments into the respective sites of pRFP-C1 (modified pEGFP-C1 in which EGFP was replaced by RFP using the restriction sites $N h e \mathrm{I} / B g / \mathrm{II})$.

The coding regions for the EGFP-EGFP-GST-fusion proteins were cloned as follows: gene fragments of DFF40 (pSN106) and DFF45 (pSN69) as HindIII/EcoRI fragments into the respective sites of pEGFP-EGFP-GST-C1 (modified pEGFP-GST-C1 vector in which a second EGFP was inserted at the N-terminus as NheI fragment). The pEGFP-EGFP-GST-C1 vector was a kind gift of Saskia Hutten and Ralf Kehlenbach (Institute for Biochemistry and Molecular Cellbiology, Department Biochemistry I, University of Göttingen).

\subsubsection{Biochemical methods}

\subsubsection{SDS-polyacrylamide gel electrophoresis (SDS-PAGE)}

Sodium dodecyl sulfate (SDS) is a strongly anionic detergent that wraps its hydrophobic tail around the polypeptide backbone of proteins, thereby denaturing them and conferring a net negative charge. For almost all proteins, the amount of SDS binding to the protein is proportional to its molecular weight. Therefore, the migration of proteins in SDS-PAGE separations is determined by their molecular weight instead of their intrinsic electric charge (Weber and Osborn, 1969). However, additional reducing agents like $\beta$-mercaptoethanol (BME) or dithiotreitol (DTT) must be used to completely unfold the proteins.

SDS-PAGE was performed using a discontinuous buffer system with a stacking and a resolving gel (Laemmli, 1970). Thereby, the buffers used to prepare the gel and the reservoir buffer have different $\mathrm{pH}$ values and ionic strengths. Before proteins are separated according to their size in the resolving gel they are concentrated in the stacking gel which greatly increases the resolution of the SDS-PAGE. The gels are composed of polyacrylamide chains crosslinked by N,N'methylenebisacrylamide (bisacrylamide). The polymerization reaction occurs in the presence of free radicals supplied by ammonium persulfate and is catalyzed by TEMED. The sieving properties of the gel are determined by the size of the pores which depends on the concentration of acrylamide/bisacrylamide. Concentrations from $5 \%$ to $15 \%$ allow separations of proteins in a range from $250 \mathrm{kDa}$ to $12 \mathrm{kDa}$.

SDS-PAGE was carried out in vertical gels using mini-gel chambers (Biometra). Buffers and gels (Tab. 2) were composed as follows: $3 \times$ sample buffer: $9 \%$ (w/v) SDS, $300 \mathrm{mM}$ Tris-HCl 
$\mathrm{pH}$ 6.7, $22.5 \%(\mathrm{w} / \mathrm{v})$ glycerol, $0.1 \%(\mathrm{w} / \mathrm{v})$ bromophenol blue, $10 \%(\mathrm{v} / \mathrm{v}) \beta$-mercaptoethanol (BME); stacking gel buffer: $0.5 \mathrm{M}$ Tris-HCl pH 6.8, $0.4 \%(\mathrm{w} / \mathrm{v}) \mathrm{SDS}$; resolving gel buffer: 1.5 M Tris-HCl pH 8.8, 0.4 \% (w/v) SDS; and 5x 'Laemmli' buffer: $0.25 \mathrm{M}$ Tris, $10 \mathrm{mM}$ EDTA, 1.9 M glycine, $\mathrm{pH} 8.7,0.5 \%$ (w/v) SDS.

Table 2: Composition of SDS-polyacrylamide gels (sufficient for 5-6 minigels)

\begin{tabular}{|l|c|c|c|c|}
\hline & \multicolumn{3}{|c|}{ resolving gel } & stacking gel \\
\hline & $10 \%$ & $12.5 \%$ & $15 \%$ & \\
\hline $\mathrm{dH}_{2} \mathrm{O}$ & $12.4 \mathrm{ml}$ & $9.9 \mathrm{ml}$ & $7.4 \mathrm{ml}$ & $6.2 \mathrm{ml}$ \\
\hline resolving gel buffer & $7.5 \mathrm{ml}$ & $7.5 \mathrm{ml}$ & $7.5 \mathrm{ml}$ & - \\
\hline stacking gel buffer & - & - & - & $2.5 \mathrm{ml}$ \\
\hline acrylamide/bisacrylamide $(30: 0.8)$ & $10 \mathrm{ml}$ & $12.5 \mathrm{ml}$ & $15 \mathrm{ml}$ & $1.3 \mathrm{ml}$ \\
\hline TEMED & $0.02 \mathrm{ml}$ & $0.02 \mathrm{ml}$ & $0.02 \mathrm{ml}$ & $0.02 \mathrm{ml}$ \\
\hline $10 \%$ APS & $0.4 \mathrm{ml}$ & $0.4 \mathrm{ml}$ & $0.4 \mathrm{ml}$ & $0.4 \mathrm{ml}$ \\
\hline
\end{tabular}

Before loading on the stacking gel the protein samples were heated for 5 min at $95^{\circ} \mathrm{C}$ in $3 \mathrm{x}$ sample buffer. Molecular weight markers were used to estimate the molecular weight of the polypeptide chains. For migration through the stacking gel an electric current of 15-20 mA was applied and was then increased to $20-25 \mathrm{~mA}$ once the proteins had entered the resolving gel. After electrophoresis proteins were detected in the gel or transferred onto a nitrocellulose membrane by western blotting (chapter 4.2.2.2.1).

For detection of non-radioactive proteins, the gels were fixed and stained with a methanol/glacial acetic acid solution containing Coomassie Brilliant Blue (10\% glacial acetic acid, 50\% methanol, $2.5 \mathrm{~g} / 1$ Coomassie Brilliant Blue R250). Subsequently, the gels were first destained in a 'strong' destaining solution (50\% methanol, 10\% acetic acid) and then in a 'weak' destaining solution (10\% methanol, $5 \%$ acetic acid).

SDS-polyacrylamide gels containing radioactively labeled amino acids ( ${ }^{35} \mathrm{~S}$-labeled) had to be dried before autoradiography. The gel was covered with a foil and sandwiched between Whatman paper to minimize shrinkage and distortion and was dehydrated on the vacuum gel dryer (BioRad) at $70^{\circ} \mathrm{C}$. Prior to phosphorimaging (GE Healthcare) the gel was exposed on a phosphorimager screen for 1 to 3 days. 


\subsubsection{Immunoblotting}

\subsection{Semidry-Western blot}

To transfer proteins separated by SDS-PAGE from the gel onto a nitrocellulose membrane (Whatman, Dassel) the so-called semidry-Western blot was performed. Thereby, the gel was placed onto the nitrocellulose membrane and sandwiched between Whatman paper. Before setting up the blotting device, gel, nitrocellulose membrane and Whatman papers, all of the same size, were soaked in blotting buffer [25 mM Tris, $150 \mathrm{mM}$ glycine and $10 \%(\mathrm{v} / \mathrm{v})$ methanol]. The protein transfer was carried out between graphite electrodes (rinsed with $\mathrm{dH}_{2} \mathrm{O}$ before) at room temperature for 1.5-2 $\mathrm{h}$ applying an electric current of $1 \mathrm{~mA} / \mathrm{cm}^{2}$. Afterwards the proteins were stained with Ponceau S solution (AppliChem, Darmstadt) to visualize the transferred proteins.

\subsection{Detection of immobilized proteins using antibodies}

Proteins immobilized on nitrocellulose membranes can be detected using a specific primary antibody and an enzyme-conjugated secondary antibody. The secondary antibodies used in the present work were conjugated to horseradish peroxidase (HRP). HRP together with hydrogen peroxide catalyzes the oxidation of luminol in alkaline conditions leading to the emission of light (chemiluminescence) which can be detected with chemiluminescence films.

To avoid unspecific binding of the antibodies to the nitrocellulose the membrane was incubated with a blocking solution [(5\% milk powder in TBST $(20 \mathrm{mM}$ Tris- $\mathrm{HCl} \mathrm{pH} 7.6,14 \mathrm{mM} \mathrm{NaCl}$, $0.01 \%$ Tween 20)] for $1-4 \mathrm{~h}$ at room temperature or at $4{ }^{\circ} \mathrm{C}$ overnight. Afterwards, the incubation with the primary antibody was carried out for $2 \mathrm{~h}$ at room temperature or overnight at $4^{\circ} \mathrm{C}$. In general, primary antibodies were diluted in blocking solution. However, some antibody solutions can be reused for subsequent immune reactions. In this case dilution in TBS (20 mM Tris-HCl $\mathrm{pH} 7.6,14 \mathrm{mM} \mathrm{NaCl}$ ) containing $5 \% \mathrm{BSA}$ and $0.02 \%$ sodium azide might be more suitable (appropriate conditions have to be determined for each antibody). Antibodies in TBS were stored at $4{ }^{\circ} \mathrm{C}$ while antibodies in blocking solution were frozen at $-20^{\circ} \mathrm{C}$.

After incubation with the primary antibody, the nitrocellulose membrane was washed three times with TBST. The secondary antibody diluted in blocking solution was applied at room temperature for $1 \mathrm{~h}$ in case of a $2 \mathrm{~h}$ incubation with the primary antibody or for $2 \mathrm{~h}$ when the primary antibody was applied overnight. Afterwards, the nitrocellulose membrane was washed four times (2x $5 \mathrm{~min}, 2 \mathrm{x} 10 \mathrm{~min}$ ) with TBST and bound antibodies were detected using the ECL $^{\mathrm{TM}}$ Western Blotting Detection Reagents and Hyperfilm ${ }^{\mathrm{TM}}$ ECL (GE Healthcare) according to the manufacturer's instructions. 
To remove bound antibodies, the nitrocellulose membrane was incubated for $15 \mathrm{~min}$ at $65^{\circ} \mathrm{C}$ in stripping buffer [62,5 mM Tris- $\mathrm{HCl} \mathrm{pH}$ 6.7, 2\% SDS (w/v), $100 \mathrm{mM} \mathrm{BME]} \mathrm{and} \mathrm{was}$ subsequently washed in TBST. After incubation in blocking solution, the membrane was incubated with another primary antibody and the respective secondary antibody.

\subsubsection{Bradford protein assay}

The Bradford assay was used to determine the total protein concentration of a sample (Bradford, 1976). This assay is based on the color shift of Coomassie Brilliant Blue G250 after binding to proteins. Bound proteins stabilize the anionic form of the dye through ionic and hydrophobic interactions. Thereby, the absorbance maximum of Coomassie Brilliant Blue G250 is shifted from $465 \mathrm{~nm}$ to $595 \mathrm{~nm}$. The amount of protein in the solution is proportional to the increase in absorbance. BSA in the range of 0 to $20 \mu \mathrm{g} / \mathrm{ml}$ was used as a protein standard. The different BSA solutions and the protein samples were diluted in the same buffer (total volume $50 \mu 1$ ) and $1 \mathrm{ml}$ Bradford reagent $\left(425 \mathrm{ml} \mathrm{dH_{2 }} 0,15 \mathrm{ml} \mathrm{95 \%}\right.$ ethanol, $30 \mathrm{ml} \mathrm{88 \%} \mathrm{phosphoric} \mathrm{acid,} \mathrm{and} 30 \mathrm{ml}$ Bradford stock solution) was added. After 10 min incubation, the absorbance was measured at $595 \mathrm{~nm}$. The Bradford stock solution consisted of $100 \mathrm{ml} \mathrm{95 \%} \mathrm{ethanol,} 200 \mathrm{ml} \mathrm{88 \%} \mathrm{phosphoric}$ acid, and 350 mg Coomassie Brilliant Blue G-250.

\subsubsection{Recombinant protein expression and purification}

Recombinant proteins were expressed in appropriate $E$. coli strains. In general, a $50 \mathrm{ml}$ culture of the selected clone was grown at $37^{\circ} \mathrm{C}$ overnight. Next day the culture was scaled up to 11 and was further grown at $37^{\circ} \mathrm{C}$ to an optical density of $0.9-1.0$ at $600 \mathrm{~nm}$. Protein expression was induced with isopropylthio-ß-D-galactoside (IPTG) which inactivates the bacterial lac repressor protein thereby inducing transcription of the gene. The concentration of IPTG and the expression temperature and time depended on the protein. After expression, the culture was cooled on ice and the cells were collected by centrifugation for $10 \mathrm{~min}$ at $6000 \mathrm{rpm}$ (GSA rotor, Sorvall®). After resuspending the cells in a suitable buffer, they were frozen in liquid nitrogen, thawed and lyzed by sonication for $3 \mathrm{x} 40 \mathrm{sec}$ (with a $10 \mathrm{sec}$ break after $10 \mathrm{sec}$ of sonication) at an amplitude of $45 \%$ in an ice-water bath. Instead of immediate lysis of bacterial cells, frozen suspensions can be stored at $-80^{\circ} \mathrm{C}$ for several month. The bacterial lysate was centrifuged for $30 \mathrm{~min}$ at $15000 \mathrm{rpm}$ (about $27.000 \mathrm{x}$ g, SS34 rotor; Sorvall ${ }^{\circledR}$ ) and the resulting supernatant was subjected to affinity chromatography. Proteins containing a His-tag were bound to nickel (Ni-) NTAagarose (Qiagen) while glutathione-Sepharose 4B (Amersham) or GST Bind Resin (Novagen) was used to purify GST-fused proteins. After washing (usually three times), recombinant 
proteins were eluted with imidazole or reduced glutathione, respectively. The concentration of imidazole was dependent on the protein while reduced glutathione was used in a concentration of $10 \mathrm{mM}$ (10x stock solution: $500 \mathrm{mM}$ Tris- $\mathrm{HCl} \mathrm{pH}$ 8.0, $1.5 \mathrm{M} \mathrm{NaCl}, 100 \mathrm{mM}$ glutathione). Binding to the affinity matrix and washing steps were performed in solution (batch). The resin was then packed into a column where the elution took place.

Depending on the protein additional purification steps such as size exclusion or ion-exchange chromatography were performed. Size exclusion chromatography (gel filtration) separates molecules according to differences in their size. The gel filtration matrix consists of porous beads with a well-defined range of pore sizes. Molecules diffuse in and out of the pores. Small molecules diffuse further into the matrix and therefore they elute later than large molecules that can only partially diffuse into the matrix or are totally excluded. Separation in ion exchange chromatography depends on the reversible adsorption of charged molecules to immobilized ion exchange groups of opposite charge. Molecules vary in their interaction with the ion exchanger due to differences in their charges, charge densities and distribution of charge on their surfaces. These interactions can be controlled by varying conditions such as ionic strength and $\mathrm{pH}$.

Before freezing $250 \mathrm{mM}$ sucrose were generally added to the purified proteins.

\subsubsection{Expression and purification of nuclear transport cargoes}

\subsection{DFF40/DFF45 complexes}

Epitope-tagged DFF40/DFF45 complexes were generated as follows: DFF40 and DFF45 were coexpressed in E. coli BL21 (DE3). The cultures were grown in SOB medium at $37^{\circ} \mathrm{C}$ to an optical density of 0.9 at $600 \mathrm{~nm}$. After shifting the temperature to $18^{\circ} \mathrm{C}$ bacterial protein expression was induced with $0.2 \mathrm{mM}$ IPTG and the cultures were grown for 18-20 hours. After centrifugation, the collected cells were resuspended in buffer A $\left(50 \mathrm{mM} \mathrm{NaH} \mathrm{PO}_{4} \mathrm{pH} 8.0\right.$, $300 \mathrm{mM} \mathrm{NaCl}, 2 \mathrm{mM}$ BME), lysed by sonication and the recombinant DFF40/DFF45 complexes were bound to Ni-NTA-agarose (Qiagen) overnight at $4^{\circ} \mathrm{C}$ in the presence of $20 \mathrm{mM}$ imidazole. Afterwards, the matrix was washed three times with buffer A containing $50 \mathrm{mM}$ imidazole. EGFP-DFF40/His-DFF45 was eluted with $100 \mathrm{mM}$ imidazole in buffer A and subsequently applied to a Superdex 200 10/300 GL column (gel filtration) equilibrated with transport buffer (20 mM HEPES-KOH, pH 7.4, $110 \mathrm{mM}$ potassium acetate, $5 \mathrm{mM}$ magnesium acetate, $0.5 \mathrm{mM}$ EGTA, $2 \mathrm{mM}$ dithiothreitol, $250 \mathrm{mM}$ sucrose). To elute the GST-DFF40/His-DFF45 and HisDFF40/GST-DFF45 complexes from the Ni-NTA-agarose $300 \mathrm{mM}$ of imidazole in buffer A were used. Eluted protein was applied to glutathione-Sepharose, and buffer A was added to a total volume of about $30 \mathrm{ml}$. After $2-3 \mathrm{~h}$ incubation at $4{ }^{\circ} \mathrm{C}$ the matrix was washed with buffer $\mathrm{A}$ 
and the recombinant DFF complexes were eluted with reduced glutathione (in $50 \mathrm{mM}$ Tris $\mathrm{pH} 8.0,150 \mathrm{mM} \mathrm{NaCl})$.

\subsection{DFF subunits}

GST-DFF45 and GST-DFF45(aa296-331) were expressed in E. coli BL21 (DE3) in LB medium for 2 hours at $30^{\circ} \mathrm{C}$ with $0.2 \mathrm{mM}$ IPTG and were subsequently purified on glutathione-Sepharose according to the manufacturer's instruction. Briefly, after centrifugation the cells were resuspended in GST-Bind/Wash buffer $\left(4.3 \mathrm{mM} \mathrm{Na}_{2} \mathrm{HPO}_{4}, 1.47 \mathrm{mM} \mathrm{KH} \mathrm{KO}_{4}, 137 \mathrm{mM} \mathrm{NaCl}\right.$, $2.7 \mathrm{mM} \mathrm{KCl}, \mathrm{pH}$ 7.3), lysed by sonication and the GST-fused protein was bound to glutathioneSepharose for 2-3 $\mathrm{h}$ at $4^{\circ} \mathrm{C}$. After washing three times with GST-Bind/Wash buffer bound proteins were eluted with reduced glutathione (in $50 \mathrm{mM}$ Tris $\mathrm{pH} 8.0,150 \mathrm{mM} \mathrm{NaCl}$ ).

\subsection{Caspase-3}

His-caspase-3 was expressed in S. cerevisiae (ENY.WA-4D) as described in Goebel et al. (Goebel et al., 2007). A single colony was grown in SCD-Leu medium at $30^{\circ} \mathrm{C}$ overnight and then diluted 1:10 in SCD-Leu. After further incubation for $9 \mathrm{~h}$ at $30^{\circ} \mathrm{C}$ the culture was used to inoculate 6x $200 \mathrm{ml}$ SCGL-Leu. At an optical density of 1.0-1.3 at $600 \mathrm{~nm}$ YPGLA medium was added to the cultures. After growing for $3 \mathrm{~h}$ at $30^{\circ} \mathrm{C}$ the protein expression was induced with $2 \%$ galactose for $6 \mathrm{~h}$. The harvested yeast cells were washed two times with $0.1 \mathrm{M}$ Tris- $\mathrm{SO}_{4} \mathrm{pH} 9.2$, resuspended in $50 \mathrm{mM}$ Tris- $\mathrm{HCl} \mathrm{pH} 7.9$ and $400 \mathrm{mM} \mathrm{NaCl}$ and lysed by vortexing with glass beads. Further affinity purification was performed on nickel NTA-agarose according to the QIAexpressionist (protein purification handbook, Qiagen). The purification of His-tagged caspase-3 used in this study was done by Nadja Bleicher (former diploma student in the Department of Molecular Biology, University of Göttingen).

\subsection{Nucleoplasmin}

The expression plasmid for GST-nucleoplasmin was kindly provided by Prof. Pieler (Developmental Biochemistry, University of Göttingen). GST-nucleoplasmin was expressed in E. coli BL21 (DE3) for 3 hours at $30^{\circ} \mathrm{C}$ with $1 \mathrm{mM}$ IPTG and was purified on glutathioneSepharose (see above).

\subsection{Expression and purification of import receptors and supplementary factors}

The expression plasmids for importin $\alpha$, importin $\beta$, transportin importin 5 , importin 7 and importin 13 were kindly provided by Dirk Görlich, José-Manuel Mingot and Stefan Jäkel 
(ZMBH, Heidelberg, Germany). The expression plasmid for importin 9 was kindly provided by Ulrike Kutay (Institute for Biochmemistry, ETH Zürich). The import factors were expressed in E. coli BL21 (DE3) or E. coli JM109 as described in the literature indicated and were purified on nickel NTA-agarose, followed occasionally by chromatography on Superdex 200 (depending on the purity of the transport receptors): Хеnopus importin $\alpha 1$ (Görlich et al., 1994), human importin $\beta$ (Kutay et al., 1997b), transportin (Izaurralde et al., 1997), Xenopus importin 7, importin 5 (Jakel and Görlich, 1998), importin 9 (Muhlhausser et al., 2001) and importin 13 (Mingot et al., 2001).

\subsection{Ran and NTF2}

N-terminally His-tagged Ran (wild-type and Q69L) was expressed (2 mM IPTG, 3 hours, 30 ${ }^{\circ} \mathrm{C}$ ) in E. coli JM109 from pQE32 (cloned as a SphI-HindIII fragments) and purified essentially as described by Ribbeck et al. (Ribbeck et al., 1998).

Briefly, Ran (wild-type) bacterial pellets were resuspended in $50 \mathrm{mM}$ potassium phosphate $\mathrm{pH}$ 7.0, $200 \mathrm{mM} \mathrm{NaCl}, 5 \mathrm{mM} \mathrm{MgCl}_{2}$, sonicated, centrifuged, and then $1 \mathrm{mM}$ GDP was added. Ran was bound on Ni-NTA-agarose, and was eluted with $50 \mathrm{mM}$ potassium phosphate $\mathrm{pH}$ 7.0, $5 \mathrm{mM}$ $\mathrm{MgCl}_{2}, 150 \mathrm{mM}$ imidazole. The fractions containing Ran were pooled, diluted 5-fold in $5 \mathrm{mM}$ potassium phosphate $\mathrm{pH} 7.0,0.5 \mathrm{mM} \mathrm{MgCl}_{2}, 5 \%$ glycerol, and were then applied to a $1 \mathrm{ml}$ Mono S column (GE Healthcare). Ran was eluted from the Mono S column with a linear gradient from buffer $\mathrm{B}(20 \mathrm{mM}$ potassium phosphate $\mathrm{pH} 7.0,0.5 \mathrm{mM} \mathrm{MgCl} 2,5 \%$ glycerol) to buffer C (0.5 M potassium phosphate $\left.\mathrm{pH} 7.0,0.5 \mathrm{mM} \mathrm{MgCl}_{2}\right)$. Thereby, RanGDP elutes earlier than RanGTP.

In contrast, RanQ69L(GTP) bacterial pellets were resuspended in $50 \mathrm{mM}$ HEPES-KOH pH 7.0, $100 \mathrm{mM} \mathrm{NaCl}, 5 \mathrm{mM} \mathrm{MgCl} 2,5 \mathrm{mM} \mathrm{BME}$ ), sonicated, centrifuged, and bound on Ni-NTAagarose. The pre-bound nucleotide was removed by washing for 1 hour with $50 \mathrm{mM}$ HEPES$\mathrm{KOH} \mathrm{pH}$ 7.0, $200 \mathrm{mM} \mathrm{NaCl}, 5 \mathrm{mM} \mathrm{BME}$, and RanQ69L was then reloaded with GTP in the presence of $100 \mu \mathrm{M}$ GTP in $50 \mathrm{mM}$ HEPES-KOH pH 7.0, $200 \mathrm{mM} \mathrm{NaCl}, 5 \mathrm{mM} \mathrm{MgCl} 2,5 \mathrm{mM}$ BME (incubation again for 1 hour). After elution with $50 \mathrm{mM}$ HEPES-KOH pH 7.0, $200 \mathrm{mM}$ $\mathrm{NaCl}, 5 \mathrm{mM} \mathrm{MgCl}$, $5 \mathrm{mM}$ BME, $250 \mathrm{mM}$ imidazole the RanQ69L(GTP) fractions were pooled, supplemented with $250 \mathrm{mM}$ sucrose and frozen in liquid nitrogen.

NTF2 was expressed and purified essentially as described (Kutay et al., 1997b; Ribbeck et al., 1998). Briefly, untagged NTF2 was expressed from a pET expression vector in E. coli BL21 (DE3) pLysS (Novagen) for $2 \mathrm{~h}$ at $30^{\circ} \mathrm{C}$ with $1 \mathrm{mM}$ IPTG (Kent et al., 1996). NTF2 was 
precipitated from the bacterial lysate with ammonium sulfate (50\% saturation), the pellet was dissolved in $250 \mathrm{ml}$ buffer D (50 mM Tris pH 8.0, $2 \mathrm{mM}$ DTT) and NTF2 was then bound to Q Sepharose FF (HiLoad Q Sepharose Fast Flow column, GE Healthcare) equilibrated in buffer D. NTF2 eluted in the $\mathrm{NaCl}$ gradient (buffer E: $50 \mathrm{mM}$ Tris $\mathrm{pH}$ 8.0, $500 \mathrm{mM} \mathrm{NaCl}, 2 \mathrm{mM}$ DTT) at about $230 \mathrm{mM} \mathrm{NaCl}$. Final purification was performed on Superdex 75 (GE Healthcare) where NTF2 eluted at a position expected for the homodimer. The purification of NTF2 was done by Marc Bäuerle (former PhD student in the Department of Molecular Biology, University of Göttingen).

\subsection{Import factors}

C-terminally $\mathrm{His}_{6}$-tagged importin $\boldsymbol{\alpha}$ and importin $\boldsymbol{\alpha E 3 8 8 R}$ were expressed in E. coli JM109 from pQE70 (cloned as a SphI-BamHI fragment). JM109 cells were freshly transformed, grown overnight at $37^{\circ} \mathrm{C}$ and next day the whole plate was rinsed with $\mathrm{LB}$ medium. The resulting bacterial suspension was used to inoculate LB medium containing $2 \%$ glucose that was again incubated overnight at $37^{\circ} \mathrm{C}$. The culture was scaled up to 2-3 1 of LB medium and was grown to an optical density of 0.9 at $600 \mathrm{~nm}$. Expression was induced with $2 \mathrm{mM} \mathrm{IPTG}$ at $26^{\circ} \mathrm{C}$ for 3 hours. The bacterial cells were pelleted and resuspended in sonication buffer ( $50 \mathrm{mM}$ Tris-HCl $\mathrm{pH}$ 7.5, $500 \mathrm{mM} \mathrm{NaCl}, 5 \%$ glycerol, and $2 \mathrm{mM} \mathrm{BME}$ ), freeze-thawed and lysed by sonication. After centrifugation, the protein was bound to Ni-NTA-agarose at $4^{\circ} \mathrm{C}$ overnight. The affinity matrix was washed three times with sonication buffer containing $20 \mathrm{mM}$ imidazole and the resin was used to build up a column. Importin $\alpha$ was eluted with $100 \mathrm{mM}$ imidazole in $50 \mathrm{mM}$ Tris$\mathrm{HCl}, \mathrm{pH} 7.5$ and $300 \mathrm{mM} \mathrm{NaCl}$ and dialyzed against buffer $\mathrm{F}$ (50 mM Tris- $\mathrm{HCl} \mathrm{pH}$ 7.5, $200 \mathrm{mM}$ $\left.\mathrm{NaCl}, 5 \mathrm{mM} \mathrm{MgCl}_{2}, 2 \mathrm{mM} \mathrm{BME}\right)$. In contrast, importin $\alpha \mathrm{E} 388 \mathrm{R}$ was dialyzed against buffer $\mathrm{G}$ (50 mM Tris- $\mathrm{HCl} \mathrm{pH}$ 7.5, $300 \mathrm{mM} \mathrm{NaCl}, 5 \mathrm{mM} \mathrm{MgCl}, 2 \mathrm{mM} \mathrm{BME}$ ).

Additionally, recombinant human importin $\alpha 2$ used in in vitro import assays was purchased from Calbiochem.

C-terminally $\mathrm{His}_{6}$-tagged importin $\boldsymbol{\beta}$ and $\mathrm{N}$-terminally $\mathrm{His}_{6}$-tagged transportin were expressed in E. coli JM109 from pQE60 and from pQE32, respectively. For expression a pre-inoculum was grown overnight in $200 \mathrm{ml} \mathrm{LB}$ medium containing $2 \%$ glucose and then used to inoculate $600 \mathrm{ml}$ of LB medium. The bacteria were grown at $37^{\circ} \mathrm{C}$ until an optical density of $1(600 \mathrm{~nm})$ was reached, then $800 \mathrm{ml}$ of ice-cold LB medium containing 4\% ethanol were added, and the culture was shifted to $21^{\circ} \mathrm{C}$. After the temperature had equilibrated $0.5 \mathrm{mM}$ IPTG was added and the protein was expressed for 4 hours. Cells were collected by centrifugation, resuspended in 
sonication buffer (see above), and lysed by sonication. The protein was bound to Ni-NTAagarose, eluted with $200 \mathrm{mM}$ and $400 \mathrm{mM}$ imidazole in sonication buffer and further purified by chromatography on Superdex 200 equilibrated with buffer F.

Recombinant human importin $\beta$ used in in vitro import assays was also purchased from Calbiochem.

Purified importin $\boldsymbol{\beta} \Delta \mathbf{1 - 1 2 7}$ (importin $\beta C 127$ ) and importin $\boldsymbol{\beta \Delta 2 1 0}$ (importin $\beta C 210$ ) were a kind gift of Daniel Wohlwend and Ralf Ficner (Department of Molecular Structural Biology, University of Göttingen).

N-terminally His $_{6}$-tagged importin 5 was expressed in E. coli JM109 from pQE32. For expression a pre-inoculum was grown overnight in $100 \mathrm{ml} 2 \mathrm{xYT}$ medium containing $30 \mathrm{mM}$ $\mathrm{K}_{2} \mathrm{HPO}_{4} \mathrm{pH} 7.0$ and $2 \%$ glucose and was then used to inoculate $900 \mathrm{ml}$ of $2 \times \mathrm{xT}$ medium with 30 $\mathrm{mM} \mathrm{K}_{2} \mathrm{HPO}_{4}$. The culture was grown to an optical density of 1.0 at $600 \mathrm{~nm}$ and 11 cold medium containing $4 \%$ ethanol was added. Importin 5 was expressed for $4 \mathrm{~h}$ at $17{ }^{\circ} \mathrm{C}$ with $0.25 \mathrm{mM}$ IPTG and purified as described for importin $\beta$.

N-terminally His ${ }_{10}$-tagged importin 7 was expressed in E. coli M15 (pREP4) from pQE80. For expression a pre-inoculum was grown overnight in $100 \mathrm{ml} 2 \mathrm{xYT}$ medium containing $30 \mathrm{mM}$ $\mathrm{K}_{2} \mathrm{HPO}_{4} \mathrm{pH} 7.0$ and $2 \%$ glucose and was then used to inoculate $900 \mathrm{ml}$ of $2 \times \mathrm{xT}$ medium with 30 $\mathrm{mM} \mathrm{K}_{2} \mathrm{HPO}_{4}$. The culture was grown at $37^{\circ} \mathrm{C}$ to an optical density of $0.7(600 \mathrm{~nm})$, was shifted to $26^{\circ} \mathrm{C}$ and further incubated to an optical density of 2.0. After addition of $112 \mathrm{xYT}$ medium containing $30 \mathrm{mM} \mathrm{K} 2 \mathrm{HPO}_{4}$ and 4\% ethanol importin 7 was expressed with $0.05 \mathrm{mM}$ IPTG at $13^{\circ} \mathrm{C}$ overnight. The culture was cooled down on ice and $1 \mathrm{mM}$ PMSF was added. After centrifugation, cells were resuspended in buffer G containing 5\% glycerol, lysed by sonication, centrifuged and the protein was bound to Ni-NTA-agarose. The matrix was washed with $20 \mathrm{mM}$ Tris- $\mathrm{HCl} \mathrm{pH} 7.5,300 \mathrm{mM} \mathrm{NaCl}$ and $40 \mathrm{mM}$ imidazole, the protein was eluted with 100-200 $\mathrm{mM}$ IPTG and dialyzed against buffer F.

$\mathrm{N}$-terminally His ${ }_{6}$-tagged Importin 9 was expressed in E. coli JM109 from pQE32 (cloned as XmaI fragment). For expression a pre-inoculum was grown overnight in $100 \mathrm{ml} 2 \mathrm{xYT}$ medium containing $30 \mathrm{mM} \mathrm{K}_{2} \mathrm{HPO}_{4} \mathrm{pH} 7.0$ and $2 \%$ glucose. This overnight-culture was then used to inoculate $900 \mathrm{ml}$ of $2 \mathrm{xYT}$ medium with $30 \mathrm{mM} \mathrm{K}_{2} \mathrm{HPO}_{4}$. The culture was grown to an optical density of 1.0 at $600 \mathrm{~nm}$ and 11 cold $2 x Y T$ medium containing $30 \mathrm{mM} \mathrm{K}_{2} \mathrm{HPO}_{4} 2 \%$ ethanol was 
added. Importin 9 was expressed for $3 \mathrm{~h}$ at $20^{\circ} \mathrm{C}$ with $0.5 \mathrm{mM}$ IPTG and purified as described for importin $\beta$.

$\mathrm{N}$-terminally $\mathrm{His}_{6}$-tagged importin 13 was expressed in E. coli JM109 from pQE80 (cloned as a BamHI-HindIII fragment). For expression a pre-inoculum was grown overnight in $50 \mathrm{ml} 2 \mathrm{xYT}$ medium containing $30 \mathrm{mM} \mathrm{K} \mathrm{HPO}_{4}$ and $2 \%$ glucose and was scaled up to $700 \mathrm{ml} 2 \mathrm{xYT}$ containing $30 \mathrm{mM} \mathrm{K} \mathrm{HPO}_{4}$ and $2 \%$ glycerol. The culture was grown at $37^{\circ} \mathrm{C}$ to an optical density of $1(600 \mathrm{~nm})$, then 1.21 cold 2 xYT medium were added and the culture was shifted to $16^{\circ} \mathrm{C}$. At an optical density of about 0.75 expression of importin 13 was induced with $0.1 \mathrm{mM}$ IPTG at $16^{\circ} \mathrm{C}$ overnight $(18 \mathrm{~h})$. The cells were centrifuged, resuspended in $50 \mathrm{mM}$ Tris- $\mathrm{HCl}$ $\mathrm{pH}$ 7.5, $500 \mathrm{mM} \mathrm{NaCl}, 10 \mathrm{mM}$ magnesium acetate, $5 \mathrm{mM} \mathrm{BME}$, and $5 \%$ glycerol and lysed by sonication. The protein was bound on Ni-NTA-agarose and eluted with $250 \mathrm{mM}$ imidazole in resuspension buffer and further purified on a Superdex 200 equilibrated with $50 \mathrm{mM}$ Tris- $\mathrm{HCl}$ $\mathrm{pH} 7.5,100 \mathrm{mM} \mathrm{NaCl}$ and $3 \mathrm{mM}$ magnesium acetate.

\subsubsection{In vitro transcription-translation}

In vitro transcription-translation of the six different Xenopus laevis importin $\alpha$ variants (pJW158, pJW160, pJW224, pJW226, pJW227, pJW228; kindly provided by Jörg Wischnewski and Thomas Pieler, Department of Developmental Biochemistry, University of Göttingen) was performed from the corresponding SP6 promoter constructs (pCS2+ and pCS2+MT). Using the $\mathrm{T}_{\mathrm{N}} \mathrm{T}$ coupled reticulocyte lysate system (Promega) according to the manufacturer's instructions the proteins were labeled with $\left[{ }^{35} \mathrm{~S}\right]$ methionine (Amersham). Reactions were performed at $30^{\circ} \mathrm{C}$ for 2 hours in a $12.5 \mu \mathrm{l}$ volume and the samples were then directly used for GST-pull down assays.

\subsubsection{Activation of DFF40 with caspase-3}

\subsection{DNA cleavage assay}

The DNA cleavage assay was used to analyze the functionality of recombinant purified DFF40/DFF45 complexes. Functional DFF complexes can be activated by caspase-3 leading to the release of nucleolytically active DFF40 which was detected by the cleavage of plasmid DNA. Increasing amounts of recombinant, purified epitope-tagged DFF40/DFF45 complexes dialyzed against activation buffer $(10 \mathrm{mM}$ HEPES-KOH pH 7.0, $50 \mathrm{mM} \mathrm{NaCl}, 20 \%$ glycerin, $2 \mathrm{mM}$ $\mathrm{MgCl}_{2}, 5 \mathrm{mM}$ DTT) were incubated with recombinant purified His-caspase-3 (in activation buffer), and $1 \mu \mathrm{g}$ circular plasmid DNA, $5 \mu \mathrm{M} \mathrm{MgCl}$ and $4.4 \mu \mathrm{M}$ DTT for $2 \mathrm{~h}$ at $37^{\circ} \mathrm{C}$ in buffer G (20 mM HEPES-KOH pH 7.5, $10 \mathrm{mM} \mathrm{KCl,} 1$ mM EDTA, 1 mM EGTA, 1 mM DTT) in a 
total volume of $50 \mu \mathrm{l}$. The reactions were stopped on ice and $\mathrm{NaCl}$ was added to a final concentration of $1 \mathrm{M}$. The DNA was extracted by adding $50 \mu \mathrm{l}$ phenol and $50 \mu 1$ chloroform/isoamylalcohol (24:1), vortexing and centrifugation for $5 \mathrm{~min}$ at $13000 \mathrm{rpm}$. The DNA containing aqueous supernatant was then mixed with $300 \mu \mathrm{l}$ ethanol and frozen for $1 \mathrm{~h}$ at $-80^{\circ} \mathrm{C}$ for DNA precipitation. After centrifugation for $15 \mathrm{~min}$ at $13000 \mathrm{rpm}$ and $4^{\circ} \mathrm{C}$ the DNA pellet was washed with $200 \mu 170 \%$ ethanol, dried in the Speed Vac centrifuge and dissolved in $20 \mu 1$ TE buffer (10x TE: $100 \mathrm{mM}$ Tris-HCl pH 8.0, $10 \mathrm{mM}$ ETDA). $10 \mu 1$ of each sample were analyzed by agarose gel electrophoresis on a 1\% agarose gel.

\subsection{Activation of immobilized DFF40}

Immobilized GST-DFF40/His-DFF45 was incubated with His-tagged caspase-3 in activation buffer for $30 \mathrm{~min}$ at $30^{\circ} \mathrm{C}$. The beads were washed twice in activation buffer and once in buffer $\mathrm{F}$ containing 5\% glycerol. The immobilized activated GST-DFF40 was subjected to in vitro binding studies (chapter 4.2.2.8.1).

\subsubsection{GST-pull down assays}

\subsection{In vitro binding studies with bacterially expressed proteins}

GST-fusion proteins immobilized on glutathione-Sepharose (see chapter 4.2.2.5) were used as affinity matrix for in vitro binding experiments. Appropriate amounts of this affinity matrix were incubated for $3 \mathrm{~h}$ at $4^{\circ} \mathrm{C}$ with either bacterial lysates containing expressed import receptors or the corresponding purified recombinant import factor in buffer $\mathrm{F}$ containing 5\% glycerol. The binding experiments were performed in the absence or presence of $2 \mu \mathrm{M}$ RanQGTP. After washing three times with ice-cold buffer $\mathrm{F}$ supplemented with 5\% glycerol, the beads were boiled in SDS-PAGE sample buffer and analyzed by SDS-PAGE.

\subsection{GST-pull down assays after overexpression of DFF subunits in HeLa cells}

To analyze whether fluorescently tagged DFF40 and DFF45 subunits interact with each other in cotransfected HeLa cells GST-pull down assays were performed based on the method described in Korn et al. (Kent et al., 1996). HeLa P4 cells were cultured in $10 \mathrm{~cm}$-dishes and were cotransfected with DFF subunits fused to EGFP-GST and RFP, respectively (see chapter 4.2.3.2). $24 \mathrm{~h}$ post transfection, the cells were washed twice with cold PBS $(140 \mathrm{mM} \mathrm{NaCl}$, $2.7 \mathrm{mM} \mathrm{KCl}, 1.5 \mathrm{mM} \mathrm{KH}_{2} \mathrm{PO}_{4}$, and $9 \mathrm{mM} \mathrm{Na}_{2} \mathrm{HPO}_{4} \mathrm{pH}$ 7.4) and were subsequently harvested in $1 \mathrm{ml}$ PBS using a cell scraper. Cells were pelleted (5 min, $1000 \mathrm{rpm})$, resuspended in $1 \mathrm{ml}$ buffer H (20 mM HEPES-NaOH pH 7.4, 100 mM NaCl, 1 mM EDTA, 10\% glycerol and 5 mM DTT) 
and lysed by sonication (5x $3 \mathrm{sec}$, amplitude 45\%). After addition of Triton X-100 to a final concentration of $0.01 \%$ the cell lysates were incubated for $5 \mathrm{~min}$ at $4{ }^{\circ} \mathrm{C}$ (rotating) and were subsequently centrifuged for $30 \mathrm{~min}$ at $14000 \mathrm{rpm}$ and $4^{\circ} \mathrm{C}$. Afterwards, the supernatants were applied on glutathione-Sepharose $(\sim 40 \mu$, equilibrated with buffer $\mathrm{H})$ and were incubated at $4^{\circ} \mathrm{C}$ overnight. After washing three times with buffer $\mathrm{H}$ (rotation for $10 \mathrm{~min}$ ) the beads were boiled in SDS-PAGE sample buffer and analyzed by SDS-PAGE.

\subsubsection{Cell biological methods}

\subsubsection{Cell culture}

\subsection{Maintenance of eukaryotic cells}

HeLa cells were grown grown in modified Eagle's medium (MEM, Biochrom). HeLa P4 cells (Korn et al., 2002) were cultured in Dulbecco's modified Eagle's medium additionally supplemented with $2 \mathrm{mM}$ glutamine (DMEM, GIBCO). Both media were also supplemented with $10 \%(\mathrm{v} / \mathrm{v})$ fetal bovine serum (FBS, Biochrom) and antibiotics. Cells were maintained in a humidified incubator with $5 \% \mathrm{CO}_{2}$ atmosphere at $37^{\circ} \mathrm{C}$.

\subsection{Passaging of eukaryotic cells}

Confluent HeLa and HeLa P4 cell cultures were split every three days about 1:10 and 1:20, respectively (a single culture was not subcultured more than 25 times). After removing the medium, the cells were washed once with PBS which was then replaced by trypsin/EDTA $\left[0.05 \%(\mathrm{w} / \mathrm{v})\right.$ trypsin, $0.02 \%(\mathrm{w} / \mathrm{v})$ EDTA in PBS]. The cells were incubated at $37^{\circ} \mathrm{C}$ until they were detached from the culture flask surface and then five times the volume of the corresponding medium was added to inhibit the proteolytic reaction mediated by trypsin. After singularizing by pipetting up and down, the cells were centrifuged $5 \mathrm{~min}$ at $1000 \mathrm{~g}$, washed once with PBS and resuspended in fresh medium. For subsequent experiments such as transfection studies and nuclear import assays the cells were plated $24 \mathrm{~h}$ prior to the experiment as follows: $1.2 \times 10^{5}$ cells/ 12 well, $3 \times 10^{5}$ cells/ 6 well $\left(2 \times 10^{5}\right.$ cells/ 6 well in case of plating the cells 48 h prior to nuclear import assays) and $2.7 \times 10^{6}$ cells/ $10 \mathrm{~cm}$ dish. For in vitro nuclear import assays and fluorescence microscopy after in vivo transfection the cells were grown on glass cover slips $(\varnothing$ $10 \mathrm{~mm})$.

\subsection{Preservation and storage}

Eukaryotic cells were stored in liquid nitrogen and the cryoprotective agent DMSO was added to minimize cell damage caused by freezing. $3 \times 10^{6}$ cells per $\mathrm{ml}$ freezing medium $(20 \% \mathrm{FBS} ; 10 \%$ 
DMSO in the respective medium) were slowly frozen overnight at $-80^{\circ} \mathrm{C}$ (the cryo-tubes were wrapped in cellulose and stored in a polystyrene box). Thereby, cell damage caused by ice crystals is strongly reduced because water can move out of the cells before it freezes. The next day, the cells were frozen in liquid nitrogen. To recover frozen cells, they were thawed quickly in a $37^{\circ} \mathrm{C}$ water bath and diluted in pre-warmed medium.

\subsubsection{Transient transfection of eukaryotes}

Chemical transfection with liposome-like vesicles was used to transiently transfect eukaryotic cells with recombinant plasmid DNA. Thereby, the DNA is coated with synthetic cationic lipids and these artificial membrane vesicles fuse with the plasma membranes of the cells. $1.2 \times 10^{5}$ HeLa P4 cells were seeded per well in a 12 well-plate $24 \mathrm{~h}$ prior to transfection. Chemical transfection with $0.3 \mu \mathrm{g}$ DNA was performed with the Effectene ${ }^{\mathrm{TM}}$ Transfection Reagent (Qiagen) according to the manufacturer's instructions. Briefly, the DNA was first condensed with the Enhancer and then Effectene was added to generate condensed Effectene-DNA complexes and to form micelle structures that can be internalized by the cells. The EffecteneDNA complexes were mixed with the respective medium and were directly added to the cells. $24 \mathrm{~h}$ post transfection the cells were fixed with 3\% paraformaldehyde in PBS for 15 minutes, the nuclei were visualized with Hoechst33258 (Molecular Probes), and the cells were subjected to fluorescence microscopy. In case of interaction experiments (GST-pull down assays) 2.7x10 cells were seeded per $10 \mathrm{~cm}$ dish, were transfected with $2 \mu \mathrm{g}$ DNA and were harvested $24 \mathrm{~h}$ after transfection (chapter 4.2.2.8.2).

\subsubsection{In vitro nuclear import assays}

Import reactions were based on the method established by Adam et al. (Adam et al., 1990) and were performed essentially as previously described (Jakel and Görlich, 1998). This assay allows reconstitution of nuclear import events by using recombinant purified transport factors without the interference of endogenous importins.

HeLa cells were grown on $10 \mathrm{~mm}$ glass coverslips to $40-80 \%$ confluence. The cholesterol-rich plasma membrane was selectively permeabilized with the non-ionic detergent digitonin while the nuclear membrane stayed intact. The cells were incubated with $40 \mu \mathrm{g} / \mathrm{ml}$ digitonin (Calbiochem) in transport buffer (20 mM HEPES-KOH, pH 7.4, $110 \mathrm{mM}$ potassium acetate, $5 \mathrm{mM}$ magnesium acetate, 0,5 mM EGTA, $2 \mathrm{mM}$ dithiothreitol, $250 \mathrm{mM}$ sucrose) for 5-10 $\mathrm{min}$ on ice. For cytosol depletion, the cells were kept on ice and were washed three times (for $1 \mathrm{~min}, 5 \mathrm{~min}$ and $10 \mathrm{~min}$ ) with ice-cold transport buffer. The permeabilized cells were incubated at $37^{\circ} \mathrm{C}$ with $20 \mu 1$ of a 
transport reaction mix containing $0.35 \mu \mathrm{M}$ substrate, either $10 \mu \mathrm{l}$ of reticulocyte lysate (Promega) as general source of import receptors or $0.5 \mu \mathrm{M}$ recombinant importin and an energyregenerating system $(0.5 \mathrm{mM}$ ATP, $0.5 \mathrm{mM}$ GTP, $10 \mathrm{mM}$ creatine phosphate, $50 \mu \mathrm{g} / \mathrm{ml}$ creatine kinase) in transport buffer. Performing reconstitution experiments with recombinant transport factors a Ran mix [3 $\mu \mathrm{M} \operatorname{Ran}(\mathrm{GDP})$ and $0.5 \mu \mathrm{M}$ NTF2] was added to the transport reaction mix to generate a RanGTP gradient across the nuclear membrane. For negative controls the assay was done in the absence of reticulocyte lysate or recombinant transport factors. HeLa cells were fixed with 3\% paraformaldehyde for $15 \mathrm{~min}$ and were mounted by using Vectashield with DAPI (Vector Laboratories). Import reactions were visualized by fluorescence microscopy with a Zeiss microscope (Axioskop 20) using a 40x objective lens (Plan Neofluar).

\subsection{Inhibition experiments}

Import reactions under inhibitory conditions were carried out as follows: Prior to addition of the transport reaction mixture the cells were pre-incubated with $50 \mu \mathrm{g} / \mathrm{ml}$ wheat germ agglutinin (WGA) for $15 \mathrm{~min}$ at room temperature and were then washed three times with tranport buffer. WGA binds to $\mathrm{N}$-acetylglucosylated nucleoporins, thereby inhibiting receptor-mediated active transport processes through the nuclear pore complexes while passive diffusion remains unaffected (Finlay et al., 1987; Yoneda et al., 1987). Whether the blocked nuclear transport is caused by competition of WGA with import receptors or by steric interference is not known.

For energy depletion, the energy-regenerating system was replaced with apyrase $(100 \mathrm{U} / \mathrm{ml}$; Sigma). Apyrase is an ATP diphosphohydrolase that catalyses the removal of the gamma phosphate from ATP and the beta phosphate from ADP. Additionally, import reactions were also carried out at $4^{\circ} \mathrm{C}$ instead of $37^{\circ} \mathrm{C}$. 


\section{Summary}

One of the biochemical hallmarks of apoptosis is internucleosomal DNA fragmentation mediated by the endonuclease DFF40. In proliferating cells DFF40 is expressed in the presence of DFF45 which has a dual function as chaperone and inhibitor of DFF40. The DFF complex is translocated into the nucleus where DFF40 is activated during apoptosis due to caspase-3mediated cleavage of DFF45.

In this study, we report that transition of the human DFF complex through the nuclear pore is signal-dependent, energy-dependent, and mediated by nuclear transport receptors. The DFF complex is imported into the nucleus via the classical importin $\alpha / \beta$-pathway. Among the importin $\alpha$ isoforms Xenopus laevis importin $\alpha 1$ showed the highest binding competence indicating that the DFF complex preferably interacts with importin $\alpha$ subtypes belonging to the Rch1 subclass. The sequence elements essential for recognition of the DFF complex by importin $\alpha / \beta$ were mapped to the $C$-terminal regions of both subunits. At least one basic cluster in each subunit, DFF40 (RLKRK) and DFF45 (KRAR), is required for nuclear import of the DFF complex.

Moreover, the basic cluster KRAR was also identified as key element for the nuclear transport of monomeric DFF45. Further experiments strongly suggest that DFF45 exhibits a classical monopartite NLS that confers nuclear entry via the importin $\alpha / \beta$-pathway. In addition, the Cterminal tail (amino acids 301-338) of DFF40 was sufficient for nuclear accumulation of a normally cytoplasmic protein suggesting that DFF40 harbors an independent NLS. In contrast to the DFF complex activated DFF40 binds not only to importin $\alpha / \beta$ but is also recognized by importin $\beta$. However, nuclear transport of active DFF40 and therefore a functional relevance of these interactions could not be demonstrated.

The fact that both DFF subunits contain NLSs suggests that these signals independently confer nuclear import of the DFF complex. Nevertheless, the interaction between importin $\alpha / \beta$ and the DFF complex was much stronger than binding of importin $\alpha / \beta$ to monomeric DFF45 or active DFF40, respectively. Furthermore, importin $\alpha / \beta$ binding to the DFF complex was almost completely abolished when the C-terminal tail of either DFF40 or DFF45 was deleted. This indicates a substantial importin $\alpha / \beta$ binding cooperativity. Therefore, we propose that DFF40 and DFF45 simultaneously interact with one importin $\alpha / \beta$ heterodimer. The binding of importin $\alpha / \beta$ to the DFF complex was impaired when an importin $\alpha$ minor binding site mutant was used suggesting that the interaction is mediated by a bipartite cNLS. Based on these data, we propose the following model for binding of importin $\alpha / \beta$ to the DFF complex: The essential basic clusters in DFF40 (RLKRK) and DFF45 (KRAR) form an intermolecular bipartite cNLS recognized by 
importin $\alpha / \beta$. The monopartite cNLS of DFF45 (KRAR) thereby presumably contacts the major binding site of importin $\alpha / \beta$ while two adjacent positively charged amino acids of the basic stretch of DFF40 (RLKRK) occupy the minor binding site of importin $\alpha$. 


\section{References}

Abel, F., Sjoberg, R.M., Ejeskar, K., Krona, C. and Martinsson, T. (2002) Analyses of apoptotic regulators CASP9 and DFFA at 1P36.2, reveal rare allele variants in human neuroblastoma tumours. Br J Cancer, 86, 596-604.

Abel, F., Sjoberg, R.M., Krona, C., Nilsson, S. and Martinsson, T. (2004) Mutations in the N-terminal domain of DFF45 in a primary germ cell tumor and in neuroblastoma tumors. Int J Oncol, 25, 1297-1302.

Acehan, D., Jiang, X., Morgan, D.G., Heuser, J.E., Wang, X. and Akey, C.W. (2002) Three-dimensional structure of the apoptosome: implications for assembly, procaspase-9 binding, and activation. Mol Cell, 9, 423-432.

Adam, S.A., Marr, R.S. and Gerace, L. (1990) Nuclear protein import in permeabilized mammalian cells requires soluble cytoplasmic factors. J Cell Biol, 111, 807-816.

Akey, C.W. and Radermacher, M. (1993) Architecture of the Xenopus nuclear pore complex revealed by three-dimensional cryo-electron microscopy. J Cell Biol, 122, 1-19.

Algeciras-Schimnich, A., Shen, L., Barnhart, B.C., Murmann, A.E., Burkhardt, J.K. and Peter, M.E. (2002) Molecular ordering of the initial signaling events of CD95. Mol Cell Biol, 22, 207-220.

Andrade, M.A. and Bork, P. (1995) HEAT repeats in the Huntington's disease protein. Nat Genet, 11, $115-116$.

Ashkenazi, A. and Dixit, V.M. (1998) Death receptors: signaling and modulation. Science, 281, 13051308.

Baake, M., Bauerle, M., Doenecke, D. and Albig, W. (2001a) Core histones and linker histones are imported into the nucleus by different pathways. Eur J Cell Biol, 80, 669-677.

Baake, M., Doenecke, D. and Albig, W. (2001b) Characterisation of nuclear localisation signals of the four human core histones. J Cell Biochem, 81, 333-346.

Bayliss, R., Corbett, A.H. and Stewart, M. (2000a) The molecular mechanism of transport of macromolecules through nuclear pore complexes. Traffic, 1, 448-456.

Bayliss, R., Littlewood, T. and Stewart, M. (2000b) Structural basis for the interaction between FxFG nucleoporin repeats and importin-beta in nuclear trafficking. Cell, 102, 99-108.

Bednenko, J., Cingolani, G. and Gerace, L. (2003) Nucleocytoplasmic transport: navigating the channel. Traffic, 4, 127-135.

Benchoua, A., Couriaud, C., Guegan, C., Tartier, L., Couvert, P., Friocourt, G., Chelly, J., Menissier-de Murcia, J. and Onteniente, B. (2002) Active caspase- 8 translocates into the nucleus of apoptotic cells to inactivate poly(ADP-ribose) polymerase-2. J Biol Chem, 277, 34217-34222.

Besnault-Mascard, L., Leprince, C., Auffredou, M.T., Meunier, B., Bourgeade, M.F., Camonis, J., Lorenzo, H.K. and Vazquez, A. (2005) Caspase-8 sumoylation is associated with nuclear localization. Oncogene, 24, 3268-3273. 
Bischoff, F.R., Klebe, C., Kretschmer, J., Wittinghofer, A. and Ponstingl, H. (1994) RanGAP1 induces GTPase activity of nuclear Ras-related Ran. Proc Natl Acad Sci U S A, 91, 2587-2591.

Bischoff, F.R., Scheffzek, K. and Ponstingl, H. (2002) How Ran is regulated. Results Probl Cell Differ, 35, 49-66.

Boatright, K.M., Renatus, M., Scott, F.L., Sperandio, S., Shin, H., Pedersen, I.M., Ricci, J.E., Edris, W.A., Sutherlin, D.P., Green, D.R. and Salvesen, G.S. (2003) A unified model for apical caspase activation. Mol Cell, 11, 529-541.

Boatright, K.M. and Salvesen, G.S. (2003) Mechanisms of caspase activation. Curr Opin Cell Biol, 15, $725-731$.

Bogerd, H.P., Fridell, R.A., Benson, R.E., Hua, J. and Cullen, B.R. (1996) Protein sequence requirements for function of the human T-cell leukemia virus type 1 Rex nuclear export signal delineated by a novel in vivo randomization-selection assay. Mol Cell Biol, 16, 4207-4214.

Boulares, A.H., Zoltoski, A.J., Yakovlev, A., Xu, M. and Smulson, M.E. (2001) Roles of DNA fragmentation factor and poly(ADP-ribose) polymerase in an amplification phase of tumor necrosis factor-induced apoptosis. J Biol Chem, 276, 38185-38192.

Bradford, M.M. (1976) A rapid and sensitive method for the quantitation of microgram quantities of protein utilizing the principle of protein-dye binding. Anal Biochem, 72, 248-254.

Byun, Y., Chen, F., Chang, R., Trivedi, M., Green, K.J. and Cryns, V.L. (2001) Caspase cleavage of vimentin disrupts intermediate filaments and promotes apoptosis. Cell Death Differ, 8, 443-450.

Chai, J., Wu, Q., Shiozaki, E., Srinivasula, S.M., Alnemri, E.S. and Shi, Y. (2001) Crystal structure of a procaspase-7 zymogen: mechanisms of activation and substrate binding. Cell, 107, 399-407.

Chang, D.W., Xing, Z., Capacio, V.L., Peter, M.E. and Yang, X. (2003) Interdimer processing mechanism of procaspase-8 activation. Embo J, 22, 4132-4142.

Charneau, P., Mirambeau, G., Roux, P., Paulous, S., Buc, H. and Clavel, F. (1994) HIV-1 reverse transcription. A termination step at the center of the genome. J Mol Biol, 241, 651-662.

Chelsky, D., Ralph, R. and Jonak, G. (1989) Sequence requirements for synthetic peptide-mediated translocation to the nucleus. Mol Cell Biol, 9, 2487-2492.

Chen, M.H., Ben-Efraim, I., Mitrousis, G., Walker-Kopp, N., Sims, P.J. and Cingolani, G. (2005) Phospholipid scramblase 1 contains a nonclassical nuclear localization signal with unique binding site in importin alpha. J Biol Chem, 280, 10599-10606.

Chook, Y.M. and Blobel, G. (1999) Structure of the nuclear transport complex karyopherin-beta2-Ran x GppNHp. Nature, 399, 230-237.

Cingolani, G., Bednenko, J., Gillespie, M.T. and Gerace, L. (2002) Molecular basis for the recognition of a nonclassical nuclear localization signal by importin beta. Mol Cell, 10, 1345-1353.

Cingolani, G., Petosa, C., Weis, K. and Muller, C.W. (1999) Structure of importin-beta bound to the IBB domain of importin-alpha. Nature, 399, 221-229.

Cohen, G.M. (1997) Caspases: the executioners of apoptosis. Biochem J, 326 ( Pt 1), 1-16. 
Cohen, J.J. and Duke, R.C. (1984) Glucocorticoid activation of a calcium-dependent endonuclease in thymocyte nuclei leads to cell death. J Immunol, 132, 38-42.

Columbaro, M., Mattioli, E., Lattanzi, G., Rutigliano, C., Ognibene, A., Maraldi, N.M. and Squarzoni, S. (2001) Staurosporine treatment and serum starvation promote the cleavage of emerin in cultured mouse myoblasts: involvement of a caspase-dependent mechanism. FEBS Lett, 509, 423-429.

Colussi, P.A., Harvey, N.L. and Kumar, S. (1998) Prodomain-dependent nuclear localization of the caspase-2 (Nedd2) precursor. A novel function for a caspase prodomain. $J$ Biol Chem, 273, 24535-24542.

Conti, E. (2002) Structures of importins. Results Probl Cell Differ, 35, 93-113.

Conti, E. and Izaurralde, E. (2001) Nucleocytoplasmic transport enters the atomic age. Curr Opin Cell Biol, 13, 310-319.

Conti, E. and Kuriyan, J. (2000) Crystallographic analysis of the specific yet versatile recognition of distinct nuclear localization signals by karyopherin alpha. Structure Fold Des, 8, 329-338.

Conti, E., Muller, C.W. and Stewart, M. (2006) Karyopherin flexibility in nucleocytoplasmic transport. Curr Opin Struct Biol, 16, 237-244.

Conti, E., Uy, M., Leighton, L., Blobel, G. and Kuriyan, J. (1998) Crystallographic analysis of the recognition of a nuclear localization signal by the nuclear import factor karyopherin alpha. Cell, 94, 193-204.

Cronshaw, J.M., Krutchinsky, A.N., Zhang, W., Chait, B.T. and Matunis, M.J. (2002) Proteomic analysis of the mammalian nuclear pore complex. $J$ Cell Biol, 158, 915-927.

Cryns, V.L., Bergeron, L., Zhu, H., Li, H. and Yuan, J. (1996) Specific cleavage of alpha-fodrin during Fas- and tumor necrosis factor-induced apoptosis is mediated by an interleukin-1beta-converting enzyme/Ced-3 protease distinct from the poly(ADP-ribose) polymerase protease. J Biol Chem, 271, 31277-31282.

Dingwall, C. and Laskey, R.A. (1991) Nuclear targeting sequences--a consensus? Trends Biochem Sci, 16, 478-481.

Dingwall, C., Robbins, J., Dilworth, S.M., Roberts, B. and Richardson, W.D. (1988) The nucleoplasmin nuclear location sequence is larger and more complex than that of SV-40 large T antigen. $J$ Cell Biol, 107, 841-849.

Donepudi, M., Mac Sweeney, A., Briand, C. and Grutter, M.G. (2003) Insights into the regulatory mechanism for caspase-8 activation. Mol Cell, 11, 543-549.

Durrieu, F., Samejima, K., Fortune, J.M., Kandels-Lewis, S., Osheroff, N. and Earnshaw, W.C. (2000) DNA topoisomerase IIalpha interacts with CAD nuclease and is involved in chromatin condensation during apoptotic execution. Curr Biol, 10, 923-926.

Enari, M., Sakahira, H., Yokoyama, H., Okawa, K., Iwamatsu, A. and Nagata, S. (1998) A caspaseactivated DNase that degrades DNA during apoptosis, and its inhibitor ICAD. Nature, 391, 4350. 
Fagerlund, R., Kinnunen, L., Kohler, M., Julkunen, I. and Melen, K. (2005) NF-\{kappa $\}$ B is transported into the nucleus by importin \{alpha\}3 and importin \{alpha\}4. J Biol Chem, 280, 15942-15951.

Fahrenkrog, B. (2006) The nuclear pore complex, nuclear transport, and apoptosis. Can J Physiol Pharmacol, 84, 279-286.

Fahrenkrog, B. and Aebi, U. (2003) The nuclear pore complex: nucleocytoplasmic transport and beyond. Nat Rev Mol Cell Biol, 4, 757-766.

Fahrenkrog, B., Hurt, E.C., Aebi, U. and Pante, N. (1998) Molecular architecture of the yeast nuclear pore complex: localization of Nsp1p subcomplexes. J Cell Biol, 143, 577-588.

Fahrenkrog, B., Stoffler, D. and Aebi, U. (2001) Nuclear pore complex architecture and functional dynamics. Curr Top Microbiol Immunol, 259, 95-117.

Ferrando-May, E. (2005) Nucleocytoplasmic transport in apoptosis. Cell Death Differ, 12, 1263-1276.

Ferrando-May, E., Cordes, V., Biller-Ckovric, I., Mirkovic, J., Gorlich, D. and Nicotera, P. (2001) Caspases mediate nucleoporin cleavage, but not early redistribution of nuclear transport factors and modulation of nuclear permeability in apoptosis. Cell Death Differ, 8, 495-505.

Finlay, D.R., Newmeyer, D.D., Price, T.M. and Forbes, D.J. (1987) Inhibition of in vitro nuclear transport by a lectin that binds to nuclear pores. $J$ Cell Biol, 104, 189-200.

Fischer, U., Huber, J., Boelens, W.C., Mattaj, I.W. and Luhrmann, R. (1995) The HIV-1 Rev activation domain is a nuclear export signal that accesses an export pathway used by specific cellular RNAs. Cell, 82, 475-483.

Fischer, U., Janicke, R.U. and Schulze-Osthoff, K. (2003) Many cuts to ruin: a comprehensive update of caspase substrates. Cell Death Differ, 10, 76-100.

Fontes, M.R., Teh, T., Jans, D., Brinkworth, R.I. and Kobe, B. (2003) Structural basis for the specificity of bipartite nuclear localization sequence binding by importin-alpha. J Biol Chem, 278, 2798127987.

Fontes, M.R., Teh, T. and Kobe, B. (2000) Structural basis of recognition of monopartite and bipartite nuclear localization sequences by mammalian importin-alpha. J Mol Biol, 297, 1183-1194.

Fornerod, M. and Ohno, M. (2002) Exportin-mediated nuclear export of proteins and ribonucleoproteins. Results Probl Cell Differ, 35, 67-91.

Fornerod, M., Ohno, M., Yoshida, M. and Mattaj, I.W. (1997) CRM1 is an export receptor for leucinerich nuclear export signals. Cell, 90, 1051-1060.

Frey, S., Richter, R.P. and Görlich, D. (2006) FG-rich repeats of nuclear pore proteins form a threedimensional meshwork with hydrogel-like properties. Science, 314, 815-817.

Fribourg, S., Braun, I.C., Izaurralde, E. and Conti, E. (2001) Structural basis for the recognition of a nucleoporin FG repeat by the NTF2-like domain of the TAP/p15 mRNA nuclear export factor. Mol Cell, 8, 645-656.

Fried, H. and Kutay, U. (2003) Nucleocytoplasmic transport: taking an inventory. Cell Mol Life Sci, 60, $1659-1688$. 
Friedrich, B., Quensel, C., Sommer, T., Hartmann, E. and Kohler, M. (2006) NLS and protein context both mediate importin \{alpha\} specificity of nuclear import substrates. Mol Cell Biol.

Fuentes-Prior, P. and Salvesen, G.S. (2004) The protein structures that shape caspase activity, specificity, activation and inhibition. Biochem $J, \mathbf{3 8 4}, 201-232$.

Geng, Y.J., Azuma, T., Tang, J.X., Hartwig, J.H., Muszynski, M., Wu, Q., Libby, P. and Kwiatkowski, D.J. (1998) Caspase-3-induced gelsolin fragmentation contributes to actin cytoskeletal collapse, nucleolysis, and apoptosis of vascular smooth muscle cells exposed to proinflammatory cytokines. Eur J Cell Biol, 77, 294-302.

Goebel, W., Obermeyer, N., Bleicher, N., Kratzmeier, M., Eibl, H.J., Doenecke, D. and Albig, W. (2007) Apoptotic DNA fragmentation is not related to the phosphorylation state of histone H1. Biol Chem, 388, 197-206.

Gohring, F. and Fackelmayer, F.O. (1997) The scaffold/matrix attachment region binding protein hnRNP$\mathrm{U}$ (SAF-A) is directly bound to chromosomal DNA in vivo: a chemical cross-linking study. Biochemistry, 36, 8276-8283.

Görlich, D. (1998) Transport into and out of the cell nucleus. Embo J, 17, 2721-2727.

Görlich, D., Dabrowski, M., Bischoff, F.R., Kutay, U., Bork, P., Hartmann, E., Prehn, S. and Izaurralde, E. (1997) A novel class of RanGTP binding proteins. J Cell Biol, 138, 65-80.

Görlich, D., Henklein, P., Laskey, R.A. and Hartmann, E. (1996a) A 41 amino acid motif in importinalpha confers binding to importin-beta and hence transit into the nucleus. Embo J, 15, 1810-1817.

Görlich, D., Kostka, S., Kraft, R., Dingwall, C., Laskey, R.A., Hartmann, E. and Prehn, S. (1995a) Two different subunits of importin cooperate to recognize nuclear localization signals and bind them to the nuclear envelope. Curr Biol, 5, 383-392.

Görlich, D. and Kutay, U. (1999) Transport between the cell nucleus and the cytoplasm. Annu Rev Cell Dev Biol, 15, 607-660.

Görlich, D., Pante, N., Kutay, U., Aebi, U. and Bischoff, F.R. (1996b) Identification of different roles for RanGDP and RanGTP in nuclear protein import. Embo J, 15, 5584-5594.

Görlich, D., Prehn, S., Laskey, R.A. and Hartmann, E. (1994) Isolation of a protein that is essential for the first step of nuclear protein import. Cell, 79, 767-778.

Görlich, D., Vogel, F., Mills, A.D., Hartmann, E. and Laskey, R.A. (1995b) Distinct functions for the two importin subunits in nuclear protein import. Nature, 377, 246-248.

Green, D.R. and Evan, G.I. (2002) A matter of life and death. Cancer Cell, 1, 19-30.

Gu, J., Dong, R.P., Zhang, C., McLaughlin, D.F., Wu, M.X. and Schlossman, S.F. (1999) Functional interaction of DFF35 and DFF45 with caspase-activated DNA fragmentation nuclease DFF40. $J$ Biol Chem, 274, 20759-20762.

Hacker, G. (2000) The morphology of apoptosis. Cell Tissue Res, 301, 5-17.

Halenbeck, R., MacDonald, H., Roulston, A., Chen, T.T., Conroy, L. and Williams, L.T. (1998) CPAN, a human nuclease regulated by the caspase-sensitive inhibitor DFF45. Curr Biol, 8, 537-540. 
Han, Z., Hendrickson, E.A., Bremner, T.A. and Wyche, J.H. (1997) A sequential two-step mechanism for the production of the mature p17:p12 form of caspase-3 in vitro. $J$ Biol Chem, 272, 13432-13436.

Hanahan, D. and Weinberg, R.A. (2000) The hallmarks of cancer. Cell, 100, 57-70.

Henderson, B.R. and Percipalle, P. (1997) Interactions between HIV Rev and nuclear import and export factors: the Rev nuclear localisation signal mediates specific binding to human importin-beta. $J$ Mol Biol, 274, 693-707.

Hengartner, M.O. (2000) The biochemistry of apoptosis. Nature, 407, 770-776.

Hinshaw, J.E., Carragher, B.O. and Milligan, R.A. (1992) Architecture and design of the nuclear pore complex. Cell, 69, 1133-1141.

Hodel, M.R., Corbett, A.H. and Hodel, A.E. (2001) Dissection of a nuclear localization signal. J Biol Chem, 276, 1317-1325.

Hsu, S.C. and Hung, M.C. (2007) Characterization of a novel tripartite nuclear localization sequence in the EGFR family. $J$ Biol Chem.

Inohara, N., Koseki, T., Chen, S., Benedict, M.A. and Nunez, G. (1999) Identification of regulatory and catalytic domains in the apoptosis nuclease DFF40/CAD. J Biol Chem, 274, 270-274.

Izaurralde, E., Kutay, U., von Kobbe, C., Mattaj, I.W. and Görlich, D. (1997) The asymmetric distribution of the constituents of the Ran system is essential for transport into and out of the nucleus. Embo $J, \mathbf{1 6}, 6535-6547$.

Jacobson, M.D., Weil, M. and Raff, M.C. (1997) Programmed cell death in animal development. Cell, 88, 347-354.

Jakel, S. and Görlich, D. (1998) Importin beta, transportin, RanBP5 and RanBP7 mediate nuclear import of ribosomal proteins in mammalian cells. Embo J, 17, 4491-4502.

Jans, D.A., Xiao, C.Y. and Lam, M.H. (2000) Nuclear targeting signal recognition: a key control point in nuclear transport? Bioessays, 22, 532-544.

Kahle, J., Baake, M., Doenecke, D. and Albig, W. (2005) Subunits of the heterotrimeric transcription factor NF-Y are imported into the nucleus by distinct pathways involving importin beta and importin 13. Mol Cell Biol, 25, 5339-5354.

Kalderon, D., Richardson, W.D., Markham, A.F. and Smith, A.E. (1984a) Sequence requirements for nuclear location of simian virus 40 large-T antigen. Nature, 311, 33-38.

Kalderon, D., Roberts, B.L., Richardson, W.D. and Smith, A.E. (1984b) A short amino acid sequence able to specify nuclear location. Cell, 39, 499-509.

Kamada, S., Kikkawa, U., Tsujimoto, Y. and Hunter, T. (2005a) A-kinase-anchoring protein 95 functions as a potential carrier for the nuclear translocation of active caspase 3 through an enzymesubstrate-like association. Mol Cell Biol, 25, 9469-9477.

Kamada, S., Kikkawa, U., Tsujimoto, Y. and Hunter, T. (2005b) Nuclear translocation of caspase-3 is dependent on its proteolytic activation and recognition of a substrate-like protein(s). J Biol Chem, 280, $857-860$. 
Kawane, K., Fukuyama, H., Kondoh, G., Takeda, J., Ohsawa, Y., Uchiyama, Y. and Nagata, S. (2001) Requirement of DNase II for definitive erythropoiesis in the mouse fetal liver. Science, 292, 1546-1549.

Kent, H.M., Clarkson, W.D., Bullock, T.L. and Stewart, M. (1996) Crystallization and preliminary X-ray diffraction analysis of nuclear transport factor 2. J Struct Biol, 116, 326-329.

Kerr, J.F., Wyllie, A.H. and Currie, A.R. (1972) Apoptosis: a basic biological phenomenon with wideranging implications in tissue kinetics. Br J Cancer, 26, 239-257.

Klebe, C., Prinz, H., Wittinghofer, A. and Goody, R.S. (1995) The kinetic mechanism of Ran--nucleotide exchange catalyzed by RCC1. Biochemistry, 34, 12543-12552.

Kobe, B. (1999) Autoinhibition by an internal nuclear localization signal revealed by the crystal structure of mammalian importin alpha. Nat Struct Biol, 6, 388-397.

Kodiha, M., Chu, A., Matusiewicz, N. and Stochaj, U. (2004) Multiple mechanisms promote the inhibition of classical nuclear import upon exposure to severe oxidative stress. Cell Death Differ, 11, 862-874.

Kohler, M., Ansieau, S., Prehn, S., Leutz, A., Haller, H. and Hartmann, E. (1997) Cloning of two novel human importin-alpha subunits and analysis of the expression pattern of the importin-alpha protein family. FEBS Lett, 417, 104-108.

Kohler, M., Speck, C., Christiansen, M., Bischoff, F.R., Prehn, S., Haller, H., Görlich, D. and Hartmann, E. (1999) Evidence for distinct substrate specificities of importin alpha family members in nuclear protein import. Mol Cell Biol, 19, 7782-7791.

Korn, C., Scholz, S.R., Gimadutdinow, O., Lurz, R., Pingoud, A. and Meiss, G. (2005) Interaction of DNA fragmentation factor (DFF) with DNA reveals an unprecedented mechanism for nuclease inhibition and suggests that DFF can be activated in a DNA-bound state. $J$ Biol Chem, 280, 60056015.

Korn, C., Scholz, S.R., Gimadutdinow, O., Pingoud, A. and Meiss, G. (2002) Involvement of conserved histidine, lysine and tyrosine residues in the mechanism of DNA cleavage by the caspase-3 activated DNase CAD. Nucleic Acids Res, 30, 1325-1332.

Kothakota, S., Azuma, T., Reinhard, C., Klippel, A., Tang, J., Chu, K., McGarry, T.J., Kirschner, M.W., Koths, K., Kwiatkowski, D.J. and Williams, L.T. (1997) Caspase-3-generated fragment of gelsolin: effector of morphological change in apoptosis. Science, 278, 294-298.

Krieser, R.J., MacLea, K.S., Longnecker, D.S., Fields, J.L., Fiering, S. and Eastman, A. (2002) Deoxyribonuclease IIalpha is required during the phagocytic phase of apoptosis and its loss causes perinatal lethality. Cell Death Differ, 9, 956-962.

Kutay, U., Bischoff, F.R., Kostka, S., Kraft, R. and Görlich, D. (1997a) Export of importin alpha from the nucleus is mediated by a specific nuclear transport factor. Cell, 90, 1061-1071.

Kutay, U. and Guttinger, S. (2005) Leucine-rich nuclear-export signals: born to be weak. Trends Cell Biol, 15, 121-124. 
Kutay, U., Izaurralde, E., Bischoff, F.R., Mattaj, I.W. and Görlich, D. (1997b) Dominant-negative mutants of importin-beta block multiple pathways of import and export through the nuclear pore complex. Embo J, 16, 1153-1163.

Laemmli, U.K. (1970) Cleavage of structural proteins during the assembly of the head of bacteriophage T4. Nature, 227, 680-685.

Lagarkova, M.A., Iarovaia, O.V. and Razin, S.V. (1995) Large-scale fragmentation of mammalian DNA in the course of apoptosis proceeds via excision of chromosomal DNA loops and their oligomers. J Biol Chem, 270, 20239-20241.

Lechardeur, D., Dougaparsad, S., Nemes, C. and Lukacs, G.L. (2005) Oligomerization state of the DNA fragmentation factor in normal and apoptotic cells. J Biol Chem, 280, 40216-40225.

Lechardeur, D., Drzymala, L., Sharma, M., Zylka, D., Kinach, R., Pacia, J., Hicks, C., Usmani, N., Rommens, J.M. and Lukacs, G.L. (2000) Determinants of the nuclear localization of the heterodimeric DNA fragmentation factor (ICAD/CAD). J Cell Biol, 150, 321-334.

Lee, J.W., Soung, Y.H., Kim, S.Y., Park, W.S., Nam, S.W., Lee, J.Y., Yoo, N.J. and Lee, S.H. (2006) Somatic mutation of pro-apoptosis caspase- 6 gene is rare in breast and lung carcinomas. Pathology, 38, 358-359.

Lee, S.J., Imamoto, N., Sakai, H., Nakagawa, A., Kose, S., Koike, M., Yamamoto, M., Kumasaka, T., Yoneda, Y. and Tsukihara, T. (2000) The adoption of a twisted structure of importin-beta is essential for the protein-protein interaction required for nuclear transport. $J$ Mol Biol, 302, 251264.

Leung, S.W., Harreman, M.T., Hodel, M.R., Hodel, A.E. and Corbett, A.H. (2003) Dissection of the karyopherin alpha nuclear localization signal (NLS)-binding groove: functional requirements for NLS binding. $J$ Biol Chem, 278, 41947-41953.

Li, L.Y., Luo, X. and Wang, X. (2001) Endonuclease G is an apoptotic DNase when released from mitochondria. Nature, 412, 95-99.

Liku, M.E., Nguyen, V.Q., Rosales, A.W., Irie, K. and Li, J.J. (2005) CDK phosphorylation of a novel NLS-NES module distributed between two subunits of the Mcm2-7 complex prevents chromosomal rereplication. Mol Biol Cell, 16, 5026-5039.

Liu, X., Li, P., Widlak, P., Zou, H., Luo, X., Garrard, W.T. and Wang, X. (1998) The 40-kDa subunit of DNA fragmentation factor induces DNA fragmentation and chromatin condensation during apoptosis. Proc Natl Acad Sci U S A, 95, 8461-8466.

Liu, X., Zou, H., Slaughter, C. and Wang, X. (1997) DFF, a heterodimeric protein that functions downstream of caspase-3 to trigger DNA fragmentation during apoptosis. Cell, 89, 175-184.

Liu, X., Zou, H., Widlak, P., Garrard, W. and Wang, X. (1999) Activation of the apoptotic endonuclease DFF40 (caspase-activated DNase or nuclease). Oligomerization and direct interaction with histone H1. J Biol Chem, 274, 13836-13840. 
Luo, J.C. and Shibuya, M. (2001) A variant of nuclear localization signal of bipartite-type is required for the nuclear translocation of hypoxia inducible factors (1alpha, 2alpha and 3alpha). Oncogene, 20, $1435-1444$.

Macara, I.G. (2001) Transport into and out of the nucleus. Microbiol Mol Biol Rev, 65, 570-594, table of contents.

Madrid, A.S. and Weis, K. (2006) Nuclear transport is becoming crystal clear. Chromosoma, 115, 98-109.

Mahajan, R., Delphin, C., Guan, T., Gerace, L. and Melchior, F. (1997) A small ubiquitin-related polypeptide involved in targeting RanGAP1 to nuclear pore complex protein RanBP2. Cell, 88, 97-107.

Makkerh, J.P., Dingwall, C. and Laskey, R.A. (1996) Comparative mutagenesis of nuclear localization signals reveals the importance of neutral and acidic amino acids. Curr Biol, 6, 1025-1027.

Malik, H.S., Eickbush, T.H. and Goldfarb, D.S. (1997) Evolutionary specialization of the nuclear targeting apparatus. Proc Natl Acad Sci U S A, 94, 13738-13742.

Mandal, M., Adam, L. and Kumar, R. (1999) Redistribution of activated caspase-3 to the nucleus during butyric acid-induced apoptosis. Biochem Biophys Res Commun, 260, 775-780.

Martin, S.J., Newmeyer, D.D., Mathias, S., Farschon, D.M., Wang, H.G., Reed, J.C., Kolesnick, R.N. and Green, D.R. (1995) Cell-free reconstitution of Fas-, UV radiation- and ceramide-induced apoptosis. Embo J, 14, 5191-5200.

Mashima, T., Naito, M. and Tsuruo, T. (1999) Caspase-mediated cleavage of cytoskeletal actin plays a positive role in the process of morphological apoptosis. Oncogene, 18, 2423-2430.

Matsuura, Y. and Stewart, M. (2004) Structural basis for the assembly of a nuclear export complex. Nature, 432, 872-877.

Mattaj, I.W. and Englmeier, L. (1998) Nucleocytoplasmic transport: the soluble phase. Annu Rev Biochem, 67, 265-306.

Matunis, M.J., Coutavas, E. and Blobel, G. (1996) A novel ubiquitin-like modification modulates the partitioning of the Ran-GTPase-activating protein RanGAP1 between the cytosol and the nuclear pore complex. J Cell Biol, 135, 1457-1470.

McIlroy, D., Tanaka, M., Sakahira, H., Fukuyama, H., Suzuki, M., Yamamura, K., Ohsawa, Y., Uchiyama, Y. and Nagata, S. (2000) An auxiliary mode of apoptotic DNA fragmentation provided by phagocytes. Genes Dev, 14, 549-558.

Meiss, G., Scholz, S.R., Korn, C., Gimadutdinow, O. and Pingoud, A. (2001) Identification of functionally relevant histidine residues in the apoptotic nuclease CAD. Nucleic Acids Res, 29, 3901-3909.

Melen, K., Fagerlund, R., Franke, J., Kohler, M., Kinnunen, L. and Julkunen, I. (2003) Importin alpha nuclear localization signal binding sites for STAT1, STAT2, and influenza A virus nucleoprotein. J Biol Chem, 278, 28193-28200. 
Mingot, J.M., Bohnsack, M.T., Jakle, U. and Görlich, D. (2004) Exportin 7 defines a novel general nuclear export pathway. Embo J, 23, 3227-3236.

Mingot, J.M., Kostka, S., Kraft, R., Hartmann, E. and Görlich, D. (2001) Importin 13: a novel mediator of nuclear import and export. Embo $J, 20,3685-3694$.

Miyamoto, Y., Imamoto, N., Sekimoto, T., Tachibana, T., Seki, T., Tada, S., Enomoto, T. and Yoneda, Y. (1997) Differential modes of nuclear localization signal (NLS) recognition by three distinct classes of NLS receptors. $J$ Biol Chem, 272, 26375-26381.

Miyamoto, Y., Saiwaki, T., Yamashita, J., Yasuda, Y., Kotera, I., Shibata, S., Shigeta, M., Hiraoka, Y., Haraguchi, T. and Yoneda, Y. (2004) Cellular stresses induce the nuclear accumulation of importin alpha and cause a conventional nuclear import block. J Cell Biol, 165, 617-623.

Mosammaparast, N., Guo, Y., Shabanowitz, J., Hunt, D.F. and Pemberton, L.F. (2002) Pathways mediating the nuclear import of histones $\mathrm{H} 3$ and $\mathrm{H} 4$ in yeast. $J$ Biol Chem, 277, 862-868.

Mosammaparast, N., Jackson, K.R., Guo, Y., Brame, C.J., Shabanowitz, J., Hunt, D.F. and Pemberton, L.F. (2001) Nuclear import of histone H2A and H2B is mediated by a network of karyopherins. $J$ Cell Biol, 153, 251-262.

Muhlhausser, P., Muller, E.C., Otto, A. and Kutay, U. (2001) Multiple pathways contribute to nuclear import of core histones. EMBO Rep, 2, 690-696.

Muzio, M., Chinnaiyan, A.M., Kischkel, F.C., O'Rourke, K., Shevchenko, A., Ni, J., Scaffidi, C., Bretz, J.D., Zhang, M., Gentz, R., Mann, M., Krammer, P.H., Peter, M.E. and Dixit, V.M. (1996) FLICE, a novel FADD-homologous ICE/CED-3-like protease, is recruited to the CD95 (Fas/APO-1) death--inducing signaling complex. Cell, 85, 817-827.

Muzio, M., Stockwell, B.R., Stennicke, H.R., Salvesen, G.S. and Dixit, V.M. (1998) An induced proximity model for caspase-8 activation. J Biol Chem, 273, 2926-2930.

Nagata, S., Nagase, H., Kawane, K., Mukae, N. and Fukuyama, H. (2003) Degradation of chromosomal DNA during apoptosis. Cell Death Differ, 10, 108-116.

Oberhammer, F., Wilson, J.W., Dive, C., Morris, I.D., Hickman, J.A., Wakeling, A.E., Walker, P.R. and Sikorska, M. (1993) Apoptotic death in epithelial cells: cleavage of DNA to 300 and/or $50 \mathrm{~kb}$ fragments prior to or in the absence of internucleosomal fragmentation. Embo J, 12, 3679-3684.

Odaka, C. and Mizuochi, T. (2002) Macrophages are involved in DNA degradation of apoptotic cells in murine thymus after administration of hydrocortisone. Cell Death Differ, 9, 104-112.

Otomo, T., Sakahira, H., Uegaki, K., Nagata, S. and Yamazaki, T. (2000) Structure of the heterodimeric complex between CAD domains of CAD and ICAD. Nat Struct Biol, 7, 658-662.

Pante, N. and Kann, M. (2002) Nuclear pore complex is able to transport macromolecules with diameters of about 39 nm. Mol Biol Cell, 13, 425-434.

Paraskeva, E., Izaurralde, E., Bischoff, F.R., Huber, J., Kutay, U., Hartmann, E., Luhrmann, R. and Görlich, D. (1999) CRM1-mediated recycling of snurportin 1 to the cytoplasm. J Cell Biol, 145, $255-264$ 
Patre, M., Tabbert, A., Hermann, D., Walczak, H., Rackwitz, H.R., Cordes, V.C. and Ferrando-May, E. (2006) Caspases target only two architectural components within the core structure of the nuclear pore complex. J Biol Chem, 281, 1296-1304.

Peifer, M., Berg, S. and Reynolds, A.B. (1994) A repeating amino acid motif shared by proteins with diverse cellular roles. Cell, 76, 789-791.

Pemberton, L.F. and Paschal, B.M. (2005) Mechanisms of receptor-mediated nuclear import and nuclear export. Traffic, 6, 187-198.

Pemberton, L.F., Rosenblum, J.S. and Blobel, G. (1999) Nuclear import of the TATA-binding protein: mediation by the karyopherin Kap114p and a possible mechanism for intranuclear targeting. $J$ Cell Biol, 145, 1407-1417.

Petosa, C., Schoehn, G., Askjaer, P., Bauer, U., Moulin, M., Steuerwald, U., Soler-Lopez, M., Baudin, F., Mattaj, I.W. and Muller, C.W. (2004) Architecture of CRM1/Exportin1 suggests how cooperativity is achieved during formation of a nuclear export complex. Mol Cell, 16, 761-775.

Pollard, V.W., Michael, W.M., Nakielny, S., Siomi, M.C., Wang, F. and Dreyfuss, G. (1996) A novel receptor-mediated nuclear protein import pathway. Cell, 86, 985-994.

Quensel, C., Friedrich, B., Sommer, T., Hartmann, E. and Kohler, M. (2004) In vivo analysis of importin alpha proteins reveals cellular proliferation inhibition and substrate specificity. Mol Cell Biol, 24, 10246-10255.

Rao, L., Perez, D. and White, E. (1996) Lamin proteolysis facilitates nuclear events during apoptosis. $J$ Cell Biol, 135, 1441-1455.

Rathmell, J.C. and Thompson, C.B. (2002) Pathways of apoptosis in lymphocyte development, homeostasis, and disease. Cell, 109 Suppl, S97-107.

Rexach, M. and Blobel, G. (1995) Protein import into nuclei: association and dissociation reactions involving transport substrate, transport factors, and nucleoporins. Cell, 83, 683-692.

Ribbeck, K. and Görlich, D. (2001) Kinetic analysis of translocation through nuclear pore complexes. Embo J, 20, 1320-1330.

Ribbeck, K. and Görlich, D. (2002) The permeability barrier of nuclear pore complexes appears to operate via hydrophobic exclusion. Embo J, 21, 2664-2671.

Ribbeck, K., Lipowsky, G., Kent, H.M., Stewart, M. and Görlich, D. (1998) NTF2 mediates nuclear import of Ran. Embo J, 17, 6587-6598.

Rich, T., Allen, R.L. and Wyllie, A.H. (2000) Defying death after DNA damage. Nature, 407, 777-783.

Riedl, S.J., Fuentes-Prior, P., Renatus, M., Kairies, N., Krapp, S., Huber, R., Salvesen, G.S. and Bode, W. (2001) Structural basis for the activation of human procaspase-7. Proc Natl Acad Sci U S A, 98 , 14790-14795.

Robbins, J., Dilworth, S.M., Laskey, R.A. and Dingwall, C. (1991) Two interdependent basic domains in nucleoplasmin nuclear targeting sequence: identification of a class of bipartite nuclear targeting sequence. Cell, 64, 615-623. 
Rodriguez, M., Benito, A., Tubert, P., Castro, J., Ribo, M., Beaumelle, B. and Vilanova, M. (2006) A cytotoxic ribonuclease variant with a discontinuous nuclear localization signal constituted by basic residues scattered over three areas of the molecule. J Mol Biol, 360, 548-557.

Romanelli, M.G. and Morandi, C. (2002) Importin alpha binds to an unusual bipartite nuclear localization signal in the heterogeneous ribonucleoprotein type I. Eur J Biochem, 269, 2727-2734.

Rout, M.P. and Aitchison, J.D. (2001) The nuclear pore complex as a transport machine. J Biol Chem, 276, 16593-16596.

Rout, M.P., Aitchison, J.D., Suprapto, A., Hjertaas, K., Zhao, Y. and Chait, B.T. (2000) The yeast nuclear pore complex: composition, architecture, and transport mechanism. J Cell Biol, 148, 635-651.

Rout, M.P. and Wente, S.R. (1994) Pores for thought: nuclear pore complex proteins. Trends Cell Biol, 4, 357-365.

Ruiz-Carrillo, A. and Renaud, J. (1987) Endonuclease G: a (dG)n X (dC)n-specific DNase from higher eukaryotes. Embo J, 6, 401-407.

Sakahira, H., Enari, M. and Nagata, S. (1998) Cleavage of CAD inhibitor in CAD activation and DNA degradation during apoptosis. Nature, 391, 96-99.

Sakahira, H., Enari, M. and Nagata, S. (1999) Functional differences of two forms of the inhibitor of caspase-activated DNase, ICAD-L, and ICAD-S. J Biol Chem, 274, 15740-15744.

Sakahira, H., Iwamatsu, A. and Nagata, S. (2000) Specific chaperone-like activity of inhibitor of caspaseactivated DNase for caspase-activated DNase. J Biol Chem, 275, 8091-8096.

Sakahira, H. and Nagata, S. (2002) Co-translational folding of caspase-activated DNase with Hsp70, Hsp40, and inhibitor of caspase-activated DNase. J Biol Chem, 277, 3364-3370.

Sakahira, H., Takemura, Y. and Nagata, S. (2001) Enzymatic active site of caspase-activated DNase (CAD) and its inhibition by inhibitor of CAD. Arch Biochem Biophys, 388, 91-99.

Salvesen, G.S. and Dixit, V.M. (1997) Caspases: intracellular signaling by proteolysis. Cell, 91, 443-446.

Salvesen, G.S. and Dixit, V.M. (1999) Caspase activation: the induced-proximity model. Proc Natl Acad Sci U S A, 96, 10964-10967.

Samejima, K. and Earnshaw, W.C. (1998) ICAD/DFF regulator of apoptotic nuclease is nuclear. Exp Cell Res, 243, 453-459.

Samejima, K. and Earnshaw, W.C. (2000) Differential localization of ICAD-L and ICAD-S in cells due to removal of a C-terminal NLS from ICAD-L by alternative splicing. Exp Cell Res, 255, 314320.

Samejima, K., Tone, S. and Earnshaw, W.C. (2001) CAD/DFF40 nuclease is dispensable for high molecular weight DNA cleavage and stage I chromatin condensation in apoptosis. $J$ Biol Chem, 276, 45427-45432.

Sanger, F., Nicklen, S. and Coulson, A.R. (1977) DNA sequencing with chain-terminating inhibitors. Proc Natl Acad Sci U S A, 74, 5463-5467.

Savill, J. and Fadok, V. (2000) Corpse clearance defines the meaning of cell death. Nature, 407, 784-788. 
Sekimoto, T., Imamoto, N., Nakajima, K., Hirano, T. and Yoneda, Y. (1997) Extracellular signaldependent nuclear import of Stat1 is mediated by nuclear pore-targeting complex formation with NPI-1, but not Rch1. Embo J, 16, 7067-7077.

Sharif-Askari, E., Alam, A., Rheaume, E., Beresford, P.J., Scotto, C., Sharma, K., Lee, D., DeWolf, W.E., Nuttall, M.E., Lieberman, J. and Sekaly, R.P. (2001) Direct cleavage of the human DNA fragmentation factor-45 by granzyme B induces caspase-activated DNase release and DNA fragmentation. Embo J, 20, 3101-3113.

Sharp, P.A., Sugden, B. and Sambrook, J. (1973) Detection of two restriction endonuclease activities in Haemophilus parainfluenzae using analytical agarose--ethidium bromide electrophoresis. Biochemistry, 12, 3055-3063.

Shi, W.Y. and Skeath, J.B. (2004) The Drosophila RCC1 homolog, Bj1, regulates nucleocytoplasmic transport and neural differentiation during Drosophila development. Dev Biol, 270, 106-121.

Shikama, Y., U, M., Miyashita, T. and Yamada, M. (2001) Comprehensive studies on subcellular localizations and cell death-inducing activities of eight GFP-tagged apoptosis-related caspases. Exp Cell Res, 264, 315-325.

Shim, S.R., Kook, S., Kim, J.I. and Song, W.K. (2001) Degradation of focal adhesion proteins paxillin and p130cas by caspases or calpains in apoptotic rat-1 and L929 cells. Biochem Biophys Res Commun, 286, 601-608.

Smith, A., Brownawell, A. and Macara, I.G. (1998) Nuclear import of Ran is mediated by the transport factor NTF2. Curr Biol, 8, 1403-1406.

Stade, K., Ford, C.S., Guthrie, C. and Weis, K. (1997) Exportin 1 (Crm1p) is an essential nuclear export factor. Cell, 90, 1041-1050.

Stewart, M. (2003) Structural biology. Nuclear trafficking. Science, 302, 1513-1514.

Stoffler, D., Feja, B., Fahrenkrog, B., Walz, J., Typke, D. and Aebi, U. (2003) Cryo-electron tomography provides novel insights into nuclear pore architecture: implications for nucleocytoplasmic transport. J Mol Biol, 328, 119-130.

Strom, A.C. and Weis, K. (2001) Importin-beta-like nuclear transport receptors. Genome Biol, 2, reviews 3008.

Stuven, T., Hartmann, E. and Görlich, D. (2003) Exportin 6: a novel nuclear export receptor that is specific for profilin.actin complexes. Embo J, 22, 5928-5940.

Susin, S.A., Lorenzo, H.K., Zamzami, N., Marzo, I., Snow, B.E., Brothers, G.M., Mangion, J., Jacotot, E., Costantini, P., Loeffler, M., Larochette, N., Goodlett, D.R., Aebersold, R., Siderovski, D.P., Penninger, J.M. and Kroemer, G. (1999) Molecular characterization of mitochondrial apoptosisinducing factor. Nature, 397, 441-446.

Thomas, D.A., Du, C., Xu, M., Wang, X. and Ley, T.J. (2000) DFF45/ICAD can be directly processed by granzyme B during the induction of apoptosis. Immunity, 12, 621-632.

Thompson, C.B. (1995) Apoptosis in the pathogenesis and treatment of disease. Science, 267, 1456-1462. 
Thornberry, N.A., Rano, T.A., Peterson, E.P., Rasper, D.M., Timkey, T., Garcia-Calvo, M., Houtzager, V.M., Nordstrom, P.A., Roy, S., Vaillancourt, J.P., Chapman, K.T. and Nicholson, D.W. (1997) A combinatorial approach defines specificities of members of the caspase family and granzyme B. Functional relationships established for key mediators of apoptosis. J Biol Chem, 272, 1790717911.

Truant, R. and Cullen, B.R. (1999) The arginine-rich domains present in human immunodeficiency virus type 1 Tat and Rev function as direct importin beta-dependent nuclear localization signals. Mol Cell Biol, 19, 1210-1217.

van Loo, G., Schotte, P., van Gurp, M., Demol, H., Hoorelbeke, B., Gevaert, K., Rodriguez, I., RuizCarrillo, A., Vandekerckhove, J., Declercq, W., Beyaert, R. and Vandenabeele, P. (2001) Endonuclease G: a mitochondrial protein released in apoptosis and involved in caspaseindependent DNA degradation. Cell Death Differ, 8, 1136-1142.

Vanags, D.M., Porn-Ares, M.I., Coppola, S., Burgess, D.H. and Orrenius, S. (1996) Protease involvement in fodrin cleavage and phosphatidylserine exposure in apoptosis. J Biol Chem, 271, 31075-31085.

Vaux, D.L. and Flavell, R.A. (2000) Apoptosis genes and autoimmunity. Curr Opin Immunol, 12, 719724.

Vetter, I.R., Arndt, A., Kutay, U., Görlich, D. and Wittinghofer, A. (1999) Structural view of the RanImportin beta interaction at 2.3 A resolution. Cell, 97, 635-646.

Vincenz, C. and Dixit, V.M. (1997) Fas-associated death domain protein interleukin-1 beta-converting enzyme 2 (FLICE2), an ICE/Ced-3 homologue, is proximally involved in CD95- and p55mediated death signaling. J Biol Chem, 272, 6578-6583.

Weber, K. and Osborn, M. (1969) The reliability of molecular weight determinations by dodecyl sulfatepolyacrylamide gel electrophoresis. J Biol Chem, 244, 4406-4412.

Wei, Y., Fox, T., Chambers, S.P., Sintchak, J., Coll, J.T., Golec, J.M., Swenson, L., Wilson, K.P. and Charifson, P.S. (2000) The structures of caspases-1, $-3,-7$ and -8 reveal the basis for substrate and inhibitor selectivity. Chem Biol, 7, 423-432.

Weis, K. (2003) Regulating access to the genome: nucleocytoplasmic transport throughout the cell cycle. Cell, 112, 441-451.

Weis, K., Dingwall, C. and Lamond, A.I. (1996a) Characterization of the nuclear protein import mechanism using Ran mutants with altered nucleotide binding specificities. Embo J, 15, 71207128.

Weis, K., Ryder, U. and Lamond, A.I. (1996b) The conserved amino-terminal domain of hSRP1 alpha is essential for nuclear protein import. Embo J, 15, 1818-1825.

Wen, W., Meinkoth, J.L., Tsien, R.Y. and Taylor, S.S. (1995) Identification of a signal for rapid export of proteins from the nucleus. Cell, 82, 463-473. 
Whitehurst, A.W., Wilsbacher, J.L., You, Y., Luby-Phelps, K., Moore, M.S. and Cobb, M.H. (2002) ERK2 enters the nucleus by a carrier-independent mechanism. Proc Natl Acad Sci U S A, 99, 7496-7501.

Widlak, P. and Garrard, W.T. (2005) Discovery, regulation, and action of the major apoptotic nucleases DFF40/CAD and endonuclease G. J Cell Biochem, 94, 1078-1087.

Widlak, P., Kalinowska, M., Parseghian, M.H., Lu, X., Hansen, J.C. and Garrard, W.T. (2005) The histone H1 C-terminal domain binds to the apoptotic nuclease, DNA fragmentation factor (DFF40/CAD) and stimulates DNA cleavage. Biochemistry, 44, 7871-7878.

Widlak, P., Lanuszewska, J., Cary, R.B. and Garrard, W.T. (2003) Subunit structures and stoichiometries of human DNA fragmentation factor proteins before and after induction of apoptosis. $J$ Biol Chem, 278, 26915-26922.

Widlak, P., Li, L.Y., Wang, X. and Garrard, W.T. (2001) Action of recombinant human apoptotic endonuclease $\mathrm{G}$ on naked DNA and chromatin substrates: cooperation with exonuclease and DNase I. J Biol Chem, 276, 48404-48409.

Widlak, P., Li, P., Wang, X. and Garrard, W.T. (2000) Cleavage preferences of the apoptotic endonuclease DFF40 (caspase-activated DNase or nuclease) on naked DNA and chromatin substrates. J Biol Chem, 275, 8226-8232.

Woo, E.J., Kim, Y.G., Kim, M.S., Han, W.D., Shin, S., Robinson, H., Park, S.Y. and Oh, B.H. (2004) Structural mechanism for inactivation and activation of CAD/DFF40 in the apoptotic pathway. Mol Cell, 14, 531-539.

Wyllie, A.H. (1980) Glucocorticoid-induced thymocyte apoptosis is associated with endogenous endonuclease activation. Nature, 284, 555-556.

Yakovlev, A.G., Di, X., Movsesyan, V., Mullins, P.G., Wang, G., Boulares, H., Zhang, J., Xu, M. and Faden, A.I. (2001) Presence of DNA fragmentation and lack of neuroprotective effect in DFF45 knockout mice subjected to traumatic brain injury. Mol Med, 7, 205-216.

Yan, B., Wang, H., Peng, Y., Hu, Y., Zhang, X., Chen, Q., Bedford, J.S., Dewhirst, M.W. and Li, C.Y. (2006a) A unique role of the DNA fragmentation factor in maintaining genomic stability. Proc Natl Acad Sci U S A, 103, 1504-1509.

Yan, B., Wang, H., Zhuo, D., Li, F., Kon, T., Dewhirst, M. and Li, C.Y. (2006b) Apoptotic DNA fragmentation factor maintains chromosome stability in a P53-independent manner. Oncogene, 25, 5370-5376

Yang, Q., Rout, M.P. and Akey, C.W. (1998) Three-dimensional architecture of the isolated yeast nuclear pore complex: functional and evolutionary implications. Mol Cell, 1, 223-234.

Yasuhara, N., Eguchi, Y., Tachibana, T., Imamoto, N., Yoneda, Y. and Tsujimoto, Y. (1997) Essential role of active nuclear transport in apoptosis. Genes Cells, 2, 55-64. 
Yasuhara, N., Shibazaki, N., Tanaka, S., Nagai, M., Kamikawa, Y., Oe, S., Asally, M., Kamachi, Y., Kondoh, H. and Yoneda, Y. (2007) Triggering neural differentiation of ES cells by subtype switching of importin-alpha. Nat Cell Biol, 9, 72-79.

Yoneda, Y., Imamoto-Sonobe, N., Yamaizumi, M. and Uchida, T. (1987) Reversible inhibition of protein import into the nucleus by wheat germ agglutinin injected into cultured cells. Exp Cell Res, 173, 586-595.

Zhang, J., Lee, H., Lou, D.W., Bovin, G.P. and Xu, M. (2000) Lack of obvious 50 kilobase pair DNA fragments in DNA fragmentation factor 45-deficient thymocytes upon activation of apoptosis. Biochem Biophys Res Commun, 274, 225-229.

Zhang, J., Liu, X., Scherer, D.C., van Kaer, L., Wang, X. and Xu, M. (1998) Resistance to DNA fragmentation and chromatin condensation in mice lacking the DNA fragmentation factor 45. Proc Natl Acad Sci U S A, 95, 12480-12485.

Zhang, J., Wang, X., Bove, K.E. and Xu, M. (1999) DNA fragmentation factor 45-deficient cells are more resistant to apoptosis and exhibit different dying morphology than wild-type control cells. $J$ Biol Chem, 274, 37450-37454.

Zhang, J. and Xu, M. (2002) Apoptotic DNA fragmentation and tissue homeostasis. Trends Cell Biol, 12, 84-89.

Zhivotovsky, B., Samali, A., Gahm, A. and Orrenius, S. (1999) Caspases: their intracellular localization and translocation during apoptosis. Cell Death Differ, 6, 644-651.

Zhivotovsky, B., Wade, D., Nicotera, P. and Orrenius, S. (1994) Role of nucleases in apoptosis. Int Arch Allergy Immunol, 105, 333-338.

Zhou, P., Lugovskoy, A.A., McCarty, J.S., Li, P. and Wagner, G. (2001) Solution structure of DFF40 and DFF45 N-terminal domain complex and mutual chaperone activity of DFF40 and DFF45. Proc Natl Acad Sci U S A, 98, 6051-6055. 


\section{Appendix}

Table 3: List of expression constructs used in this study

\begin{tabular}{|c|c|c|c|c|}
\hline name & protein (aa) & primer / DNA fragment (template) & vector & restriction sites \\
\hline pSN8 & DFF40 (1-338) & OSN3/OSN4 & pET-41a(+) & (XbaI/SpeI)/HindIII \\
\hline pSN27 & DFF45 (1-331) & OSN25/OSN15 & pGEX-4T-1 & EcoRI/XhoI \\
\hline pSN28 & DFF45 (1-331) & OSN16/OSN33 & pET-28b(+) & EcoRI/HindIII \\
\hline pSN29 & DFF45 (1-305) & OSN16/OSN34 & pET-28b $(+)$ & EcoRI/HindIII \\
\hline pSN30 & DFF40 (1-323) & OSN3/OSN28 & pET-41a(+) & (XbaI/SpeI)/HindIII \\
\hline pSN31 & EGFP & OSN30/OSN31 & pET-41a(+) & NdeI/NcoI \\
\hline pSN33 & DFF40 (1-338) & OSN32/OSN4 & pSN31 & SalI/HindIII \\
\hline pSN52 & DFF45 (1-305) & OSN25/OSN57 & pGEX-4T-1 & EcoRI/XhoI \\
\hline pSN55 & GST & OSN60/OSN61 & pEGFP-C1 & BglII/XhoI \\
\hline pSN60 & DFF40 (314-338) & OSN71/OSN72 & pGEX-4T-1 & EcoRI/XhoI \\
\hline pSN61 & DFF45 (296-331) & OSN69/OSN70 & pGEX-4T-1 & EcoRI/XhoI \\
\hline pSN69 & DFF45 (1-305) & OSN67/OSN68 & $\begin{array}{l}\text { pEGFP-EGFP- } \\
\text { GST-C1 }\end{array}$ & HindIII/EcoRI \\
\hline pSN72 & DFF40 (1-338) & OSN12/OSN4 & pET-28b(+) & NdeI/HindIII \\
\hline pSN73 & DFF40 (1-323) & OSN12/OSN28 & pET-28b $(+)$ & NdeI/HindIII \\
\hline pSN74 & DFF45 (1-305) & OSN16/OSN34 & pETDuet-1 & EcoRI/HindIII \\
\hline pSN77 & DFF40 (1-338) & OSN84/OSN85 & pSN55 & HindIII/SalI \\
\hline pSN79 & DFF40 (1-323) & OSN84/OSN86 & pSN55 & HindIII/SalI \\
\hline pSN81 & DFF45 (1-331) & OSN87/OSN88 & pSN55 & HindIII/SalI \\
\hline pSN83 & DFF45 (1-305) & OSN87/OSN89 & pSN55 & HindIII/SalI \\
\hline pSN86 & DFF40 (1-338) & OSN84/OSN85 & pRFP-C1 & HindIII/SalI \\
\hline pSN87 & DFF40 (1-323) & OSN84/OSN86 & pRFP-C1 & HindIII/SalI \\
\hline pSN88 & DFF45 (1-331) & OSN87/OSN88 & pRFP-C1 & HindIII/SalI \\
\hline pSN89 & DFF45 (1-305) & OSN87/OSN89 & pRFP-C1 & HindIII/SalI \\
\hline pSN92 & DFF40RKR335-337AAA & OSN84/OSN91 & pSN55 & HindIII/SalI \\
\hline pSN93 & $\begin{array}{l}\text { DFF40 } \\
\text { RKRK327,329-331AAA }\end{array}$ & OSN84/OSN92 & pSN55 & HindIII/SalI \\
\hline pSN94 & $\begin{array}{l}\text { DFF45 } \\
\text { KRR324,325,327,AAA }\end{array}$ & OSN87/OSN93 & pSN55 & HindIII/SalI \\
\hline pSN97 & DFF45RK307,313AA & OSN94/OSN95 (pSN81) & pSN55 & HindIII/SalI \\
\hline pSN98 & DFF40RKR335-337AAA & OSN84/OSN91 & pRFP-C1 & HindIII/SalI \\
\hline pSN99 & $\begin{array}{l}\text { DFF40 } \\
\text { RKRK327,329-331AAA }\end{array}$ & OSN84/OSN92 & pRFP-C1 & HindIII/SalI \\
\hline pSN100 & $\begin{array}{l}\text { DFF45 } \\
\text { KRR324,325,327,AAA }\end{array}$ & OSN87/OSN93 & pRFP-C1 & HindIII/SalI \\
\hline pSN101 & DFF45RK307,313AA & OSN94/OSN95 (pSN88) & pRFP-C1 & HindIII/SalI \\
\hline pSN103 & Imp $\alpha$ X.1. E388R & OSN98/OSN99 & pQE70 & \\
\hline pSN106 & DFF40 (301-338) & OSN105/OSN106 & $\begin{array}{l}\text { pEGFP-EGFP- } \\
\text { GST-C1 }\end{array}$ & EcoRI/HindIII \\
\hline
\end{tabular}




\begin{tabular}{|c|c|c|c|c|}
\hline pSN107 & DFF40KK308,309AA & OSN107/OSN108 (pSN8) & pET-41a(+) & (XbaI/SpeI)/HindIII \\
\hline pSN108 & DFF40KK308,309AA & OSN107/OSN108 (pSN77) & pSN55 & HindIII/SalI \\
\hline pSN109 & DFF40KK308,309AA & OSN107/OSN108 (pSN86) & pRFP-C1 & HindIII/SalI \\
\hline
\end{tabular}




\section{Danksagung}

Bei Prof. Doenecke möchte ich mich für die Bereitstellung der Doktorarbeit, die wissenschaftliche Unterstützung und die herzliche Aufnahme in seine Arbeitsgruppe bedanken.

Prof. Hardeland danke ich für die freundliche Übernahme des Korreferats.

Werner danke ich für die gute Betreuung und sein fortbestehendes Interesse an meiner Arbeit, auch nachdem er das Forscher-Dasein aufgegeben hatte.

Besonders möchte ich allen Kollegen aus der Arbeitsgruppe Doenecke danken, die dafür gesorgt haben, dass die Doktorarbeit nicht nur Arbeit war. Es hat immer viel Spaß gemacht mit euch! Insbesondere danke ich Christa für ihre tatkräftige Unterstützung im Labor und für schöne Stunden an der frischen Luft oder bei dem einen oder anderen Cocktail. Ein großes Dankeschön gebührt Jörg für die gute Zusammenarbeit, die hilfreichen Diskussionen, die Motivation und alles andere.

Weiterhin danke ich Andreas Nolte und Katja Ditter für die Sequenzierungen, den Mitgliedern des Graduiertenkollegs und allen anderen, die mir während meiner Doktorarbeit geholfen haben.

Schließlich möchte ich mich noch bei den Personen bedanken, die zwar nicht direkt an der Doktorarbeit beteiligt waren, ohne die ein Durchhalten aber nicht möglich gewesen wäre. Steffi danke ich für Zuspruch in Frustrationszeiten und die entspannenden freitäglichen Kaffeestunden. Meinen Eltern danke ich für ihre fortwährende Unterstützung, ihr Verständnis und die Ermutigung. 


\section{Curriculum Vitae}

$\begin{array}{ll}\text { Name } & \text { Neimanis } \\ \text { Vorname } & \text { Sonja } \\ \text { Geburtsdatum } & 25.05 .1979 \\ \text { Geburtsort } & \text { Hannover } \\ \text { Staatsangehörigkeit } & \text { deutsch }\end{array}$

$1985-1989$

Grundschule Langenhagen Krähenwinkel

$1989-1991$

Orientierungsstufe Brinker Schule, Langenhagen

$1991-1998$

Gymnasium Langenhagen

$6 / 1998$

Abschluss: Allgemeine Hochschulreife, Note 2,1

$10 / 1998-6 / 2003$

Studium der Biologie an der TU Braunschweig

$10 / 2000$

Diplomvorprüfung

$8 / 2002$

Diplomprüfung in den Fächern Botanik, Zellbiologie und

Biochemie

$9 / 2002-5 / 2003$

Diplomarbeit am Zoologischen Institut in der Arbeitsgruppe

Zellbiologie unter Anleitung von Prof. Dr. B. M. Jockusch

Thema: Analyse der Serinphosphorylierung des Vinculins durch

Protein Kinase C

$6 / 2003$

Abschluss: Diplom-Biologin, Note ,sehr gut“

$8 / 2003-3 / 2007$

Doktorarbeit am Institut für Biochemie und Molekulare

Zellbiologie der Georg-August-Universität zu Göttingen in der

Abteilung Molekularbiologie unter Anleitung von Prof. Dr. D.

Doenecke

Thema: „Nuclear transport of the DNA fragmentation factor via the classical importin $\alpha / \beta$-pathway" 